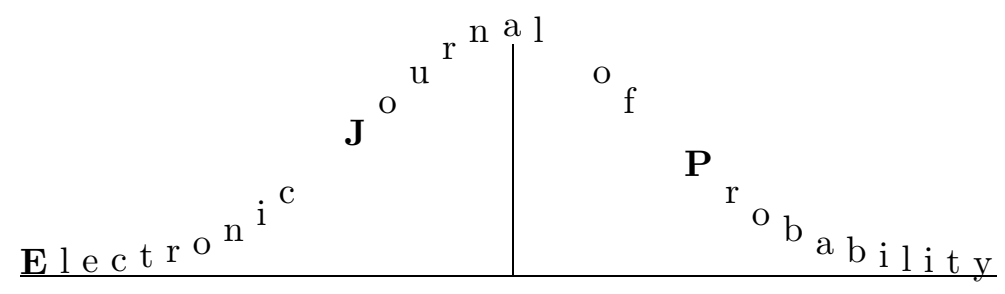

Vol. 9 (2004), Paper no. 15, pages 436-507.

Journal URL

http://www.math.washington.edu/ ejpecp/

\title{
Reconstructing a Multicolor Random Scenery \\ seen along a Random Walk Path with Bounded Jumps
}

\author{
Matthias Löwe \\ Fakultät für Mathematik, Universität Münster \\ Einsteinstraße 62, D-48149 Münster, Germany \\ maloewe@math.uni-muenster.de \\ Heinrich Matzinger \\ Georgia Tech, School of Mathematics \\ Atlanta, GA 30332 0160, USA \\ matzi@math.gatech.edu \\ Franz Merkl \\ Mathematical Institute, Leiden University \\ P.O. Box 9512, 2300 RA Leiden, The Netherlands \\ merkl@math.leidenuniv.nl
}

\begin{abstract}
Kesten [12] noticed that the scenery reconstruction method proposed by Matzinger [18] relies heavily on the skip-free property of the random walk. He asked if one can still reconstruct an i.i.d. scenery seen along the path of a non-skip-free random walk. In this article, we positively answer this question. We prove that if there are enough colors and if the random walk is recurrent with at most bounded jumps, and if it can reach every integer, then one can almost surely reconstruct almost every scenery up to translations and reflections. Our reconstruction method works if there are more colors in the scenery than possible single steps for the random walk.
\end{abstract}

Keywords: Scenery reconstruction, jumps, stationary processes, random walk, ergodic theory

AMS subject classification (2000): Primary 60K37, Secondary 60G10, 60J75

Submitted to EJP on July 3, 2002. Final version accepted on May 15, 2004. 


\section{Contents}

1 Introduction and Result $\quad 438$

2 Some notation $\quad 441$

2.1 Conventions concerning constants . . . . . . . . . . . . . . . . . 442

3 Skeleton of the Reconstruction Procedure 443

4 Heart of the Reconstruction Procedure:

$\begin{array}{ll}\text { Definition of the Algorithm } \mathrm{Alg}^{n} & 448\end{array}$

5 Proofs concerning the Skeleton Structure $\quad 450$

6 Playing Puzzle: Correctness of the Algorithm $\mathrm{Alg}^{n} \quad 456$

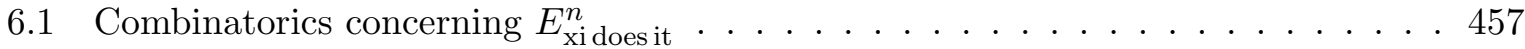

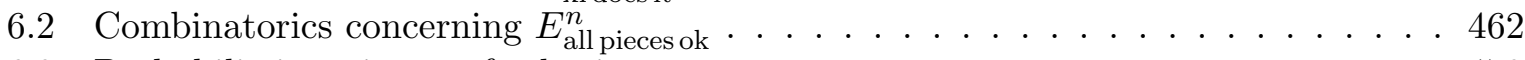

6.3 Probabilistic estimates for basic events . . . . . . . . . . . . . . . . . 473

7 How to find back:

Correctness of the stopping times $T_{f} \quad 480$

8 Getting started: The first stopping times 483

8.1 Properties of the modified measure $\tilde{P} \ldots \ldots \ldots$. . . . . . . . . . . . . 483

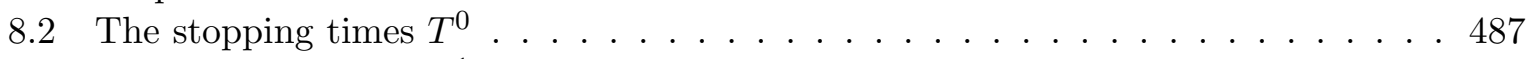

8.3 The stopping times $T^{1} \ldots \ldots \ldots \ldots \ldots \ldots \ldots$ 


\section{Introduction and Result}

A (one dimensional) scenery is a coloring $\xi$ of the integers $\mathbb{Z}$ with $C_{0}$ colors $\left\{1, \ldots, C_{0}\right\}$. Two sceneries $\xi, \xi^{\prime}$ are called equivalent, $\xi \approx \xi^{\prime}$, if one of them is obtained from the other by a translation or reflection. Let $(S(t))_{t \geq 0}$ be a recurrent random walk on the integers. Observing the scenery $\xi$ along the path of this random walk, one sees the color $\xi(S(t))$ at time $t$. The scenery reconstruction problem is concerned with trying to retrieve the scenery $\xi$, given only the sequence of observations $\chi:=(\xi(S(t)))_{t \geq 0}$. Quite obviously retrieving a scenery can only work up to equivalence. Work on the scenery reconstruction problem started by Kesten's question, whether one can recognize a single defect in a random scenery. Kesten [11] answered this question in the affirmative in the case of four colors. He takes the colors to be i.i.d. uniformly distributed. In his Ph.D. thesis [18], see also [17] and [19], Matzinger has proved that typical sceneries can be reconstructed: He takes the sceneries as independent uniformly distributed random variables, too. He showed that almost every scenery can be almost surely reconstructed. In [12], Kesten noticed that this proof in [18] heavily relies on the skip-free property of the random walk. He asked whether the result might still hold in the case of a random walk with jumps. This article gives a positive answer to Kesten's question: If the random walk can reach every integer with positive probability and is recurrent with bounded jumps, and if there are strictly more colors than possible single steps for the random walk, then one can almost surely reconstruct almost every scenery up to equivalence.

More formally: Let $\mathcal{C}=\left\{1, \ldots, C_{0}\right\}$ denote the set of colors. Let $\mu$ be a probability measure over $\mathbb{Z}$ supported over a finite set $\mathcal{M}:=\operatorname{supp} \mu \subseteq \mathbb{Z}$. With respect to a probability measure $P$, let $S=(S(k))_{k \in \mathbb{N}}$ be a random walk starting in the origin and with independent increments having the distribution $\mu$. We assume that $E[S(1)]=0$; thus $S$ is recurrent. Furthermore we assume that $\operatorname{supp} \mu$ has the greatest common divisor 1 , thus $S$ can reach every $z \in \mathbb{Z}$ with positive probability. Let $\xi=(\xi(j))_{j \in \mathbb{Z}}$ be a family of i.i.d. random variables, independent of $S$, uniformly distributed over $\mathcal{C}$. We prove:

Theorem 1.1 If $|\mathcal{C}|>|\mathcal{M}|$, then there exists a measurable map $\mathcal{A}: \mathcal{C}^{\mathbb{N}} \rightarrow \mathcal{C}^{\mathbb{Z}}$ such that

$$
P[\mathcal{A}(\chi) \approx \xi]=1 .
$$

Research on random sceneries started by work by Keane and den Hollander [10], [4]. They thoroughly investigated ergodic properties of a color record seen along a random walk. These questions were motivated among others by the work of Kalikow [9] and den Hollander, Steif [3], in ergodic theory.

As was shown in [19] the two color scenery reconstruction problem for a scenery which is i.i.d. is equivalent to the following problem: let $(R(k))_{k \in \mathbb{Z}}$ and $(S(k))_{k \geq 0}$ be two independent simple random walks on $\mathbb{Z}$ both starting at the origin and living on the same probability space. Does one path realization of the iterated random walk $(R(S(k)))_{k \geq 0}$ uniquely determine the path of $(R(k))_{k \in \mathbb{Z}}$ a.s. up to shift and reflection around the origin? This is a discrete time analogue to a problem solved by Burdzy [2] concerning the path of iterated Brownian motion.

A preform of the scenery reconstruction problem is the problem of distinguishing two given sceneries. It has been investigated by Benjamini and Kesten in [1] and [11]. Howard in a series of articles [8], [7], [6] also contributed to this area; see below. The scenery distinguishing problem 
is the following: Given two different sceneries $\xi, \xi^{\prime}$ and observations $(\tilde{\xi}(S(j)))_{j \geq 0}$, where $\tilde{\xi}$ equals either $\xi$ or $\xi^{\prime}$, the question is: Can we distinguish whether $\tilde{\xi}=\xi$ or $\tilde{\xi}=\xi^{\prime}$ ? Benjamini and Kesten [1] showed that one can almost surely distinguish almost all pairs of sceneries $\xi$, $\xi^{\prime}$, if they are drawn independently with i.i.d. entries. Their result even holds in the two dimensional case. This result is not beaten by a reconstruction result: the reconstruction method in two dimensions by Löwe and Matzinger [15] holds only when we have many colors. When $\xi$ and $\xi^{\prime}$ differ in precisely one point, the distinguishing problem was examined by Kesten [11] and Howard [6]. Kesten proved that almost all pairs of those sceneries $\left(\xi, \xi^{\prime}\right)$ can be distinguished in the 5 -color case. He assumes the sceneries to be i.i.d. Howard proved that all periodic sceneries can be distinguished.

As mentioned above, it is in general not possible to reconstruct $\xi$; one can at most expect a reconstruction up to equivalence. As a matter of fact, even this is impossible: By a theorem of Lindenstrauss [14], there exist non-equivalent sceneries that cannot be distinguished. Of course, they also cannot get reconstructed.

For sceneries that can be reconstructed Benjamini asked whether the reconstruction works also in polynomial time. This question was positively answered by Matzinger and Rolles [21] and [23] (see also [20]) in the case of a two color scenery and a simple random walk with holding. Löwe and Matzinger [16] proved that reconstruction works in many cases even if the scenery is not i.i.d., but has some correlations. For the setting of our article den Hollander asked if the finite bound on the length of the jumps is necessary for scenery reconstruction.

In a way a result by Lenstra and Matzinger complements the present paper. If the random walk might jump more than distance 1 only with very small probability and if the tail of the distribution of the jumps decays sufficiently fast, Lenstra and Matzinger [13] proved that scenery reconstruction is still possible.

Based on the results of the present paper, Matzinger and Rolles [22] showed that the scenery can be still reconstructed if there are some random errors in the observations.

Let us explain how this article is organized. In order to avoid getting lost among the many details of the rather complex proof, this article is ordered in a "top-down" approach: In order to show the global structure of the reconstruction procedure in a compact but formal way, we start with a section called "Skeleton". This section collects the main theorems and main definitions of the reconstruction method, using "lower level" procedures as black boxes. In the "Skeleton" section, we only show how these theorems fit together to yield a proof of the reconstruction theorem 1.1; all proofs of the "ingredient" theorems are postponed to later sections. Although this approach is more abstract than a "bottom-up" structure would be, we hope that it allows the reader to more quickly see the global structure.

Overview on some steps for the reconstruction procedure The reconstruction starts with an ergodicity argument: It suffices to consider only sceneries which produce a very untypical initial piece of observations; in particular we may condition on a large but finite initial piece of the observations to be constant. We apply a reconstruction procedure, which works only in this untypical situation, again and again to the observations with larger and larger initial pieces 
dropped, disregarding all instances that do not produce the prescribed "untypical" outcome. Finally we will see even the prescribed "untypical situation" sufficiently frequently to successfully reconstruct the scenery. The "untypical initial piece" serves to identify locations close to the origin at later times again, at least up to a certain time horizon.

The reconstruction procedure consists of a hierarchy of partial reconstruction procedures; these try to reconstruct larger and larger pieces of the scenery around the origin. The hierarchy of partial reconstruction procedures is defined recursively.

To reconstruct a large piece in the $(m+1)$ st hierarchical level, we need some information where the random walker is located while producing its color records. This information is encoded in stopping times, which stop the random walk with high probability sufficiently close to the origin, at least up to a certain time horizon.

The stopping times for the $(m+1)$ st hierarchical level are built using the $m$ th level partial reconstruction procedure: Given a reconstructed piece around the origin from the $m$ th level, one starts the whole $m$ th level partial reconstruction procedure again at a later "candidate time". Whenever the piece of scenery obtained in this way has a sufficiently high overlap with the reconstructed piece around the origin, then one has a high chance that the random walk is close to the origin at the "candidate time".

The global structure of this recursive construction is formally described in the "Skeleton" Section 3. Most theorems in this section are proven in Section 5, and we prove in Sections 7 and 8 that the stopping times fulfill their specification. Some related lemmas claimed in Section 5 are also proved in Section 8.

The heart of the reconstruction procedure, i.e. the construction of the partial reconstruction algorithm given the stopping times, is described in Section 4 and proven to be correct in Section 6. Roughly speaking, to reconstruct a piece of scenery of size $2^{n}$, we collect a "puzzle" of words of size proportional to $n$, i.e. logarithmically in the size of the piece to be reconstructed. The puzzle contains (with high probability) all correct subwords of the given size in the "true" piece of scenery to be reconstructed, but also some "garbage" words. We play a kind of puzzle game with these pieces: starting with seed words, we reconstruct larger and larger pieces by adjoining more and more pieces of the puzzle that fit to the growing piece.

Although the actual construction is much more complicated than the idea described now, let us describe an (over)simplified version of how to collect pieces in the puzzle: Suppose we have two "characteristic signals" $A$ and $B$ in the scenery, which occur only once in the scenery. Suppose that the distance between $A$ and $B$ is a multiple of the maximal step size $l_{\rightarrow}$ of the random walk to the right. Then we can almost surely identify the whole "ladder" word read while stepping from $A$ to $B$ with step size $l_{\rightarrow}$ as follows: Look at all occurrences of $A$ and $B$ in the color record with minimal distance. The words occurring in the color record between those $A$ and $B$ should (a.s.) be always the same in the whole record, and it is the "ladder" word we are looking for. Of course, by ergodicity there are almost surely no (bounded) signals $A$ and $B$ in the scenery that occur only once; this is why the simple idea described here cannot be applied without considerable refinement.

The "pieces of puzzle" obtained are $l_{\rightarrow}$-spaced pieces; not pieces with spacing 1 . This is why our puzzle game leads to reconstructions of modulo classes of the scenery modulo $l_{\rightarrow}$ only. In order to successfully reconstruct the whole scenery, we need to arrange these modulo classes correctly, using some "neighborship" relation between pieces of the puzzle. Unfortunately, the correct arrangement of modulo classes is a technically intricate step in the reconstruction procedure. 


\section{Some notation}

We collect some globally used nonstandard notations and conventions in this section.

Sets, functions, and integers: For functions $f$ and sets $D$ the notation $f\lceil D$ means the restriction of $f$ to the set $D$. $D$ need not be contained in the domain of $f$; thus $f\lceil D$ is defined on $D \cap$ domain $(f)$. If $f$ and $g$ are functions, the notation $f \subseteq g$ means that $f$ is a restriction of $g$; this notation is consistent with the set theoretic definition of functions. By convention, $0 \in \mathbb{N}$. The integer part of a real number $r$ is denoted by $\lfloor r\rfloor:=\max \{z \in \mathbb{Z} \mid z \leq r\}$; similarly $\lceil r\rceil:=\min \{z \in \mathbb{Z} \mid z \geq r\}$.

Integer intervals: Unless explicitly stated otherwise, intervals are taken over the integers, e.g. $[a, b]=\{n \in \mathbb{Z}: a \leq n \leq b\},] a, b\left[=\{n \in \mathbb{Z}: a<n<b\}\right.$. Given a fixed number $C_{0}$, we define the set of colors $\mathcal{C}:=\left[1, C_{0}\right]=\left\{1, \ldots, C_{0}\right\},|\mathcal{C}|=C_{0}$.

In the rest of this section $I$ will denote an arbitrary subset of $\mathbb{Z}$ unless otherwise specified.

Sceneries and equivalence: By definition, a scenery is an element of $\mathcal{C}^{\mathbb{Z}}$. If $I \subseteq \mathbb{Z}$, then the elements of $\mathcal{C}^{I}$ are called pieces of scenery. The length $|\zeta|$ of a piece of scenery $\zeta \in \mathcal{C}^{I}$ is the cardinality $|I|$ of its index set. $\zeta^{\leftrightarrow}:=\left(\zeta_{-i}\right)_{i \in-I}$ denotes the reflection of a piece of scenery $\zeta \in \mathcal{C}^{I}$ at the origin. Two pieces of scenery $\zeta \in \mathbb{Z}^{I}$ and $\zeta^{\prime} \in \mathbb{Z}^{I^{\prime}}$ are called strongly equivalent, $\zeta \equiv \zeta^{\prime}$, if $\zeta$ is obtained by some translation of $\zeta^{\prime}$, i.e. $I^{\prime}=I+b$ for some $b \in \mathbb{Z}$, and $\zeta=\left(\zeta_{i+b}^{\prime}\right)_{i \in I}$. $\zeta$ and $\zeta^{\prime}$ are called equivalent, $\zeta \approx \zeta^{\prime}$, if $\zeta$ is obtained by some translation or reflection of $\zeta^{\prime}$, i.e. $I^{\prime}=a I+b$ for some $a \in\{ \pm 1\}, b \in \mathbb{Z}$, and $\zeta=\left(\zeta_{a i+b}^{\prime}\right)_{i \in I}$. If $T: \mathbb{Z} \rightarrow \mathbb{Z}, T(z)=a z+b$, denotes this translation or reflection, then $T[\zeta]:=\zeta^{\prime}$ denotes the transport of $\zeta^{\prime}$ by $T$; the same notation is used for the domains: $T[I]=I^{\prime}$. By definition, $\zeta \preccurlyeq \zeta^{\prime}$ means that $\zeta \approx \zeta^{\prime}\left\lceil J\right.$ for some $J \subseteq I^{\prime}$. If additionally such a subset $J \subseteq I^{\prime}$ and its reading direction (i.e. either $\zeta \equiv \zeta^{\prime}\left\lceil J\right.$ or $\zeta \equiv\left(\zeta^{\prime}\lceil J) \leftrightarrow\right.$ ) is unique, we write $\zeta \preccurlyeq 1 \zeta^{\prime}$. Similarly $\zeta \sqsubseteq \zeta^{\prime}$ (in words: " $\zeta$ occurs in $\zeta^{\prime \prime}$ ") means that $\zeta \equiv \zeta^{\prime}\lceil J$ for some $J \subseteq I^{\prime}$.

Words: The elements of $\mathcal{C}^{*}:=\bigcup_{n \in \mathbb{N}} \mathcal{C}^{n}=\bigcup_{n \in \mathbb{N}} \mathcal{C}^{\{0, \ldots, n-1\}}$ are called words (over $\mathcal{C}$ ). We identify $\mathcal{C}$ with $\mathcal{C}^{1}$. The concatenation of two words $w_{1} \in \mathcal{C}^{n}$ and $w_{2} \in \mathcal{C}^{m}$ is denoted by $w_{1} w_{2} \in \mathcal{C}^{n+m}$.

Probability distributions: The law of a random variable $X$ with respect to a probability measure $P$ is denoted by $\mathcal{L}_{P}(X)$. The $n$-fold convolution of a probability distribution $\mu$ over $\mathbb{R}$ is denoted by $\mu^{* n}$.

Random sceneries and random walks: As mentioned before, let $\mu$ be a probability measure over $\mathbb{Z}$ supported over a finite set $\mathcal{M}=\operatorname{supp} \mu \subseteq \mathbb{Z}$. Let $\Omega_{2} \subseteq \mathbb{Z}^{\mathbb{N}}$ denote the set of all paths with starting point $S(0)=0$ and jump sizes $S(t+1)-S(t) \in \mathcal{M}, t \in \mathbb{N}$. Let $Q_{0}$ denote the law of a random walk $S=(S(k))_{k \in \mathbb{N}}$ with start in $0 \in \mathbb{Z}$ and with independent increments having the distribution $\mu$. Furthermore, let $\xi=\left(\xi_{j}\right)_{j \in \mathbb{Z}}$ be a family of i.i.d. random variables, independent of $S$, with uniform distribution $\mathcal{L}\left(\xi_{j}\right)=\nu$ over $\mathcal{C}$. We realize $(\xi, S)$ as canonical projections of $\Omega=\mathcal{C}^{\mathbb{Z}} \times \Omega_{2}$ endowed with its canonical product $\sigma$-algebra and the probability measure $P:=\nu^{\mathbb{Z}} \otimes Q_{0}$. (The restriction of the random walk paths not to have forbidden jumps even on null sets is technically convenient.) We assume that $E[S(1)-S(0)]=0(k \in \mathbb{N})$; 
thus $S$ is recurrent. Furthermore we assume that $\operatorname{supp} \mu$ has the greatest common divisor 1, thus $S$ eventually reaches every $z \in \mathbb{Z}$ with probability one. For fixed sceneries $\xi \in \mathcal{C}^{\mathbb{Z}}$, we set $P_{\xi}:=\delta_{\xi} \otimes Q_{0}$, where $\delta_{\xi}$ denotes the Dirac measure at $\xi$. Thus $P_{\xi}$ is the "canonical" version of the conditional probability $P[\cdot \mid \xi]$. We use the notations $P_{\xi}$ and $P[\cdot \mid \xi]$ as synonyms; i.e. we will never work with a different version of the conditional measure $P[\cdot \mid \xi]$ than $P_{\xi}$.

Filtrations: We define the filtration $\mathcal{F}:=\left(\mathcal{F}_{n}\right)_{n \in \mathbb{N}}, \mathcal{F}_{n}:=\sigma\left(\xi,(S(k))_{k=0, \ldots, n}\right)$ over $\Omega$. We further introduce the filtration $\mathcal{G}:=\left(\mathcal{G}_{n}\right)_{n \in \mathbb{N}}$ over $\mathcal{C}^{\mathbb{N}}$, where $\mathcal{G}_{n}$ is the $\sigma$-algebra generated by the projection map $\mathcal{C}^{\mathbb{N}} \rightarrow \mathcal{C}^{[0, n]}, \chi \mapsto \chi\lceil[0, n]$.

Observations of the scenery along the random walk and shifts: Let $\chi=\left(\chi_{n}\right)_{n \in \mathbb{N}}:=$ $\left(\xi_{S(n)}\right)_{n \in \mathbb{N}}$. We sometimes write simply $\chi=\xi \circ S$; this is to be understood in the sense $\chi(\omega)=$ $\xi(\omega) \circ S(\omega)$ for all $\omega \in \Omega$. Let $\mathcal{H}=\left(\mathcal{H}_{n}\right)_{n \in \mathbb{N}}, \mathcal{H}_{n}:=\sigma\left(\chi_{k}, 0 \leq k \leq n\right)$ denote the filtration obtained by observing the scenery along initial pieces of the random walk. We define the shift operations $\theta: \mathcal{C}^{\mathbb{N}} \rightarrow \mathcal{C}^{\mathbb{N}},\left(\chi_{n}\right)_{n \in \mathbb{N}} \mapsto\left(\chi_{n+1}\right)_{n \in \mathbb{N}}$, and $\Theta: \Omega \rightarrow \Omega,(\xi, S) \mapsto\left(\left(\xi_{n+S(1)}\right)_{n \in \mathbb{Z}},(S(k+\right.$ 1) $-S(1))_{k \in \mathbb{N}}$; thus $\chi \circ \Theta=\theta \circ \chi$. Intuitively, $\Theta$ spatially shifts both the scenery and the random walk by the location $S(1)$ of the random walk after one step, and it drops the first time step. One observes $\xi \approx \xi \circ \Theta$.

Admissible paths: A piece of path $\pi=\left(\pi_{i}\right)_{i \in I} \in \mathbb{Z}^{I}$ over an integer interval $I$ is called admissible if $\pi_{i+1}-\pi_{i} \in \mathcal{M}$ for all $\{i, i+1\} \subseteq I$. For finite $I \neq \emptyset, \pi_{\min I}$ and $\pi_{\max I}$ are called starting point and end point of $\pi$, respectively. We set $\operatorname{TimeShift}(\pi):=\left(\pi_{i-1}\right)_{i \in I+1}$. By definition, the length $|\pi|$ of the path $\pi$ is the cardinality $|I|$. For $x, t>0$ let $\operatorname{AdPaths}(x, t)$ denote the set of all admissible pieces of path $\pi \in[-x, x]^{[0, t[}$.

Ladder intervals and ladder paths: $\quad$ Let $l_{\rightarrow}:=\max \mathcal{M}, l_{\leftarrow}:=|\min \mathcal{M}|$; thus $l_{\rightarrow}$ and $l_{\leftarrow}$ are the maximal possible jump sizes of $S$ to the right and to the left, respectively. We abbreviate $l:=\max \left\{l_{\rightarrow}, l_{\leftarrow}\right\}$ and $h:=l|\mathcal{M}|$. By definition, $d$-spaced intervals $(d \in \mathbb{N})$ are sets of the form $I \cap(a+d \mathbb{Z})$ with a bounded interval $I$ and a modulo class $a+d \mathbb{Z} \in \mathbb{Z} / d \mathbb{Z}$. $l_{\rightarrow}$-spaced intervals are

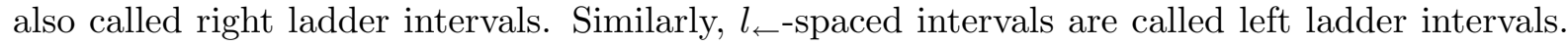
By definition, a right ladder path is a piece of path that steps through the points of some right ladder interval in increasing order. Similarly, a left ladder path is a piece of path that steps through the points of some left ladder interval in decreasing order.

Reading words from pieces of sceneries: For $I=\left\{i_{0}, \ldots, i_{n-1}\right\} \subset \mathbb{Z}$ with $i_{0}<\ldots<i_{n-1}$ and a piece of scenery $\zeta \in \mathcal{C}^{I}$, we define $\zeta_{\rightarrow}:=\left(\zeta_{i_{k}}\right)_{k=0, \ldots, n-1} \in \mathcal{C}^{n}$ and $\zeta_{\leftarrow}:=\left(\zeta_{i_{n-1-k}}\right)_{k=0, \ldots, n-1} \in$ $\mathcal{C}^{n}$; thus $\zeta_{\rightarrow}$ and $\zeta_{\leftarrow}$ are the words obtained by reading $\zeta$ from the left to the right and from the right to the left, respectively. The right ladder word of a scenery $\xi$ over a right ladder interval $I$ is defined to be $\left(\xi\lceil I)_{\rightarrow}\right.$; similarly one defines left ladder words $\left(\xi\lceil J)_{\leftarrow}\right.$ over left ladder intervals $J$.

\subsection{Conventions concerning constants}

Four fixed "sufficiently large" positive integer parameters $c_{2}, c_{1}, \alpha$, and $n_{0}$ globally play a role. The meaning of these parameters is explained below at the location of their occurrence; at this point we only describe their mutual dependence: 
- $c_{2} \in \mathbb{N}$ is chosen first sufficiently large; say $c_{2} \geq c_{2}^{\min }(|\mathcal{C}|, \mu)$.

- Then $c_{1} \in 2 \mathbb{N}$ is chosen to be even and sufficiently large; say $c_{1} \geq c_{1}^{\min }\left(c_{2},|\mathcal{C}|, \mu\right)$.

- Then $\alpha \in \mathbb{N}$ is chosen to be sufficiently large; say $\alpha \geq \alpha^{\min }\left(c_{1},|\mathcal{C}|, \mu\right)$.

- Finally $n_{0} \in 2 \mathbb{N}$ is chosen to be even and sufficiently large; say $n_{0} \geq n_{0}^{\min }\left(c_{1}, \alpha,|\mathcal{C}|, \mu\right)$.

We do not specify explicitly here how large the allowed lower bounds $c_{2}^{\min }, c_{1}^{\min }, \alpha^{\mathrm{min}}$ and $n_{0}^{\min }$ actually need to be; but we emphasize that the constructions below will work if they are sufficiently large.

All other positive constants are denoted by " $c_{i}$ " with a counting index $i>2$; they keep their meaning globally during the whole article. Unless explicitly stated otherwise, these constants may depend only on the number of colors $|\mathcal{C}|$ and on the jump distribution $\mu$ of the random walk; in particular they may depend on the upper bound $l$ of the jump size, but not on $n_{0}$.

\section{Skeleton of the Reconstruction Procedure}

Our first "ingredient" theorem reduces the problem of almost surely reconstructing sceneries to the following simpler one: We only need to find an auxiliary reconstruction procedure $\mathcal{A}_{\mathrm{B}}$ which may fail to give an answer, and it may sometimes even give the wrong answer, if only giving the correct answer is more probable than giving a wrong one. Roughly speaking, we apply the auxiliary reconstruction procedure $\mathcal{A}_{\mathrm{B}}$ repeatedly to the observations with initial pieces dropped, taking the answer of the majority as our result; here ergodicity of the observations plays a key role.

Theorem 3.1 If there exists a measurable map $\mathcal{A}_{\mathrm{B}}: \mathcal{C}^{\mathbb{N}} \rightarrow \mathcal{C}^{\mathbb{Z}} \cup\{$ fail $\}$ with

$$
P\left[\mathcal{A}_{\mathrm{B}}(\chi) \neq \text { fail, } \mathcal{A}_{\mathrm{B}}(\chi) \approx \xi\right]>P\left[\mathcal{A}_{\mathrm{B}}(\chi) \neq \text { fail, } \mathcal{A}_{\mathrm{B}}(\chi) \not \approx \xi\right],
$$

then there exists a measurable map $\mathcal{A}: \mathcal{C}^{\mathbb{N}} \rightarrow \mathcal{C}^{\mathbb{Z}}$ such that

$$
P[\mathcal{A}(\chi) \approx \xi]=1
$$

The auxiliary reconstruction procedure $\mathcal{A}_{\mathrm{B}}$ gives the output "fail" if one does not see a long block of 1 's in the initial piece of the observations. Thus failure of $\mathcal{A}_{\mathrm{B}}$ is a very frequent event; however, non-failure still occurs with a positive but small probability, and conditioned on this event the most probable answer will be the correct one. Roughly speaking, when we apply $\mathcal{A}_{\mathrm{B}}$ again and again to the observations with initial pieces dropped, we will finally see sufficiently many long blocks of 1's to make the whole procedure work correctly.

The required long block of 1 's in the initial piece should have length $n_{0}^{20}$ for some sufficiently large but fixed even number $n_{0} \in 2 \mathbb{N}$. The parameter $n_{0}$, which parametrizes the size of this required block, is chosen fixed but large enough (see Subsection 2.1).

Definition 3.2 With the abbreviation $J_{1}=\left[-2 \ln _{0}^{20}, 2 \ln n_{0}^{20}\right]$, we define the following events:

$$
\begin{aligned}
E_{\mathrm{B}}(k) & :=\left\{\chi_{n}=1 \text { for all } n \leq k\right\} \text { for } k \in \mathbb{N}, \\
\text { BigBlock } & :=\left\{\begin{array}{l}
\text { There is an integer interval } J_{0} \subseteq J_{1} \text { with }\left|J_{0}\right| \geq n_{0}^{4} \text { such that } \\
\xi\left\lceil J_{0}=(1)_{j \in J_{0}} \text { is a constant piece of scenery with value } 1 .\right.
\end{array}\right\} .
\end{aligned}
$$


Let $P_{\mathrm{B}}$ denote the image of the conditional law $P\left[\cdot \mid E_{\mathrm{B}}\left(n_{0}^{20}\right)\right]$ with respect to the shift $\Theta^{n_{0}^{20}}$. Furthermore, we define the conditional law

$$
\tilde{P}:=P_{\mathrm{B}}[\cdot \mid \text { BigBlock }] \text {. }
$$

The event $E_{\mathrm{B}}\left(n_{0}^{20}\right)$ occurs when we see a large block of 1's in an initial piece of the observations, while BigBlock occurs when there is a large block of 1's close to the origin in the (unobservable) real scenery $\xi$.

We describe the intuitive meaning of $\tilde{P}$ : After having seen a large initial block of 1's in the observations, we drop this initial piece and take the present point as our new starting point. Since then a large block of 1's close to the origin in the unobservable real scenery $\xi$ is typical, it does not change much when we even condition on this (unobservable) event.

The next theorem shows that whenever we have a reconstruction procedure $\mathcal{A}^{\prime}$ that works sufficiently probably with respect to the modified measure $\tilde{P}$, then there exists the auxiliary reconstruction procedure $\mathcal{A}_{\mathrm{B}}$ that we needed above:

Theorem 3.3 Assume that there exists a measurable map $\mathcal{A}^{\prime}: \mathcal{C}^{\mathbb{N}} \rightarrow \mathcal{C}^{\mathbb{Z}}$ with

$$
\tilde{P}\left[\mathcal{A}^{\prime}(\chi) \approx \xi\right] \geq \frac{2}{3}
$$

Then there exists a measurable map $\mathcal{A}_{\mathrm{B}}: \mathcal{C}^{\mathbb{N}} \rightarrow \mathcal{C}^{\mathbb{Z}} \cup\{$ fail $\}$ such that

$$
P\left[\mathcal{A}_{\mathrm{B}}(\chi) \neq \text { fail, } \mathcal{A}_{\mathrm{B}}(\chi) \approx \xi\right]>P\left[\mathcal{A}_{\mathrm{B}}(\chi) \neq \text { fail, } \mathcal{A}_{\mathrm{B}}(\chi) \not \approx \xi\right] .
$$

The reconstruction function $\mathcal{A}^{\prime}$ required by the last theorem is built by putting together a hierarchy of partial reconstruction algorithms $\mathcal{A}^{m}, m \geq 1$. The partial reconstruction algorithms $\mathcal{A}^{m}$ try to reconstruct longer and longer pieces around the origin; the relevant length scale in the $m$-th hierarchy is given by $2^{n_{m}}$, where $n_{m}$ is defined as follows:

Definition 3.4 We define recursively a sequence $\left(n_{m}\right)_{m \in \mathbb{N}}: n_{0}$ was already chosen above; we set

$$
n_{m+1}:=2^{\left\lfloor\sqrt{n_{m}}\right\rfloor} .
$$

The partial reconstruction algorithms may sometimes, but not too frequently, give the wrong answer:

Theorem 3.5 Assume that there exists a sequence $\left(\mathcal{A}^{m}\right)_{m \geq 1}$ of measurable maps $\mathcal{A}^{m}: \mathcal{C}^{\mathbb{N}} \rightarrow$ $\mathcal{C}^{\left[-5 \cdot 2^{n_{m}}, 5 \cdot 2^{n_{m}}\right]}$ such that

$$
\tilde{P}\left[\bigcup_{m=1}^{\infty}\left(E^{m}\right)^{\mathrm{c}}\right] \leq \frac{1}{3},
$$

where

$$
E^{m}:=\left\{\xi \left\lceil\left[-2^{n_{m}}, 2^{n_{m}}\right] \preccurlyeq \mathcal{A}^{m}(\chi) \preccurlyeq \xi\left\lceil\left[-9 \cdot 2^{n_{m}}, 9 \cdot 2^{n_{m}}\right]\right\} .\right.\right.
$$

Then there exists a measurable map $\mathcal{A}^{\prime}: \mathcal{C}^{\mathbb{N}} \rightarrow \mathcal{C}^{\mathbb{Z}}$ such that the following holds :

$$
\tilde{P}\left[\mathcal{A}^{\prime}(\chi) \approx \xi\right] \geq \frac{2}{3}
$$


Before describing it formally, let us intuitively explain how the hierarchy of partial reconstruction algorithms $\mathcal{A}^{m}$ is constructed: The $\mathcal{A}^{m}$ are built recursively in a "zig-zag" way simultaneously with a hierarchy of stopping times:

These stopping times have the task to estimate times when the random walk $S$ is sufficiently close back to the origin, at least up to a certain time horizon. For this estimation, one may use only an initial piece of the color record $\chi$. To find "higher level" stopping times, we try to reconstruct a piece of scenery both at the present candidate location and at the starting point, using a "lower level" partial reconstruction algorithm. If the two obtained pieces of scenery have a high overlap with each other, then there is a good chance that the candidate location and the starting point are close to each other. This is the "zig" part of the "zig-zag" recursion.

The "zag" part of the recursion uses the stopping times as follows to construct a "higher level" partial reconstruction algorithm $\mathcal{A}^{m}$ : Whenever the stopping times indicate that one might be sufficiently close to the origin, one collects "typical signals" which one expects to be characteristic of the local environment in the scenery. The data obtained in this way are then matched together similarly to playing a puzzle game. This procedure is the heart of the whole reconstruction method.

To get the whole construction started, one needs some initial stopping times which indicate that one might be sufficiently close to the origin. A simple way to get such times is the following: Whenever one observes a sufficiently long block of 1's in the color record, then one has a high chance to be close to the origin. (Remember: We conditioned on seeing a long block of 1's at an initial piece of the color record.) This is the reason why we introduce the modified measure $\tilde{P}$, since with respect to $\tilde{P}$ one can be (almost) sure to have a big block of 1 's in the scenery close to the origin. However, the such constructed stopping times are not reliable enough to base the first partial reconstruction algorithm on them. Instead, these stopping times are used as ingredients to construct more reliable stopping times.

We treat the "zig" part and the "zag" part of the recursion separately, starting with the formal specification of the "zig" part: Given an abstract partial reconstruction algorithm $f$, we build stopping times out of it:

The specification of the stopping times depends on a fixed, sufficiently large parameter $\alpha \in \mathbb{N}$. Informally speaking, $\alpha$ influences how many stopping times in each step should be valuable, and what the time horizon for the $m$-th partial reconstruction algorithm in the hierarchy should be. The parameter $\alpha$ is chosen fixed but large enough; recall Subsection 2.1.

Definition 3.6 Let $m \geq 1$. Let a function $f: \mathcal{C}^{\mathbb{N}} \rightarrow \mathcal{C}^{\left[-5 \cdot 2^{n_{m}}, 5 \cdot 2^{n_{m}}\right]}$ be given. Assume that $f(\chi)$ depends only on $\chi\left\lceil\left[0,2 \cdot 2^{12 \alpha n_{m}}[\right.\right.$. We define the random set

$$
\mathbb{T}_{f}(\chi):=\left\{t \in \left[0,2^{12 \alpha n_{m+1}}-2 \cdot 2^{12 \alpha n_{m}}\left[\mid \exists w \in \mathcal{C}^{2 \cdot 2^{n_{m}}}: w \preccurlyeq f(\chi) \text { and } w \preccurlyeq f\left(\theta^{t}(\chi)\right)\right\} .\right.\right.
$$

We define a sequence $T_{f}=\left(T_{f, k}\right)_{k>0}$ of $\mathcal{G}$-adapted stopping times with values in $\left[0,2^{12 \alpha n_{m+1}}\right]$ : Let $t(0)<\ldots<t\left(\left|\mathbb{T}_{f}(\chi)\right|-1\right)$ be the elements of $\mathbb{T}_{f}(\chi)$ arranged in increasing order. For $k \in \mathbb{N}$, we set

$$
T_{f, k}(\chi):= \begin{cases}t\left(2 \cdot 2^{2 n_{m+1}} k\right)+2 \cdot 2^{12 \alpha n_{m}} & \text { if } 2 \cdot 2^{2 n_{m+1}} k<\left|\mathbb{T}_{f}(\chi)\right| \\ 2^{12 \alpha n_{m+1}} & \text { otherwise }\end{cases}
$$

Observe that the stopping times $T_{f}(\chi)$ depend only on $\chi\left\lceil\left[0,2^{12 \alpha n_{m+1}}[\right.\right.$.

In the next definition, we introduce events $E_{\mathrm{stop}, \tau}^{m}$; they specify what the stopping times should fulfill: There should be sufficiently many of them, they should be separated by at least 
$2 \cdot 2^{2 n_{m}}$, and they should stop the random walk sufficiently close to the origin. Furthermore, given any abstract partial reconstruction algorithm $f$, we define an event $E_{\text {reconst, } f}^{m}$; it measures whether $f$ correctly reconstructs a piece of the scenery around the origin.

Definition 3.7 Let $m \in \mathbb{N}$.

1. Given a sequence $\tau=\left(\tau_{k}\right)_{k \in \mathbb{N}}$ of $\mathcal{G}$-adapted stopping times, we define

$$
E_{\text {stop }, \tau}^{m}:=\bigcap_{k=0}^{2^{\alpha n_{m}}}\left\{\tau_{k}(\chi)<2^{12 \alpha n_{m}},\left|S\left(\tau_{k}(\chi)\right)\right| \leq 2^{n_{m}}, \tau_{j}(\chi)+2 \cdot 2^{2 n_{m}} \leq \tau_{k}(\chi) \text { for } j<k\right\} .
$$

2. We set for $f: \mathcal{C}^{\mathbb{N}} \rightarrow \mathcal{C}^{\left[-5 \cdot 2^{n_{m}}, 5 \cdot 2^{n_{m}}\right]}$ :

$$
E_{\text {reconst }, f}^{m}:=\left\{\xi \left\lceil\left[-2^{n_{m}}, 2^{n_{m}}\right] \preccurlyeq f(\chi) \preccurlyeq \xi\left[\left[-9 \cdot 2^{n_{m}}, 9 \cdot 2^{n_{m}}\right]\right\} .\right.\right.
$$

Roughly speaking, the following theorem states: there are stopping times "to get started" which solve their task with high probability:

Theorem 3.8 There exists a sequence of $\mathcal{G}$-adapted stopping times $T^{1}=\left(T_{k}^{1}\right)_{k \in \mathbb{N}}$ with values in $\left[0,2^{12 \alpha n_{1}}\right]$ and a constant $c_{4}>0$, such that

$$
\tilde{P}\left[\left(E_{\text {stop }, T^{1}}^{1}\right)^{\mathrm{c}}\right] \leq e^{-c_{4} n_{0}} .
$$

The next theorem states that the "zig"-part of the construction works correctly with high probability. As a premise, the "zig"-part needs the underlying "lower level" partial reconstruction algorithm $f$ to work correctly when $f$ is applied at the beginning. Furthermore, the "zig"-part needs $f$ to have a sufficiently high probability to work correctly on the given scenery $\xi$ whenever it is applied again. Informally speaking, the reason is: In the "zig"-part we can only reconstruct, if we know where we are. The idea is to start the whole lower-level reconstruction procedure again whenever we want to find out whether we are close to the origin. As mentioned before, if the result has a large overlap with the piece we have already reconstructed, we can be rather sure that we are close to the origin.

Theorem 3.9 Under the assumptions of Definition 3.6, we have that

$$
P\left[\left(E_{\text {stop }, T_{f}}^{m+1}\right)^{\mathrm{c}} \cap E_{\text {reconst }, f}^{m} \cap\left\{P\left[E_{\text {reconst }, f}^{m} \mid \xi\right] \geq \frac{1}{2}\right\}\right] \leq e^{-n_{m+1}} .
$$

We remark: in the "zig part" (Theorem 3.9) we work with the event $E_{\text {stop }, T_{f}}^{m+1}$, while in the "zag part" (Theorem 3.10 below) we work with $E_{\text {stop, } T_{f}}^{m}$.

Intuitively, in order to successfully recognize locations close to the origin, we need not only the "lower level" reconstruction to work correctly the first time (i.e. $E_{\text {reconst, } f}^{m}$ needs to hold), but also the scenery must be such that whenever one starts the "lower level" reconstruction again, one has a sufficiently high chance to reconstruct again a correct piece; this is why we need the event " $P\left[E_{\text {reconst }, f}^{m} \mid \xi\right] \geq 1 / 2$ ".

Finally the heart of the reconstruction algorithm consists of the "zag"-part: there are partial reconstruction algorithms $\mathrm{Alg}^{n_{m}}$ which take an initial piece of the color record as input data, and abstract "lower level" stopping times $\tau$ as "argument procedures". Intuitively, the following theorem states that the algorithms $\operatorname{Alg}^{n_{m}}$ reconstruct correctly with high probability, provided the "argument procedures" $\tau$ fulfill their specification $E_{\mathrm{stop}, \tau}^{m}$. 
Theorem 3.10 For every $m \in \mathbb{N}$, there is a map

$$
\operatorname{Alg}^{n_{m}}:\left[0,2^{12 \alpha n_{m}}\right]^{\mathbb{N}} \times \mathcal{C}^{2 \cdot 2^{12 \alpha n_{m}}} \rightarrow \mathcal{C}^{\left[-5 \cdot 2^{n_{m}}, 5 \cdot 2^{n_{m}}\right]}
$$

such that for every vector $\tau=\left(\tau_{k}\right)_{k \in \mathbb{N}}$ of $\mathcal{G}$-adapted stopping times with values in $\left[0,2^{12 \alpha n_{m}}\right]$ one has

$$
P\left[\left(E_{\text {reconst }, \operatorname{Alg}^{n_{m}}(\tau, \cdot)}^{m}\right)^{\mathrm{c}} \cap E_{\text {stop }, \tau}^{m}\right] \leq c_{5} e^{-c_{6} n_{m}}
$$

for some positive constants $c_{6}$ and $c_{5}$, where $\operatorname{Alg}^{n_{m}}(\tau, \cdot): \chi \mapsto \operatorname{Alg}^{n_{m}}\left(\tau(\chi), \chi\left\lceil\left[0,2 \cdot 2^{12 \alpha n_{m}}[)\right.\right.\right.$.

To motivate the allowed range for the abstract arguments $\tau$ in this theorem, recall that $T_{f, k}(\chi)$ in (3.13) take their values in $\left[0,2^{12 \alpha n_{m+1}}\right]$.

Note that Theorems 3.9 and 3.10 use the original probability measure $P$, while Theorem 3.8 uses the modified probability measure $\tilde{P}$.

An algorithm $\operatorname{Alg}^{n}$ is defined in the next Section 4, but its correctness, i.e. Theorem 3.10, is proven in Section 6, below. Theorems 3.9 and 3.8 are proven below in separate Sections 7 and 8, respectively. Right now we show how to use these three theorems: Provided these three theorems are true, the hypothesis of Theorem 3.5 holds, i.e. there exists a sequence of measurable maps $\mathcal{A}^{m}: \mathcal{C}^{\mathbb{N}} \rightarrow \mathcal{C}^{\left[-5 \cdot 2^{n_{m}}, 5 \cdot 2^{n_{m}}\right]}$ such that (3.9) is valid. We take the maps $\operatorname{Alg}^{n_{m}}$ and the sequences of stopping times $T^{1}, T_{f}$ from Theorems $3.8,3.9$, and 3.10 to define recursively maps $\mathcal{A}^{m}$. Then we prove: the properties guaranteed by Theorems 3.8, 3.9, and 3.10 imply that the sequence of maps $\left(\mathcal{A}^{m}\right)_{m \geq 1}$ satisfies (3.9). We are ready to describe the "zig-zag"-recursion formally:

Definition 3.11 We define $\mathcal{A}^{m}: \mathcal{C}^{\mathbb{N}} \rightarrow \mathcal{C}^{\left[-5 \cdot 2^{n m}, 5 \cdot 2^{n m}\right]}$ and sequences $T^{m}=\left(T_{k}^{m}\right)_{k \in \mathbb{N}}$ of $\mathcal{G}$ adapted stopping times by simultaneous recursion over $m \geq 1$ :

- $T^{1}$ is chosen using Theorem 3.8 .

- $\mathcal{A}^{m}(\chi):=\operatorname{Alg}^{n_{m}}\left(T^{m}(\chi), \chi\left\lceil\left[0,2 \cdot 2^{12 \alpha n_{m}}[)\right.\right.\right.$, with $\operatorname{Alg}^{n_{m}}$ taken from Theorem 3.10.

- $T^{m+1}:=T_{\mathcal{A}^{m}}$, with the notation of Definition 3.6.

Recall Definition (3.10) of the events $E^{m}$. From now on, we use our specific choice for $\mathcal{A}^{m}$ from Definition 3.11. Using (3.15), we rewrite (3.10) in the form

$$
E^{m}=E_{\text {reconst }, \mathcal{A}^{m}}^{m}
$$

Theorem 3.12 For the sequence $\left(\mathcal{A}^{m}\right)_{m \geq 1}$ as defined in Definition 3.11 and $\left(E^{m}\right)_{m \in \mathbb{N}}$ as in (3.20), the bound (3.9) is valid.

All theorems of this section together yield the proof of our main theorem:

Proof of Theorem 1.1. By Theorem 3.12, (3.9) holds; then (3.11) holds by Theorem 3.5; moreover (3.7) holds by Theorem 3.3; finally Theorem 3.1 implies the claim (1.1) of Theorem 1.1. 


\section{Heart of the Reconstruction Procedure: Definition of the Algorithm $\mathrm{Alg}^{n}$}

This section contains the heart of the reconstruction procedure: for every $n \in \mathbb{N}$, we define an algorithm $\mathrm{Alg}^{n}$; it is designed to reconstruct long pieces of scenery with high probability. In Section 6 below we show that it fulfills the formal specification given in Theorem 3.10.

Informally speaking, the observation $\chi$ allows us to collect many pieces of "puzzle words". These puzzle words are chosen to have size $c_{1} n$ with a fixed parameter $c_{1}$; recall Subsection 2.1. To obtain them, we collect triples of words $\left(w_{1}, w_{2}, w_{3}\right)$ which occur in sequence in the observations $\chi$ soon after a stopping time $\tau(k)$; an initial piece of $\chi$ is represented below by a formal argument $\eta$. We put those words $w_{2}$ into our puzzle which are already uniquely determined by $w_{1}$ and $w_{3}$. This means that $w_{1}$ and $w_{3}$ should be be very "characteristic signals"; if $w_{1}$ and $w_{3}$ could be read at very different locations in the scenery close to a stopping time, then it is improbable that they will enclose always the same word $w_{2}$. Frequently, $w_{2}$ turns out to be a ladder word: Whenever one reads a $w_{2}$ in the context $w_{1} w_{2} w_{3}$ along a non-ladder path sufficiently close to the origin, one reads with high probability a different word $w_{2}^{\prime}$ in the context $w_{1} w_{2}^{\prime} w_{3}$, too, along a different path with the same starting point and the same end point; but then $w_{2}$ is not collected as a puzzle word.

Here is the formal construction: We take input data $\tau \in\left[0,2^{12 \alpha n}\right]^{\mathbb{N}}$ and $\eta \in \mathcal{C}^{2 \cdot 2^{12 \alpha n}}$. A side remark: although for formal reasons there are infinitely many $\tau(k)$ given in the input data, the construction below actually uses only the first $2^{\alpha n}$ of them.

Definition 4.1 We define for $m \geq 0$ the random sets:

$$
\begin{aligned}
& \operatorname{PrePuzzle}^{n}(\tau, \eta):= \\
& \left\{\left(w_{1}, w_{2}, w_{3}\right) \in\left(\mathcal{C}^{c_{1} n}\right)^{3} \mid \exists k \in\left[0,2^{\alpha n}\left[: w_{1} w_{2} w_{3} \sqsubseteq \eta\left\lceil\left[\tau(k), \tau(k)+2^{2 n}\right]\right\},\right.\right.\right. \\
& \operatorname{Puzzle}_{\mathrm{I}}^{n}(\tau, \eta):= \\
& \left\{\left(w_{1}, w_{2}, w_{3}\right) \in \operatorname{PrePuzzle}^{n}(\tau, \eta) \mid \forall\left(w_{1}, w_{2}^{\prime}, w_{3}\right) \in \operatorname{PrePuzzle}^{n}(\tau, \eta): w_{2}^{\prime}=w_{2}\right\}, \\
& \operatorname{Puzzle}_{\mathrm{II}}^{n}(\tau, \eta):= \\
& \left\{w_{2} \in \mathcal{C}^{c_{1} n} \mid \exists w_{1}, w_{3} \in \mathcal{C}^{c_{1} n}:\left(w_{1}, w_{2}, w_{3}\right) \in \operatorname{Puzzle}_{\mathrm{I}}^{n}(\tau, \eta)\right\} .
\end{aligned}
$$

Let us informally explain why for the reconstruction to work we need more colors then possible steps of the random walk: A ladder word should be very characteristic for the location where it occurs, at least within a large neighborhood of the origin. To illustrate this, take a "typical" finite sequence $c_{1}, \ldots, c_{l}$ of colors in $\mathcal{C}$ in the observation $\chi$. Look at all locations $x$ in the scenery $\xi$ where one can observe this sequence $c_{1}, \ldots, c_{l}$ of colors by following some admissible path starting at $x$. As $l \rightarrow \infty$, we want that these locations $x$ to get very rare. However, this will not be the case if there are too few possible colors, compared to the number $|\mathcal{M}|$ of possible steps of the random walk. More formally, the condition $|\mathcal{C}|>|\mathcal{M}|$ on the number of possible colors is used in Lemma 6.38, below.

Let us explain the idea behind the following constructions: Although many of the words $w_{2}$ in "Puzzle II" turn out to be ladder words of a central piece in the true scenery $\xi$, some of them are not: There are "garbage words" in the puzzle. We play a "puzzle-game" with the words in "Puzzle II": We try to fit larger and larger pieces together. In order to distinguish "real" pieces from "garbage" pieces, we need some "seed words" which are guaranteed (with high probability) 
not to be garbage words; every piece that fits to a piece containing a seed word has a high chance not to be garbage, too. This is what the set Seed II defined below is good for. We identify "seed" words as "puzzle" words that occur in the observations almost immediately after a stopping time $\tau(k)$, when we expect the random walk to be close to the origin.

Recall the abbreviation $h=l|\mathcal{M}|$. Formally, we proceed as follows:

\section{Definition 4.2}

$$
\begin{aligned}
& \operatorname{Seed}_{\mathrm{I}}^{n}(\tau, \eta):= \\
& \left\{\left(w_{1}, w_{2}, w_{3}\right) \in \operatorname{Puzzle}_{\mathrm{I}}^{n}(\tau, \eta) \mid \begin{array}{c}
\exists k \in\left[0,2^{\alpha n}\left[\exists j \in\left[0,7 c_{1} n l\right]:\right.\right. \\
w_{1} w_{2} w_{3} \equiv \eta\left\lceil\left(\tau(k)+j+\left[0,3 c_{1} n[)\right.\right.\right.
\end{array}\right\}, \\
& \operatorname{Seed}_{\mathrm{II}}^{n}(\tau, \eta):=\left\{w_{2} \in \mathcal{C}^{c_{1} n} \mid\left(w_{1}, w_{2}, w_{3}\right) \in \operatorname{Seed}_{\mathrm{I}}^{n}(\tau, \eta)\right\}, \\
& \operatorname{Seed}_{\mathrm{III}}^{n}(\tau, \eta):= \\
& \left\{u \in \operatorname{Seed}_{\mathrm{II}}^{n}(\tau, \eta) \mid \begin{array}{l}
\exists v \in \operatorname{Seed}_{\mathrm{II}}^{n}(\tau, \eta): \\
\left(u\left\lceil\left(\left[0, c_{2} n l_{\leftarrow}\right] \cap l_{\leftarrow} \mathbb{Z}\right)\right)_{\rightarrow}=\left(v\left\lceil\left(\left[0, c_{2} n l_{\rightarrow}\right] \cap l_{\rightarrow} \mathbb{Z}\right)\right)_{\leftarrow}\right.\right.
\end{array}\right\}, \\
& \operatorname{Neighbors}^{n}(\tau, \eta):= \\
& \left\{\left(w_{1}, w_{2}\right) \in\left(\mathcal{C}^{c_{1} n}\right)^{2} \mid \exists k \in\left[0,2^{\alpha n}\left[, w \in \mathcal{C}^{h-1}: w_{1} w w_{2} \sqsubseteq \eta\left\lceil\left[\tau(k), \tau(k)+2^{2 n}\right]\right\} .\right.\right.\right.
\end{aligned}
$$

Let us explain what "Seed III" is intended for: We need to identify the orientation of the pieces (whether they are to be read "forward" or "backward"). This task consists of two problems: The identification of the relative orientation of two pieces with respect to each other, and the identification of the absolute orientation with respect to the "true" scenery $\xi$. Of course, we have no chance to identify the absolute orientation if the random walk is symmetric; we even bother about identifying the absolute orientation only in the very unsymmetric case $l_{\rightarrow} \neq l_{\leftarrow}$. The set Seed III helps us to identify the absolute orientation in this case: Suppose we read every $l_{\rightarrow}$-th letter in a word from the left to the right, and every $l_{\leftarrow}$-th letter in the same word from the right to the left; then every $l_{\rightarrow} l_{\leftarrow}$-th letter appears in both words, when at least one letter is read both times. This turns out to be characteristic enough to identify the reading directions "left" and "right" in the case $l_{\rightarrow} \neq l_{\leftarrow}$. The fixed parameter $c_{2}$ influences the length of the sample pieces in this procedure.

The relation "Neighbors" serves as an estimation for the geometric neighborship relation between ladder words: ladder words that occur closely together in the observation $\chi$ are expected to occur on geometrically neighboring intervals in the "true" scenery $\xi$. The next definition defines a "true" geometric neighborship relation $\triangleright_{n}$. We try to reconstruct the corresponding "true" neighborship relation for ladder words in a piece of $\xi$ using only the "estimated" neighborship relation "Neighbors".

Recall that $\mu^{* k}$ denotes the $k$-fold convolution of $\mu$; in particular

$$
\operatorname{supp} \mu^{* k}:=\left\{\sum_{i=1}^{k} s_{i} \mid \forall i: s_{i} \in \operatorname{supp} \mu\right\} .
$$

Definition 4.3 Let $I, J$ be right ladder intervals. By definition, $I \triangleright_{n} J$ means $|I|=|J|=c_{1} n$ and $\min J-\max I \in \operatorname{supp} \mu^{* h}$. Similarly for $I^{\prime}, J^{\prime}$ being left ladder intervals, $I^{\prime} \triangleleft_{n} J^{\prime}$ means $\left|I^{\prime}\right|=\left|J^{\prime}\right|=c_{1} n$ and $\max J^{\prime}-\min I^{\prime} \in \operatorname{supp} \mu^{* h}$. 
The next definition is the heart of our method: We describe how to obtain reconstructed pieces of sceneries. All pieces of scenery $w \in \mathcal{C}^{\left[-5 \cdot 2^{n}, 5 \cdot 2^{n}\right]}$ are tested as candidates in a sequence of "Filters": Reconstructed ladder words should be in "Puzzle II", the "estimated" and the "reconstructed" neighborship relation should be consistent with each other, the reconstructed pieces should contain "Seed III" words, and no piece of the puzzle should be used twice.

Only candidate pieces that pass all Filters are considered as a solution of the partial reconstruction problem.

Definition 4.4 Let $\operatorname{Filter}_{i}^{n}(\tau, \eta), i=1, \ldots, 5$, denote the set of all $w \in \mathcal{C}^{\left[-5 \cdot 2^{n}, 5 \cdot 2^{n}\right]}$ which fulfill the following condition $1 ., \ldots, 5 .$, respectively:

1. For every right ladder interval $I \subseteq\left[-5 \cdot 2^{n}, 5 \cdot 2^{n}\right],|I|=c_{1} n$, one has $\left(w\lceil I) \rightarrow \in \operatorname{Puzzle}_{\mathrm{II}}^{n}(\tau)\right.$.

2. For all right ladder intervals $I, J \subseteq\left[-5 \cdot 2^{n}, 5 \cdot 2^{n}\right]$ :

if $I \triangleright_{n} J$, then $\left(\left(w\lceil I)_{\rightarrow},\left(w\lceil J)_{\rightarrow}\right) \in\right.\right.$ Neighbors $^{n}(\tau, \eta)$.

3. For all right ladder intervals $I, J \subseteq\left[-5 \cdot 2^{n}, 5 \cdot 2^{n}\right],|I|=|J|=c_{1} n$ : if $\left(\left(w\lceil I)_{\rightarrow},\left(w\lceil J)_{\rightarrow}\right) \in\right.\right.$ Neighbors $^{n}(\tau, \eta)$, then there is $q \in \mathbb{N}$ such that $I \triangleright_{n} J+q l_{\rightarrow}$.

4. For every right modulo class $Z \in \mathbb{Z} / l_{\rightarrow} \mathbb{Z}$ there exists a right ladder interval $I \subseteq Z \cap[-2$. $\left.2^{n}, 2 \cdot 2^{n}\right]$ such that $\left(w\lceil I)_{\rightarrow} \in \operatorname{Seed}_{\mathrm{III}}^{n}(\tau, \eta)\right.$.

5. For all right ladder intervals $I, J \subseteq\left[-5 \cdot 2^{n}, 5 \cdot 2^{n}\right],|I|=|J|=c_{1} n$ : if $\left(w\lceil I)_{\rightarrow}=\left(w\lceil J)_{\rightarrow}\right.\right.$, then $I=J$.

We set

$$
\text { SolutionPieces }^{n}(\tau, \eta):=\bigcap_{i=1}^{5} \operatorname{Filter}_{i}^{n}(\tau, \eta) .
$$

The output of the algorithm $\operatorname{Alg}^{n}$ could be any of these pieces $w \in$ SolutionPieces $^{n}(\tau, \eta)$; we choose one of them, if it exists.

Definition 4.5 We define $\operatorname{Alg}^{n}(\tau, \eta)$ as follows:

- If SolutionPieces ${ }^{n}(\tau, \eta)$ is nonempty, then we define $\operatorname{Alg}^{n}(\tau, \eta)$ to be its lexicographically smallest element.

- Otherwise $\operatorname{Alg}^{n}(\tau, \eta)$ is defined to be the constant scenery $(1)_{j \in\left[-5 \cdot 2^{n}, 5 \cdot 2^{n}\right]}$.

We could have equally well taken any element of SolutionPieces ${ }^{n}(\tau, \eta)$ in Definition 4.5 ; we choose the lexicographically smallest one just for definiteness.

\section{$5 \quad$ Proofs concerning the Skeleton Structure}

In this section, we prove most of the theorems of Section 3, which deals with the skeleton structure.

We start with a quite standard lemma:

Lemma 5.1 The shift $\Theta: \Omega \rightarrow \Omega,(\xi, S) \mapsto(\xi(\cdot+S(1)), S(\cdot+1)-S(1))$ is measure-preserving and ergodic with respect to $P$. 
On page 397 in [9], Kalikov remarks (without proof) that the shift operator in a more general situation is K, not only ergodic. However, for completeness, we describe a proof of the lemma. We are grateful to an anonymous referee for a simplification in the subsequent proof of ergodicity.

Proof. The shift $\Theta$ is measure-preserving: Since the distribution of $\xi$ is invariant under (deterministic) translations, and since $S(1)$ is independent of $\xi$, we get: $\xi(\cdot+S(1))$ has the same distribution as $\xi$. Furthermore, $(S(t+1)-S(1))_{t \in \mathbb{N}}$ has the same distribution as $S$. Since $\xi, S(1)$ and $(S(t+1)-S(1))_{t \in \mathbb{N}}$ are independent, $\xi(\cdot+S(1))$ and $(S(t+1)-S(1))_{t \in \mathbb{N}}$ are independent, too. Consequently $\Theta(\xi, S)$ has the same distribution as $(\xi, S)$.

To prove that $\Theta$ is ergodic, let $A$ be a $\Theta$-invariant event. Given $\epsilon>0$, there is $N \in \mathbb{N}$ and an event $B_{\epsilon}$ with $P\left[A \triangle B_{\epsilon}\right]<\epsilon$, such that $B_{\epsilon}$ depends only on the first $N$ steps $S(1), \ldots, S(N)$ of the random walk and the piece of the scenery $(\xi(i))_{-N \leq i \leq N}$ close to the origin. Here $A \triangle B_{\epsilon}$ denotes the symmetric difference of sets. Using $A \triangle\left(B_{\epsilon} \cap \Theta^{-n} B_{\epsilon}\right) \subseteq\left(A \triangle B_{\epsilon}\right) \cup \Theta^{-n}\left(A \triangle B_{\epsilon}\right)$, we get $\left|P\left[B_{\epsilon} \cap \Theta^{-n} B_{\epsilon}\right]-P[A]\right|<2 \epsilon$ (uniformly) for all $n \in \mathbb{N}$. On the other hand, $\lim _{n \rightarrow \infty} P\left[B_{\epsilon} \cap\right.$ $\left.\Theta^{-n} B_{\epsilon}\right]=P\left[B_{\epsilon}\right]^{2}$, since after many time steps with high probability, the random walk is very far from the origin: $\lim _{n \rightarrow \infty} P[|S(n)|>C]=1$ for all $C>0$, and conditioned on $|S(n)|>C$ for sufficiently large $C=C(N)$, the events $B_{\epsilon}$ and $\Theta^{-n} B_{\epsilon}$ are independent. Thus

$$
P[A]^{2}=\lim _{\epsilon \rightarrow 0} P\left[B_{\epsilon}\right]^{2}=\lim _{\epsilon \rightarrow 0} \lim _{n \rightarrow \infty} P\left[B_{\epsilon} \cap \Theta^{-n} B_{\epsilon}\right]=P[A],
$$

i.e. $P[A] \in\{0,1\}$.

Proof of Theorem 3.1. The idea of this proof is to apply the reconstruction function $\mathcal{A}_{B}$ to all the shifted observations $\theta^{k}(\chi)$ for each $k \in \mathbb{N}$. Every time one does this, one gets either a scenery or the state fail as result.

Given $\mathcal{A}_{B}: \mathcal{C}^{\mathbb{N}} \rightarrow \mathcal{C}^{\mathbb{Z}} \cup\{$ fail $\}$ as in the hypothesis of the theorem, we define measurable functions $\mathcal{A}_{B}^{k}: \mathcal{C}^{\mathbb{N}} \rightarrow \mathcal{C}^{\mathbb{Z}}, k \in \mathbb{N}$, as follows:

- If there exists $j \in\left[0, k\left[\right.\right.$ such that $\mathcal{A}_{B}\left(\theta^{j}(\chi)\right) \neq$ fail and

$$
\begin{aligned}
& \mid\left\{j ^ { \prime } \in \left[0, k\left[\mid \mathcal{A}_{B}\left(\theta^{j^{\prime}}(\chi)\right) \neq \text { fail, } \mathcal{A}_{B}\left(\theta^{j^{\prime}}(\chi)\right) \approx \mathcal{A}_{B}\left(\theta^{j}(\chi)\right)\right\} \mid\right.\right. \\
> & \mid\left\{j ^ { \prime } \in \left[0, k\left[\mid \mathcal{A}_{B}\left(\theta^{j^{\prime}}(\chi)\right) \neq \text { fail, } \mathcal{A}_{B}\left(\theta^{j^{\prime}}(\chi)\right) \not \approx \mathcal{A}_{B}\left(\theta^{j}(\chi)\right)\right\} \mid,\right.\right.
\end{aligned}
$$

then let $j_{0}$ be the smallest $j$ with this property, and define $\mathcal{A}_{B}^{k}(\chi):=\mathcal{A}_{B}\left(\theta^{j_{0}}(\chi)\right)$.

- Else define $\mathcal{A}_{B}^{k}(\chi)$ to be the constant scenery $(1)_{j \in \mathbb{Z}}$.

Finally define the measurable function $\mathcal{A}: \mathcal{C}^{\mathbb{N}} \rightarrow \mathcal{C}^{\mathbb{Z}}$ by

$$
\mathcal{A}(\chi):= \begin{cases}\lim _{k \rightarrow \infty} \mathcal{A}_{B}^{k}(\chi) & \text { if this limit exists pointwise } \\ (1)_{j \in \mathbb{Z}} & \text { otherwise. }\end{cases}
$$

We check that the such defined function $\mathcal{A}$ fulfills the claim (3.1) of Theorem 3.1:

Let us give the general idea: by hypothesis (3.1) and an ergodicity argument, "on the long run" the proportion of sceneries $\mathcal{A}_{B}\left(\theta^{k}(\chi)\right.$ ) (for $k \in \mathbb{N}$ ) which are equivalent to $\xi$ is strictly bigger than the proportion of sceneries which are not equivalent to $\xi$. More formally, define for $k \in \mathbb{Z}$ the Bernoulli variables $X_{\text {sce }}^{k}$ and $X_{\text {wrong sce }}^{k}$ we set $X_{\text {sce }}^{k}$ equal to 1 iff $\mathcal{A}_{B}\left(\theta^{k}(\chi)\right) \neq$ fail and $\mathcal{A}_{B}\left(\theta^{k}(\chi)\right) \approx \xi$. Similarly, $X_{\text {wrong sce }}^{k}$ is equal to 1 iff $\mathcal{A}_{B}\left(\theta^{k}(\chi)\right) \neq$ fail and $\mathcal{A}_{B}\left(\theta^{k}(\chi)\right) \not \approx \xi$. Define

$$
Y_{\mathrm{sce}}^{k}:=\frac{1}{k} \sum_{i=0}^{k-1} X_{\mathrm{sce}}^{i} \quad \text { and } \quad Y_{\mathrm{wrong} \text { sce }}^{k}:=\frac{1}{k} \sum_{i=0}^{k-1} X_{\mathrm{wrong} \text { sce }}^{i}
$$


Observe that if $Y_{\text {sce }}^{k}>Y_{\text {wrong sce }}^{k}$ holds, then $\mathcal{A}_{B}^{k}(\chi) \approx \xi$. As a consequence of Lemma 5.1, the sequences $\left(X_{\text {sce }}^{k}\right)_{k \geq 0}$ and $\left(X_{\text {wrong sce }}^{k}\right)_{k \geq 0}$ are stationary and ergodic, since they can be viewed as a measurable function of the sequence $k \mapsto \Theta^{k}(\xi, S)$. Note that $\xi \approx \xi(\cdot+S(k))$. By the ergodic theorem, we have almost surely:

$$
\begin{aligned}
Y_{\mathrm{sce}}^{k} & \stackrel{k \rightarrow \infty}{\longrightarrow} P\left[\mathcal{A}_{B}\left(\theta^{k}(\chi)\right) \neq \text { fail, } \mathcal{A}_{B}(\chi) \approx \xi\right], \\
Y_{\text {wrong sce }}^{k} & \stackrel{k \rightarrow \infty}{\longrightarrow} P\left[\mathcal{A}_{B}\left(\theta^{k}(\chi)\right) \neq \text { fail, } \mathcal{A}_{B}(\chi) \not \xi\right] .
\end{aligned}
$$

Thus by the assumption (3.1) there exists a.s. a (random) $k_{0}$ such that for all $k \geq k_{0}$ we have $Y_{\text {sce }}^{k}>Y_{\text {wrong sce }}^{k}$ and hence $\mathcal{A}_{B}^{k}(\chi)=\mathcal{A}_{B}^{k_{0}}(\chi) \approx \xi$; recall that we chose the smallest possible $j_{0}$ in the definition of $\mathcal{A}_{B}^{k}$. Thus a.s. $\mathcal{A}(\chi) \approx \xi$.

The following lemma tells us that a large block of 1's in the real scenery is very probable whenever we see a large initial block of 1's in the observations. It is proven in Section 8.1, below.

Lemma 5.2 There exists $c_{3}>0$ such that $P_{\mathrm{B}}[$ BigBlock $] \geq 1-e^{-c_{3} n_{0}^{12}}$.

Proof of Theorem 3.3. Assume $\mathcal{A}^{\prime}: \mathcal{C}^{\mathbb{N}} \rightarrow \mathcal{C}^{\mathbb{Z}}$ is a measurable map satisfying (3.6):

$$
P_{\mathrm{B}}\left[\mathcal{A}^{\prime}(\chi) \approx \xi \mid \text { BigBlock }\right] \geq \frac{2}{3} .
$$

So,

$$
P_{\mathrm{B}}\left[\left\{\mathcal{A}^{\prime}(\chi) \approx \xi\right\} \cap \text { BigBlock }\right] \geq \frac{2}{3} P_{\mathrm{B}}[\text { BigBlock }] .
$$

By Lemma 5.2 it follows, since $n_{0}$ is large enough (see Subsection 2.1):

$$
P_{\mathrm{B}}\left[\mathcal{A}^{\prime}(\chi) \approx \xi\right] \geq \frac{2}{3}\left(1-e^{-c_{3} n_{0}^{12}}\right)>\frac{1}{2} .
$$

Now, by definition of $P_{\mathrm{B}}$,

$$
P_{\mathrm{B}}\left[\mathcal{A}^{\prime}(\chi) \approx \xi\right]=P\left[\mathcal{A}^{\prime}\left(\chi \circ \Theta^{n_{0}^{20}}\right) \approx \xi \circ \Theta^{n_{0}^{20}} \mid E_{\mathrm{B}}\left(n_{0}^{20}\right)\right] .
$$

Obviously $\xi \circ \Theta^{n_{0}^{20}} \approx \xi$. Thus

$$
P\left[\mathcal{A}^{\prime}\left(\chi \circ \Theta^{n_{0}^{20}}\right) \approx \xi \mid E_{\mathrm{B}}\left(n_{0}^{20}\right)\right]>\frac{1}{2} .
$$

We define $\mathcal{A}_{\mathrm{B}}: \mathcal{C}^{\mathbb{N}} \rightarrow \mathcal{C}^{\mathbb{Z}} \cup\{$ fail $\}:$

$$
\mathcal{A}_{\mathrm{B}}(\chi):= \begin{cases}\mathcal{A}^{\prime}\left(\chi \circ \Theta^{n_{0}^{20}}\right) & \text { if } E_{\mathrm{B}}\left(n_{0}^{20}\right) \text { holds, } \\ \text { fail } & \text { otherwise }\end{cases}
$$

this is well defined since $E_{\mathrm{B}}\left(n_{0}^{20}\right) \in \sigma(\chi)$. By (5.11), the such defined $\mathcal{A}_{\mathrm{B}}$ satisfies (3.7).

The following lemma claims that $\tilde{P}$ is absolutely continuous with respect to $P$, and it provides an upper bound for the Radon-Nikodym derivative $d \tilde{P} / d P$. This lemma is also proven in Section 8.1, below.

Lemma 5.3 For all events $E \subseteq \Omega$ we have

$$
\tilde{P}(E) \leq|\mathcal{C}|^{4 \ln _{0}^{20}+1} P(E) .
$$


Proof of Theorem 3.5. For pieces of scenery $\psi, \phi$, we define the piece of scenery $\Phi(\psi, \phi)$ as follows: If $\psi \preccurlyeq 1 \phi$, then $\Phi(\psi, \phi)$ denotes the unique piece of scenery with $\Phi(\psi, \phi) \approx \phi$ such that $\psi \subseteq \Phi(\psi, \phi)$; otherwise we set $\Phi(\psi, \phi):=\phi$. We take $\mathcal{A}^{m}$ as in the hypothesis of the theorem and $\chi \in \mathcal{C}^{\mathbb{N}}$. With the abbreviation $\xi^{m}:=\mathcal{A}^{m}(\chi)$, we define recursively

$$
\begin{aligned}
\zeta^{1} & :=\xi^{1} \\
\zeta^{m+1} & :=\Phi\left(\zeta^{m}, \xi^{m+1}\right), \\
\mathcal{A}^{\prime}(\chi) & := \begin{cases}\lim _{m \rightarrow \infty} \zeta^{m} & \text { if this limit exists pointwise on } \mathbb{Z}, \\
(1)_{j \in \mathbb{Z}} & \text { else. }\end{cases}
\end{aligned}
$$

(By convention, a sequence $\left(\zeta^{m}\right)_{m \in \mathbb{N}}$ of pieces of sceneries converges pointwise to a scenery $\zeta$ if the following holds: $\liminf \operatorname{in}_{m \rightarrow \infty} \operatorname{domain}\left(\zeta^{m}\right)=\mathbb{Z}$, and for every $z \in \mathbb{Z}$ there is $m_{z}>0$ such that for all $m \geq m_{z}$ one has $\zeta^{m}(z)=\zeta(z)$.) Being a pointwise limit of measurable maps, the map $\mathcal{A}^{\prime}: \mathcal{C}^{\mathbb{N}} \rightarrow \mathcal{C}^{\mathbb{Z}}$ is measurable. For the purpose of the proof, we abbreviate $\underline{\xi}^{m}:=\xi\left\lceil\left[-2^{n_{m}}, 2^{n_{m}}\right]\right.$ and $\bar{\xi}^{m}:=\xi\left\lceil\left[-9 \cdot 2^{n_{m}}, 9 \cdot 2^{n_{m}}\right]\right.$ and we define the events

$$
E_{1 \mathrm{fit}}^{m}:=\left\{\underline{\xi}^{m} \preccurlyeq{ }_{1} \bar{\xi}^{m+1}\right\}
$$

We claim:

1. $\liminf \operatorname{in}_{m \rightarrow \infty} E_{1 \text { fit }}^{m}$ holds $\tilde{P}$-a.s.,

2. If the event $\liminf _{m \rightarrow \infty} E_{1 \text { fit }}^{m} \cap \bigcap_{m=1}^{\infty} E^{m}$ occurs, then $\mathcal{A}^{\prime}(\chi) \approx \xi$.

These two statements together with the hypothesis (3.9) imply the claim (3.11) of the theorem.

Proof of claim 1.: By Lemma 5.3 we may replace " $\tilde{P}$-a.s." in the claim by " $P$-a.s.". If $I_{1} \neq I_{2}$ are fixed integer intervals with $\left|I_{1}\right|=\left|I_{2}\right|$, then $P\left[\xi\left\lceil I_{1} \approx \xi\left\lceil I_{2}\right] \leq 2 c_{12} e^{-c_{13}|I|}\right.\right.$ holds for some constants $c_{12}, c_{13}>0$, even if $I_{1}$ and $I_{2}$ are not disjoint. (See also the similar Lemma 6.33, in particular estimate (6.66), below. The factor 2 makes the notation consistent with this lemma; recall the binary choice: $\xi\left\lceil I_{1} \approx \xi\left\lceil I_{2}\right.\right.$ means $\xi\left\lceil I_{1} \equiv \xi\left\lceil I_{2}\right.\right.$ or $\xi\left\lceil I_{1} \equiv\left(\xi\left\lceil I_{2}\right) \leftrightarrow\right.\right.$.) We apply this for $I_{1}=\left[-2^{n_{m}}, 2^{n_{m}}\right]$ and all integer intervals $I_{2} \subseteq\left[-9 \cdot 2^{n_{m+1}}, 9 \cdot 2^{n_{m+1}}\right]$ with $\left|I_{1}\right|=\left|I_{2}\right|=2 \cdot 2^{n_{m}}+1$, $I_{1} \neq I_{2}$; there are at most $18 \cdot 2^{n_{m+1}}$ choices of $I_{2}$. We obtain $P\left[\left(E_{1 \mathrm{fit}}^{m}\right)^{\mathrm{c}}\right] \leq 18 \cdot 2^{n_{m+1}} \cdot 2 c_{12} e^{-2 c_{13} 2^{n_{m}}}$, which is summable over $m$; recall $n_{m+1}=o\left(2^{n_{m}}\right)$ as $m \rightarrow \infty$. Hence $\left(E_{1 \text { fit }}^{m}\right)^{\mathrm{c}}$ occurs $P$-a.s. only finitely many times by the Borel-Cantelli lemma; this proves claim 1.

Next we prove the second claim: By the assumption made there, there is a (random) $M$ such that the events $E_{1 \text { fit }}^{m}$ and $E^{m}$ hold for all $m \geq M$. Let $m \geq M$. In the considerations below, we use several times the following rule: For pieces of sceneries $\alpha, \beta, \gamma, \delta$ :

$$
\text { If } \alpha \preccurlyeq \beta \preccurlyeq \gamma \preccurlyeq \delta \text { and } \alpha \preccurlyeq 1 \delta \text {, then } \beta \preccurlyeq 1 \gamma \text {. }
$$

In particular, this applies to

$$
\underline{\xi}^{m} \preccurlyeq 1 \bar{\xi}^{m+1} \text { and } \quad \underline{\xi}^{m} \preccurlyeq \xi^{m} \preccurlyeq \bar{\xi}^{m} \preccurlyeq \underline{\xi}^{m+1} \preccurlyeq \xi^{m+1} \preccurlyeq \bar{\xi}^{m+1} ;
$$

we obtain $\xi^{m} \preccurlyeq 1 \xi^{m+1}$. By the definition of $\zeta^{m}$ and $\Phi$, we know $\zeta^{m} \approx \xi^{m}$; hence we obtain $\zeta^{m} \preccurlyeq 1 \xi^{m+1}$. Using the definition of $\Phi$ again, we see $\zeta^{m} \subseteq \Phi\left(\zeta^{m}, \xi^{m+1}\right)=\zeta^{m+1}$. Using (5.18), (5.19), $\zeta^{m} \approx \xi^{m}, \zeta^{m+1} \approx \xi^{m+1}$ again, we get

$$
\zeta^{m} \preccurlyeq 1 \bar{\xi}^{m} \preccurlyeq 1 \zeta^{m+1} \preccurlyeq 1 \bar{\xi}^{m+1} \text { and } \zeta^{m} \preccurlyeq 1 \bar{\xi}^{m+1} \text {. }
$$


Let $h^{m}: \mathbb{Z} \rightarrow \mathbb{Z}, m \geq M$, denote the unique translation or reflection that maps $\zeta^{m}$ onto a subpiece of $\bar{\xi}^{m}$. As a consequence of $\zeta^{m} \subseteq \zeta^{m+1}, \bar{\xi}^{m} \subseteq \bar{\xi}^{m+1}$, and (5.20) we see that $h^{m}$ does not depend on $m$ for $m \geq M$. Hence $h^{m}$ maps $\zeta:=\bigcup_{m \geq M} \zeta^{m}$ to a subpiece of $\xi=\bigcup_{m \geq M} \bar{\xi}^{m}$; thus $\zeta \preccurlyeq \xi$. In fact the domain of $\zeta$ is $\mathbb{Z}$; to see this we observe that domain $(\zeta)$ contains all $\left(h^{m}\right)^{-1}\left[\operatorname{domain}\left(\bar{\xi}^{m}\right)\right]=\left(h^{m}\right)^{-1}\left[-9 \cdot 2^{n_{m}}, 9 \cdot 2^{n_{m}}\right]$, which cover all of $\mathbb{Z}$. To summarize, we have shown that $\left(\zeta^{m}\right)_{m \geq M}$ converges pointwise to a scenery $\zeta \approx \xi$; thus $\mathcal{A}^{\prime}(\chi)=\zeta \approx \xi$ by the definition of $\mathcal{A}^{\prime}(\chi)$. This finishes the proof of the second claim and also the proof of Theorem 3.5 .

Definition 5.4 We define events of sceneries

$$
\begin{aligned}
\Xi_{\mathrm{I}} & :=\left\{\xi \in \mathcal{C}^{\mathbb{Z}} \mid P\left[\left(E_{\mathrm{stop}, T^{1}}^{1}\right)^{\mathrm{c}} \mid \xi\right] \leq e^{-c_{4} n_{0} / 2}\right\} \\
\Xi_{\mathrm{II}} & :=\bigcap_{m=1}^{\infty}\left\{\xi \in \mathcal{C}^{\mathbb{Z}} \mid \text { If } P\left[E^{m} \mid \xi\right] \geq \frac{1}{2}, \text { then } P\left[\left(E_{\mathrm{stop}, T^{m+1}}^{m+1} \cap E^{\mathrm{c}} \mid \xi\right] \leq e^{-n_{m+1} / 2}\right\}\right. \\
& =\bigcap_{m=1}^{\infty}\left\{\xi \in \mathcal{C}^{\mathbb{Z}} \mid P\left[\left(E_{\mathrm{stop}, T^{m+1}}^{m+1}\right)^{\mathrm{c}} \cap E^{m} \cap\left\{P\left[E^{m} \mid \xi\right] \geq \frac{1}{2}\right\} \mid \xi\right] \leq e^{-n_{m+1} / 2}\right\},(5.22) \\
\Xi_{\mathrm{III}} & :=\bigcap_{m=1}^{\infty}\left\{\xi \in \mathcal{C}^{\mathbb{Z}} \mid P\left[\left(E^{m}\right)^{\mathrm{c}} \cap E_{\mathrm{stop}, T^{m}}^{m} \mid \xi\right] \leq c_{5}^{1 / 2} e^{-c_{6} n_{m} / 2}\right\} \\
\Xi & :=\Xi_{\mathrm{I}} \cap \Xi_{\mathrm{II}} \cap \Xi_{\mathrm{III}},
\end{aligned}
$$

where $c_{5}$ and $c_{6}$ are taken from Theorem 3.10 and $c_{4}$ is taken from Theorem 3.8.

Note the similarity between these events and the bounds in (3.16), (3.17) and (3.19). The following lemma provides a link between bounds with and without conditioning on the scenery $\xi$ :

Lemma 5.5 Let $A$ be an event, $r \geq 0$, and $Q$ be a probability measure on $\Omega$ such that $Q[A] \leq r^{2}$. Then

$$
Q[Q[A \mid \xi]>r] \leq r
$$

Proof of Lemma 5.5. This follows directly from

$$
r^{2} \geq Q[A] \geq \int_{\{Q[A \mid \xi]>r\}} Q[A \mid \xi] d Q \geq r Q[Q[A \mid \xi]>r] .
$$

Lemma 5.6 For some constant $c_{14}>0$ it holds:

$$
\tilde{P}[\xi \notin \Xi] \leq e^{-c_{14} n_{0}} .
$$

Proof of Lemma 5.6. Using the bound (3.16), Lemma 5.5 for $Q=\tilde{P}$, the fact $\tilde{P}[\cdot \mid \xi]=$ $P[\cdot \mid \xi]$, and the definition (5.21) of $\Xi_{\mathrm{I}}$, we obtain for a sufficiently small constant $c_{14}>0$

$$
\tilde{P}\left[\xi \notin \Xi_{\mathrm{I}}\right] \leq e^{-c_{4} n_{0} / 2} \leq \frac{e^{-c_{14} n_{0}}}{3} ;
$$


recall that $n_{0}$ was chosen large enough, see Subsection 2.1. As a consequence of the bounds (3.17) and (3.19) we know

$$
\begin{aligned}
& P\left[\left(E_{\mathrm{stop}, T^{m+1}}^{m+1}\right)^{\mathrm{c}} \cap E^{m} \cap\left\{P\left[E^{m} \mid \xi\right] \geq \frac{1}{2}\right\}\right] \leq e^{-n_{m+1}}, \\
& P\left[\left(E^{m}\right)^{\mathrm{c}} \cap E_{\mathrm{stop}, T^{m}}^{m}\right] \leq c_{5} e^{-c_{6} n_{m}} .
\end{aligned}
$$

We obtain by the bound (5.29), Lemmas 5.3 and 5.5 with $Q=P$, and (5.22):

$$
\tilde{P}\left[\xi \notin \Xi_{\mathrm{II}}\right] \leq|\mathcal{C}|^{4 \ln _{0}^{20}+1} P\left[\xi \notin \Xi_{\mathrm{II}}\right] \leq|\mathcal{C}|^{4 l n_{0}^{20}+1} \sum_{m=1}^{\infty} e^{-n_{m+1} / 2} \leq \frac{e^{-c_{14} n_{0}}}{3} .
$$

Here we used again that $n_{0}$ is large, and that $\left(n_{m}\right)_{m \in \mathbb{N}}$ grows fast; see Definition 3.4. The same argument yields, this time using (5.30) and (5.23):

$$
\tilde{P}\left[\xi \notin \Xi_{\mathrm{III}}\right] \leq|\mathcal{C}|^{4 \ln _{0}^{20}+1} P\left[\xi \notin \Xi_{\mathrm{III}}\right] \leq|\mathcal{C}|^{4 \ln _{0}^{20}+1} \sum_{m=1}^{\infty} c_{5}^{1 / 2} e^{-c_{6} n_{m} / 2} \leq \frac{e^{-c_{14} n_{0}}}{3} .
$$

The combination of (5.28), (5.31), (5.32), and (5.24) proves Lemma 5.6.

Lemma 5.7 For all $\xi \in \Xi$ and all $m \in \mathbb{N}$ the following holds for some constants $c_{15}>0$, $c_{16}>0$ :

$$
P\left[E^{m} \mid \xi\right] \geq 1-\sum_{k=0}^{m} c_{16} e^{-c_{15} n_{k}} \geq \frac{1}{2}
$$

and

$$
P\left[E^{m} \backslash E^{m+1} \mid \xi\right] \leq c_{16} e^{-c_{15} n_{m+1}} .
$$

Proof of Lemma 5.7. Let $\xi \in \Xi$. We prove (5.33) and (5.34) simultaneously by induction over $m$ : For $m=1$ we obtain, since $\xi \in \Xi_{\mathrm{I}}$ and $\xi \in \Xi_{\mathrm{III}}$; see (5.21) and (5.23):

$$
\begin{aligned}
P\left[E^{1} \mid \xi\right] & \geq P\left[E_{\text {stop }, T^{1}}^{1} \mid \xi\right]-P\left[\left(E^{1}\right)^{\mathrm{c}} \cap E_{\text {stop }, T^{1}}^{1} \mid \xi\right] \\
& \geq 1-e^{-c_{4} n_{0} / 2}-c_{5}^{1 / 2} e^{-c_{6} n_{1} / 2} \geq 1-\sum_{m=0}^{1} c_{16} e^{-c_{15} n_{m}} \geq \frac{1}{2}
\end{aligned}
$$

for some constants $c_{16}, c_{15}$; recall that $n_{1} \geq n_{0}$ and $n_{0}$ is large enough by Subsection 2.1. Thus (5.33) holds for $m=1$. Let $m \geq 1$. Using $\xi \in \Xi_{\mathrm{II}}$, (5.22), and our induction hypothesis (5.33), we see $P\left[\left(E_{\mathrm{stop}, T^{m+1}}^{m+1}\right)^{\mathrm{c}} \cap E^{m} \mid \xi\right] \leq e^{-n_{m+1} / 2}$. Hence we obtain (5.34), using $\xi \in \Xi_{\text {III }}$ and (5.23):

$$
\begin{aligned}
P\left[E^{m} \backslash E^{m+1} \mid \xi\right] & \leq P\left[\left(E^{m+1}\right)^{\mathrm{c}} \cap E_{\text {stop }, T^{m+1}}^{m+1} \mid \xi\right]+P\left[\left(E_{\text {stop }, T^{m+1}}^{m+1}\right)^{\mathrm{c}} \cap E^{m} \mid \xi\right] \\
& \leq c_{5}^{1 / 2} e^{-c_{6} n_{m+1} / 2}+e^{-n_{m+1} / 2} \leq c_{16} e^{-c_{15} n_{m+1}} .
\end{aligned}
$$

Consequently we get, using our induction hypothesis (5.33) again:

$$
P\left[E^{m+1} \mid \xi\right] \geq P\left[E^{m} \mid \xi\right]-P\left[E^{m} \backslash E^{m+1} \mid \xi\right] \geq 1-\sum_{k=0}^{m+1} c_{16} e^{-c_{15} n_{k}} \geq \frac{1}{2}
$$

this completes our induction step. 
Lemma 5.8 For some constant $c_{17}>0$ and for all $\xi \in \Xi$,

$$
\tilde{P}\left[\bigcup_{m=1}^{\infty}\left(E^{m}\right)^{\mathrm{c}} \mid \xi\right] \leq e^{-c_{17} n_{0}} .
$$

Proof of Lemma 5.8. By Lemma 5.7 we have for $\xi \in \Xi$ :

$$
P\left[\bigcup_{m=1}^{k}\left(E^{m}\right)^{\mathrm{c}} \mid \xi\right] \leq P\left[\left(E^{1}\right)^{\mathrm{c}} \mid \xi\right]+\sum_{m=1}^{k} P\left[E^{m} \backslash E^{m+1} \mid \xi\right] \leq \sum_{m=0}^{k} c_{16} e^{-c_{15} n_{m}} \leq e^{-c_{17} n_{0}}
$$

where $c_{17}<c_{15}$ is a small positive constant; recall that $n_{0}$ is large. In the limit as $k \rightarrow \infty$, this yields the result (5.38).

Proof of Theorem 3.12. Using Lemma 5.6 we have

$$
\begin{aligned}
\tilde{P}\left[\bigcup_{m=1}^{\infty}\left(E^{m}\right)^{\mathrm{c}}\right] & \leq \tilde{P}[\xi \notin \Xi]+\tilde{P}\left[\{\xi \in \Xi\} \cap \bigcup_{m=1}^{\infty}\left(E^{m}\right)^{\mathrm{c}}\right] \\
& \leq e^{-c_{14} n_{0}}+\int_{\{\xi \in \Xi\}} \tilde{P}\left[\bigcup_{m=1}^{\infty}\left(E^{m}\right)^{\mathrm{c}} \mid \xi\right] \mathrm{d} \tilde{P} \\
& \leq e^{-c_{14} n_{0}}+\sup _{\xi \in \Xi} \tilde{P}\left[\bigcup_{m=1}^{\infty}\left(E^{m}\right)^{\mathrm{c}} \mid \xi\right] .
\end{aligned}
$$

We bound the argument of the last supremum, using Lemma 5.8:

$$
\tilde{P}\left[\bigcup_{m=1}^{\infty}\left(E^{m}\right)^{\mathrm{c}} \mid \xi\right]=P\left[\bigcup_{m=1}^{\infty}\left(E^{m}\right)^{\mathrm{c}} \mid \xi\right] \leq e^{-c_{17} n_{0}}
$$

The combination of (5.40) and (5.41) yields, since $n_{0}$ is large (by Subsection 2.1):

$$
\tilde{P}\left[\bigcup_{m=1}^{\infty}\left(E^{m}\right)^{\mathrm{c}}\right] \leq e^{-c_{14} n_{0}}+e^{-c_{17} n_{0}} \leq \frac{1}{3} .
$$

\section{Playing Puzzle: Correctness of the Algorithm $\operatorname{Alg}^{n}$}

In this section we prove Theorem 3.10 by showing that the Algorithm $\mathrm{Alg}^{n}$ defined in Definition 4.5 fulfills the specification described by this theorem: Let $n=n_{m}, m \in \mathbb{N}$. A remark concerning notation: Events defined in this section are labeled with an upper index $n$, not $m$, since the "hierarchy level" $m$ plays no role here, in contrast to the "Skeleton" section. Only events that also occur in the "Skeleton" section keep their old index $m$. Hopefully, this should not cause any confusion.

Let $\tau=\left(\tau_{k}\right)_{k \in \mathbb{N}}$ denote a fixed vector of $\mathcal{G}$-adapted stopping times with values in $\left[0,2^{12 \alpha n}\right]$. We abbreviate Input $:=\left(\tau(\chi), \chi\left\lceil\left[0,2 \cdot 2^{12 \alpha n}[)\right.\right.\right.$. 
Definition 6.1 We define the following events:

$$
\begin{aligned}
& E_{\text {xi does it }}^{n}:=\left\{\xi\left[\left[-5 \cdot 2^{n}, 5 \cdot 2^{n}\right] \in \text { SolutionPieces }^{n} \text { (Input) }\right\},\right. \\
& E_{\text {all pieces ok }}^{n}:=\left\{\begin{array}{l}
\forall w \in \text { SolutionPieces } \\
\\
\xi\left[[ - 2 ^ { n } , 2 ^ { n } ] \preccurlyeq w \preccurlyeq \xi \left\lceil\left[-9 \cdot 2^{n}, 9 \cdot 2^{n}\right]\right.\right.
\end{array}\right\} .
\end{aligned}
$$

Lemma 6.2

$$
E_{\text {xi does it }}^{n} \cap E_{\text {all pieces ok }}^{n} \subseteq E_{\text {reconst, } \operatorname{Alg}^{n}(\tau, \cdot)}^{m}
$$

Proof of Lemma 6.2. When the event $E_{\text {xidoes it }}^{n}$ holds, then the set SolutionPieces ${ }^{n}$ (Input) is not empty. Thus $\operatorname{Alg}^{n}$ (Input) is the lexicographically smallest element of SolutionPieces ${ }^{n}$ (Input). When the event $E_{\text {all pieces ok }}^{n}$ also holds, then $\xi\left[\left[-2^{n}, 2^{n}\right] \preccurlyeq \operatorname{Alg}^{n}(\operatorname{Input}) \preccurlyeq \xi\left\lceil\left[-9 \cdot 2^{n}, 9 \cdot 2^{n}\right]\right.\right.$.

Here is the main theorem of this section; it states that the events $E_{\text {xidoes it }}^{n}$ and $E_{\text {all pieces ok }}^{n}$ occur very probably whenever the stopping times $\tau$ fulfill their task specified by $E_{\mathrm{stop}, \tau}^{m}$ :

Theorem 6.3 For some constant $c_{6}>0, c_{5}>0$ :

$$
P\left[E_{\text {stop }, \tau}^{m} \backslash\left(E_{\text {xi does it }}^{n} \cap E_{\text {all pieces ok }}^{n}\right)\right] \leq c_{5} e^{-c_{6} n} .
$$

This theorem is proven the following three subsections. We split the proof into a purely combinatoric part and a probabilistic part. The combinatoric part (Subsection 6.1 below for $E_{\text {xi does it }}^{n}$ and Subsection 6.2 below for $E_{\text {all pieces ok }}^{n}$ ) shows that whenever some more "basic" events (named $B_{\ldots}^{n}$ below, where "..." stands for a varying label) and $E_{\mathrm{stop}, \tau}^{m}$ occur, then the events $E_{\text {xi doesit }}^{n}$ and $E_{\text {all pieces ok }}^{n}$ occur, too. In the probabilistic part (Subsection 6.3 below) we show that these basic events $B_{\ldots}^{n}$ are highly probable, at least when $E_{\mathrm{stop}, \tau}^{m}$ occurs.

The Proof of Theorem 3.10 is an immediate consequence of Lemma 6.2 and Theorem 6.3.

\subsection{Combinatorics concerning $E_{\text {xidoes it }}^{n}$}

In this subsection, we show that a piece of $\xi$ centered at the origin passes all the tests specified by the Filter $_{i}$, provided some basic events $B_{\ldots}^{n}$ (specified below) hold.

Definition 6.4 For $n \in \mathbb{N}$ we define the following events:

$$
\begin{gathered}
B_{\mathrm{sig} \mathrm{rl}}^{n}:=\left\{\begin{array}{l}
\text { For every right ladder path } \pi \in\left[-2 \cdot l 2^{2 n}, 2 \cdot l 2^{2 n}\right]^{\left[0, c_{1} n / 2[\right.} \text { and for } \\
\text { every admissible piece of path } \pi^{\prime} \in \operatorname{AdPath}\left(2 \cdot l 2^{2 n}, c_{1} n / 2\right): \\
\text { If } \xi \circ \pi=\xi \circ \pi^{\prime}, \text { then } \pi\left(c_{1} n / 2-1\right) \geq \pi^{\prime}\left(c_{1} n / 2-1\right) .
\end{array}\right\}, \\
B_{\mathrm{sig} \mathrm{rr}}^{n}:=\left\{\begin{array}{l}
\text { For every right ladder path } \pi \in\left[-2 \cdot l 2^{2 n}, 2 \cdot l 2^{2 n}\right]^{\left[0, c_{1} n / 2[\right.} \text { and for } \\
\text { every admissible piece of path } \pi^{\prime} \in \operatorname{AdPath}\left(2 \cdot l 2^{2 n}, c_{1} n / 2\right): \\
\text { If } \xi \circ \pi=\xi \circ \pi^{\prime}, \text { then } \pi(0) \leq \pi^{\prime}(0) .
\end{array}\right\} .
\end{gathered}
$$

Let $B_{\mathrm{sig} \mathrm{Il}}^{n}$ and $B_{\mathrm{sig} \mathrm{Ir}}^{n}$ be defined just as $B_{\mathrm{sig} \mathrm{rl}}^{n}$ and $B_{\mathrm{sig} \mathrm{rr}}^{n}$ with "right ladder path" replaced by "left ladder path" and with " $\leq$ " and " $\geq$ " exchanged in (6.5) and (6.6). We set

$$
\begin{aligned}
B_{\text {signals }}^{n} & :=B_{\text {sig } r l}^{n} \cap B_{\text {sig rr }}^{n} \cap B_{\text {sig } l 1}^{n} \cap B_{\text {sig lr }}^{n}, \\
E_{\text {signals II }}^{n}: & =\left\{\begin{array}{l}
\text { For every ladder path } \pi \in\left[-2 \cdot l 2^{2 n}, 2 \cdot l 2^{2 n}\right]^{\left[0, c_{1} n[\right.} \text { and for every } \\
\text { admissible piece of path } \pi^{\prime} \in \operatorname{AdPath}\left(2 \cdot l 2^{2 n}, c_{1} n\right): \\
\text { If } \xi \circ \pi=\xi \circ \pi^{\prime}, \text { then } \pi\left(c_{1} n / 2\right)=\pi^{\prime}\left(c_{1} n / 2\right) .
\end{array}\right\} .
\end{aligned}
$$


Lemma 6.5 $B_{\text {signals }}^{n} \subseteq E_{\text {signals II }}^{n}$

Proof of Lemma 6.5. Assume that the event $B_{\text {signals }}^{n}$ occurs. Let $\pi \in\left[-2 \cdot l 2^{2 n}, 2 \cdot l 2^{2 n}\right]^{\left[0, c_{1} n[\right.}$ be a right ladder path and $\pi^{\prime} \in \operatorname{AdPath}\left(2 \cdot l 2^{2 n}, c_{1} n\right)$. Assume that $\xi \circ \pi=\xi \circ \pi^{\prime}$ holds. Looking at the first half of $\pi$ and $\pi^{\prime}$ only (with the first points $(0, \pi(0)),\left(0, \pi^{\prime}(0)\right)$ dropped), we see $\pi\left(c_{1} n / 2\right) \geq \pi^{\prime}\left(c_{1} n / 2\right)$, since $B_{\mathrm{sig} \mathrm{rl}}^{n}$ holds. Similarly, looking at the second half of $\pi$ and $\pi^{\prime}$ only, we infer $\pi\left(c_{1} n / 2\right) \leq \pi^{\prime}\left(c_{1} n / 2\right)$, since $B_{\text {sig rr }}^{n}$ holds. Therefore $\pi\left(c_{1} n / 2\right)$ and $\pi^{\prime}\left(c_{1} n / 2\right)$ coincide. The case of left ladder paths is treated similarly. This shows that $E_{\text {signals II }}^{n}$ holds.

Definition 6.6 By definition, the event $B_{\text {all paths, } \tau}^{n}$ occurs if and only if the following holds: every admissible piece of path $R \in\left[-12 \cdot 2^{n}, 12 \cdot 2^{n}\right]^{\left[0,3 c_{1} n[\right.}$ occurs in the random walk $S$ with start at most $2^{2 n}$ time steps after some stopping time $\tau(k), k<2^{\alpha n}$. More formally:

$$
B_{\text {all paths }, \tau}^{n}:=\left\{\begin{array}{l}
\forall R \in \operatorname{AdPaths}\left(12 \cdot 2^{n}, 3 c_{1} n\right) \exists k \in\left[0,2^{\alpha n}\left[\exists j \in\left[0,2^{2 n}-3 c_{1} n\right]:\right.\right. \\
\operatorname{TimeShift}^{\tau(k)+j}(R) \subseteq S
\end{array}\right\} .
$$

The following auxiliary lemma helps us to show below that the true scenery $\xi$ passes the test Filter ${ }_{1}$. Roughly speaking, it tells us that sufficiently many ladder words occur in the puzzle. This is important, since playing our puzzle game would lead to a failure if pieces were missing.

Lemma 6.7 Assume that the event $B_{\text {all paths, } \tau}^{n} \cap B_{\text {signals }}^{n} \cap E_{\text {stop }, \tau}^{m}$ holds. Let $I \subseteq\left[-6 \cdot 2^{n}, 6 \cdot 2^{n}\right]$ be a right (or left) ladder interval with $|I|=3 c_{1} n$, and let $w_{1}, w_{2}, w_{3} \in \mathcal{C}^{c_{1} n}$ with $\left(\xi\lceil I)_{\rightarrow}=w_{1} w_{2} w_{3}\right.$ (or $\left(\xi\lceil I)_{\leftarrow}=w_{1} w_{2} w_{3}\right.$ in the case of a left ladder interval). Then $\left(w_{1}, w_{2}, w_{3}\right) \in$ Puzzle $\mathrm{I}_{\mathrm{I}}^{n}$ (Input).

Proof of Lemma 6.7. Assume that $I$ is a right ladder interval; the case of left ladder intervals can be treated in the same way by exchanging "left" and "right". Let $I=I_{1} \cup I_{2} \cup I_{3}$, where $I_{1}$, $I_{2}$, and $I_{3}$ denote the left, middle, and right third of $I$, respectively; thus $\left(\xi\left\lceil I_{i}\right)_{\rightarrow}=w_{i}, i=1,2,3\right.$. Since the event $B_{\text {all paths, } \tau}^{n}$ holds, the straight path which steps through the elements of $I$ from the left to the right in $3 c_{1} n$ steps is realized at least once by the random walk $(S(t))_{t \geq 0}$ within time $2^{2 n}$ of a stopping time $\tau(k), k<2^{\alpha n}$. Observing $\xi$ along such a straight path generates the word $w_{1} w_{2} w_{3}$. Thus

$$
\left(w_{1}, w_{2}, w_{3}\right) \in \text { PrePuzzle }^{n} \text { (Input). }
$$

Let $w_{2}^{\prime}$ be such that $\left(w_{1}, w_{2}^{\prime}, w_{3}\right) \in$ PrePuzzle $^{n}$ (Input). In order to prove the claim $\left(w_{1}, w_{2}, w_{3}\right) \in$ Puzzle ${ }_{\mathrm{I}}^{n}$ (Input) it remains to show: $w_{2}=w_{2}^{\prime}$. When the event $E_{\mathrm{stop}, \tau}^{m}$ holds, the stopping times of $\tau(k), k<2^{\alpha n}$, all stop the random walk $(S(t))_{t \geq 0}$ somewhere in the interval $\left[-2^{n}, 2^{n}\right]$. Within time $2^{2 n}$ the random walk moves at most a distance $l 2^{2 n}$. Because of $w_{1} w_{2}^{\prime} w_{3} \in$ PrePuzzle $^{n}$ (Input), the word $w_{1} w_{2}^{\prime} w_{3}$ occurs somewhere in the observations at most $2^{2 n}$ time steps after a stopping time $\tau(k), k<2^{\alpha n}$. Within time $2^{2 n}$ after a stopping time, the random walk cannot be further away from the origin than $l 2^{2 n}+2^{n} \leq 2 \cdot l 2^{2 n}$, since the event $E_{\mathrm{stop}, \tau}^{m}$ holds. Thus there exists an admissible piece of path $R^{\prime}:\left[0,3 c_{1} n\left[\rightarrow\left[-2 \cdot l 2^{2 n}, 2 \cdot l 2^{2 n}\right]\right.\right.$ such that $\xi \circ R^{\prime}=w_{1} w_{2}^{\prime} w_{3}$. Let $R:\left[0,3 c_{1} n\left[\rightarrow I \subseteq\left[-2 \cdot l 2^{2 n}, 2 \cdot l 2^{2 n}\right]\right.\right.$ denote the right ladder path which passes through $I$ from the left to the right. We know $\xi \circ R^{\prime}\left\lceil\left[0, c_{1} n\left[=\xi \circ R\left\lceil\left[0, c_{1} n\left[=w_{1}\right.\right.\right.\right.\right.\right.$ and $\left(\xi \circ R^{\prime}\left\lceil\left[2 c_{1} n, 3 c_{1} n[)_{\rightarrow}=\left(\xi \circ R\left\lceil\left[2 c_{1} n, 3 c_{1} n[)_{\rightarrow}=w_{3}\right.\right.\right.\right.\right.\right.$. Furthermore, the event $E_{\text {signals II }}^{n} \supseteq B_{\text {signals }}^{n}$ holds; see Lemma 6.5. Abbreviating $x:=c_{1} n / 2$ and $y:=5 c_{1} n / 2$, this implies $R^{\prime}(x)=R(x)$ and $R^{\prime}(y)=R(y)$. But $R\left\lceil[x, y]\right.$ is a right ladder path; thus $R^{\prime}\lceil[x, y]$ must be the same right ladder path, since only right ladder paths can travel equally fast to the right as $R$ does. Hence $w_{2}=\left(\xi \circ R\left\lceil\left[c_{1} n, 2 c_{1} n[)_{\rightarrow}=\left(\xi \circ R^{\prime}\left\lceil\left[c_{1} n, 2 c_{1} n[)_{\rightarrow}=w_{2}^{\prime}\right.\right.\right.\right.\right.\right.$. This finishes the proof of Lemma 6.7. 
Corollary 6.8 If the event $B_{\text {all paths, } \tau}^{n} \cap B_{\text {signals }}^{n} \cap E_{\text {stop }, \tau}^{m}$ holds, then $\xi\left\lceil\left[-5 \cdot 2^{n}, 5 \cdot 2^{n}\right] \in\right.$ Filter ${ }_{1}^{n}$ (Input).

Proof of Corollary 6.8. Assume that $B_{\text {all paths, } \tau}^{n} \cap B_{\text {signals }}^{n} \cap E_{\text {stop }, \tau}^{m}$ holds, and let $I_{2} \subseteq$ $\left[-5 \cdot 2^{n}, 5 \cdot 2^{n}\right],\left|I_{2}\right|=c_{1} n$, be a right ladder interval. Set $I_{1}:=I_{2}-c_{1} n l_{\rightarrow}$ and $I_{3}:=I_{2}+c_{1} n l_{\rightarrow}$; these are right ladder intervals adjacent to the left and to the right of $I_{2}$, respectively. Thus $I:=I_{1} \cup I_{2} \cup I_{3}$ is a right ladder interval, $|I|=3 c_{1} n$. Since $n \geq n_{0}$ and $n_{0}$ is large enough, we obtain $I \subseteq\left[-6 \cdot 2^{n}, 6 \cdot 2^{n}\right]$. We set $w_{i}:=\left(\xi\left\lceil I_{i}\right)_{\rightarrow}, i=1,2,3\right.$. We have $\left(w_{1}, w_{2}, w_{3}\right) \in$ Puzzle $_{\mathrm{I}}^{n}$ (Input) by Lemma 6.7 ; thus $w_{2} \in$ Puzzle $e_{\text {II }}^{n}$ (Input). This finishes the proof of Corollary 6.8.

The following definitions are analogous to the definition of Filter ${ }_{2}^{n}$ and Filter ${ }_{3}^{n}$, with the "reconstructed candidate" $w$ replaced by the true scenery $\xi$, and with the domain $\left[-5 \cdot 2^{n}, 5 \cdot 2^{n}\right]$ replaced by the larger domain $\left[-9 \cdot 2^{n}, 9 \cdot 2^{n}\right]$. We insert the corresponding statements for left ladder intervals, too; this turns out to be useful only in the next subsection.

\section{Definition 6.9}

$$
\begin{aligned}
& E_{\text {neighbor I }}^{n}:= \\
& \left\{\begin{array}{l}
\text { For all right ladder intervals } I, J \subseteq\left[-9 \cdot 2^{n}, 9 \cdot 2^{n}\right]: \quad \text { if } I \triangleright_{n} J, \text { then } \\
\left(\left(\xi\lceil I)_{\rightarrow},\left(\xi\lceil J)_{\rightarrow}\right) \in \text { Neighbors }{ }^{n}(\tau, \eta) .\right.\right. \\
\text { For all left ladder intervals } I, J \subseteq\left[-9 \cdot 2^{n}, 9 \cdot 2^{n}\right]: \\
\left(\left(\xi\lceil I)_{\leftarrow},\left(\xi\lceil J)_{\leftarrow}\right) \in \text { Neighbors }^{n}(\tau, \eta) .\right.\right.
\end{array}\right. \\
& E_{\text {neighbor II }}^{n}:= \\
& \text { (For all right ladder intervals } I, J \subseteq\left[-9 \cdot 2^{n}, 9 \cdot 2^{n}\right],|I|=|J|=c_{1} n \text { : } \\
& \text { if }\left(\left(\xi\lceil I)_{\rightarrow},\left(\xi\lceil J)_{\rightarrow}\right) \in \text { Neighbors }^{n}(\tau, \eta) \text {, then there is } q \in \mathbb{N}\right.\right. \text { such that } \\
& \left\{\triangleright_{n} J+q l_{\rightarrow}\right. \text {. } \\
& \text { For all left ladder intervals } \left.I, J \subseteq\left[-9 \cdot 2^{n}, 9 \cdot 2^{n}\right],|I|=|J|=c_{1} n \text { : if }\right\} \text {. } \\
& \left(\left(\xi\lceil I)_{\leftarrow},\left(\xi\lceil J)_{\leftarrow}\right) \in \text { Neighbors }^{n}(\tau, \eta) \text {, then there is } q \in \mathbb{N} \text { such that } I \triangleleft_{n} J-q l_{\leftarrow}\right.\right. \text {. }
\end{aligned}
$$

Lemma 6.10 If the event $B_{\text {all paths, } \tau}^{n}$ holds, then the event $E_{\text {neighbor I }}^{n}$ holds too, and consequently $\xi\left\lceil\left[-5 \cdot 2^{n}, 5 \cdot 2^{n}\right] \in\right.$ Filter $_{2}^{n}$ (Input).

Proof of Lemma 6.10. Assume that the event $B_{\text {all paths }, \tau}^{n}$ holds. We treat only the case of right ladder intervals; the case of left ladder intervals can be treated in the same way by exchanging right with left, $\rightarrow$ with $\leftarrow$, and $\triangleright_{n}$ with $\triangleleft_{n}$.

Let $I, J \subseteq\left[-9 \cdot 2^{n}, 9 \cdot 2^{n}\right]$ be right ladder intervals such that $I \triangleright_{n} J$. We need to prove $\left(\left(\xi\lceil I)_{\rightarrow},\left(\xi\lceil J)_{\rightarrow}\right) \in\right.\right.$ Neighbors $^{n}$ (Input). Let $i_{l}:=\min I, i_{r}:=\max I, j_{l}:=\min J$, and $j_{r}:=$ $\max J$. Since $I \triangleright_{n} J$, there exists an admissible piece of path consisting of $h+1=l|\mathcal{M}|+1$ points starting in $i_{r}$ and ending in $j_{l}$. Since $I \triangleright_{n} J$ we have $|I|,|J|=c_{1} n$. Thus there exists an admissible piece of path $R:\left[0,2 c_{1} n+h-1\left[\rightarrow\left[i_{l}, j_{r}\right]\right.\right.$ starting at $i_{l}$ and ending in $j_{r}$; furthermore we can require that $R\left\lceil\left[0, c_{1} n\left[\right.\right.\right.$ and $R\left\lceil\left(c_{1} n+h-1+\left[0, c_{1} n[)\right.\right.\right.$ are right ladder paths. Set $w_{1}=(\xi\lceil I) \rightarrow$ and $w_{2}=\left(\xi\lceil J)_{\rightarrow}\right.$; then $\xi \circ R=w_{1} w w_{2}$ where $w \in \mathcal{C}^{h-1}$. Since $n \geq n_{0}$ holds and $n_{0}$ is large enough, we have $h \leq c_{1} n$. Thus the piece of path $\mathrm{R}$ has length shorter than or equal to $3 c_{1} n$. The range $\operatorname{rng}(R)$ of $R$ fulfills $\operatorname{rng}(R) \subseteq\left[-10 \cdot 2^{n}, 10 \cdot 2^{n}\right]$, and since $B_{\text {all paths }, \tau}^{n}$ holds, the random walk $(S(t))_{t \geq 0}$ "follows the path" $R$ at least once within time $2^{2 n}$ after a stopping time of $\tau$. In other words, there exists $k \in\left[0,2^{\alpha n}\right.$ [ and $j \in\left[0,2^{2 n}-2 c_{1} n-h+1\right]$ such that for all $i \in\left[0,2 c_{1} n+h-1[\right.$ we have $S(\tau(k)+j+i)=R(i)$. Thus we get $\xi \circ S\left\lceil\left(\tau(k)+j+\left[0,2 c_{1} n+h-1[) \equiv w_{1} w w_{2}\right.\right.\right.$. This implies that $\left(w_{1}, w_{2}\right) \in$ Neighbors $^{n}($ Input $)$ and thus $\left(\left(\xi\lceil I)_{\rightarrow},\left(\xi\lceil J)_{\rightarrow}\right) \in\right.\right.$ Neighbors $^{n}$ (Input). 
The following elementary number theoretic lemma serves to replace admissible pieces of path with more than $h$ steps by admissible pieces of path with $h$ steps, up to a sequence of maximal steps in one direction:

Lemma 6.11 Let $s=\left(s_{j}\right)_{j=1, \ldots, K} \in \mathcal{M}^{K}, K \in \mathbb{N}$. Then there is $\left(r_{j}\right)_{j=1, \ldots, h} \in \mathcal{M}^{h}$ with

$$
\sum_{j=1}^{h} r_{j}+(K-h) l_{\rightarrow}-\sum_{j=1}^{K} s_{j} \in l_{\rightarrow} \mathbb{N} .
$$

Similarly, there is $\left(r_{j}^{\prime}\right)_{j=1, \ldots, h} \in \mathcal{M}^{h}$ with

$$
\sum_{j=1}^{h} r_{j}^{\prime}-(K-h) l_{\leftarrow}-\sum_{j=1}^{K} s_{j} \in-l_{\leftarrow} \mathbb{N} .
$$

Proof. In order to treat (6.13) and (6.14) simultaneously, let $l_{\leftrightarrow}$ denote either $l_{\rightarrow}$ or $-l_{\leftarrow}$. For $a \in \mathcal{M}$ let $n_{a}$ denote the number of $j=1, \ldots, K$ such that $s_{j}=a$. Let $n_{a}^{\prime} \in\left[0,\left|l_{\leftrightarrow}\right|\left[\cap\left(n_{a}+l_{\leftrightarrow} \mathbb{Z}\right)\right.\right.$ denote the remainder of $n_{a}$ modulo $l_{\leftrightarrow}$. Then $\sum_{a \in \mathcal{M}} n_{a}^{\prime} \leq h$. Choose any list $\left(r_{j}\right)_{j=1, \ldots, h} \in \mathcal{M}^{h}$ having $n_{a}^{\prime}$ entries $a$ for every $a \in \mathcal{M} \backslash\left\{l_{\leftrightarrow}\right\}$ and $h-\sum_{a \in \mathcal{M} \backslash\left\{l_{\leftrightarrow}\right\}} n_{a}^{\prime}$ entries $l_{\leftrightarrow}$. Set

$$
q:=\frac{1}{l_{\leftrightarrow}} \sum_{a \in \mathcal{M}}\left(n_{a}-n_{a}^{\prime}\right)\left(l_{\leftrightarrow}-a\right) \in \mathbb{N} ;
$$

note $\left(l_{\leftrightarrow}-a\right) / l_{\leftrightarrow} \geq 0$ and $n_{a}-n_{a}^{\prime} \in\left|l_{\leftrightarrow}\right| \mathbb{N}$. Then

$$
\begin{aligned}
& \sum_{j=1}^{K}\left(l_{\leftrightarrow}-s_{j}\right)=\sum_{a \in \mathcal{M}} n_{a}\left(l_{\leftrightarrow}-a\right) \\
& =q l_{\leftrightarrow}+\sum_{a \in \mathcal{M}} n_{a}^{\prime}\left(l_{\leftrightarrow}-a\right)=q l_{\leftrightarrow}+\sum_{j=1}^{h}\left(l_{\leftrightarrow}-r_{j}\right),
\end{aligned}
$$

which implies the claim (6.13) or (6.14), respectively.

Lemma 6.12 If the event $E_{\text {signals II }}^{n} \cap E_{\text {stop }, \tau}^{m}$ holds, then the event $E_{\text {neighbor II }}^{n}$ holds, too, and consequently $\xi\left[\left[-5 \cdot 2^{n}, 5 \cdot 2^{n}\right] \in\right.$ Filter $_{3}^{n}$ (Input).

Proof. Assume that the events $E_{\text {signals II }}^{n}$ and $E_{\text {stop }, \tau}^{m}$ hold. We treat here the case of right ladder intervals:

Let $I, J \subseteq\left[-9 \cdot 2^{n}, 9 \cdot 2^{n}\right]$ be right ladder intervals with $|I|=|J|=c_{1} n$, and assume $\left(\left(\xi\lceil I)_{\rightarrow},\left(\xi\lceil J)_{\rightarrow}\right) \in\right.\right.$ Neighbors $^{n}$ (Input). We need to show $I \triangleright_{n} J+q l_{\rightarrow}$ for some $q \in \mathbb{N}$.

Using Definition 4.7 of Neighbors ${ }^{n}$ and the abbreviations $w_{1}:=\left(\xi\lceil I)_{\rightarrow}\right.$ and $w_{2}:=\left(\xi\lceil J)_{\rightarrow}\right.$, we see: There is an admissible piece of path $R:\left[0,2 c_{1} n+h-1[\rightarrow \mathbb{Z}\right.$ with the following properties:

- $R$ is realized by the random walk $S$ in during some time interval $D \subseteq \tau(k)+\left[0,2^{2 n}\right]$, $|D|=2 c_{1} n+h-1$, for some $k \in\left[0,2^{\alpha n}\right.$. This means: $R$ equals $S\lceil D$ when time-shifted back to the origin.

- Observing the scenery $\xi$ along $R$ produces $w_{1} w w_{2}$ for some $w \in \mathcal{C}^{h-1}$; i.e.: $\xi \circ R=w_{1} w w_{2}$. 
We know $|\tau(k)| \leq 2^{n}$ since the event $E_{\text {stop }, \tau}^{m}$ holds; thus $R$ takes all its values in $\left[-\left(2^{n}+\right.\right.$ $\left.\left.l 2^{2 n}\right), 2^{n}+l 2^{2 n}\right] \subseteq\left[-2 \cdot l 2^{2 n}, 2 \cdot l 2^{2 n}\right]$, since the random walk cannot travel faster than distance $l$ per step. We examine the first $c_{1} n$ steps of $R: \quad\left(\xi \circ R\left\lceil\left[0, c_{1} n[)_{\rightarrow}=w_{1}=\left(\xi\lceil I)_{\rightarrow}\right.\right.\right.\right.$ implies $R\left(c_{1} n / 2\right)=\min I+c_{1} n l_{\rightarrow} / 2$, since the event $E_{\text {signals II }}^{n}$ holds; note that $x:=\min I+c_{1} n l_{\rightarrow} / 2$ is the point in the middle of a right ladder path walking through $I$. The same argument applies to the last $c_{1} n$ steps of $R:\left(\xi \circ R\left\lceil\left(c_{1} n+h-1+\left[0, c_{1} n[)\right)_{\rightarrow}=w_{2}=\left(\xi\lceil J)_{\rightarrow}\right.\right.\right.\right.$ implies $R\left(3 c_{1} n / 2+h-1\right)=$ $\min J+c_{1} n l_{\rightarrow} / 2=: y ; y$ is the point in the middle of $J$. The path $R$ travels from $x$ to $y$ in $K:=c_{1} n+h-1 \geq h$ steps, using some step sizes $\left(s_{j}\right)_{j=1, \ldots, K} \in \mathcal{M}^{K}$. As a consequence of (6.13) in Lemma 6.11, there is $\left(r_{j}\right)_{j=1, \ldots, h} \in \mathcal{M}^{h}$ with $\sum_{j=1}^{h} r_{j}+(K-h) l_{\rightarrow}-\sum_{j=1}^{K} s_{j}=q l_{\rightarrow}$ for some $q \in \mathbb{N}$. Since $\max I-x=\left(c_{1} n / 2-1\right) l_{\rightarrow}$ and $y-\min J=c_{1} n l_{\rightarrow} / 2$, we obtain $\min J-\max I=y-x-\left(c_{1} n-1\right) l_{\rightarrow}=\sum_{j=1}^{K} s_{j}-\left(c_{1} n-1\right) l_{\rightarrow}=\sum_{j=1}^{h} r_{j}-q l_{\rightarrow}$. This means $I \triangleright_{n}\left(J+q l_{\rightarrow}\right)$, as we wanted to show.

Summarizing, this implies $\xi\left[\left[-5 \cdot 2^{n}, 5 \cdot 2^{n}\right] \in\right.$ Filter $_{3}^{n}$ (Input) and the first statement in the definition of $E_{\text {neighbor II }}^{n}$, which treats right ladder intervals.

The proof for left ladder intervals can be treated analogously. Altogether, we see that the event $E_{\text {neighbor II }}^{n}$ is valid.

Definition 6.13 We define the event

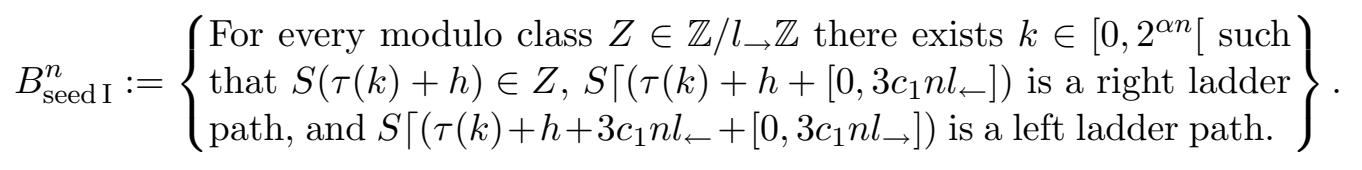

Lemma 6.14 If the events $B_{\text {all paths }, \tau}^{n}, B_{\text {signals }}^{n}, B_{\text {seed I }}^{n}$ and $E_{\mathrm{stop}, \tau}^{m}$ hold, then $\xi\left\lceil\left[-5 \cdot 2^{n}, 5 \cdot 2^{n}\right] \in\right.$ Filter ${ }_{4}^{n}$ (Input).

Proof of Lemma 6.14. Assume that the event $B_{\text {all paths }, \tau}^{n} \cap B_{\text {signals }}^{n} \cap B_{\text {seed I }}^{n} \cap E_{\text {stop }, \tau}^{m}$ holds. Let $Z \in \mathbb{Z} / l_{\rightarrow} \mathbb{Z}$. Since $B_{\text {seed I }}^{n}$ holds, there exists a $k \in\left[0,2^{\alpha n}\left[\operatorname{such}\right.\right.$ that $S(\tau(k)+h) \in Z, R_{1}:=$ $S\left\lceil\left(\tau(k)+h+\left[0,3 c_{1} n l_{\leftarrow}\right]\right)\right.$ is a right ladder path, and $R_{2}:=S\left\lceil\left(\tau(k)+h+3 c_{1} n l_{\leftarrow}+\left[0,3 c_{1} n l_{\rightarrow}\right]\right)\right.$ is a left ladder path. Since $E_{\mathrm{stop}, \tau}^{m}$ holds, we know $S(\tau(k)) \in\left[-2^{n}, 2^{n}\right]$. Thus the random walk $S$ cannot leave the interval $\left[-2 \cdot 2^{n}, 2 \cdot 2^{n}\right]$ during the time interval $\tau(k)+\left[h+3 c_{1} n l_{\leftarrow}+3 c_{1} n l_{\rightarrow}\right]$, since

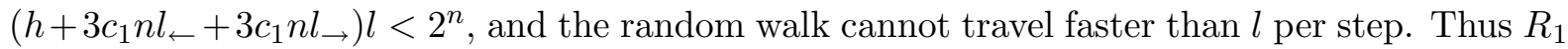
and $R_{2}$ take all their values in $\left[-2 \cdot 2^{n}, 2 \cdot 2^{n}\right.$. Note that the right ladder path $R_{1}$ and the left ladder path walk $R_{2}$ traverse precisely the same interval, $R_{1}$ using step size $l_{\rightarrow}$ to the right, and $R_{2}$ with step size $-l_{\leftarrow}$ back. The same is true when we restrict $R_{1}$ and $R_{2}$ to the smaller time intervals $\left[t_{1}, t_{1}^{\prime}\right]:=\tau(k)+h+c_{1} n l_{\leftarrow}+\left[0, c_{2} n l_{\leftarrow}\right]$ and $\left[t_{2}, t_{2}^{\prime}\right]:=\tau(k)+h+3 c_{1} n l_{\leftarrow}+2 c_{1} n l_{\rightarrow}+\left[-c_{2} n l_{\rightarrow}, 0\right]$, respectively: We have $S\left(t_{1}\right)=S\left(t_{2}^{\prime}\right)=: a$, and $S\left(t_{1}^{\prime}\right)=S\left(t_{2}\right)=: b$, and $S\left\lceil\left[t_{1}, t_{1}^{\prime}\right]\right.$ is a right ladder path: it traverses $[a, b]$ from the left to the right, while on $S\left\lceil\left[t_{2}, t_{2}^{\prime}\right]\right.$ it is a left ladder path; it traverses $[a, b]$ in opposite direction. In particular, reading only every $l_{\leftarrow}$ th letter in $\chi\left\lceil\left[t_{1}, t_{1}^{\prime}\right]\right.$ and only every $l_{\rightarrow}$ th letter in $\chi\left\lceil\left[t_{2}, t_{2}^{\prime}\right]\right.$ yield the same word, only in reversed direction:

$$
\left(\chi \left\lceil\left(\left[t_{1}, t_{1}^{\prime}\right] \cap\left(t_{1}+l_{\leftarrow} \mathbb{Z}\right)\right)_{\rightarrow}=\left(\xi\left\lceil\left([a, b] \cap\left(a+l_{\rightarrow} l_{\leftarrow} \mathbb{Z}\right)\right)\right)_{\rightarrow}=\left(\chi\left\lceil\left[t_{2}, t_{2}^{\prime}\right] \cap\left(t_{1}+l_{\leftarrow} \mathbb{Z}\right)\right)_{\leftarrow .}\right.\right.\right.\right.
$$

We consider the words $u_{1} u_{2} u_{3}:=\chi\left\lceil\left(t_{1}-c_{1} n+\left[0,3 c_{1} n[)\right.\right.\right.$ and $v_{1} v_{2} v_{3}:=\chi\left\lceil\left(t_{2}-c_{1} n+\left[0,3 c_{1} n[)\right.\right.\right.$ with $u_{i}, v_{i} \in \mathcal{C}^{c_{1} n} ;$ note that $t_{1}-c_{1} n+\left[0,3 c_{1} n\left[\subseteq\right.\right.$ domain $\left(R_{1}\right)$ and $t_{2}-c_{1} n+\left[0,3 c_{1} n\left[\subseteq \operatorname{domain}\left(R_{2}\right)\right.\right.$. We get $\left(u_{1}, u_{2}, u_{3}\right),\left(v_{1}, v_{2}, v_{3}\right) \in$ Puzzle $_{\mathrm{I}}^{n}($ Input $)$ by Lemma 6.7 . Hence we obtain $\left(w_{1}, w_{2}, w_{3}\right) \in$ $\operatorname{Seed}_{\mathrm{I}}^{n}$ (Input) by Definition (4.4), since the words $u_{1} u_{2} u_{3}$ and $v_{1} v_{2} v_{3}$ occur in the observations 
sufficiently close to a stopping time $\tau(k)$; more specifically: $t_{1}-c_{1} n, t_{2}-c_{1} n \in \tau(k)+\left[0,7 c_{1} n l\right]$. Consequently $u_{2}, v_{2} \in \operatorname{Seed}_{\mathrm{II}}^{n}$ (Input) by Definition (4.5). Finally we observe

$$
\left(u_{2}\left\lceil\left(\left[0, c_{2} n l_{\leftarrow}\right] \cap l_{\leftarrow} \mathbb{Z}\right)\right)_{\rightarrow}=\left(\xi\left\lceil\left([a, b] \cap\left(a+l_{\rightarrow} l_{\leftarrow} \mathbb{Z}\right)\right)\right)_{\rightarrow}=\left(v_{2}\left\lceil\left(\left[0, c_{2} n l_{\rightarrow}\right] \cap l_{\rightarrow} \mathbb{Z}\right)\right)_{\leftarrow}\right.\right.\right.
$$

by (6.18). Thus we have shown $u_{2} \in \operatorname{Seed}_{\mathrm{III}}^{n}$ (Input), see (4.6). Since $u_{2}=\xi \circ S\left\lceil\left(t_{1}+\left[0, c_{1} n[)\right.\right.\right.$, and since $S\left\lceil\left(t_{1}+\left[0, c_{1} n[)\right.\right.\right.$ is a right ladder path with values in $Z \cap\left[-2 \cdot 2^{n}, 2 \cdot 2^{n}\right]$, this implies $\xi\left\lceil\left[-5 \cdot 2^{n}, 5 \cdot 2^{n}\right] \in\right.$ Filter $_{4}^{n}$ (Input).

Definition 6.15 For $n \in \mathbb{N}$ and and a finite set $\tilde{J} \subset \mathbb{N}$, we define the following event:

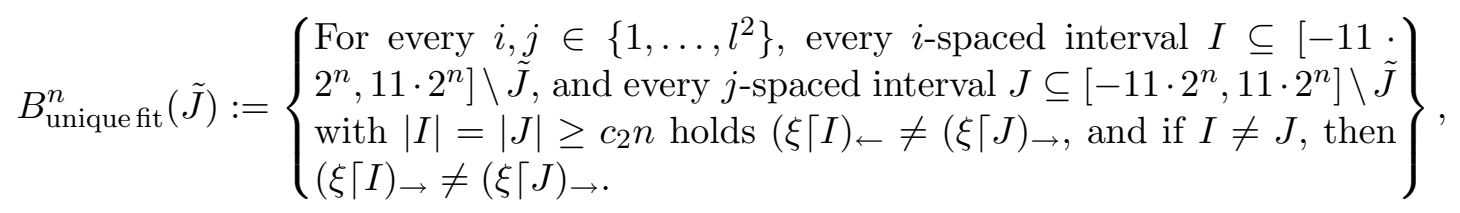

We abbreviate $B_{\text {unique fit }}^{n}:=B_{\text {unique fit }}^{n}(\emptyset)$.

In this section, only the case $\tilde{J}=\emptyset$ is needed. However, in Section 8 below, the case $\tilde{J} \neq \emptyset$ is important, too, due to the presence of a "modified" part of the scenery close to the origin.

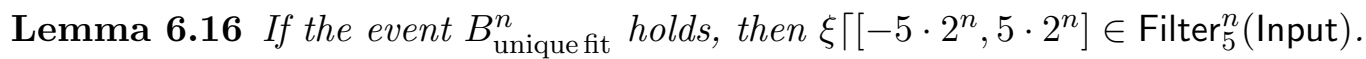

Proof. Using $c_{2} \leq c_{1}$ (see Subsection 2.1), this follows immediately from Definition 6.15 of the event $B_{\text {unique fit }}^{n}$, and of Definition 4.4 of Filter ${ }_{5}^{n}$.

Theorem 6.17 $B_{\text {all paths, } \tau}^{n} \cap B_{\text {signals }}^{n} \cap B_{\text {seed I }}^{n} \cap B_{\text {unique fit }}^{n} \cap E_{\text {stop }, \tau}^{m} \subseteq E_{\text {xi does it }}^{n}$

Proof. We collect the statements of Lemmas/Corollary 6.5, 6.8, 6.10, 6.12, 6.14, and 6.16 in the following list:

$$
\begin{aligned}
& B_{\text {signals }}^{n} \\
& B_{\text {all paths }, \tau}^{n} \cap B_{\text {signals }}^{n} \cap E_{\text {stop }, \tau}^{m} \subseteq\left\{\xi \xi\left\lceil\left[-5 \cdot 2^{n}, 5 \cdot 2^{n}\right] \in \text { Filter }_{1}^{n}(\text { Input })\right\},\right. \\
& B_{\text {all paths, } \tau}^{n} \\
& E_{\text {signals II }}^{n} \cap E_{\text {stop }, \tau}^{m} \subseteq\left\{\xi\left[\left[-5 \cdot 2^{n}, 5 \cdot 2^{n}\right] \in \text { Filter }_{3}^{n} \text { (Input) }\right\},\right. \\
& B_{\text {all paths }, \tau}^{n} \cap B_{\text {signals }}^{n} \cap B_{\text {seed I }}^{n} \cap E_{\text {stop }, \tau}^{m} \subseteq\left\{\xi\left\lceil\left[-5 \cdot 2^{n}, 5 \cdot 2^{n}\right] \in \text { Filter }_{4}^{n} \text { (Input) }\right\},\right. \\
& B_{\text {unique fit }}^{n} \\
& \subseteq\left\{\xi\left\lceil\left[-5 \cdot 2^{n}, 5 \cdot 2^{n}\right] \in \text { Filter }_{5}^{n} \text { (Input) }\right\}\right. \text {. }
\end{aligned}
$$

The theorem is an immediate consequence these statements, using (6.1) and (4.9).

\subsection{Combinatorics concerning $E_{\text {all pieces ok }}^{n}$}

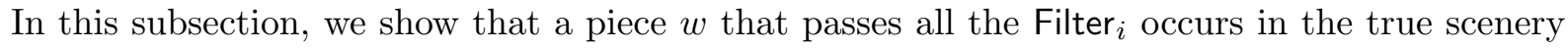
$\xi$ near the origin, provided some "basic" events $B_{\ldots}^{n}$ hold.

Definition 6.18 Given a finite set $\tilde{J} \subset \mathbb{N}$, we define the events

$B_{\text {recogn straight }}^{n}(\tilde{J}):=$

$\left\{\begin{array}{l}\text { For every } R \in \operatorname{AdPaths}\left(11 \cdot 2^{n}, c_{1} n\right) \text { with } R\left(c_{1} n-1\right)-R(0) \notin\left\{\left(c_{1} n-1\right) l_{\rightarrow,}-\left(c_{1} n-1\right) l_{\leftarrow}\right\} \\ \text { there is } \bar{R} \in \operatorname{AdPaths}\left(12 \cdot 2^{n}, c_{1} n\right) \text { such that } R(0)=\bar{R}(0), R\left(c_{1} n-1\right)=\bar{R}\left(c_{1} n-1\right), \text { and } \\ (\bar{R} \text { takes at least one value in } \tilde{J}, \text { or } \xi \circ R \neq \xi \circ \bar{R}) .\end{array}\right.$ 
In the case $\tilde{J}=\emptyset$, we abbreviate $B_{\text {recogn straight }}^{n}:=B_{\text {recogn straight }}^{n}(\emptyset)$.

$$
E_{\text {only ladder }}^{n}:=\left\{\begin{array}{l}
\text { For all }\left(w_{1}, w_{2}, w_{3}\right) \in \text { Puzzle } e_{I}^{n}(\text { Input }) \text { and every admissible piece of } \\
\text { path } R:\left[0,3 c_{1} n\left[\rightarrow\left[-11 \cdot 2^{n}, 11 \cdot 2^{n}\right] \text { with } \xi \circ R=w_{1} w_{2} w_{3}\right. \text { holds: }\right. \\
w_{2} \text { is a ladder word of } \xi\left[\left[-11 \cdot 2^{n}, 11 \cdot 2^{n}\right] .\right.
\end{array}\right\} .
$$

Lemma 6.19 We have

$$
B_{\text {all paths }, \tau}^{n} \cap B_{\text {recogn straight }}^{n} \subseteq E_{\text {only ladder }}^{n} .
$$

Proof of Lemma 6.19. Assume that the event $B_{\text {all paths }, \tau}^{n} \cap B_{\text {recogn straight }}^{n}$ holds. Let $\left(w_{1}, w_{2}, w_{3}\right) \in$ Puzzle $_{\mathrm{I}}^{n}$ (Input), and let $R:\left[0,3 c_{1} n\left[\rightarrow\left[-11 \cdot 2^{n}, 11 \cdot 2^{n}\right]\right.\right.$ be an admissible piece of path with $\xi \circ R=w_{1} w_{2} w_{3}$. We prove by contradiction that the event $E_{\text {only ladder }}^{n}$ holds: Assume $w_{2}$ is not a ladder word of $\xi\left\lceil\left[-11 \cdot 2^{n}, 11 \cdot 2^{n}\right]\right.$. Since $B_{\text {recogn straight }}^{n}$ holds, there exists an admissible piece of path $\bar{R}:\left[c_{1} n, 2 c_{1} n\left[\rightarrow\left[-11 \cdot 2^{n}, 11 \cdot 2^{n}\right]\right.\right.$ such that $R\left(c_{1} n\right)=\bar{R}\left(c_{1} n\right)$ and $R\left(2 c_{1} n-1\right)=\bar{R}\left(2 c_{1} n-1\right)$, but $w_{2} \neq(\xi \circ \bar{R})_{\rightarrow}=: w_{2}^{\prime}$. Let $\breve{R}:\left[0,3 c_{1} n\left[\rightarrow\left[-11 \cdot 2^{n}, 11 \cdot 2^{n}\right]\right.\right.$ be the admissible piece of path which on $\left[c_{1} n, 2 c_{1} n\right.$ [ is equal to $\bar{R}$ and otherwise is equal to $R$. We have $\xi \circ \check{R}=w_{1} w_{2}^{\prime} w_{3}$. Since $B_{\text {all paths, } \tau}^{n}$ holds, too, this implies that the random walk $S$ follows the path of $\check{R}$ within time $2^{2 n}$ from a stopping time of $\tau(k), k<2^{\alpha n}$. The same is valid for $R$, maybe with a different stopping time $\tau\left(k^{\prime}\right)$. In other words: $w_{1} w_{2}^{\prime} w_{3} \in$ PrePuzzle $^{n}$ (Input) and $\left(w_{1}, w_{2}, w_{3}\right) \in$ PrePuzzle $^{n}$ (Input). This implies the contradiction $\left(w_{1}, w_{2}, w_{3}\right) \notin$ Puzzle $_{I}^{n}$ (Input); thus we have proved Lemma 6.19.

Definition 6.20 We define the events

$B_{\text {outside out }}^{n}:=$

$\left\{\begin{array}{l}\text { For every admissible piece of path } \\ R \in\left(\left[-2 \cdot l 2^{2 n}, 2 \cdot l 2^{2 n}\right] \backslash\left[-10 \cdot 2^{n}, 10 \cdot 2^{n}\right]\right)^{\left[0, c_{1} n / 2[\right.}: \xi \circ R \text { is not strongly equivalent } \\ \text { to any ladder word of length } c_{1} n / 2 \text { of } \xi\left\lceil\left[-9 \cdot 2^{n}, 9 \cdot 2^{n}\right] .\right.\end{array}\right.$,

$E_{\text {mod class }}^{n}:=$

$\left\{\begin{array}{l}\text { For all } w \in \text { Filter }_{1}^{n} \text { (Input) and for all right ladder intervals } I \subseteq\left[-2 \cdot 2^{n}, 2 \cdot 2^{n}\right],|I|=c_{1} n: \\ \text { If there is a right ladder interval } J_{r} \subseteq\left[-2 \cdot 2^{n}, 2 \cdot 2^{n}\right] \text { with } w\left\lceil I \equiv \xi\left\lceil J_{r} \text {, then }\right.\right. \\ \xi\left\lceil\left(\left[-2^{n}, 2^{n}\right] \cap\left(J_{r}+l_{\rightarrow} \mathbb{Z}\right)\right) \sqsubseteq w\left\lceil\left(I+l_{\rightarrow} \mathbb{Z}\right) \sqsubseteq \xi\left\lceil\left(\left[-9 \cdot 2^{n}, 9 \cdot 2^{n}\right] \cap\left(J_{r}+l_{\rightarrow} \mathbb{Z}\right)\right) \text {, and }\right.\right.\right. \\ \text { if } l_{\rightarrow}=l_{\leftarrow} \text { and if there is a (left) ladder interval } J_{l} \subseteq\left[-2 \cdot 2^{n}, 2 \cdot 2^{n}\right] \text { with }\left(w\lceil I) \leftrightarrow \equiv \xi\left\lceil J_{l},\right.\right. \\ \text { then } \xi\left\lceil\left(\left[-2^{n}, 2^{n}\right] \cap\left(J_{l}+l \mathbb{Z}\right)\right) \sqsubseteq\left(w\left\lceil(I+l \mathbb{Z}) \leftrightarrow \sqsubseteq \xi\left\lceil\left(\left[-9 \cdot 2^{n}, 9 \cdot 2^{n}\right] \cap\left(J_{l}+l \mathbb{Z}\right)\right) .\right.\right.\right.\right.\end{array}\right\}$.

Informally speaking, the meaning of the event $E_{\text {mod class }}^{n}$ is the following: If a "reconstructed" piece of scenery $w$ contains a correct "seed piece" $w\lceil I$ over a sufficiently long ladder word, then the whole modulo class generated by $I$ is reconstructed correctly. The reconstruction may generate the wrong orientation, but this is only allowed if left ladder intervals and right ladder intervals coincide, and if already the "seed piece" $w\lceil I$ is reversed compared with the true scenery $\xi$.

The next lemma formalizes the intuitive idea of "playing a puzzle game": We start with a seed word as reconstructed piece; then we append successively pieces of our puzzle that match to an ending of the growing reconstructed piece. This procedure continues until the reconstructed piece is large enough. 
Lemma 6.21 We have

$$
B_{\text {outside out }}^{n} \cap B_{\text {unique fit }}^{n} \cap E_{\text {only ladder }}^{n} \cap E_{\text {stop }, \tau}^{m} \subseteq E_{\text {mod class }}^{n}
$$

Proof of Lemma 6.21. Assume that the events on the left hand side of (6.26) hold. We claim that then $E_{\text {mod class }}^{n}$ holds, too. To prove this claim, let $w \in$ Filter $_{1}^{n}$ (Input), and let $I \subseteq$ $\left[-2 \cdot 2^{n}, 2 \cdot 2^{n}\right],|I|=c_{1} n$ be a right ladder interval. Assume that $J \subseteq\left[-2 \cdot 2^{n}, 2 \cdot 2^{n}\right]$ is a ladder interval. We assume one of the following two cases:

A) $J$ is a right ladder interval, and $w\lceil I \equiv \xi\lceil J$;

B) $l_{\rightarrow}=l_{\leftarrow}$ and $(w\lceil I) \leftrightarrow \equiv \xi\lceil J$.

We treat both cases simultaneously as far as possible; in order to unify notation, let $\cdot \sim$ denote the reversion operation $\cdot \leftrightarrow$ in case B and the identity operation in case A. We set $Z:=J+l \rightarrow \mathbb{Z} \in$ $\mathbb{Z} / l_{\rightarrow} \mathbb{Z}$; then it remains to show:

$$
\xi\left\lceil( [ - 2 ^ { n } , 2 ^ { n } ] \cap Z ) \sqsubseteq \left(w \lceil ( I + l _ { \rightarrow } \mathbb { Z } ) ) ^ { \sim } \sqsubseteq \xi \left\lceil\left(\left[-9 \cdot 2^{n}, 9 \cdot 2^{n}\right] \cap Z\right) .\right.\right.\right.
$$

To prove the right hand side of (6.27), we prove by induction over all right ladder intervals $I^{\prime}$ with $I \subseteq I^{\prime} \subseteq\left[-5 \cdot 2^{n}, 5 \cdot 2^{n}\right]$ :

$$
\left(w \lceil I ^ { \prime } ) ^ { \sim } \sqsubseteq \xi \left\lceil\left(\left[-9 \cdot 2^{n}, 9 \cdot 2^{n}\right] \cap Z\right) .\right.\right.
$$

Once we have proven this, the right hand side of (6.27) follows from the special case $I^{\prime}=$ $\left[-5 \cdot 2^{n}, 5 \cdot 2^{n}\right] \cap\left(I+l_{\rightarrow} \mathbb{Z}\right)$.

The induction starts with $I=I^{\prime}$ : in this case (6.28) holds since our assumption A) or B), respectively, implies $\left(w\lceil I)^{\sim} \sqsubseteq \xi\left\lceil\left(\left[-9 \cdot 2^{n}, 9 \cdot 2^{n}\right] \cap Z\right)\right.\right.$. For the induction step, assume that (6.28) holds for some $I^{\prime}$. We enlarge $I^{\prime}$ by a single new point: let $I^{\prime \prime}=I^{\prime} \cup\{i\} \subseteq\left[-5 \cdot 2^{n}, 5 \cdot 2^{n}\right] \cap\left(I+l_{\rightarrow} \mathbb{Z}\right)$ be a right ladder interval, $i \notin I^{\prime}$. Let $I_{i} \subseteq I^{\prime \prime}$ be a right ladder interval with $\left|I_{i}\right|=c_{1} n$ and $i \in I_{i}$. Using $w \in$ Filter $_{1}^{n}$ (Input) we see $w_{2}:=\left(w\left\lceil I_{i}\right)_{\rightarrow} \in \operatorname{Puzzle}_{\mathrm{II}}^{n}\right.$ (Input). Hence there are $w_{1}, w_{3} \in \mathcal{C}^{c_{1} n}$ such that $\left(w_{1}, w_{2}, w_{3}\right) \in$ Puzzle $_{\mathrm{I}}^{n}$ (Input $) \subseteq$ PrePuzzle $^{n}$ (Input). Thus $w_{1} w_{2} w_{3}$ occurs in the observation $\chi$ at most $2^{2 n}$ time steps after a stopping time $\tau(k), k<2^{\alpha n}$; say $w_{1} w_{2} w_{3}$ is read there in $\chi$ while the random walk follows an admissible piece of path $R:\left[0,3 c_{1} n[\rightarrow \mathbb{Z}\right.$; (we shifted the time domain of $R$ back to the origin). Since the event $E_{\mathrm{stop}, \tau}^{m}$ holds, we have $|S(\tau(k))| \leq 2^{n}$. Within time $2^{2 n}$ the random walk cannot travel farther than distance $l 2^{2 n}$; thus $R$ has all its values in $\left[-\left(2^{n}+l 2^{2 n}\right), 2^{n}+l 2^{2 n}\right] \subseteq\left[-2 \cdot l 2^{2 n}, 2 \cdot l 2^{2 n}\right]$. Consider the ladder interval $I_{i}^{\prime}:=I_{i} \backslash\{i\}=I_{i} \cap I^{\prime},\left|I_{i}^{\prime}\right|=c_{1} n-1 \geq c_{1} n / 2$ : the induction hypothesis (6.28) implies $\left(w\left\lceil I_{i}^{\prime}\right)^{\sim} \sqsubseteq\right.$ $\xi\left\lceil\left(\left[-9 \cdot 2^{n}, 9 \cdot 2^{n}\right] \cap Z\right)\right.$; say $\left(w\left\lceil I_{i}^{\prime}\right)^{\sim} \equiv \xi\left\lceil D^{\prime}\right.\right.$ for some right ladder interval $D^{\prime} \subseteq\left[-9 \cdot 2^{n}, 9 \cdot 2^{n}\right] \cap Z$. Furthermore, $w_{2}^{\prime}:=\left(w\left\lceil I_{i}^{\prime}\right)_{\rightarrow}\right.$ is a subword of $w_{2}=\left(w\left\lceil I_{i}\right)_{\rightarrow}\right.$ and thus also a subword of $\xi \circ R$. Hence we see, using that the event $B_{\text {outside out }}^{n}$ holds: $R$ cannot take all of its values outside $\left[-10 \cdot 2^{n}, 10 \cdot 2^{n}\right]$; thus it has all its values in $\left[-10 \cdot 2^{n}-3 c_{1} n l, 10 \cdot 2^{n}+3 c_{1} n l\right] \subseteq\left[-11 \cdot 2^{n}, 11 \cdot 2^{n}\right]$. Since the event $E_{\text {only ladder }}^{n}$ holds, $w_{2}=\left(w\left\lceil I_{i}\right)_{\rightarrow}\right.$ is a ladder word of $\xi\left\lceil\left[-11 \cdot 2^{n}, 11 \cdot 2^{n}\right]\right.$; say $w_{2}=\left(\xi\lceil D)_{\rightarrow}\right.$ for some right ladder interval $D \subseteq\left[-11 \cdot 2^{n}, 11 \cdot 2^{n}\right]$ (we call this "case $\mathrm{A}_{1}$ "), or $w_{2}=\left(\xi\lceil D)_{\leftarrow}\right.$ for some left ladder interval $D \subseteq\left[-11 \cdot 2^{n}, 11 \cdot 2^{n}\right]$ (call this "case $\mathrm{B}_{1}$ "). Thus $w_{2}^{\prime}$ occurs as a (possibly reversed) ladder word

- as a subword of $\left(\xi\lceil D)_{\rightarrow}\right.$ in case $\mathrm{A}_{1}$, or as a subword of $\left(\xi\lceil D)_{\leftarrow}\right.$ in case $\mathrm{B}_{1}$;

- as $w_{2}^{\prime}=\left(\xi\left\lceil D^{\prime}\right)_{\rightarrow}\right.$ in case $\mathrm{A}$, or as $w_{2}^{\prime}=\left(\xi\left\lceil D^{\prime}\right)_{\leftarrow}\right.$ in case B. 
Since the event $B_{\text {unique fit }}^{n}$ holds, this implies $D^{\prime} \subseteq D$, and furthermore the reading directions have to coincide: If case $\mathrm{A}$ holds, then case $\mathrm{A}_{1}$ occurs, and if case $\mathrm{B}$ holds, then case $\mathrm{B}_{1}$ occurs. Let $T: \mathbb{Z} \rightarrow \mathbb{Z}$ denote the translation (case A) or reflection (case B) that transports $w\left\lceil I_{i}\right.$ to $\xi\left\lceil D\right.$. Then $T$ transports $w\left\lceil I_{i}^{\prime}\right.$ to $\xi\left\lceil D^{\prime}\right.$, and thus - using once more that $B_{\text {unique fit }}^{n}$ holds $-T$ is also the map that transports $w\left\lceil I^{\prime}\right.$ to a subpiece of $\xi\left\lceil\left(\left[-9 \cdot 2^{n}, 9 \cdot 2^{n}\right] \cap Z\right)\right.$ according to the induction hypothesis (6.28). Hence $T$ transports $w\left\lceil\left(I_{i} \cup I^{\prime}\right)=w\left\lceil I^{\prime \prime}\right.\right.$ to an equivalent subpiece of $\xi\left\lceil\left[-11 \cdot 2^{n}, 11 \cdot 2^{n}\right]\right.$. To see that $T\left[w\left\lceil I^{\prime \prime}\right]\right.$ is already a subpiece of $\xi\left\lceil\left(\left[-9 \cdot 2^{n}, 9 \cdot 2^{n}\right] \cap Z\right)\right.$, we proceed as follows: $T$ maps the nonempty seed interval $I \subseteq\left[-2 \cdot 2^{n}, 2 \cdot 2^{n}\right]$ to $J \subseteq\left[-2 \cdot 2^{n}, 2 \cdot 2^{n}\right] \cap Z$; thus it has the form $T(z)= \pm z+a$ with $|a| \leq 4 \cdot 2^{n}$. Consequently $T$ maps the domain $\left[-5 \cdot 2^{n}, 5 \cdot 2^{n}\right]$ of $w$ to a subset of $\left[-9 \cdot 2^{n}, 9 \cdot 2^{n}\right]$. This shows $\left(w\left\lceil I^{\prime \prime}\right) \sim \xi\left\lceil\left(\left[-9 \cdot 2^{n}, 9 \cdot 2^{n}\right] \cap Z\right)\right.\right.$, which finishes our induction step and also the proof of the right hand side of the claim (6.27).

To prove the left hand side of $(6.27)$, we observe that $T^{-1}$ maps $\left[-2^{n}, 2^{n}\right]$ to a subset of $\left[-5 \cdot 2^{n}, 5 \cdot 2^{n}\right.$. Since $T$ maps $I$ to $J$, it maps the modulo class $I+l_{\rightarrow} \mathbb{Z}$ to $Z=J+l_{\rightarrow} \mathbb{Z}$; thus $T^{-1}$ maps $\left[-2^{n}, 2^{n}\right] \cap Z$ to a subset of $\left(I+l_{\rightarrow} \mathbb{Z}\right) \cap\left[-5 \cdot 2^{n}, 5 \cdot 2^{n}\right]=\left(I+l_{\rightarrow} \mathbb{Z}\right) \cap \operatorname{domain}(w)$. Since $T^{-1}$ maps a subpiece of $\xi\left\lceil\left(\left[-9 \cdot 2^{n}, 9 \cdot 2^{n}\right] \cap Z\right)\right.$ to $w\left\lceil\left(I+l_{\rightarrow} \mathbb{Z}\right)\right.$, this implies the left hand side of the claim (6.27). This finishes the proof of Lemma 6.21.

Definition 6.22 We define the event

$$
E_{\text {seed II }}^{n}:=\left\{\begin{array}{l}
\text { Every } u \in \operatorname{Seed}_{\mathrm{II}}^{n}(\text { Input }) \text { is a left or right ladder word of } \xi\lceil[-2 \cdot \\
\left.2^{n}, 2 \cdot 2^{n}\right] . \text { If } l_{\rightarrow} \neq l_{\leftarrow}, \text { then every } u \in \operatorname{Seed}_{\mathrm{III}}^{n} \text { (Input) is a right } \\
\text { ladder word of } \xi\left[\left[-2 \cdot 2^{n}, 2 \cdot 2^{n}\right] .\right.
\end{array}\right\}
$$

Lemma 6.23 We have

$$
B_{\text {unique fit }}^{n} \cap B_{\text {signals }}^{n} \cap B_{\text {all paths, } \tau}^{n} \cap B_{\text {recogn straight }}^{n} \cap E_{\text {stop }, \tau}^{m} \subseteq E_{\text {seed II }}^{n}
$$

Proof of Lemma 6.23. Assume that the events on the left hand side of (6.30) hold. In order to show that the $E_{\text {seed II }}^{n}$ holds, let $w_{2} \in \operatorname{Seed}_{\mathrm{II}}^{n}$ (Input). We need to show that $w_{2}$ is a ladder word of $\xi\left[\left[-2 \cdot 2^{n}, 2 \cdot 2^{n}\right]\right.$. Using (4.5), we take $w_{1}, w_{3} \in \mathcal{C}^{c_{1} n}$ with $\left(w_{1}, w_{2}, w_{3}\right) \in \operatorname{Seed}_{\mathrm{I}}^{n}$ (Input); thus $w_{1} w_{2} w_{3} \equiv \eta\left\lceil\left(\tau(k)+j+\left[0,3 c_{1} n[)\right.\right.\right.$ for some $k<2^{\alpha n}$ and $j \in\left[0,7 c_{1} n l\right]$. Since $E_{\mathrm{stop}, \tau}^{m}$ holds, we have $|S(\tau(k))| \leq 2^{n}$. Using $2^{n}+7 c_{1} n l^{2}+3 c_{1} l n \leq 2 \cdot 2^{n}-c_{1} n l$, we see that the random walk $S$ is located inside the interval $\left[-2 \cdot 2^{n}+c_{1} n l, 2 \cdot 2^{n}-c_{1} n l\right]$ during the time interval $\tau(k)+7 c_{1} n l+\left[0,3 c_{1} n[\right.$. The word $w_{1} w_{2} w_{3}$ is read along an admissible piece of path, say $R \in \operatorname{AdPath}\left(2 \cdot 2^{n}-c_{1} n l, 3 c_{1} n\right)$ with $\xi \circ R=w_{1} w_{2} w_{3}$; (the time interval is shifted back to the origin). The event $E_{\text {only ladder }}^{n}$ holds by Lemma 6.19, and we have $\left(w_{1}, w_{2}, w_{3}\right) \in$ Puzzle $\mathrm{I}_{\mathrm{I}}^{n}$ (Input); hence $w_{2}$ is a ladder word of $\xi\left\lceil\left[-11 \cdot 2^{n}, 11 \cdot 2^{n}\right]\right.$; say $w_{2}=\xi \circ \pi$ for a ladder path $\pi:\left[0, c_{1} n\left[\rightarrow\left[-11 \cdot 2^{n}, 11 \cdot 2^{n}\right]\right.\right.$. Let $\pi^{\prime}=R\left\lceil\left[c_{1} n, 2 c_{1} n\right.\right.$ [ be the middle piece of $R$, along which one observes $\left(\xi \circ \pi^{\prime}\right)_{\rightarrow}=w_{2}=\xi \circ \pi$.

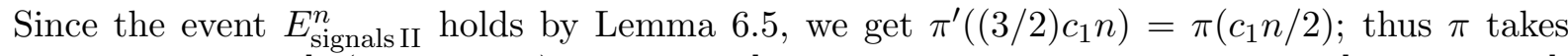
least one value in $\left[-\left(2 \cdot 2^{n}-c_{1} n l\right), 2 \cdot 2^{n}-c_{1} n l\right]$; therefore all the values of $\pi$ are in $\left[-2 \cdot 2^{n}, 2 \cdot 2^{n}\right]$. Thus $w_{2}$ is a ladder word of $\xi\left\lceil\left[-2 \cdot 2^{n}, 2 \cdot 2^{n}\right]\right.$.

For the rest of the proof we assume $l_{\rightarrow} \neq l_{\leftarrow}$ and let $u \in \operatorname{Seed}_{\mathrm{III}}^{n}$ (Input). It remains to show: $u$ is a right ladder word of $\xi\left\lceil\left[-2 \cdot 2^{n}, 2 \cdot 2^{n}\right]\right.$. Using Definition (4.6) of Seed III , we choose $v \in \operatorname{Seed}_{\mathrm{II}}^{n}$ (Input) with $\left(u\left\lceil\left(l_{\leftarrow} \mathbb{Z} \cap\left[0, c_{2} n l_{\leftarrow}\right]\right)\right)_{\rightarrow}=\left(v\left\lceil\left(l_{\rightarrow} \mathbb{Z} \cap\left[0, c_{2} n l_{\rightarrow}\right]\right)\right)_{\leftarrow}\right.\right.$. From the first part of the proof we get: $u$ and $v$ are ladder words of $\xi\left[\left[-2 \cdot 2^{n}, 2 \cdot 2^{n}\right]\right.$, since $u, v \in \operatorname{Seed}_{\mathrm{II}}^{n}$ (Input). We distinguish three cases:

1. $u$ is a right ladder word; 
2. $u$ and $v$ are left ladder words;

3. $u$ is a left ladder word and $v$ is a right ladder word.

We need to show that case 1 . holds; thus we prove that the cases 2 . and 3 . lead to a contradiction:

In case 2., let $u=\left(\xi\lceil I)_{\leftarrow}\right.$ and $v=\left(\xi\lceil J)_{\leftarrow}\right.$ for some left ladder intervals $I, J \subseteq\left[-2 \cdot 2^{n}, 2 \cdot 2^{n}\right]$, $|I|=|J|=c_{1} n$. We get $\left(u\left\lceil\left(l_{\leftarrow} \mathbb{Z} \cap\left[0, c_{2} n l_{\leftarrow}\right]\right)\right)_{\rightarrow}=\left(\xi\left\lceil I^{\prime}\right)_{\leftarrow}\right.\right.$ for some $l_{\leftarrow}^{2}$-spaced interval $I^{\prime} \subseteq I$, $\left|I^{\prime}\right|=c_{2} n+1$. Similarly, $\left(v\left\lceil\left(l_{\rightarrow} \mathbb{Z} \cap\left[0, c_{2} n l_{\rightarrow}\right]\right)\right)_{\leftarrow}=\left(\xi\left\lceil J^{\prime}\right) \rightarrow\right.\right.$ for some $l_{\leftarrow} l_{\rightarrow}$-spaced interval $J^{\prime} \subseteq J,\left|J^{\prime}\right|=c_{2} n+1$. Thus $\left(\xi\left\lceil I^{\prime}\right)_{\leftarrow}=\left(\xi\left\lceil J^{\prime}\right)_{\rightarrow}\right.\right.$, which is incompatible with the event $B_{\text {unique fit }}^{n}$.

In case 3., let $u=\left(\xi\lceil I)_{\leftarrow}\right.$ for some left ladder interval $I \subseteq\left[-2 \cdot 2^{n}, 2 \cdot 2^{n}\right]$ and $v=\left(\xi\lceil J)_{\rightarrow}\right.$ for some right ladder interval $J \subseteq\left[-2 \cdot 2^{n}, 2 \cdot 2^{n}\right],|I|=|J|=c_{1} n$. We get again $\left(u\left\lceil\left(l_{\leftarrow} \mathbb{Z} \cap\right.\right.\right.$

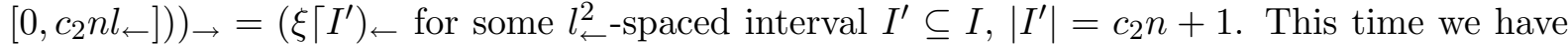
$\left(v\left\lceil\left(l_{\rightarrow} \mathbb{Z} \cap\left[0, c_{2} n l_{\rightarrow}\right]\right)\right)_{\leftarrow}=\left(\xi\left\lceil J^{\prime}\right)_{\leftarrow}\right.\right.$ for some $l_{\rightarrow}^{2}$-spaced interval $J^{\prime} \subseteq J,\left|J^{\prime}\right|=c_{2} n+1$. Since $l_{\leftarrow}^{2} \neq l_{\rightarrow}^{2}$, we have $I^{\prime} \neq J^{\prime}$. We obtain $\left(\xi\left\lceil I^{\prime}\right)_{\leftarrow}=\left(\xi\left\lceil J^{\prime}\right)_{\leftarrow}\right.\right.$, which is incompatible with the event $B_{\text {unique fit }}^{n}$.

Thus cases 2. and 3. cannot occur. Summarizing, we have proven that the event $E_{\text {seed II }}^{n}$ holds.

Definition 6.24 If $l_{\rightarrow}=l_{\leftarrow}$, we define the event

$E_{\text {dist }}^{n}:=$

$\left\{\begin{array}{l}\text { For all ladder intervals } I, J \subseteq\left[-9 \cdot 2^{n}, 9 \cdot 2^{n}\right],|I|=|J|=c_{1} n \text { : if at least one } \\ \text { of }\left(\left(\xi\lceil I)_{\rightarrow},\left(\xi\lceil J)_{\rightarrow}\right),\left(\left(\xi\lceil I)_{\rightarrow},\left(\xi\lceil J)_{\leftarrow}\right),\left(\left(\xi\lceil I)_{\leftarrow},\left(\xi\lceil J)_{\rightarrow}\right), \text { or }\left(\left(\xi\lceil I)_{\leftarrow},\left(\xi\lceil J)_{\leftarrow}\right) \text { is in }\right.\right.\right.\right.\right.\right.\right.\right. \\ \text { Neighbors }{ }^{n}(\text { Input }), \text { then distance }(I, J) \leq 3 \cdot l c_{1} n .\end{array}\right\}$

In the case $l_{\rightarrow} \neq l_{\leftarrow}$, we set $E_{\text {dist }}^{n}$ to be the sure event.

Lemma 6.25 $B_{\text {signals }}^{n} \cap E_{\text {stop }, \tau}^{m} \subseteq E_{\text {dist }}^{n}$

Proof of Lemma 6.25. Assume that the event $B_{\text {signals }}^{n} \cap E_{\text {stop }, \tau}^{m}$ holds, and that $l_{\rightarrow}=l_{\leftarrow}=l$. Let $I, J \subseteq\left[-9 \cdot 2^{n}, 9 \cdot 2^{n}\right],|I|=|J|=c_{1} n$, be right ladder intervals, and assume that there is a $\left(w_{1}, w_{2}\right)$ among $\left(\left(\xi\lceil I)_{\rightarrow},\left(\xi\lceil J)_{\rightarrow}\right),\left(\left(\xi\lceil I)_{\rightarrow},\left(\xi\lceil J)_{\leftarrow}\right),\left(\left(\xi\lceil I)_{\leftarrow},\left(\xi\lceil J)_{\rightarrow}\right)\right.\right.\right.\right.\right.\right.$, or $\left(\left(\xi\lceil I)_{\leftarrow},\left(\xi\lceil J)_{\leftarrow}\right)\right.\right.$ with $\left(w_{1}, w_{2}\right) \in$ Neighbors $^{n}\left(\right.$ Input). By definition (4.7), some word $w_{1} w w_{2}$ with $w \in \mathcal{C}^{h-1}$ occurs in the observations $\chi$ at most $2^{2 n}$ time steps after a stopping time $\tau(k), k<2^{\alpha n}$. Since $E_{\text {stop }, \tau}^{m}$ holds, the random walk remains in the interval $\left[-2 \cdot l 2^{2 n}, 2 \cdot l 2^{2 n}\right]$ during that time interval; say the random walk follows an admissible piece of path $R:\left[0,2 c_{1} n+h-1\left[\rightarrow\left[-2 \cdot l 2^{2 n}, 2 \cdot l 2^{2 n}\right]\right.\right.$ while producing the observations $\xi \circ R=w_{1} w w_{2}$; (we shifted the time domain back to the origin). $R$ consists of the three pieces $\pi_{1}^{\prime}=R\left\lceil\left[0, c_{1} n\left[, \pi^{\prime}=R\left\lceil\left(c_{1} n+\left[0, h-1[)\right.\right.\right.\right.\right.\right.$, and $\pi_{2}^{\prime}=R\left\lceil\left(c_{1} n+h-1+\left[0, c_{1} n[)\right.\right.\right.$ with $\xi \circ \pi_{1}^{\prime}=w_{1},\left(\xi \circ \pi^{\prime}\right)_{\rightarrow}=w$, and $\left(\xi \circ \pi_{2}^{\prime}\right)_{\rightarrow}=w_{2}$. Let $x_{1}:=c_{1} n / 2$ and $x_{2}:=(3 / 2) c_{1} n+h-1$ be the points in the middle of the domain of $\pi_{1}^{\prime}$ and $\pi_{2}^{\prime}$, respectively. Then

$$
\left|\pi_{1}^{\prime}\left(x_{1}\right)-\pi_{2}^{\prime}\left(x_{2}\right)\right| \leq\left(c_{1} n+h-1\right) l,
$$

since the path $R$ cannot travel faster than $l$ per step. The event $E_{\text {signals II }}^{n}$ holds by Lemma 6.5. Let $\pi_{1}:\left[0, c_{1} n\left[\rightarrow I\right.\right.$ and $\pi_{2}: c_{1} n+h-1+\left[0, c_{1} n[\rightarrow J\right.$ be ladder paths with range $I$ and $J$, respectively; we choose these paths to be left or right ladder paths according to whether the reading direction is " $\longleftarrow$ " or " $\rightarrow$ ". Hence, using $\xi \circ \pi_{1}=w_{1}=\xi \circ \pi_{1}^{\prime}$ and $\left(\xi \circ \pi_{2}\right)_{\rightarrow}=w_{2}=\left(\xi \circ \pi_{2}^{\prime}\right)_{\rightarrow}$, we obtain $\pi_{1}^{\prime}\left(x_{1}\right)=\pi_{1}\left(x_{1}\right)$ and $\pi_{2}^{\prime}\left(x_{2}\right)=\pi_{2}\left(x_{2}\right)$. Consequently (6.32) implies

$$
\operatorname{distance}(I, J) \leq\left|\pi_{1}\left(x_{1}\right)-\pi_{2}\left(x_{2}\right)\right| \leq 3 \cdot l c_{1} n \text {. }
$$


Summarizing, we have shown that the event $E_{\text {dist }}^{n}$ holds.

The following event $E_{\text {mod } \gamma \text { ok }}^{n}$ compares modulo classes (modulo some $\gamma$ ) in "reconstructed" pieces $w$ with modulo classes in the "true" scenery $\xi$. Roughly speaking, it states that all modulo classes are reconstructed correctly, and either all of them are reconstructed in the correct orientation ("case A"), or all of them are reversed ("case B"). Even more, reversion is only allowed for symmetric maximal jumps of the random walk. Our goal is to show that this event holds for $\gamma=1$ (at least if the basic events $B \ldots$ hold), but as intermediate steps, other values of $\gamma$ are relevant, too.

Definition 6.26 For all divisors $\gamma \geq 1$ of $l_{\rightarrow}$, we define the event

$E_{\bmod \gamma \text { ok }}^{n}:=$

$$
\left\{\begin{array}{l}
\text { For all } w \in \text { SolutionPieces }{ }^{n} \text { (Input) there is a bijection } \iota_{\gamma}: \mathbb{Z} / \gamma \mathbb{Z} \rightarrow \mathbb{Z} / \gamma \mathbb{Z} \text { such } \\
\text { that (at least) one of the following two cases holds: } \\
\text { A) } \forall Z \in \mathbb{Z} / \gamma \mathbb{Z}: \quad \xi\left\lceil( [ - 2 ^ { n } , 2 ^ { n } ] \cap \iota _ { \gamma } ( Z ) ) \sqsubseteq w \left\lceilZ \sqsubseteq \xi \left\lceil\left(\left[-9 \cdot 2^{n}, 9 \cdot 2^{n}\right] \cap \iota_{\gamma}(Z)\right)\right.\right.\right. \\
\text { B) } l_{\rightarrow}=l_{\leftarrow} \text { and } \\
\quad \forall Z \in \mathbb{Z} / \gamma \mathbb{Z}: \quad \xi\left\lceil( [ - 2 ^ { n } , 2 ^ { n } ] \cap \iota _ { \gamma } ( Z ) ) \sqsubseteq \left(w \lceil Z ) \leftrightarrow \sqsubseteq \xi \left\lceil\left(\left[-9 \cdot 2^{n}, 9 \cdot 2^{n}\right] \cap \iota_{\gamma}(Z)\right)\right.\right.\right.
\end{array}\right\} .
$$

Lemma 6.27 For $\gamma=l_{\rightarrow}$, we have $E_{\text {seed II }}^{n} \cap E_{\text {mod class }}^{n} \cap E_{\text {dist }}^{n} \cap B_{\text {unique fit }}^{n} \subseteq E_{\text {mod } l_{\rightarrow \text { ok }}}^{n}$.

Proof of Lemma 6.27. Assume that the event $E_{\text {seed II }}^{n} \cap E_{\text {mod class }}^{n} \cap E_{\text {dist }}^{n} \cap B_{\text {unique fit }}^{n}$ holds. Let $w \in$ SolutionPieces ${ }^{n}$ (Input). Let $Z \in \mathbb{Z} / l_{\rightarrow} \mathbb{Z}$. In order to define $\iota(Z)=\iota_{l_{\rightarrow}}(Z)$, we proceed as follows: Since $w \in$ Filter $_{4}$ (Input), there exists a right ladder interval $I \subseteq Z \cap\left[-2 \cdot 2^{n}, 2 \cdot 2^{n}\right]$ such that $\left(w\lceil I)_{\rightarrow} \in \operatorname{Seed}_{\mathrm{III}}^{n}(\tau, \eta)\right.$. We choose such an $I$. Then $\left(w\lceil I)_{\rightarrow}\right.$ is a left or right ladder word of $\xi\left[\left[-2 \cdot 2^{n}, 2 \cdot 2^{n}\right]\right.$, since the event $E_{\text {seed II }}^{n}$ holds. More specifically: for some right ladder interval $J \subseteq\left[-2 \cdot 2^{n}, 2 \cdot 2^{n}\right]$, at least one of the following two cases holds true:

Case $\mathrm{A}(Z): \quad w\lceil I \equiv \xi\lceil J$,

Case $\mathrm{B}(Z): \quad l_{\rightarrow}=l_{\leftarrow}$ and $(w\lceil I) \leftrightarrow \equiv \xi\lceil J$.

We define $\iota(Z):=J+l_{\rightarrow} \mathbb{Z} \in \mathbb{Z} / l_{\rightarrow} \mathbb{Z}$. Since the event $E_{\text {mod class }}^{n}$ holds, we get

for Case $\mathrm{A}(Z): \quad \xi\left\lceil\left(\left[-2^{n}, 2^{n}\right] \cap \iota(Z)\right) \sqsubseteq w\left\lceil Z \sqsubseteq \xi\left\lceil\left(\left[-9 \cdot 2^{n}, 9 \cdot 2^{n}\right] \cap \iota(Z)\right)\right.\right.\right.$, for Case $\mathrm{B}(Z): \quad \xi\left\lceil\left(\left[-2^{n}, 2^{n}\right] \cap \iota(Z)\right) \sqsubseteq\left(w\lceil Z) \leftrightarrows \xi\left\lceil\left(\left[-9 \cdot 2^{n}, 9 \cdot 2^{n}\right] \cap \iota(Z)\right)\right.\right.\right.$.

We claim that one of the following two cases occurs:

Case A: $\quad$ For all modulo classes $Z \in \mathbb{Z} / l_{\rightarrow} \mathbb{Z}$ holds Case $\mathrm{A}(Z)$;

Case B: For all modulo classes $Z \in \mathbb{Z} / l_{\rightarrow} \mathbb{Z}$ holds Case $\mathrm{B}(Z)$.

This is obvious for $l_{\rightarrow} \neq l_{\leftarrow}$, since then Case $\mathrm{B}(Z)$ cannot occur. To prove the claim for $l_{\rightarrow}=l_{\leftarrow}$, we proceed as follows: For $Z \in \mathbb{Z} / \mathbb{Z}$, let $T_{Z}: \mathbb{Z} \rightarrow \mathbb{Z}$ denote a translation (Case $\mathrm{A}(Z)$ ) or reflection (Case $\mathrm{B}(Z))$ which transports $w\left\lceil Z\right.$ to a subpiece of $\xi\left\lceil\left[-9 \cdot 2^{n}, 9 \cdot 2^{n}\right] \cap \iota(Z)\right.$. Let $Z, W \in$ $\mathbb{Z} / l \mathbb{Z}$. We choose two right ladder intervals $I_{1} \subseteq Z \cap\left[4 \cdot 2^{n}, 5 \cdot 2^{n}\right], I_{2} \subseteq W \cap\left[4 \cdot 2^{n}, 5 \cdot 2^{n}\right],\left|I_{1}\right|=$ $\left|I_{2}\right|=c_{1} n$, with $I_{1} \triangleright_{n} I_{2}$; such intervals exist, since $\operatorname{supp} \mu^{* h}$ meets every modulo class (modulo $l$ ) and since $n \geq n_{0}$ is large enough. We abbreviate $I_{1}^{\prime}:=T_{Z}\left[I_{1}\right]$ and $I_{2}^{\prime}:=T_{W}\left[I_{2}\right]$. Since $w \in$ Filter $_{2}$ (Input) one has $\left(\left(w\left\lceil I_{1}\right)_{\rightarrow},\left(w\left\lceil I_{2}\right)_{\rightarrow}\right) \in\right.\right.$ Neighbors $^{n}$ (Input). Let $X_{Z}$ denote the symbol " $\rightarrow$ " in the Case $\mathrm{A}(Z)$ and " $\leftarrow$ " in the Case $\mathrm{B}(Z)$. Then $\left(\left(w\left\lceil I_{1}\right)_{\rightarrow},\left(w\left\lceil I_{2}\right)_{\rightarrow}\right)=\left(\left(\xi\left\lceil I_{1}^{\prime}\right)_{X_{Z}},\left(\xi\left\lceil I_{2}^{\prime}\right)_{X_{W}}\right)\right.\right.\right.\right.$. Since the event $E_{\text {dist }}^{n}$ holds, this implies distance $\left(I_{1}^{\prime}, I_{2}^{\prime}\right) \leq 3 \cdot l c_{1} n$. However, $T_{Z}$ maps $\left[-5 \cdot 2^{n}, 5 \cdot 2^{n}\right]$ 
to $\left[-9 \cdot 2^{n}-l, 9 \cdot 2^{n}+l\right]$; (the extra summand $l$ arises since $T_{Z}$ was specified only by its action on a modulo class). Thus it maps $I_{1}, I_{2} \subseteq\left[4 \cdot 2^{n}, 5 \cdot 2^{n}\right]$ to a subset of $\left[4 \cdot 2^{n}-l, 9 \cdot 2^{n}+l\right]$ in the Case $\mathrm{A}(Z)$, and to a subset of $\left[-9 \cdot 2^{n}-l,-4 \cdot 2^{n}+l\right]$ in the Case $\mathrm{B}(Z)$. The same statement holds with $Z$ replaced by $W$. The intervals $\left[4 \cdot 2^{n}-l, 9 \cdot 2^{n}+l\right]$ and $\left[-9 \cdot 2^{n}-l,-4 \cdot 2^{n}+l\right]$ are farther apart than $3 \cdot l c_{1} n \geq$ distance $\left(I_{1}^{\prime}, I_{2}^{\prime}\right)$; thus either both $T_{Z}$ and $T_{W}$ must be translations, or both must be reflections. Summarizing, we have shown so far that Case A holds or Case B holds.

It only remains to show that $\iota: \mathbb{Z} / l_{\rightarrow} \mathbb{Z} \rightarrow \mathbb{Z} / l_{\rightarrow} \mathbb{Z}$ is bijective. Since $\mathbb{Z} / l_{\rightarrow} \mathbb{Z}$ is finite, it suffices to show that $\iota$ is injective: Let $Z, W \in \mathbb{Z} / l_{\rightarrow} \mathbb{Z}$ with $\iota(Z)=\iota(W)$. Using the above maps $T_{Z}$, $T_{W}$ again, we know

$$
\begin{array}{rcc}
T_{Z}[Z \cap \operatorname{domain}(w)] & =T_{Z}\left[Z \cap\left[-5 \cdot 2^{n}, 5 \cdot 2^{n}\right]\right] \subseteq & \iota(Z) \cap\left[-9 \cdot 2^{n}, 9 \cdot 2^{n}\right], \\
T_{W}[W \cap \operatorname{domain}(w)] & =T_{W}\left[W \cap\left[-5 \cdot 2^{n}, 5 \cdot 2^{n}\right]\right] \subseteq & \iota(W) \cap\left[-9 \cdot 2^{n}, 9 \cdot 2^{n}\right] .
\end{array}
$$

The sets on the right hand of (6.35) and (6.36) coincide; thus $T_{Z}\left[Z \cap\left[-5 \cdot 2^{n}, 5 \cdot 2^{n}\right]\right]$ and $T_{W}\left[W \cap\left[-5 \cdot 2^{n}, 5 \cdot 2^{n}\right]\right]$ overlap at least in $K \cap \iota(Z)$ for some interval $K$ of length $2^{n}$. We choose any right ladder interval $D \subseteq K \cap \iota(Z)$ with $|D|=c_{1} n$ and set $D_{1}:=T_{Z}^{-1}[D]$ and $D_{2}:=T_{W}^{-1}[D]$. Then

$$
\begin{array}{ll}
\text { Case A: } & \left(w\left\lceil D_{1}\right)_{\rightarrow}=\left(\xi\lceil D)_{\rightarrow}=\left(w\left\lceil D_{2}\right)_{\rightarrow},\right.\right.\right. \\
\text { Case B: } & \left(w\left\lceil D_{1}\right)_{\rightarrow}=\left(\xi\lceil D)_{\leftarrow}=\left(w\left\lceil D_{2}\right)_{\rightarrow} ;\right.\right.\right.
\end{array}
$$

thus $w \in$ Filter $_{5}$ (Input) implies $D_{1}=D_{2}$; hence $Z=D_{1}+l_{\rightarrow} \mathbb{Z}=D_{2}+l_{\rightarrow} \mathbb{Z}=W$. This shows that $\iota$ is indeed injective.

The next lemma contains a "step down" procedure in order to arrange correctly larger and larger modulo classes in a reconstructed piece of scenery $w$. Here is a rough idea for the rather complex construction:

Suppose we have already correctly reconstructed large pieces of the scenery $\xi$ restricted to modulo classes $(\bmod \gamma$, say) up to a translation (and possibly a global reflection for all classes). Our task is to identify the relative translation between different modulo classes.

We start with a "reference" ladder word; it occurs over both, a ladder interval $I$ in the reconstructed "candidate" scenery $w$, and a ladder interval $J$ in the "true" scenery $\xi$ (possibly reflected). Then we look for the rightmost "neighboring" ladder words that occur not in the same modulo class as the reference word, both in the candidate scenery and in the true scenery; we use here the "estimated" neighborship relation "Neighbors". Taking the rightmost "neighboring" words as our new starting point, we repeat this construction until we are sure after $\gamma$ steps to re-enter the modulo class that we started with; say we arrive at ladder intervals $I_{\gamma}$ and $J_{\gamma}$, respectively. In this way we obtain two "chains" $\left(I_{i}\right)$ and $\left(J_{i}\right)$ of neighboring ladder intervals; $\left(J_{i}\right)$ belongs to the the "true" scenery, and $\left(I_{i}\right)$ belongs to the "reconstructed candidate" $w$.

Using the Definition of the tests "Filter $2 / 3$ ", and of the events $E_{\text {neighbor I/II }}$, we know that the "estimated" and the "geometric" neighborship relations coincide at least when taking only rightmost neighbors as above; this holds for both, the "reconstructed" piece $w$ and for the "true" scenery $\xi$. The distance between $I_{\gamma}$ and $I$ equals the distance between $J_{\gamma}$ and $J$, since this distance is not affected by a relative translation between different modulo classes; recall that $I_{\gamma}$ and $I$ belong to the same class modulo $\gamma$, and so do $J_{\gamma}$ and $J$. Having identified the starting point and the end point of our two chains of intervals, there also no ambiguity left for the relative position of the intervals in between in the chain; but then we have successfully reconstructed the larger modulo class spanned by the whole chain $\left(I_{i}\right)$. 
This construction is repeated recursively until we have correctly reconstructed the whole piece of scenery.

We describe the procedure formally:

Lemma 6.28 Assume that the events $B_{\text {unique fit, }}^{n} E_{\text {neighbor I }}^{n}$, and $E_{\text {neighbor II }}^{n}$ hold true. Let $\gamma>1$ be a divisor of $l_{\rightarrow}$ and assume that the event $E_{\bmod \gamma \text { ok }}^{n}$ is valid. Then there is a divisor $\gamma^{\prime}$ of $l_{\rightarrow}$ with $1 \leq \gamma^{\prime}<\gamma$ such that the event $E_{\bmod \gamma^{\prime} \text { ok }}^{n}$ is valid, too.

Proof. Let $\gamma$ be as in the hypothesis of the lemma. Every modulo class $Z \in \mathbb{Z} / \gamma \mathbb{Z}$ is a union of modulo classes $Z^{\prime} \in \mathbb{Z} / l_{\rightarrow} \mathbb{Z}$. Furthermore, every such modulo class $Z^{\prime} \in \mathbb{Z} / l_{\rightarrow} \mathbb{Z}$ has a nonempty intersection with supp $\mu^{* h}$. (One can see this as follows: Since 1 is the greatest common divisor of the elements of $\operatorname{supp} \mu$, every integer can be written in the form $\beta l_{\rightarrow}+\sum_{j=1}^{K} s_{j}$ with $\beta \in \mathbb{Z}$, $K \in \mathbb{N}$, and $s_{j} \in \operatorname{supp} \mu$ for $1 \leq j \leq K$. By Lemma 6.11 it suffices to take $K=h$; thus we get $\mathbb{Z}=\operatorname{supp} \mu^{* h}+l_{\rightarrow} \mathbb{Z}$, which is equivalent to the above claim.)

Since we assume $\gamma>1$, the set difference $\mathbb{Z} \backslash \gamma \mathbb{Z}$ contains at least one $Z \in \mathbb{Z} / \gamma \mathbb{Z}$ as a subset; thus $\mathbb{Z} \backslash \gamma \mathbb{Z}$ has at least one element in common with supp $\mu^{* h}$. Let $M_{\rightarrow}:=\max [(\mathbb{Z} \backslash \gamma \mathbb{Z}) \cap$ $\left.\operatorname{supp} \mu^{* h}\right]$ and $M_{\leftarrow}:=-\min \left[(\mathbb{Z} \backslash \gamma \mathbb{Z}) \cap \operatorname{supp} \mu^{* h}\right]$. Define $\gamma^{\prime}$ to be the greatest common divisor of $\gamma$ and $M_{\rightarrow}$; thus $\gamma^{\prime}<\gamma$ since $M_{\rightarrow} \notin \gamma \mathbb{Z}$.

Let $w \in$ SolutionPieces $^{n}$ (Input). According to Definition (6.34) of $E_{\bmod \gamma \text { ok }}^{n}$ we have to distinguish two cases A and B; however, we treat both cases simultaneously as far as possible. We set

$$
\zeta:= \begin{cases}\xi\left[\left[-9 \cdot 2^{n}, 9 \cdot 2^{n}\right]\right. & \text { in case A of }(6.34), \\ \left(\xi\left[\left[-9 \cdot 2^{n}, 9 \cdot 2^{n}\right]\right) \leftrightarrow\right. & \text { in case B of }(6.34) .\end{cases}
$$

For $Z \in \mathbb{Z} / \gamma Z$ we set $\tilde{\iota}_{\gamma}(Z):= \pm \iota_{\gamma}( \pm Z)$ with "+" in case A and "-" in case B; here the bijection $\iota_{\gamma}: \mathbb{Z} / \gamma \mathbb{Z} \rightarrow \mathbb{Z} / \gamma \mathbb{Z}$ is taken from Definition (6.34) of the event $E_{\bmod \gamma \text { ok }}^{n}$. The introduction of $\tilde{\iota}_{\gamma}$ takes care of the inversion of modulo classes in $\zeta$ in case B. Since the event $E_{\bmod \gamma \text { ok }}^{n}$ is valid, we have for all $Z \in \mathbb{Z} / \gamma \mathbb{Z}$ :

$$
\zeta\left\lceil( \tilde { \iota } _ { \gamma } ( Z ) \cap [ - 2 ^ { n } , 2 ^ { n } ] ) \sqsubseteq w \left\lceilZ \sqsubseteq \zeta \left\lceil\tilde{\iota}_{\gamma}(Z) .\right.\right.\right.
$$

For $Z \in \mathbb{Z} / \gamma \mathbb{Z}$, let $T_{Z}: \mathbb{Z} \rightarrow \mathbb{Z}$ denote the translation which transports $w\left\lceil Z\right.$ to some $T_{Z}[w\lceil Z] \subseteq$ $\zeta\left[\tilde{\iota}_{\gamma}(Z)\right.$; in particular $T_{Z}[Z]=\tilde{\iota}_{\gamma}(Z)$. $T_{Z}$ is uniquely determined, since the event $B_{\text {unique fit }}^{n}$ holds. Of course, $T_{Z}$ also depends on $\gamma$, but we suppress this in the notation, since $\gamma$ is considered fixed for the moment. For $W \in \mathbb{Z} / \gamma \mathbb{Z}$, we set $\tilde{T}_{W}:=\left(T_{\tilde{\iota}_{\gamma}^{-1}(W)}\right)^{-1}$; thus $\tilde{T}_{W}[W]=\tilde{\iota}_{\gamma}^{-1}(W)$. For later use, we note

$$
\zeta\left\lceil( \tilde { \iota } _ { \gamma } ( Z ) \cap [ - 2 ^ { n } , 2 ^ { n } ] ) \subseteq T _ { Z } \left[w \lceil Z ] \subseteq \zeta \left\lceil\tilde{\iota}_{\gamma}(Z) .\right.\right.\right.
$$

We define

$$
\zeta^{\prime}:=\bigcup_{Z \in \mathbb{Z} / \gamma \mathbb{Z}} T_{Z}[w\lceil Z] \subseteq \zeta .
$$

Note that $\left[-2^{n}, 2^{n}\right] \subseteq \operatorname{domain}\left(\zeta^{\prime}\right)$. For (nonempty) ladder intervals $I$ and $J$, we abbreviate $T_{I}:=T_{I+\gamma \mathbb{Z}}$ and $\tilde{T}_{J}:=\tilde{T}_{J+\gamma \mathbb{Z}}$.

Let the following data be given: $u \in\left\{w, \zeta^{\prime}\right\}$, a right ladder interval $I$ contained in the domain of $u$ with $|I|=c_{1} n$, and $k \in[0, \gamma]$. We define $\operatorname{Seq}(I, u, k)$ to denote the set of all $\left(I_{0}, \ldots, I_{k}\right)$ with the following properties:

1. $I_{0}=I$

2. $I_{0}, \ldots, I_{k}$ are right ladder intervals contained in the domain of $u$ with $\left|I_{j}\right|=c_{1} n, 0 \leq j \leq k$. 
3. For all $j \in\left[0, k\left[: I_{j}+\gamma \mathbb{Z} \neq I_{j+1}+\gamma \mathbb{Z}\right.\right.$.

4. For all $j \in\left[0, k\left[:\left(\left(u\left\lceil I_{j}\right)_{\rightarrow},\left(u\left\lceil I_{j+1}\right)_{\rightarrow}\right) \in\right.\right.\right.\right.$ Neighbors $^{n}$ (Input).

Of course $\operatorname{Seq}(I, u, k)$ also depends on $\gamma$, Input, and $n$, but these parameters are considered fixed for the moment.

Let $\operatorname{Max} \operatorname{Seq}(I, u, k)$ denote the set of all $\left(I_{j}\right)_{j=0, \ldots, k} \in \operatorname{Seq}(I, u, k)$ for which $\min I_{k}-\min I_{0}$ is maximal.

Given a modulo class $Z \in \mathbb{Z} / \gamma \mathbb{Z}$, we take a fixed right ladder interval $J \subseteq \tilde{\iota}_{\gamma}^{-1}(Z) \cap\left[0,\left(c_{1} n+\right.\right.$ $1) l_{\rightarrow} \subseteq \tilde{\iota}_{\gamma}^{-1}(Z) \cap \operatorname{domain}\left(\zeta^{\prime}\right),|J|=c_{1} n$. Furthermore, we set $I:=\tilde{T}_{J} J \subseteq Z \cap \operatorname{domain}(w)$.

$J$ serves as a "reference" interval in the "true" (only possibly reflected) piece of scenery $\zeta$ ', while $I$ serves as a "reference" interval in the "reconstructed" piece of scenery $w$.

We prove by induction over $k$ :

- $\operatorname{MaxSeq}(I, w, k)$ contains a unique element $\left(I_{j}\right)_{j=0, \ldots, k}$, namely

$$
I_{j}=M_{\rightarrow} j+\left(c_{1} n-1\right) l_{\rightarrow} j+I .
$$

- $\operatorname{MaxSeq}\left(J, \zeta^{\prime}, k\right)$ contains a unique element $\left(J_{j}\right)_{j=0, \ldots, k}$, too, namely

$$
J_{j}=M j+\left(c_{1} n-1\right) l_{\rightarrow} j+J,
$$

where $M=M_{\rightarrow}$ in case $\mathrm{A}$ and $M=M_{\leftarrow}$ in case $\mathrm{B}$.

This is obvious for $k=0$. Here is the induction step $k-1 \mapsto k$ :

If $\left(I_{j}\right)_{j=0, \ldots, k},\left(J_{j}\right)_{j=0, \ldots, k}$ are given by (6.41) and (6.42), then

$$
\left(I_{j}\right)_{j=0, \ldots, k} \in \operatorname{Seq}(I, w, k) \quad \text { and } \quad\left(J_{j}\right)_{j=0, \ldots, k} \in \operatorname{Seq}\left(J, \zeta^{\prime}, k\right) .
$$

To see this, we check the conditions 1.-4. in the definition of Seq:

1. This is obvious.

2. The only nontrivial claims are $J_{j} \subseteq \operatorname{domain}\left(\zeta^{\prime}\right)$ and $I_{j} \subseteq$ domain $(w), 0 \leq j \leq k$. To prove the first claim, we observe $\left|\min J-\min J_{j}\right| \leq\left(M+c_{1} n l\right) k \leq 2 c_{1} n l \gamma \leq 2 c_{1} n l^{2}$; thus we obtain for all $i \in J_{j}:|i| \leq 2 c_{1} n l^{2}+\left(c_{1} n+1\right) l_{\rightarrow} \leq 2^{n}$; hence $J_{j} \subseteq\left[-2^{n}, 2^{n}\right] \subseteq \operatorname{domain}\left(\zeta^{\prime}\right)$. To prove the second claim, we observe that $J+\left[-2^{n} / 2,2^{n} / 2\right] \subseteq\left[-2^{n}, 2^{n}\right]=\operatorname{domain}\left(\zeta^{\prime}\right)$ (recall $n \geq n_{0}$, and $n_{0}$ is large enough). We apply the translation $\tilde{T}_{J}$ to $J+\left(\left[-2^{n} / 2,2^{n} / 2\right] \cap \gamma \mathbb{Z}\right)$ to obtain $I+\left(\left[-2^{n} / 2,2^{n} / 2\right] \cap \gamma \mathbb{Z}\right)=\tilde{T}_{J}\left[J+\left(\left[-2^{n} / 2,2^{n} / 2\right] \cap \gamma \mathbb{Z}\right)\right] \subseteq \operatorname{domain}(w)=\left[-5 \cdot 2^{n}, 5 \cdot 2^{n}\right]$. This implies $I+\left[-2^{n} / 2+\gamma, 2^{n} / 2-\gamma\right] \subseteq\left[-5 \cdot 2^{n}, 5 \cdot 2^{n}\right]$, since $\left[-5 \cdot 2^{n}, 5 \cdot 2^{n}\right]$ is an interval; consequently $I_{j} \subseteq I+\left[-2^{n} / 2+\gamma, 2^{n} / 2-\gamma\right] \subseteq$ domain $(w)$, which proves the second claim.

3. This is a consequence of $\min I_{j+1}-\max I_{j}=M \rightarrow \notin \gamma \mathbb{Z}$ and $\min J_{j+1}-\max J_{j}=M \notin \gamma \mathbb{Z}$.

4. Because of $\min I_{j+1}-\max I_{j}=M_{\rightarrow} \in \operatorname{supp} \mu^{* h}$ we get $I_{j} \triangleright_{n} I_{j+1}$; thus the fact $w \in$ Filter $_{2}^{n}$ (Input) implies $\left(\left(w\left\lceil I_{j}\right)_{\rightarrow},\left(w\left\lceil I_{j+1}\right)_{\rightarrow}\right) \in\right.\right.$ Neighbors $^{n}$ (Input); see Definition 4.4. Similarly $\min J_{j+1}-\max J_{j}=M_{\rightarrow} \in \operatorname{supp} \mu^{* h}$ in case A and $\min J_{j+1}-\max J_{j}=M_{\leftarrow} \epsilon$ $-\operatorname{supp} \mu^{* h}$ in case B. Hence we get $J_{j} \triangleright_{n} J_{j+1}$ in case A and $-J_{j} \triangleleft_{n}-J_{j+1}$ in case B; this implies $\left(\left(\zeta^{\prime}\left\lceil J_{j}\right)_{\rightarrow},\left(\zeta^{\prime}\left\lceil J_{j+1}\right)_{\rightarrow}\right) \in\right.\right.$ Neighbors $^{n}$ (Input) in both cases, since the event $E_{\text {neighbor I }}^{n}$ holds; see Definition 6.9. 
Thus the conditions 1.-4. are indeed valid.

To check the defining property of MaxSeq, consider another sequence

$$
\left(I_{j}^{\prime}\right)_{j=0, \ldots, k} \in \operatorname{Seq}(I, w, k) \quad \text { and } \quad\left(J_{j}^{\prime}\right)_{j=0, \ldots, k} \in \operatorname{Seq}\left(J, \zeta^{\prime}, k\right) .
$$

Using our induction hypotheses

$$
\begin{aligned}
\operatorname{Max} \operatorname{Seq}(I, w, k-1) & =\left\{\left(I_{j}\right)_{j=0, \ldots, k-1}\right\} \\
\operatorname{MaxSeq}\left(J, \zeta^{\prime}, k-1\right) & =\left\{\left(J_{j}\right)_{j=0, \ldots, k-1}\right\}
\end{aligned}
$$

and

$$
\left(I_{j}^{\prime}\right)_{j=0, \ldots, k-1} \in \operatorname{Seq}(I, w, k-1), \quad\left(J_{j}^{\prime}\right)_{j=0, \ldots, k-1} \in \operatorname{Seq}\left(J, \zeta^{\prime}, k-1\right),
$$

we know

$$
\begin{aligned}
\min I_{k-1}-\min I_{0} & \geq \min I_{k-1}^{\prime}-\min I_{0}^{\prime}, \\
\min J_{k-1}-\min J_{0} & \geq \min J_{k-1}^{\prime}-\min J_{0}^{\prime},
\end{aligned}
$$

with equality only if $\left(I_{j}^{\prime}\right)_{j=0, \ldots, k-1}=\left(I_{j}\right)_{j=0, \ldots, k-1}$ or $\left(J_{j}^{\prime}\right)_{j=0, \ldots, k-1}=\left(J_{j}\right)_{j=0, \ldots, k-1}$.

We treat first case of the I's: Using $\left(\left(w\left\lceil I_{k-1}^{\prime}\right)_{\rightarrow},\left(w\left\lceil I_{k}^{\prime}\right)_{\rightarrow}\right) \in\right.\right.$ Neighbors $^{n}$ (Input) and $w \in$ Filter $_{3}^{n}$ (Input) we get $I_{k-1}^{\prime} \triangleright_{n} I_{k}^{\prime}+a l_{\rightarrow}$ for some $a \in \mathbb{N}$; thus

$$
\min I_{k}^{\prime}-\max I_{k-1}^{\prime} \leq \min I_{k}^{\prime}+a l_{\rightarrow}-\max I_{k-1}^{\prime} \leq M_{\rightarrow}
$$

by the maximality of $M_{\rightarrow}$ and $I_{k}+\gamma \mathbb{Z} \neq I_{k-1}+\gamma \mathbb{Z}$; (see condition 3 . in the definition of Seq, and recall $l_{\rightarrow} \in \gamma \mathbb{Z}$ ). Hence

$$
\begin{aligned}
\min I_{k}^{\prime}-\min I_{0}^{\prime} & =\left(\min I_{k}^{\prime}-\max I_{k-1}^{\prime}\right)+\left(c_{1} n-1\right) l_{\rightarrow}+\left(\min I_{k-1}^{\prime}-\min I_{0}^{\prime}\right) \\
& \leq M_{\rightarrow}+\left(c_{1} n-1\right) l_{\rightarrow}+\left(\min I_{k-1}-\min I_{0}\right)=\min I_{k}-\min I_{0} .
\end{aligned}
$$

This proves

$$
\left(I_{j}\right)_{j=0, \ldots, k} \in \operatorname{Max} \operatorname{Seq}(I, w, k) .
$$

Furthermore, using our induction hypothesis, equality in (6.51) can hold only if $\left(I_{j}^{\prime}\right)_{j=0, \ldots, k-1} \in$ $\operatorname{MaxSeq}(I, w, k-1)$ and $\min I_{k}^{\prime}-\max I_{k-1}^{\prime}=M_{\rightarrow}$, which is equivalent to $\left(I_{j}^{\prime}\right)_{j=0, \ldots, k}=\left(I_{j}\right)_{j=0, \ldots, k}$.

We treat $\left(J_{j}\right)_{j=0, \ldots, k}$ similarly: Since the event $E_{\text {neighbor II }}^{n}$ holds, $\left(\left(\zeta^{\prime}\left\lceil J_{k-1}^{\prime}\right)_{\rightarrow},\left(\zeta^{\prime}\left\lceil J_{k}^{\prime}\right)_{\rightarrow}\right) \in\right.\right.$ Neighbors ${ }^{n}$ (Input) implies

$$
\begin{aligned}
J_{k-1}^{\prime} \triangleright_{n} J_{k}^{\prime}+a l_{\rightarrow} & \text { in case A, } \\
-J_{k-1}^{\prime} \triangleleft_{n}-J_{k}^{\prime}-a l_{\leftarrow} & \text { in case B }
\end{aligned}
$$

for some $a \in \mathbb{N}$; see Definition (6.12). This implies in both cases A and B, analogously to (6.50):

$$
\min J_{k}^{\prime}-\max J_{k-1}^{\prime} \leq \min J_{k}^{\prime}+a l_{\rightarrow}-\max J_{k-1}^{\prime} \leq M
$$

by the maximality of $M$; recall that $M=M_{\rightarrow}$ in case $\mathrm{A}$ and $M=M_{\leftarrow}$ in case $\mathrm{B}$, and that $l_{\rightarrow}=l_{\leftarrow} \in \gamma \mathbb{Z}$ holds in case B; furthermore recall that $J_{k}^{\prime}$ and $J_{k-1}^{\prime}$ belong to different classes modulo $\gamma$. We repeat arguments similar to (6.51):

$$
\begin{aligned}
\min J_{k}^{\prime}-\min J_{0}^{\prime} & =\left(\min J_{k}^{\prime}-\max J_{k-1}^{\prime}\right)+\left(c_{1} n-1\right) l_{\rightarrow}+\left(\min J_{k-1}^{\prime}-\min J_{0}^{\prime}\right) \\
& \leq M+\left(c_{1} n-1\right) l_{\rightarrow}+\left(\min J_{k-1}-\min J_{0}\right)=\min J_{k}-\min J_{0}
\end{aligned}
$$


with equality only if $\left(J_{k}^{\prime}\right)_{j=0, \ldots, k} \in \operatorname{Max} \operatorname{Seq}\left(J, \zeta^{\prime}, k-1\right)$ and $\min J_{k}^{\prime}-\max J_{k-1}^{\prime}=M$. This proves in analogy to $(6.52)$ :

$$
\operatorname{Max} \operatorname{Seq}\left(J, \zeta^{\prime}, k\right)=\left\{\left(J_{j}\right)_{j=0, \ldots, k}\right\} .
$$

Since $\tilde{\iota}_{\gamma}$ is bijective, the facts $T_{I_{j}} I_{j} \subseteq \operatorname{domain}\left(\zeta^{\prime}\right),\left(\zeta^{\prime}\left\lceil T_{I_{j}} I_{j}\right)_{\rightarrow}=\left(w\left\lceil I_{j}\right)_{\rightarrow}\right.\right.$, and $\left(I_{j}\right)_{j} \in \operatorname{Seq}(I, w, k)$ imply

$$
\left(T_{I_{j}} I_{j}\right)_{j} \in \operatorname{Seq}\left(J, \zeta^{\prime}, k\right)
$$

Similarly, $\tilde{T}_{J_{j}} J_{j} \subseteq \operatorname{domain}(w),\left(w\left\lceil\tilde{T}_{J_{j}} J_{j}\right)_{\rightarrow}=\left(\zeta^{\prime}\left\lceil J_{j}\right)_{\rightarrow}\right.\right.$, and $\left(J_{j}\right)_{j} \in \operatorname{Seq}\left(J, \zeta^{\prime}, k\right)$ imply

$$
\left(\tilde{T}_{J_{j}} J_{j}\right)_{j} \in \operatorname{Seq}(I, w, k) .
$$

Now we set $k=\gamma$. Observe that $I_{\gamma}+\gamma \mathbb{Z}=I_{0}+\gamma \mathbb{Z}$ and $J_{\gamma}+\gamma \mathbb{Z}=J_{0}+\gamma \mathbb{Z}$; hence $T_{I_{0}}=T_{I_{\gamma}}$ and $\tilde{T}_{J_{0}}=\tilde{T}_{J_{\gamma}}$. Thus, using (6.52), (6.57), (6.58), (6.59), and the defining property of MaxSeq, we obtain

$$
\begin{aligned}
\min I_{\gamma}-\min I_{0} & =\min T_{I_{\gamma}} I_{\gamma}-\min T_{I_{0}} I_{0} \\
& \leq \min J_{\gamma}-\min J_{0} \\
& =\min \tilde{T}_{J_{\gamma}} J_{\gamma}-\min \tilde{T}_{J_{0}} J_{0} \\
& \leq \min I_{\gamma}-\min I_{0} .
\end{aligned}
$$

Since the first and last term in (6.60) are identical, equality holds everywhere in (6.60). Hence, using (6.57), (6.58), and the defining property of MaxSeq again, we see

$$
\left(T_{I_{j}} I_{j}\right)_{j} \in \operatorname{MaxSeq}\left(J, \zeta^{\prime}, \gamma\right)
$$

and thus $\left(T_{I_{j}} I_{j}\right)_{j}=\left(J_{j}\right)_{j}$, since $\operatorname{MaxSeq}\left(J, \zeta^{\prime}, \gamma\right)$ is a singleton. Furthermore the facts (6.41), (6.42), $\gamma \neq 0$, and $T_{I_{0}}=T_{I_{\gamma}}$ imply $M_{\rightarrow}=M$, since

$$
0=\left(\min I_{\gamma}-\min I_{0}\right)-\left(\min J_{\gamma}-\min J_{0}\right)=M_{\rightarrow} \gamma-M \gamma .
$$

A side remark: consequently case B cannot occur whenever $M_{\rightarrow} \neq M_{\leftarrow}$. Using (6.41) and (6.42) again, we see that all translations $T_{I_{j}}, j=0, \ldots, \gamma$, coincide: $T_{I_{j}}=T_{I}$. We observe

$$
\left(I_{0} \cup \ldots \cup I_{\gamma}\right)+\gamma \mathbb{Z}=I+\left\{j M_{\rightarrow} \mid j=0, \ldots, \gamma\right\}+\gamma \mathbb{Z}=I+\gamma^{\prime} \mathbb{Z} ;
$$

recall that $\gamma^{\prime}$ was defined to be the greatest common divisor of $M_{\rightarrow}$ and $\gamma$. Thus we have shown: the translations $T_{Z}, Z \in \mathbb{Z} / \gamma \mathbb{Z}$, depend only on the rougher modulo class $Z^{\prime}=Z+\gamma^{\prime} \mathbb{Z} \in \mathbb{Z} / \gamma^{\prime} \mathbb{Z}$; hence $T_{Z+\gamma^{\prime} \mathbb{Z}}:=T_{Z}$ and $\iota_{\gamma^{\prime}}: \mathbb{Z} / \gamma^{\prime} \mathbb{Z} \rightarrow \mathbb{Z} / \gamma^{\prime} \mathbb{Z}, \iota_{\gamma^{\prime}}\left(Z^{\prime}\right):=\bigcup_{Z \subseteq Z^{\prime}, Z \in \mathbb{Z} / \gamma \mathbb{Z}} \iota_{\gamma}(Z)$ are well-defined. Since $\iota_{\gamma}: \mathbb{Z} / \gamma \mathbb{Z} \rightarrow \mathbb{Z} / \gamma \mathbb{Z}$ is a bijection, $\iota_{\gamma^{\prime}}$ is a bijection, too. In analogy to $\tilde{\iota}_{\gamma}$, we introduce $\tilde{\iota}_{\gamma^{\prime}}\left(Z^{\prime}\right):= \pm \iota_{\gamma^{\prime}}\left( \pm Z^{\prime}\right)$, ( "+" in case A, "-" in case B). As a consequence of (6.39), we obtain for all $Z^{\prime} \in \mathbb{Z} / \gamma^{\prime} \mathbb{Z}:$

$$
\zeta\left\lceil( \tilde { \iota } _ { \gamma ^ { \prime } } ( Z ^ { \prime } ) \cap [ - 2 ^ { n } , 2 ^ { n } ] ) \subseteq T _ { Z ^ { \prime } } \left[w \lceil Z ^ { \prime } ] \subseteq \zeta \left\lceil\tilde{\iota}_{\gamma^{\prime}}\left(Z^{\prime}\right) .\right.\right.\right.
$$

Hence the event $E_{\bmod \gamma^{\prime} \text { ok }}^{n}$ is valid. This finishes the proof of Lemma 6.28.

Lemma 6.29 $E_{\bmod l \rightarrow \text { ok }}^{n} \cap B_{\text {unique fit }}^{n} \cap E_{\text {neighbor I }}^{n} \cap E_{\text {neighbor II }}^{n} \subseteq E_{\text {mod 1 ok }}^{n}$.

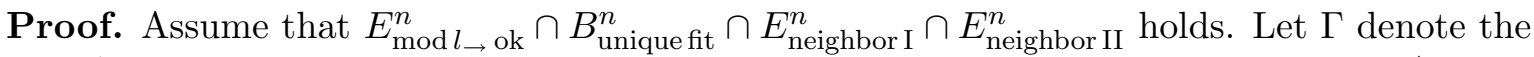
(random) set of all divisors $\gamma \geq 1$ of $l_{\rightarrow}$ for which the event $E_{\bmod \gamma \text { ok }}^{n}$ is valid. $\Gamma \neq \emptyset$, since $E_{\bmod l_{\rightarrow \text { ok }}}^{n}$ holds. The smallest element of $\Gamma$ cannot be bigger than 1 by Lemma 6.28 ; thus it must be equal to 1 . This means that $E_{\bmod 1 \mathrm{ok}}^{n}$ holds. 
Lemma 6.30 For $\gamma=1$, we have $E_{\text {mod } 1 \mathrm{ok}}^{n} \subseteq E_{\text {all pieces ok }}^{n}$.

Proof. This is obvious, since there is only the trivial "modulo class" $Z=\iota_{1}(Z)=\mathbb{Z}$ remaining for $\gamma=1$ : In case $\mathrm{A}$, one has $\xi\left\lceil\left[-2^{n}, 2^{n}\right] \sqsubseteq w \sqsubseteq \xi\left\lceil\left[-9 \cdot 2^{n}, 9 \cdot 2^{n}\right]\right.\right.$, and in case $\mathrm{B}$, one has $\xi\left[\left[-2^{n}, 2^{n}\right] \sqsubseteq w^{\leftrightarrow} \sqsubseteq \xi\left[\left[-9 \cdot 2^{n}, 9 \cdot 2^{n}\right]\right.\right.$.

Theorem 6.31 $B_{\text {seed I }}^{n} \cap B_{\text {unique fit }}^{n} \cap B_{\text {all paths }, \tau}^{n} \cap B_{\text {outside out }}^{n} \cap B_{\text {recogn straight }}^{n} \cap B_{\text {signals }}^{n} \cap E_{\text {stop }, \tau}^{m} \subseteq$ $E_{\text {all pieces ok }}^{n}$

Proof. We collect the results of Lemmas 6.5, 6.10, 6.12, 6.19, 6.21, 6.23, 6.25, 6.27, 6.29, and 6.30 in the following list:

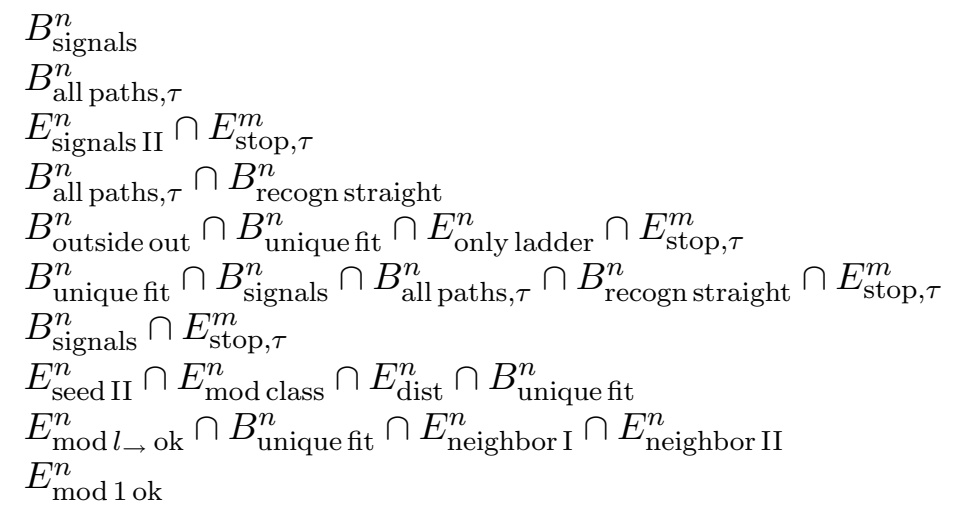

$\subseteq E_{\text {signals II }}^{n}$,
$\subseteq E_{\text {neighbor I }}^{n}$
$\subseteq E_{\text {neighbor II }}^{n}$
$\subseteq E_{\text {only ladder }}^{n}$,
$\subseteq E_{\text {mod class }}^{n}$
$\subseteq E_{\text {seed II }}^{n}$
$\subseteq E_{\text {dist }}^{n}$,
$\subseteq E_{\text {mod } l \rightarrow \text { ok }}^{n}$,
$\subseteq E_{\text {mod 1 ok }}^{n}$,
$\subseteq E_{\text {all pieces ok }}^{n}$.

The claim of the theorem is a simple combination of these inclusions.

\subsection{Probabilistic estimates for basic events}

In this subsection we show that the "basic events" $B_{\ldots}$ occur very probably. Together with the result of the previous subsections this shows that the partial reconstruction algorithms $\operatorname{Alg}^{n}$ yield with high probability a correctly reconstructed piece of scenery.

We start with an elementary auxiliary lemma:

Lemma 6.32 Let $f: I_{0} \rightarrow J$ be a finite injection without fixed points. Then there is $I^{\prime} \subseteq I_{0}$ with $\left|I^{\prime}\right| \geq\left|I_{0}\right| / 3$ and $f\left[I^{\prime}\right] \cap I^{\prime}=\emptyset$.

Proof. We construct recursively finite sequences $\left(I_{k}\right)$ and $\left(I_{k}^{\prime}\right)$, for $k-1<\left|I_{0}\right| / 3$, of subsets of $I_{0}$. The "loop invariants" of the recursion are: $f\left[I_{k}\right] \cap I_{k}^{\prime}=\emptyset, f\left[I_{k}^{\prime}\right] \cap I_{k}=\emptyset, f\left[I_{k}^{\prime}\right] \cap I_{k}^{\prime}=\emptyset$, $I_{k} \cap I_{k}^{\prime}=\emptyset,\left|I_{k}^{\prime}\right|=k$, and $\left|I_{k}\right| \geq\left|I_{0}\right|-3 k$.

The recursion starts with the given $I_{0}$ and with $I_{0}^{\prime}=\emptyset$. In the $(k+1)$ st step, $k<\left|I_{0}\right| / 3$, we choose any point $x \in I_{k}$, and define $I_{k+1}^{\prime}:=I_{k}^{\prime} \cup\{x\}$. If $f^{-1}(x)$ exists, then we set $I_{k+1}:=$ $I_{k} \backslash\left\{x, f(x), f^{-1}(x)\right\}$; else we set $I_{k+1}:=I_{k} \backslash\{x, f(x)\}$.

Note that the validity of the above "loop invariants" is indeed preserved by the recursion; the fact $f(x) \neq x$ is used for the third loop invariant.

Finally we set $I^{\prime}:=I_{k}^{\prime}$ for $k:=\min \left\{j \in \mathbb{N}|3 j \geq| I_{0} \mid\right\}$; then $I^{\prime} \subseteq I_{0}$ is well-defined and fulfills the claims in Lemma 6.32.

Lemma 6.33 There exists constants $c_{18}, c_{19}>0$ not depending on $n$ such that for all finite $\tilde{J} \subset \mathbb{N}$ one has

$$
P\left[\left(B_{\text {unique fit }}^{n}(\tilde{J})\right)^{\mathrm{c}}\right] \leq c_{18} e^{-c_{19} n} .
$$


Proof. Let $i, j \in\left\{1, \ldots, l^{2}\right\}$, and let $I \subseteq\left[-11 \cdot 2^{n}, 11 \cdot 2^{n}\right] \backslash \tilde{J}$ be a $i$-spaced interval, and $J \subseteq\left[-11 \cdot 2^{n}, 11 \cdot 2^{n}\right] \backslash \tilde{J}$ be a $j$-spaced interval with $|I|=|J| \geq 1$. Let $f: I \rightarrow J$ be a monotonically increasing or decreasing bijection, but not the identity map; thus the case $I=J$ can only occur if $f$ is decreasing.

We claim: For some constants $c_{12}>0$ and $c_{13}>2 \log 2 / c_{2}$ (not depending on $i, j, I$, or $J$ ) we have

$$
P\left[\xi \circ f=\xi\lceil I] \leq c_{12} e^{-c_{13}|I|} .\right.
$$

Note that $\xi \circ f=\xi\left\lceil I\right.$ is equivalent to $\left(\xi\lceil J)_{\rightarrow}=\left(\xi\lceil I)_{\rightarrow}\right.\right.$ if $f$ is increasing, and it is equivalent to $\left(\xi\lceil J)_{\rightarrow}=\left(\xi\lceil I)_{\leftarrow}\right.\right.$ if $f$ is decreasing.

Before proving (6.66), let us show how it implies (6.65): There are at most $l^{2}$ choices for $(i, j)$, and given $(i, j)$, there are at most $\left(22 \cdot 2^{n}+1\right)^{2} \leq 500 \cdot 2^{2 n}$ choices for $(I, J)$ with $|I|=|J|=c_{2} n$; finally there is one binary choice: $f$ is increasing or decreasing. If $\xi \circ f \neq \xi\lceil I$ holds for all of these choices (with the trivial exception $I=J$ and $f=\mathrm{id}$ ), then the event $B_{\text {unique fit }}^{n}$ is valid; note that it suffices to consider $|I|=|J|=c_{2} n$ instead of $|I|=|J| \geq c_{2} n$, since it suffices to consider subintervals of $I, J$ consisting only of $c_{2} n$ points. Hence (6.66) implies (6.65):

$$
P\left[\left(B_{\text {unique fit }}^{n}\right)^{\mathrm{c}}\right] \leq l^{2} \cdot 500 \cdot 2^{2 n} \cdot 2 \cdot c_{12} e^{-c_{13} c_{2} n}=c_{18} e^{-c_{19} n},
$$

where $c_{18}:=1000 l^{2} c_{12}$ and $c_{19}:=c_{13} c_{2}-2 \log 2>0$.

We prove (6.66) next: unless $f$ is the identity map, it can have at most a single fixed point, since it is the restriction of some affine-linear map to the ladder interval $I$. Remove this fixed point from $I$, if it exists; call $I_{0}$ the set of all remaining points. By Lemma 6.32, there is $I^{\prime} \subseteq I_{0}$ with $\left|I^{\prime}\right| \geq\left|I_{0}\right| / 3 \geq(|I|-1) / 3$ and $f\left[I^{\prime}\right] \cap I^{\prime}=\emptyset$. Hence $\xi\left\lceil f\left[I^{\prime}\right]\right.$ and $\xi\left\lceil I^{\prime}\right.$ are independent random pieces of scenery; thus

$$
P\left[\xi \circ f=\xi\lceil I] \leq P\left[\xi \circ f \left\lceilI^{\prime}=\xi\left\lceil I^{\prime}\right]=|\mathcal{C}|^{-\left|I^{\prime}\right|} \leq|\mathcal{C}|^{-(|I|-1) / 3} ;\right.\right.\right.
$$

thus (6.66) follows with $c_{13}:=(\log |\mathcal{C}|) / 3$ and $c_{12}:=|\mathcal{C}|^{1 / 3}$. Note that $c_{13} c_{2}-2 \log 2>0$ since $c_{2}$ was required to be large enough; recall Subsection 2.1 .

Lemma 6.34 Let $P^{\prime}$ be a probability distribution on $\Omega$ such that $S$ has the same distribution with respect to both measures $P$ and $P^{\prime}$, and let $\tau$ be a sequence of $\mathcal{G}$-adapted stopping times. Then there exist constants $c_{20}, c_{21}>0$ not depending on $n$ such that:

$$
P^{\prime}\left[\left(B_{\text {all paths }, \tau}^{n}\right)^{\mathrm{c}} \cap E_{\mathrm{stop}, \tau}^{m}\right] \leq c_{21} e^{-c_{20} n} .
$$

We use this lemma twice below: Once for $P^{\prime}=P$ in this section, and in Section 8 for $P^{\prime}=\tilde{P}$, which was defined in (3.5). Note that the following proof does not need any assumptions on the distribution of the scenery $\xi$; knowing the distribution of the random walk $S$ suffices.

Proof of Lemma 6.34. Let $k<2^{\alpha n}$ and $R \in \operatorname{AdPath}\left(12 \cdot 2^{n}, 3 c_{1} n\right)$. We set

$$
\begin{aligned}
B_{R}^{n, k} & :=\left\{\exists j \in\left[0,2^{2 n}-3 c_{1} n\right]: \text { TimeShift }{ }^{\tau(k)+j}(R) \subseteq S\right\}, \\
E_{\mathrm{stop}, \tau, k}^{m} & :=\left\{\begin{array}{l}
\tau_{k}(\chi)<2^{12 \alpha n_{m}},\left|S\left(\tau_{k}(\chi)\right)\right| \leq 2^{n_{m}}, \\
\tau_{j}(\chi)+2 \cdot 2^{2 n_{m}} \leq \tau_{k}(\chi) \text { for } j<k
\end{array}\right\}, \\
A_{R}^{n, k} & :=E_{\mathrm{stop}, \tau, k}^{m} \backslash B_{R}^{n, k} .
\end{aligned}
$$


Note that $B_{\text {all paths }, \tau}^{n}=\bigcap_{R \in \operatorname{AdPath}\left(12 \cdot 2^{n}, 3 c_{1} n\right)} \bigcup_{k=0}^{2^{\alpha n}-1} B_{R}^{n, k}$ and $E_{\text {stop }, \tau}^{m} \subseteq E_{\text {stop }, \tau, k}^{m}$ for $k \leq 2^{\alpha n}$, and thus

$$
E_{\text {stop }, \tau}^{m} \backslash B_{\text {all paths }, \tau}^{n} \subseteq \bigcup_{R \in \operatorname{AdPath}\left(12 \cdot 2^{n}, 3 c_{1} n\right)} \bigcap_{k=0}^{2^{\alpha n}-1} A_{R}^{n, k} .
$$

In the following, $R$ runs over the set $\operatorname{AdPath}\left(12 \cdot 2^{n}, 3 c_{1} n\right)$ :

$$
\begin{aligned}
& P^{\prime}\left[\left(B_{\text {all paths }, \tau}^{n}\right)^{\mathrm{c}} \cap E_{\text {stop }, \tau}^{m}\right] \leq\left|\operatorname{AdPath}\left(12 \cdot 2^{n}, 3 c_{1} n\right)\right| \max _{R} P^{\prime}\left[\bigcap_{k=0}^{2^{\alpha n}-1} A_{R}^{n, k}\right], \\
& \left|\operatorname{AdPath}\left(12 \cdot 2^{n}, 3 c_{1} n\right)\right| \leq 25 \cdot 2^{n}|\mathcal{M}|^{3 c_{1} n}, \\
& P^{\prime}\left[\bigcap_{k=0}^{2^{\alpha n}-1} A_{R}^{n, k}\right]=\prod_{k=0}^{2^{\alpha n}-1} P^{\prime}\left[A_{R}^{n, k} \mid \bigcap_{j<k} A_{R}^{n, j}\right], \\
& P^{\prime}\left[A_{R}^{n, k} \mid \bigcap_{j<k} A_{R}^{n, j}\right] \leq P^{\prime}\left[\left(B_{R}^{n, k}\right)^{\mathrm{c}} \mid E_{\mathrm{stop}, \tau, k}^{m} \cap \bigcap_{j<k} A_{R}^{n, j}\right] ;
\end{aligned}
$$

the last statement follows from the elementary fact $P^{\prime}[A \cap B \mid C] \leq P^{\prime}[A \mid B \cap C]$. We have $C_{R}^{n, k}:=$ $E_{\text {stop }, \tau, k}^{m} \cap \bigcap_{j<k} A_{R}^{n, j} \in \mathcal{F}_{\tau_{k}}$, i.e. one can decide whether the event $C_{R}^{n, k}$ holds by observing $\xi$ and $S(0), \ldots, S\left(\tau_{k}\right)$. Furthermore, if $C_{R}^{n, k}$ holds, then $\left|S\left(\tau_{k}(\chi)\right)\right| \leq 2^{n}$, and as a consequence of the local Central Limit Theorem [5], Theorem 5.2 (page 132) we get: there is a constant $c_{22}>0$ such that for all $x, y$ with $|x| \leq 12 \cdot 2^{n}$ and $|y| \leq 2^{n}: P\left[y+S(j)=x\right.$ for some $\left.j \in\left[0,2^{2 n}-3 c_{1} n\right]\right] \geq$ $c_{22} 2^{-n}$; note that $y+S$ is a random walk starting in the point $y$, and recall that $n=n_{m} \geq n_{0}$; thus $2^{2 n}-3 c_{1} n \geq 2^{2 n} / 2$ holds by our choice of $n_{0}$ in Subsection 2.1. Also, the random walk need not be aperiodic; it suffices that it can reach every integer, i.e. that the greatest common divisor of the elements of $|\mathcal{M}|$ is 1 . Thus by the strong Markov property:

$$
\inf _{|x| \leq 12 \cdot 2^{n}} P^{\prime}\left[S(\tau(k)+j)=x \text { for some } j \in\left[0,2^{2 n}-3 c_{1} n\right] \mid C_{R}^{n, k}\right] \geq c_{22} 2^{-n} .
$$

Once it is in the starting point $x$, the probability that $S$ follows an admissible path $R \in$ $\operatorname{AdPath}\left(12 \cdot 2^{n}, 3 c_{1} n\right)$ for the next $3 c_{1} n-1$ steps is bounded from below by $\mu_{\min }^{3 c_{1} n}$. Here $\mu_{\text {min }}:=\min \{\mu(\{x\}) \mid x \in \mathcal{M}\}$ is the smallest positive probability for a jump. Therefore, using the strong Markov property again:

$$
P^{\prime}\left[B_{R}^{n, k} \mid C_{R}^{n, k}\right] \geq c_{22} 2^{-n} \mu_{\min }^{3 c_{1} n} .
$$

We combine (6.74)-(6.77) and (6.79) to obtain

$$
\begin{aligned}
P^{\prime}\left[E_{\text {stop }, \tau}^{m} \backslash B_{\text {all paths }, \tau}^{n}\right] & \leq 25 \cdot 2^{n}|\mathcal{M}|^{3 c_{1} n}\left(1-c_{22} 2^{-n} \mu_{\min }^{3 c_{1} n}\right)^{2^{\alpha n}} \\
& \leq 25 \cdot 2^{n}|\mathcal{M}|^{3 c_{1} n} \exp \left\{-c_{22} 2^{-n} \mu_{\min }^{3 c_{1} n} 2^{\alpha n}\right\} \\
& \leq 25 \exp \left\{n\left(\log 2+3 c_{1} \log |\mathcal{M}|\right)-c_{22} e^{n\left(\alpha \log 2+3 c_{1} \log \mu_{\min }-\log 2\right)}\right\} .
\end{aligned}
$$

Now $\alpha>1-3 c_{1} \log _{2} \mu_{\text {min }}$ by our choice of $\alpha$ in Subsection 2.1; thus the right hand side of the last inequality converges to 0 superexponentially fast as $n \rightarrow \infty$. Note that we may choose an upper bound $c_{21} e^{-c_{20} n}$ for the right hand side in (6.80), where neither $c_{21}$ nor $c_{20}$ depend on $\alpha$ or $c_{1}$. This is true since $n \geq n_{0}$, and $n_{0}$ was chosen large enough, depending on $c_{1}$ and $\alpha$; recall Subsection 2.1. This proves the lemma. 
Lemma 6.35 There exists a constant $c_{23}>0$ not depending on $n$ such that:

$$
P\left[\left(B_{\text {outside out }}^{n}\right)^{\mathrm{c}}\right] \leq 160 e^{-c_{23} n} .
$$

Proof of Lemma 6.35. The set $\left[-2 \cdot l 2^{2 n}, 2 \cdot l 2^{2 n}\right] \backslash\left[-10 \cdot 2^{n}, 10 \cdot 2^{n}\right]$ contains less than $4 \cdot l \cdot 2^{2 n}$ points, and for every fixed starting point the number of admissible paths with $c_{1} n / 2$ points is equal to $|\mathcal{M}|^{c_{1} n / 2-1}$. Hence there are less than $4 \cdot l 2^{2 n}|\mathcal{M}|^{c_{1} n / 2}$ paths $R \in \operatorname{AdPaths}\left(2 \cdot l 2^{2 n}, c_{1} n / 2\right)$ with $R(i) \notin\left[-10 \cdot 2^{n}, 10 \cdot 2^{n}\right]$ for all $i=0, \ldots, c_{1} n / 2$. On the other hand, there are less than $40 \cdot 2^{n}$ ladder words of length $c_{1} n / 2$ in $\left[-9 \cdot 2^{n}, 9 \cdot 2^{n}\right]$. The colors $\xi \circ R$ that a path in $R \in \operatorname{AdPaths}\left(2^{2 n}, c_{1} n / 2\right)$ with $R(i) \notin\left[-10 \cdot 2^{n}, 10 \cdot 2^{n}\right]$ for all $i=0, \ldots, c_{1} n / 2-1$ reads are independent of the colors inside $\left[-9 \cdot 2^{n}, 9 \cdot 2^{n}\right]$. Thus the probability that a given path $R \in \operatorname{AdPaths}\left(2^{2 n}, c_{1} n / 2\right)$ with $R(i) \notin\left[-10 \cdot 2^{n}, 10 \cdot 2^{n}\right]$ for all $i=0, \ldots, c_{1} n / 2-1$ reads the same colors as a fixed ladder word in $\left[-9 \cdot 2^{n}, 9 \cdot 2^{n}\right]$ is $|\mathcal{C}|^{-c_{1} n / 2}$. Thus

$$
P\left[\left(B_{\text {outside out }}^{n}\right)^{\mathrm{c}}\right] \leq 160 l 2^{3 n}|\mathcal{M}|^{c_{1} n / 2}|\mathcal{C}|^{-c_{1} n / 2} .
$$

Since $|\mathcal{M}|<|\mathcal{C}|$, the last expression becomes exponentially decreasing in $n$ since $c_{1}>6 / \log \frac{|\mathcal{C}|}{|\mathcal{M}|}$ since $c_{1}$ was chosen large enough; see Subsection 2.1. This proves the lemma.

We prepare the treatment of the event $B_{\text {recognstraight }}^{n}(\tilde{J})$ by the following combinatoric lemma:

Lemma 6.36 Let $c_{24}:=1 /\left(2|\mathcal{M}|\left(l_{\rightarrow}+l_{\leftarrow}\right)\right)$. There are two intervals $I_{1}, I_{2} \subseteq\left[0, c_{1} n[\right.$ with $\left|I_{1}\right|=\left|I_{2}\right| \geq c_{24} c_{1} n-1$ such that the following statement is valid: For all $R \in \operatorname{AdPaths}\left(11 \cdot 2^{n}, c_{1} n\right)$ with $R\left(c_{1} n-1\right)-R(0) \notin\left\{\left(c_{1} n-1\right) l_{\rightarrow},-\left(c_{1} n-1\right) l_{\leftarrow}\right\}$, there is $I \in\left\{I_{1}, I_{2}\right\}$ and an admissible path $\bar{R} \in \operatorname{AdPaths}\left(12 \cdot 2^{n}, c_{1} n\right)$ with the following properties:

- $R(0)=\bar{R}(0), \quad R\left(c_{1} n-1\right)=\bar{R}\left(c_{1} n-1\right)$.

- At least one of the following holds:

1. for all $(i, j) \in I \times I$ with $j<i: \bar{R}(i) \notin\{R(j), \bar{R}(j)\}$;

2. for all $(i, j) \in I \times I$ with $i<j: \bar{R}(i) \notin\{R(j), \bar{R}(j)\}$.

Proof. We define $k:=\left\lfloor c_{24} c_{1} n\right\rfloor, I^{\prime}:=[1,2 k] \subseteq\left[0, c_{1} n\left[, I_{1}:=[1, k]\right.\right.$, and $I_{2}:=[k+1,2 k]$. We observe $\left|I_{1}\right|,\left|I_{2}\right| \geq c_{24} c_{1} n-1$ and $I_{1}, I_{2} \subseteq\left[0, c_{1} n[\right.$.

Let $R \in \operatorname{AdPaths}\left(11 \cdot 2^{n}, c_{1} n\right)$ be not a ladder path. We show first: There are $R^{\prime}, R^{\prime \prime} \in$ $\operatorname{AdPaths}\left(12 \cdot 2^{n}, c_{1} n\right)$ such that $R^{\prime}(0)=R^{\prime \prime}(0)=R(0), R^{\prime}\left(c_{1} n-1\right)=R^{\prime \prime}\left(c_{1} n-1\right)=R\left(c_{1} n-1\right)$, $R^{\prime}\left\lceil I^{\prime}\right.$ and $R^{\prime \prime}\left\lceil I^{\prime}\right.$ are ladder paths, and $R^{\prime \prime}\left\lceil I^{\prime}=r+R^{\prime}\left\lceil I^{\prime}\right.\right.$ for some $r \neq 0$, i.e. $R^{\prime \prime}\left\lceil I^{\prime}\right.$ is obtained from $R^{\prime}\left\lceil I^{\prime}\right.$ by a spatial translation.

To prove this claim, let $d=\left(d_{i}\right)_{i=1 \ldots, c_{1} n-1} \in \mathcal{M}^{c_{1} n-1}, d_{i}:=R(i)-R(i-1)$, be the jump sizes in $R$. Every other $\tilde{d} \in \mathcal{M}^{c_{1} n-1}$ with $\sum_{i=1}^{c_{1} n-1} \tilde{d}_{i}=\sum_{i=1}^{c_{1} n-1} d_{i}$ gives rise to an admissible path $\tilde{R} \in \operatorname{AdPaths}\left(12 \cdot 2^{n}, c_{1} n\right)$, too, with $\tilde{R}(0)=R(0), \tilde{R}\left(c_{1} n-1\right)=R\left(c_{1} n-1\right)$, and with jump sizes $\tilde{d}_{i}=\tilde{R}(i)-\tilde{R}(i-1)$; namely $\tilde{R}(i):=R(0)+\sum_{j=1}^{i} \tilde{d}_{j}$. Since $\tilde{R}$ has its starting point and end point in $\left[-11 \cdot 2^{n}, 11 \cdot 2^{n}\right]$ and since $c_{1} n l<2^{n}$, the path $\tilde{R}$ can indeed not leave the range $\left[-12 \cdot 2^{n}, 12 \cdot 2^{n}\right]$.

There are at most $|\mathcal{M}|$ possible values for $d_{i}$, but there are $c_{1} n$ possible indices $i$; thus at least one value $a \in \mathcal{M}$ occurs in the $d_{i}$ at least $c_{1} n /|\mathcal{M}|$ times. We choose $2 k\left(a+l_{\leftarrow}\right) \geq 0$ indices $i$ with $d_{i}=a$ and replace them by $l_{\rightarrow}$, and we choose $2 k\left(l_{\rightarrow}-a\right) \geq 0$ different indices $i$ with $d_{i}=a$ and replace them by $-l_{\leftarrow ;}$ note that $2 k\left(a+l_{\leftarrow}\right)+2 k\left(l_{\rightarrow}-a\right)=2 k\left(l_{\rightarrow}+l_{\leftarrow}\right) \leq c_{1} n /|\mathcal{M}|$. 
We end up with a new vector $\tilde{d} \in \mathcal{M}^{c_{1} n-1}$ with $\sum_{i=1}^{c_{1} n-1} \tilde{d}_{i}=\sum_{i=1}^{c_{1} n-1} d_{i}$, since $2 k\left(l_{\leftarrow}+l_{\rightarrow}\right) a=$ $2 k\left(a+l_{\leftarrow}\right) l_{\rightarrow}+2 k\left(l_{\rightarrow}-a\right)\left(-l_{\leftarrow}\right) . \tilde{d}$ contains at least $2 k$ entries with value $l_{\rightarrow}$, or it contains at least $2 k$ entries with value $-l_{\leftarrow}$, since already the described replacement procedure has produced sufficiently many such entries. However, not all entries of $\tilde{d}$ can equal $l_{\rightarrow}$; similarly not all its entries can equal $-l_{\leftarrow}$, since $R$ is not a ladder path. We permute the entries of $\tilde{d}_{i}$ in two different ways; the resulting vectors are called $d^{\prime}$ and $d^{\prime \prime}$ : First to obtain $d^{\prime}$, permute the entries in $\tilde{d}$ such that the first $2 k$ permuted entries $d_{i}^{\prime}, i=1, \ldots, 2 k$ either all equal $l_{\rightarrow}$ or all equal $-l_{\leftarrow}$; the order of the remaining entries is irrelevant. Second to obtain $d^{\prime \prime}$, transpose the first entry $d_{1}^{\prime}$ with a different entry $d_{i}^{\prime} \neq d_{1}^{\prime}$. Let $R^{\prime}$ and $R^{\prime \prime}$ be admissible pieces of paths with $R^{\prime}(0)=R^{\prime \prime}(0)=R(0)$ and step sizes $d_{i}^{\prime}=R^{\prime}(i)-R^{\prime}(i-1)$ and $d_{i}^{\prime \prime}=R^{\prime \prime}(i)-R^{\prime \prime}(i-1)$, respectively. Recall $I^{\prime}=[1,2 k]$; then $R^{\prime}\left\lceil I^{\prime}\right.$ and $R^{\prime \prime}\left\lceil I^{\prime}\right.$ are ladder paths, and $R^{\prime \prime}\left\lceil I^{\prime}\right.$ is obtained from translating $R^{\prime}\left\lceil I^{\prime}\right.$ by $r:=d_{1}^{\prime \prime}-d_{1}^{\prime} \neq 0$. Thus our first claim holds.

$R^{\prime}\left\lceil I^{\prime}\right.$ is a right ladder path or a left ladder path. Without loss of generality, we assume that it is a right ladder path; the case of left ladder paths can be treated similarly by reversing directions in the arguments below. Furthermore, we assume without loss of generality $r>0$; otherwise we exchange $R^{\prime}$ with $R^{\prime \prime}$.

We are ready to prove the claim of the lemma; recall that $k$ is a point in the middle of $I^{\prime}$. There are two cases:

- If $R(k)>R^{\prime}(k)$, then we take $I:=I_{1}$ and $\bar{R}:=R^{\prime}$. Since $R^{\prime}\lceil I$ is a right ladder path, it moves with maximal speed $l_{\rightarrow}$ to the right. $R$ cannot move faster than that to the right; thus $R(j)>R^{\prime}(i)$ and $R^{\prime}(j)>R^{\prime}(i)$ for all $i, j \in I$ with $i<j$.

- If $R(k) \leq R^{\prime}(k)$, then $R(k)<r+R^{\prime}(k)=R^{\prime \prime}(k)$; this time we take $I:=I_{2}$ and $\bar{R}:=R^{\prime \prime}$. The same argument as above yields $R(j)<R^{\prime \prime}(i)$ and $R^{\prime \prime}(j)<R^{\prime \prime}(i)$ for all $i, j \in I$ with $j<i$.

This proves Lemma 6.36.

Lemma 6.37 There exist positive constants $c_{25}$ and $c_{26}$ not depending on $n$ such that for all finite $\tilde{J} \subset \mathbb{N}$ one has

$$
P\left[\left(B_{\text {recogn straight }}^{n}(\tilde{J})\right)^{\mathrm{c}}\right] \leq c_{25} e^{-c_{26} n} .
$$

Proof of Lemma 6.37. Let $\operatorname{AdPaths}_{n}^{\sim}$ denote the set of all $R \in \operatorname{AdPaths}\left(11 \cdot 2^{n}, c_{1} n\right)$ with $R\left(c_{1} n\right)-R(0) \notin\left\{\left(c_{1} n-1\right) l_{\rightarrow},-\left(c_{1} n-1\right) l_{\leftarrow}\right\}$. (Roughly speaking, AdPaths $\tilde{n}$ consists of "nonstraight" paths.) Given $R \in$ AdPaths $_{n}^{\sim}$, we take $I=I(R) \subseteq\left[0, c_{1} n\left[\right.\right.$ and $\bar{R} \in \operatorname{AdPaths}\left(12 \cdot 2^{n}, c_{1} n\right)$ as in Lemma 6.36. Without loss of generality assume that condition 1. in Lemma 6.36 is satisfied. We prove for all $I^{\prime} \subseteq I$ by induction on $\left|I^{\prime}\right|$ :

$$
P\left[( \xi \circ R ) \left\lceilI^{\prime}=(\xi \circ \bar{R})\left\lceil I^{\prime}\right]=|\mathcal{C}|^{-\left|I^{\prime}\right|} .\right.\right.
$$

This is obvious for $I^{\prime}=\emptyset$. For other $I^{\prime}$, let $I^{\prime \prime}:=I^{\prime} \backslash \max I^{\prime}$. Then $\xi\left(\bar{R}\left(\max I^{\prime}\right)\right)$ is independent of $\left(\xi \circ R\left\lceil I^{\prime \prime}, \xi \circ \bar{R}\left\lceil I^{\prime \prime}\right)\right.\right.$, since they are generated by disjoint parts of the scenery. Thus

$$
\begin{aligned}
P\left[( \xi \circ R ) \left\lceilI^{\prime}=(\xi \circ \bar{R})\left\lceil I^{\prime}\right]\right.\right. & =P\left[\xi\left(R\left(\max I^{\prime}\right)\right)=\xi\left(\bar{R}\left(\max I^{\prime}\right)\right)\right] \cdot P\left[( \xi \circ R ) \left\lceilI^{\prime \prime}=(\xi \circ \bar{R})\left\lceil I^{\prime \prime}\right]\right.\right. \\
& =|\mathcal{C}|^{-1}|\mathcal{C}|^{-\left|I^{\prime \prime}\right|}=|\mathcal{C}|^{-\left|I^{\prime}\right|} .
\end{aligned}
$$

By taking $I^{\prime}=I$, we conclude $P\left[(\xi \circ R)\left\lceil I=(\xi \circ \bar{R})\lceil I] \leq|\mathcal{C}|^{-|I|}\right.\right.$. 
It does not suffice to multiply the last bound with the bound $23 \cdot 2^{n}|\mathcal{M}|^{c_{1} n} \geq \mid \operatorname{AdPaths}(11$. $\left.2^{n}, c_{1} n\right) \mid$ : the product may sometimes be bigger than 1 .

To overcome this difficulty, we partition $\operatorname{AdPaths}_{n}^{\sim} \ni R$ into equivalence classes $[R]$ : we put two paths into the same class if and only if they are mapped to the same value by the map $R \mapsto\left(R(0), R\left(c_{1} n-1\right), I(R), R\lceil I(R))\right.$; here $I(R) \in\left\{I_{1}, I_{2}\right\}$ is taken from Lemma 6.36. We bound the number of equivalence classes from above: For our purposes, a simple but rough bound suffices: There are at most $25 \cdot 2^{n}$ choices for each of $R(0), R\left(c_{1} n-1\right)$, and $R(\min I(R))$, and there is a binary choice $I(R) \in\left\{I_{1}, I_{2}\right\}$; finally given $R(\min I(R))$, there are not more than $|\mathcal{M}|^{k}$ choices for $R\left\lceil I(R)\right.$, where again $k=\left\lfloor c_{24} c_{1} n\right\rfloor=|I(R)|$. Altogether the number of equivalence classes is bounded by $c_{27} 2^{3 n}|\mathcal{M}|^{k}$, where $c_{27}:=2 \cdot 25^{3}$. We may choose a map $\operatorname{AdPaths}_{n}^{\sim} \rightarrow \operatorname{AdPaths}\left(12 \cdot 2^{n}, c_{1} n\right), R \mapsto \bar{R}$ such that $\bar{R}$ depends only of the equivalence class $[R]$ and fulfills the claim in Lemma 6.36 .

We observe

$$
\left(B_{\text {recogn straight }}^{n}(\tilde{J})\right)^{\mathrm{c}} \subseteq\left(B_{\text {recogn straight }}^{n}\right)^{\mathrm{c}} \subseteq\left\{\exists R \in \operatorname{AdPaths}_{n}^{\sim}: \xi \circ R\lceil I(R)=\xi \circ \bar{R}\lceil I(R)\}\right.
$$

recall the definition $(6.21)$ of the event $B_{\text {recogn straight }}^{n}(\tilde{J})$. Thus we get

$$
\begin{aligned}
& P\left[\left(B_{\text {recogn straight }}^{n}(\tilde{J})\right)^{\mathrm{c}}\right] \leq P\left[\exists R \in \operatorname{AdPaths}_{n}^{\sim}: \xi \circ R\lceil I(R)=\xi \circ \bar{R}\lceil I(R)]\right. \\
& \leq \sum_{[R]} P[\xi \circ R\lceil I(R)=\xi \circ \bar{R}\lceil I(R)] \\
& \leq c_{27} 2^{3 n}(|\mathcal{M}| /|\mathcal{C}|)^{k} \leq c_{27}(|\mathcal{C}| /|\mathcal{M}|) \exp \left\{\left(3 \log 2-c_{24} c_{1} \log (|\mathcal{C}| /|\mathcal{M}|)\right) n\right\}
\end{aligned}
$$

We emphasize: the sum in the last but one expression runs over equivalence classes $[R]$, not over paths $R$; the event $\{\xi \circ R\lceil I(R)=\xi \circ \bar{R}\lceil I(R)\}$ does not depend on the choice of $R \in[R]$. We have $c_{24} c_{1} \log (|\mathcal{C}| /|\mathcal{M}|)-3 \log 2 \geq 1$; recall from Subsection 2.1 that $c_{1}$ is large enough. The estimate (6.87) proves the lemma with $c_{26}=1, c_{25}=c_{27}|\mathcal{C}| /|\mathcal{M}|$.

Lemma 6.38 There exist constants $c_{28}>0, c_{29}>0$ such that:

$$
P\left[\left(B_{\text {signals }}^{n}\right)^{\mathrm{c}}\right] \leq c_{29} e^{-c_{28} n} .
$$

Proof of Lemma 6.38. We show that

$$
P\left[B_{\text {sig rr }}^{n}\right] \geq 1-c_{30} e^{-c_{28} n}
$$

for some constants $c_{30}>0$ and $c_{28}>0$. The proof for $B_{\text {sig rl }}^{n}, B_{\text {sig lr }}^{n}$, and $B_{\text {sig ll }}^{n}$ can be done analogously. Take a right ladder path $\pi \in\left[-2 \cdot l 2^{2 n}, 2 \cdot l 2^{2 n}\right]^{\left[0, c_{1} n / 2[\right.}$ and an admissible piece of path $\pi^{\prime} \in \operatorname{AdPath}\left(2 \cdot l 2^{2 n}, c_{1} n / 2\right)$ with $\pi(0)>\pi^{\prime}(0)$. We show by induction over $j \in\left[0, c_{1} n / 2[\right.$ with the abbreviation $I=\left[0, j+1\left[\right.\right.$ and $I^{\prime}=[0, j[$ :

$$
P\left[\xi \circ \pi \left\lceilI^{\prime}=\xi \circ \pi^{\prime}\left\lceil I^{\prime}\right]=|\mathcal{C}|^{-j} .\right.\right.
$$

Indeed, (6.90) is trivial for $j=0$. For the step $j \mapsto j+1$, we observe that $\pi(j)$ is right of all $\pi(i)$ and $\pi^{\prime}(i), i<j$, since $\pi$ is a right ladder path and $\pi(0)>\pi^{\prime}(0)$. Thus $\xi \circ \pi(j)$ is independent of the family $\left(\xi \circ \pi\left\lceil I^{\prime}, \xi \circ \pi^{\prime}\left\lceil I^{\prime}\right)\right.\right.$. Therefore, using our induction hypothesis,

$$
\begin{aligned}
& P\left[\xi \circ \pi \left\lceilI=\xi \circ \pi^{\prime}\lceil I]\right.\right. \\
& \quad=P\left[\xi \circ \pi \left\lceilI^{\prime}=\xi \circ \pi^{\prime}\left\lceil I^{\prime}\right] \cdot P\left[\xi \circ \pi(j)=\xi \circ \pi^{\prime}(j)\right]=|\mathcal{C}|^{-j-1} .\right.\right.
\end{aligned}
$$


For $j=c_{1} n / 2$ we obtain that

$$
P\left[\xi \circ \pi \left\lceil\left[0, c_{1} n / 2\left[=\xi \circ \pi^{\prime}\left\lceil\left[ 0, c_{1} n / 2[] \leq|\mathcal{C}|^{-c_{1} n / 2} .\right.\right.\right.\right.\right.\right.
$$

There are not more than $4 \cdot l 2^{2 n}+1 \leq 5 \cdot l 2^{2 n}$ such $\pi$ and not more than $5 \cdot l 2^{2 n}|\mathcal{M}|^{c_{1} n / 2}$ such $\pi^{\prime}$. Therefore

$$
P\left[\left(B_{\text {sig r }}^{n}\right)^{\mathrm{c}}\right] \leq\left(5 \cdot l 2^{2 n}\right)^{2}|\mathcal{M}|^{c_{1} n / 2}|\mathcal{C}|^{-c_{1} n / 2}
$$

holds; consequently (6.89) is valid with $c_{29}=25 \cdot l^{2}$ and $c_{28}:=1 \leq c_{1} \log (|\mathcal{C}| /|\mathcal{M}|) / 2-4 \log 2$. The last inequality holds, since $|\mathcal{C}|>|\mathcal{M}|$ and $c_{1}$ was chosen large enough; see Subsection 2.1.

Lemma 6.39 There exist constants $c_{31}>0$ and $c_{32}>0$ such that:

$$
P\left[\left(B_{\text {seed I }}^{n}\right)^{\mathrm{c}} \cap E_{\text {stop }, \tau}^{m}\right] \leq c_{32} e^{-c_{31} n} .
$$

Proof. We proceed similarly to the proof of Lemma 6.34. In the following, $Z$ runs over all classes $Z \in \mathbb{Z} / l_{\rightarrow} \mathbb{Z}$. We set for all $Z$ (compare with Definition (6.17) of $B_{\text {seed I }}^{n}$ ):

$$
\begin{aligned}
B_{Z}^{n, k} & :=\left\{\begin{array}{l}
S(\tau(k)+h) \in Z, S\left\lceil\left(\tau(k)+h+\left[0,3 c_{1} n l_{\leftarrow}\right]\right)\right. \text { is a right ladder path, } \\
\text { and } S\left\lceil\left(\tau(k)+h+3 c_{1} n l_{\leftarrow}+\left[0,3 c_{1} n l_{\rightarrow}\right]\right)\right. \text { is a left ladder path. }
\end{array}\right\}, \\
A_{Z}^{n, k} & :=E_{\text {stop }, \tau, k}^{m} \backslash B_{Z}^{n, k}
\end{aligned}
$$

where $E_{\text {stop }, \tau, k}^{m}$ is given by (6.71). Note that $B_{\text {seed I }}^{n}=\bigcap_{Z} \bigcup_{k=0}^{2^{\alpha n}-1} B_{Z}^{n, k}$ and still $E_{\text {stop }, \tau}^{m} \subseteq E_{\text {stop }, \tau, k}^{m}$ for $k<2^{\alpha n}$; thus

$$
E_{\text {stop }, \tau}^{m} \backslash B_{\text {seed I }}^{n} \subseteq \bigcup_{Z}^{2^{\alpha n}-1} \bigcap_{k=0}^{n, k} A_{Z} .
$$

We obtain

$$
\begin{aligned}
& P\left[E_{\mathrm{stop}, \tau}^{m} \backslash B_{\mathrm{seed}}^{n}\right] \leq l_{\rightarrow} \max _{Z} P\left[\bigcap_{k=0}^{2^{\alpha n}-1} A_{Z}^{n, k}\right]=l_{\rightarrow} \max _{Z} \prod_{k=0}^{2^{\alpha n}-1} P\left[A_{Z}^{n, k} \mid \bigcap_{j<k} A_{Z}^{n, j}\right] \\
& P\left[A_{Z}^{n, k} \mid \bigcap_{j<k} A_{Z}^{n, j}\right] \leq P\left[\left(B_{Z}^{n, k}\right)^{\mathrm{c}} \mid E_{\mathrm{stop}, \tau, k}^{m} \cap \bigcap_{j<k} A_{Z}^{n, j}\right] .
\end{aligned}
$$

Since $h+3 c_{1} n l_{\leftarrow}+3 c_{1} n l_{\rightarrow}<2 \cdot 2^{2 n}$, we have $C_{Z}^{n, k}:=E_{\text {stop }, \tau, k}^{m} \cap \bigcap_{j<k} A_{Z}^{n, j} \in \mathcal{F}_{\tau(k)}$. Using Lemma 6.11, we know $l_{\rightarrow} \mathbb{Z}+\operatorname{supp} \mu^{h}=\mathbb{Z}$; hence $c_{33}:=\inf _{x \in \mathbb{Z}} P[x+S(h) \in Z]>0$; (note that the random walk $x+S$ starts in the point $x$ ). Moreover, given that $S(h) \in Z$, the probability to follow a right ladder path in $Z$ in the subsequent $3 c_{1} n l_{\leftarrow}$ steps is $\mu\left(\left\{l_{\rightarrow}\right\}\right)^{c_{1} n l_{\leftarrow}}$, and the probability to follow then a left ladder path in the next $3 c_{1} n l_{\rightarrow}$ steps is $\mu\left(\left\{-l_{\leftarrow}\right\}\right)^{c_{1} n l_{\rightarrow}}$.

Thus by the strong Markov property:

$$
P\left[B_{Z}^{n, k} \mid C_{Z}^{n, k}\right] \geq c_{33} \mu\left(\left\{l_{\rightarrow}\right\}\right)^{3 c_{1} n l_{\leftarrow}} \mu\left(\left\{-l_{\leftarrow}\right\}\right)^{3 c_{1} n l_{\rightarrow}}=c_{33} e^{-c_{34} c_{1} n},
$$

where $c_{34}:=-3 l_{\leftarrow} \log \mu\left(\left\{l_{\rightarrow}\right\}\right)-3 l_{\rightarrow} \log \mu\left(\left\{-l_{\leftarrow}\right\}\right)$. We combine (6.98), (6.99) and (6.100) to obtain

$$
P\left[E_{\text {stop }, \tau}^{m} \backslash B_{\text {seed I }}^{n}\right] \leq l_{\rightarrow}\left(1-c_{33} e^{-c_{34} c_{1} n}\right)^{2^{\alpha n}} \leq l_{\rightarrow} \exp \left\{-c_{33} e^{-c_{34} c_{1} n} 2^{\alpha n}\right\} .
$$


We have $c_{34} c_{1}<\alpha \log 2$, since $\alpha$ was chosen large enough; see Subsection 2.1. Thus the right hand side of the last inequality converges to 0 superexponentially fast as $n \rightarrow \infty$. This proves the lemma, since $n \geq n_{0}$ and $n_{0}$ was chosen large enough.

Finally we reap the results of this section:

Proof of Theorem 6.3. By Theorems 6.17 and 6.31 we have

$$
\begin{aligned}
& E_{\text {xi does it }}^{n} \cap E_{\text {all pieces ok }}^{n} \supseteq \\
& B_{\text {seed I }}^{n} \cap B_{\text {unique fit }}^{n} \cap B_{\text {all paths }}^{n} \cap B_{\text {outside out }}^{n} \cap B_{\text {recogn straight }}^{n} \cap B_{\text {signals }}^{n} \cap E_{\text {stop }, \tau}^{m},
\end{aligned}
$$

hence

$$
\begin{aligned}
& E_{\text {stop }, \tau}^{m} \backslash\left(E_{\text {xi does it }}^{n} \cap E_{\text {all pieces ok }}^{n}\right) \subseteq\left(B_{\text {unique fit }}^{n}\right)^{\mathrm{c}} \cup\left(\left(B_{\text {all paths }}^{n}\right)^{\mathrm{c}} \cap E_{\text {stop }, \tau}^{m}\right) \\
& \cup\left(B_{\text {outside out }}^{n}\right)^{\mathrm{c}} \cup\left(B_{\text {recogn straight }}^{n}\right)^{\mathrm{c}} \cup\left(B_{\text {signals }}^{n}\right)^{\mathrm{c}} \cup\left(\left(B_{\text {seed I }}^{n}\right)^{\mathrm{c}} \cap E_{\text {stop }, \tau}^{m}\right) .
\end{aligned}
$$

Thus Theorem 6.3 follows from the main Lemmas 6.33, 6.34, 6.35, 6.37, 6.38, and 6.39 of this subsection.

\section{How to find back: Correctness of the stopping times $T_{f}$}

In this section, we prove Theorem 3.9.

Definition 7.1 Let $T=\left(T_{k}\right)_{k \in \mathbb{N}}$ be a sequence of $\mathcal{G}$-adapted stopping times. We define the events

$$
\begin{aligned}
E_{\text {no error }, T}^{m} & :=\left\{\forall k \geq 0: \text { if } T_{k}(\chi)<2^{12 \alpha n_{m}}, \text { then }\left|S\left(T_{k}(\chi)\right)\right| \leq 2^{n_{m}}\right\}, \\
E_{\text {enough back }}^{m} & :=\left\{\begin{array}{l}
\text { Up to time } 2^{12 \alpha n_{m}} / 8, S \text { visits } 0 \text { at least } \\
2^{3 \alpha n_{m}} \text { times }
\end{array}\right\} .
\end{aligned}
$$

We abbreviate

$$
\Xi_{\text {reconst }, f}^{m}:=\left\{\xi \in \mathcal{C}^{\mathbb{Z}} \mid P\left[E_{\text {reconst }, f}^{m} \mid \xi\right] \geq \frac{1}{2}\right\} ;
$$

recall Definition (3.15) of the event $E_{\text {reconst,f }}^{m}$.

Lemma 7.2 For some constant $c_{35}$ and all $m \geq 0$ :

$$
1-P\left[E_{\text {enough back }}^{m}\right] \leq c_{35} 2^{-\alpha n_{m}} .
$$

Proof of Lemma 7.2. Let $\left(X_{i}\right)_{i \geq 1}$ denote the time difference between the $(i+1)$ st and the $i$-th visit of $S$ at the origin. By recurrence, $\left(X_{i}\right)_{i \geq 1}$ is a.s. well defined, and by the strong Markov property it is i.i.d. with respect to $P$. Since $S$ starts in the origin, $X_{1}$ is the first return time to the origin, and $\sum_{i=1}^{j} X_{i}$ is (a.s.) the time of the $j$-th visit at the origin. For the sake of this proof, we abbreviate: $x=2^{12 \alpha n_{m}} / 8$ and $y=2^{3 \alpha n_{m}}$. Using

$$
\left(E_{\text {enough back }}^{m}\right)^{\mathrm{c}}=\left\{\sum_{i=1}^{y} X_{i} \geq x\right\} \subseteq\left\{\left(\sum_{i=1}^{y} X_{i}^{1 / 3}\right)^{3} \geq x\right\}
$$


and the Chebyshev-Markov inequality, we obtain the claim (7.4):

$$
\begin{aligned}
1-P\left[E_{\text {enough back }}^{m}\right] & \leq P\left[\sum_{i=1}^{y} X_{i}^{1 / 3} \geq x^{1 / 3}\right] \leq x^{-1 / 3} E\left[\sum_{i=1}^{y} X_{i}^{1 / 3}\right] \\
& =x^{-1 / 3} y E\left[X_{1}^{1 / 3}\right]=2 E\left[X_{1}^{1 / 3}\right] 2^{-\alpha n_{m}} .
\end{aligned}
$$

The fact $E\left[X_{1}^{1 / 3}\right]<\infty$ is an immediate consequence of a lemma proved on page 382 of [24]. In our context, this lemma states that there exists a constant $c_{58}>0$ such that $P[S(k) \neq 0 ; k=$ $1,2, \ldots, n] \leq c_{58} n^{-1 / 2}$ for all $n>0$.

Definition 7.3 Let $v(k), k \geq 0$, denote the $(k+1)$ st visit of $S$ to the origin. We introduce a random set $\mathbb{T}_{f}^{\prime}(\xi, \chi)$ and an event $E_{\text {when back recog }}^{m+1}$ :

$$
\begin{aligned}
& \mathbb{T}_{f}^{\prime}(\xi, \chi):=\left\{t \in \mathbb{N} \mid \xi\left\lceil\left[-2^{n_{m}}, 2^{n_{m}}\right] \preccurlyeq f\left(\theta^{t}(\chi)\right) \preccurlyeq \xi\left\lceil\left[-9 \cdot 2^{n_{m}}, 9 \cdot 2^{n_{m}}\right]\right\}\right.\right. \\
& E_{\text {when back recog, } f}^{m+1}:=\left\{\begin{array}{l}
\text { For more than } 1 / 4 \text { of the points } k \in\left[0,2^{2 \alpha n_{m+1}}[\text { holds }\right. \\
v\left(k 2^{\alpha n_{m+1}}\right) \in \mathbb{T}_{f}^{\prime}(\xi, \chi)
\end{array}\right\} .
\end{aligned}
$$

Lemma 7.4 If the event $E_{\text {reconst }, f}^{m}$ holds, then $\mathbb{T}_{f}(\chi) \supseteq \mathbb{T}_{f}^{\prime}(\xi, \chi) \cap\left[0,2^{12 \alpha n_{m+1}}-2 \cdot 2^{12 \alpha n_{m}}[\right.$.

Proof. We know $\xi\left\lceil\left[-2^{n_{m}}, 2^{n_{m}}\right] \preccurlyeq f(\chi)\right.$ by $E_{\text {reconst }, f}^{m}$. Let $t \in \mathbb{T}_{f}^{\prime}(\xi, \chi), t<2^{12 \alpha n_{m+1}}-2 \cdot 2^{12 \alpha n_{m}}$. Then we also have $\xi\left\lceil\left[-2^{n_{m}}, 2^{n_{m}}\right] \preccurlyeq f\left(\theta^{t} \chi\right)\right.$. Hence $t \in \mathbb{T}_{f}(\chi)$; to this end recall Definition (3.12) of the random set $\mathbb{T}_{f}(\chi)$. This implies the lemma.

Lemma 7.5 Assume that the events $E_{\text {no error }, T_{f}}^{m+1} \cap E_{\text {enough back }}^{m+1} \cap E_{\text {when back recog, } f}^{m+1}$ and $\mathbb{T}_{f}(\chi) \supseteq$ $\mathbb{T}_{f}^{\prime}(\xi, \chi) \cap\left[0,2^{12 \alpha n_{m+1}}-2 \cdot 2^{12 \alpha n_{m}}\left[\right.\right.$ hold. Then $E_{\mathrm{stop}, T_{f}}^{m+1}$ holds, too.

Proof. Using $E_{\text {enough back, }}^{m+1}$ we know

$$
v\left(k 2^{\alpha n_{m+1}}\right) \in\left[0,2^{12 \alpha n_{m+1}} / 8\right] \subseteq\left[0,2^{12 \alpha n_{m+1}}-2 \cdot 2^{12 \alpha n_{m}}[\right.
$$

for all $k \in\left[0,2^{2 \alpha n_{m+1}}\left[\right.\right.$. Since the event $E_{\text {when back recog, } f}^{m+1}$ holds, we obtain $\left|\mathbb{T}_{f}(\chi)\right| \geq \mid \mathbb{T}_{f}^{\prime}(\xi, \chi) \cap$ $\left[0,2^{12 \alpha n_{m+1}}-2 \cdot 2^{12 \alpha n_{m}}\left[\mid \geq 2^{2 \alpha n_{m+1}} / 4\right.\right.$. By Definition (3.13) of the stopping times $T_{f}$, this yields $T_{f, k}(\chi)<2^{12 \alpha n_{m+1}}$ for all $k<\left(2^{2 \alpha n_{m+1}} / 4\right) /\left(2 \cdot 2^{2 n_{m+1}}\right)=2^{2(\alpha-1) n_{m+1}} / 8$. The event $E_{\text {no error }, T_{f}}^{m+1}$ holds, and $2^{2(\alpha-1) n_{m+1}} / 8 \geq 2^{\alpha n_{m+1}}$; recall that $\alpha$ and $n_{m+1} \geq n_{0}$ are large (see Section 2.1). Hence we obtain $\left|S\left(T_{f, k}(\chi)\right)\right| \leq 2^{n_{m+1}}$ for all $k \in\left[0,2^{\alpha n_{m+1}}\right.$ [; recall (7.1). Using Definition (3.13) again, we see that $T_{f, j}(\chi)+2 \cdot 2^{2 n_{m+1}} \leq T_{f, k}(\chi)$ is automatically fulfilled for $j<k$ whenever $T_{f, k}(\chi)<2^{12 \alpha n_{m+1}}$, which is the case at least for $k \in\left[0,2^{\alpha n_{m+1}}\right.$ [. Summarizing, we have proven that the event $E_{\mathrm{stop}, T_{f}}^{m+1}$ holds; recall its definition (3.14).

Lemma 7.6P $P\left[\left(E_{\text {when back recog, } f}^{m+1}\right)^{\mathrm{c}} \cap\left\{\xi \in \Xi_{\text {reconst }, f}^{m}\right\}\right] \leq 0.9^{2^{2 \alpha n} m+1}$.

Proof. We define Bernoulli random variables $Y_{k}, k \geq 0$, by $Y_{k}:=1$ if $v\left(k 2^{\alpha n_{m+1}}\right) \in \mathbb{T}_{f}^{\prime}(\xi, \chi)$, and $Y_{k}:=0$ else. Note that $v\left((k+1) 2^{\alpha n_{m+1}}\right)-v\left(k 2^{\alpha n_{m+1}}\right) \geq 2^{\alpha n_{m+1}}>2 \cdot 2^{12 \alpha n_{m}}$. Also note that

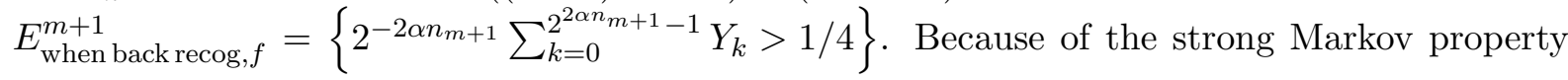


of the random walk $(S(k))_{k \geq 0}$ we have that conditioned under $\xi$ the variables $\left(Y_{k}\right)_{k \geq 0}$ are i.i.d.; recall that $f(\chi)$ depends at most on $\chi\left\lceil\left[0,2 \cdot 2^{12 \alpha n_{m}}\right.\right.$ [. If furthermore $\xi \in \Xi_{\text {reconst, } f}^{m}$ holds, then $E\left[Y_{k} \mid \xi\right] \geq 1 / 2$. Hence we obtain for these $\xi$, using the exponential Chebyshev inequality for the binomial variable $\sum_{k=0}^{2^{2 \alpha n} m+1}-1 Y_{k}$ :

$$
\begin{aligned}
& P\left[\left(E_{\text {when back recog, } f}^{m+1}\right)^{\mathrm{c}} \mid \xi\right]=P\left[2^{-2 \alpha n_{m+1}} \sum_{k=0}^{2^{2 \alpha n}{ }^{m+1}-1} Y_{k} \leq \frac{1}{4} \mid \xi\right] \\
& \leq E\left[e^{1 / 4-Y_{1}} \mid \xi\right]^{2^{2 \alpha n_{m+1}}} \leq\left(\frac{e^{1 / 4}+e^{-3 / 4}}{2}\right)^{2^{2 \alpha n} m+1} \leq 0.9^{2^{2 \alpha n_{m}}+1}
\end{aligned}
$$

This yields the claim of the lemma:

$$
\begin{aligned}
& P\left[\left(E_{\text {when back recog }, f}^{m+1}\right)^{\mathrm{c}} \cap\left\{\xi \in \Xi_{\text {reconst }, f}^{m}\right\}\right] \\
& \leq P\left[\left(E_{\text {when back recog, } f}^{m+1}\right)^{\mathrm{c}} \mid \xi \in \Xi_{\text {reconst }, f}^{m}\right] \leq 0.9^{2^{2 \alpha n_{m+1}}} .
\end{aligned}
$$

Lemma 7.7P $\left[\left(E_{\text {no error }, T_{f}}^{m+1}\right)^{\mathrm{c}} \cap E_{\text {reconst }, f}^{m}\right] \leq \frac{1}{3} e^{-n_{m+1}}$.

Proof. Let $v_{i}$ denote the $(i+1)$ st time when the random walk $S$ visits a point of $\mathbb{Z} \backslash\left[-2^{n_{m+1}}+\right.$ $\left.2 l 2^{12 \alpha n_{m}}, 2^{n_{m+1}}-2 l 2^{12 \alpha n_{m}}\right]$. We set

$$
E_{\mathrm{wrong}, i}^{m+1}:=\left\{\exists w \in \mathcal{C}^{2 \cdot 2^{n_{m}}}: w \preccurlyeq \xi\left[\left[-9 \cdot 2^{n_{m}}, 9 \cdot 2^{n_{m}}\right] \text { and } w \preccurlyeq f\left(\theta^{v_{i}}(\chi)\right)\right\}\right. \text {. }
$$

If the event $E_{\mathrm{wrong}, i}^{m+1}$ occurs, then our procedure might fail to estimate correctly the location of the random walk: we might be misled to think that at time $v_{i}+2 \cdot 2^{12 \alpha n_{m}}$ we are close to the origin while we are not.

We claim that the following holds:

$$
\left(E_{\text {no error }, T_{f}}^{m+1}\right)^{\mathrm{c}} \cap E_{\text {reconst }, f}^{m} \subseteq \bigcup_{i=0}^{2^{12 \alpha n_{m+1}-1}} E_{\text {wrong }, i}^{m+1} .
$$

Indeed: If $\left(E_{\text {no error, } T_{f}}^{m+1}\right)^{\mathrm{c}}$ holds, then $\left|S\left(T_{f, k}(\chi)\right)\right|>2^{n_{m+1}}$ for some $k$ with $T_{f, k}<2^{12 \alpha n_{m+1}}$ (see (7.1)); thus $\left|S\left(T_{f, k}(\chi)-2 \cdot 2^{12 \alpha n_{m}}\right)\right|>2^{n_{m+1}}-2 l 2^{12 \alpha n_{m}}$, since $S$ cannot travel faster than speed $l$. This means $T_{f, k}(\chi)-2 \cdot 2^{12 \alpha n_{m}}=v_{i}$ for some $i<2^{12 \alpha n_{m+1}}$. Using Definition 3.6 of $T_{f, k}(\chi)$, this implies $v_{i} \in \mathbb{T}_{f}(\chi)$; hence there is $w \in \mathcal{C}^{2 \cdot 2^{n} m}$ such that $w \preccurlyeq f(\chi)$ and $w \preccurlyeq f\left(\theta^{v_{i}}(\chi)\right)$. Assuming that the event $E_{\text {reconst }, f}^{m}$ holds, too, this implies $w \preccurlyeq f(\chi) \preccurlyeq \xi\left\lceil\left[-9 \cdot 2^{n_{m}}, 9 \cdot 2^{n_{m}}\right]\right.$; see (3.15). This yields that $E_{\text {wrong }, i}^{m+1}$ holds; recall (7.12). Summarizing, we have shown that (7.13) holds.

For all $i, f\left(\theta^{v_{i}}(\chi)\right)$ depends only on $\chi\left\lceil\left[v_{i}, v_{i}+2 \cdot 2^{12 \alpha n_{m}}\left[\right.\right.\right.$, and $S$ does not visit $\left[-9 \cdot 2^{n_{m}}, 9 \cdot 2^{n_{m}}\right]$ in this time interval $\left[v_{i}, v_{i}+2 \cdot 2^{12 \alpha_{m}}\left[\right.\right.$, since the distance between $\left[-9 \cdot 2^{n_{m}}, 9 \cdot 2^{n_{m}}\right]$ and $\mathbb{Z} \backslash\left[-2^{n_{m+1}}+2 l 2^{12 \alpha n_{m}}, 2^{n_{m+1}}-2 l 2^{12 \alpha n_{m}}\right]$ is larger than $2 l 2^{12 \alpha_{m}}$, and since the random walk cannot travel faster than $l$ steps per time unit. 
Thus by the strong Markov property and by independence of $S$ and $\xi$, we get: $\chi\left\lceil\left[v_{i}, v_{i}+\right.\right.$ $\left.2^{12 \alpha n_{m}}\right]$ is independent of $\xi\left\lceil\left[-9 \cdot 2^{n_{m}}, 9 \cdot 2^{n_{m}}\right]\right.$; therefore $f\left(\theta^{v_{i}}(\chi)\right)$ is independent of $\xi\left\lceil\left[-9 \cdot 2^{n_{m}}, 9\right.\right.$. $\left.2^{n_{m}}\right]$, too.

The probability that a random word of length $2 \cdot 2^{n_{m}}$ which has i.i.d. letters with uniform distribution in $\mathcal{C}$ is equal to a word which is independent of it is equal to $|\mathcal{C}|^{-2 \cdot 2^{n_{m}}}$. There are at most $37 \cdot 2^{n_{m}}$ words of a fixed length in $\xi\left\lceil\left[-9 \cdot 2^{n_{m}}, 9 \cdot 2^{n_{m}}\right]\right.$ and also in $f\left(\theta^{v_{k}}(\chi)\right)$, counting all reversed words, too. Thus there are at most $37^{2} 2^{2 n_{m}}$ pairs of such words. It follows that

$$
P\left[E_{\text {wrong }, i}^{m+1}\right] \leq 37^{2} 2^{2 n_{m}}|\mathcal{C}|^{-2 \cdot 2^{n_{m}}} .
$$

Hence we get the claim of the lemma, using (7.13):

$$
\begin{aligned}
P\left[\left(E_{\text {no error }, T_{f}}^{m+1}\right)^{\mathrm{c}} \cap E_{\text {reconst }, f}^{m}\right] & \leq \sum_{i=0}^{2^{12 \alpha n_{m+1}-1}} P\left[E_{\text {wrong }, i}^{m+1}\right] \\
& \leq 2^{12 \alpha n_{m+1}} \cdot 37^{2} 2^{2 n_{m}}|\mathcal{C}|^{-2 \cdot 2^{n_{m}}} \leq \frac{1}{3} e^{-n_{m+1}} .
\end{aligned}
$$

For the last inequality, recall that $n_{m} \geq n_{0}$ is large enough, and note that $|\mathcal{C}|^{-2 \cdot 2^{n_{m}}}$ is the leading term of the last but one expression; also recall that $n_{m+1}=2^{\left\lfloor\sqrt{n_{m}}\right\rfloor}$ is of a much smaller order than $2^{n_{m}}$.

Proof of Theorem 3.9. By Lemmas 7.4 and 7.5, we know $E_{\text {no error, } T_{f}}^{m+1} \cap E_{\text {enough back }}^{m+1} \cap$ $E_{\text {when back recog, } f}^{m+1} \cap E_{\text {reconst }, f}^{m} \subseteq E_{\text {stop }, T_{f}}^{m+1}$. Using some Boolean algebra, this implies

$$
\begin{aligned}
& \left(E_{\text {stop }, T_{f}}^{m+1}\right)^{\mathrm{c}} \cap E_{\text {reconst }, f}^{m} \cap\left\{\xi \in \Xi_{\text {reconst }, f}^{m}\right\} \\
& \subseteq\left(E_{\text {enough back }}^{m+1}\right)^{\mathrm{c}} \cup\left(\left(E_{\text {no error }, T_{f}}^{m+1}\right)^{\mathrm{c}} \cap E_{\text {reconst }, f}^{m}\right) \cup\left(\left(E_{\text {when back recog, }, f}^{m+1} \cap\right)^{\mathrm{c}} \cap\left\{\xi \in \Xi_{\text {reconst }, f}^{m}\right\}\right) .
\end{aligned}
$$

Consequently, using Definition (7.3) of $\Xi_{\text {reconst, } f}^{m}$ and Lemmas 7.2, 7.6, and 7.7:

$$
\begin{aligned}
& P\left[\left(E_{\text {stop }, T_{f}}^{m+1}\right)^{\mathrm{c}} \cap E_{\text {reconst }, f}^{m} \cap\left\{P\left[E_{\text {reconst }, f}^{m} \mid \xi\right] \geq \frac{1}{2}\right\}\right] \\
& \leq P\left[\left(E_{\text {enough back }}^{m+1}\right)^{\mathrm{c}}\right]+P\left[\left(E_{\text {no error }, T_{f}}^{m+1} \cap E_{\text {reconst }, f}^{m}\right]\right. \\
& \quad+P\left[\left(E_{\text {when back recog, } f}^{m+1}\right)^{\mathrm{c}} \cap\left\{\xi \in \Xi_{\text {reconst }, f}^{m}\right\}\right] \\
& \leq c_{35} 2^{-\alpha n_{m+1}}+\frac{1}{3} e^{-n_{m+1}}+0.9^{2^{2 \alpha n_{m+1}}} \leq e^{-n_{m+1}}
\end{aligned}
$$

recall that $\alpha$ and $n_{m+1} \geq n_{0}$ are large (see Section 2.1). This proves Theorem 3.9.

\section{Getting started: The first stopping times}

In this section, we prove Theorem 3.8 and the related Lemmas 5.2 and 5.3.

\subsection{Properties of the modified measure $\tilde{P}$}

As a preparation of the construction of the first stopping times, we prove some properties of the modified measure $\tilde{P}$. This measure and the closely related measure $P_{\mathrm{B}}$ were introduced in 
Definition 3.2, using an event BigBlock and events $E_{\mathrm{B}}(k)$. Recall that the event $E_{\mathrm{B}}(k)$ holds if there is an initial piece of $k$ 1's in the observation $\chi$, while BigBlock holds if there is a sufficiently large interval of 1 's in the scenery $\xi$ sufficiently close to the origin.

Almost all the proofs using the measure $\tilde{P}$ will not explicitly use its definition (3.5), but only the following properties of $\tilde{P}$ (and $\left.n_{0}\right)$ :

Lemma 8.1 The probability measure $\tilde{P}$ fulfills:

1. $\xi$ and $S$ are independent with respect to $\tilde{P}$;

2. The common distributions of $\left(S, \xi\left\lceil\left(\mathbb{Z} \backslash J_{1}\right)\right)\right.$ with respect to $\tilde{P}$ and with respect to $P$ coincide.

3. With respect to $\tilde{P}$, the restriction $\xi\left\lceil J_{1}\right.$ is independent of $\xi\left\lceil\left(\mathbb{Z} \backslash J_{1}\right)\right.$.

4. $\tilde{P}[$ BigBlock $]=1$.

Proof of Lemma 8.1. We abbreviate $k:=n_{0}^{20}$.

1. We observe first that $\xi \circ \Theta^{k}$ and the event $E_{\mathrm{B}}(k)$ are both measurable with respect to the $\sigma$-field $\sigma\left(\xi,(S(j))_{j \leq k}\right)$, and $S \circ \Theta^{k}$ is measurable with respect to $\sigma\left((S(j)-S(k))_{j>k}\right)$. Since $\sigma\left(\xi,(S(j))_{j \leq k}\right)$ and $\sigma\left((S(j)-S(k))_{j>k}\right)$ are independent with respect to $P$, this implies that $\xi \circ \Theta^{k}$ and $S \circ \Theta^{k}$ are independent with respect to $P\left[\cdot \mid E_{\mathrm{B}}(k)\right]$. Hence $\xi$ and $S$ are independent with respect to the image measure $P_{\mathrm{B}}=\left(P\left[\cdot \mid E_{\mathrm{B}}(k)\right]\right) \circ\left(\Theta^{k}\right)^{-1}$. Since BigBlock $\in \sigma(\xi)$, this implies part 1 .

2. By the independence proven in 1., it suffices to show the two claims $\mathcal{L}_{\tilde{P}}(S)=\mathcal{L}_{P}(S)$ and $\mathcal{L}_{\tilde{P}}\left(\xi\left\lceil\left(\mathbb{Z} \backslash J_{1}\right)\right)=\mathcal{L}_{P}\left(\xi\left\lceil\left(\mathbb{Z} \backslash J_{1}\right)\right):\right.\right.$

- With respect to $P, S \circ \Theta^{k}$ and $S$ both have i.i.d. $\mu$-distributed increments and the starting point 0 ; thus their distributions coincide. By the above argument, $S \circ \Theta^{k}$ and $E_{\mathrm{B}}(k)$ are independent with respect to $P$. Hence the laws of $S \circ \Theta^{k}$ with respect to $P$ and with respect to $P\left[\cdot \mid E_{\mathrm{B}}(k)\right]$ coincide with the law $\mathcal{L}_{P}(S)$ of $S$ with respect to $P$. Hence $\mathcal{L}_{P}(S)=\mathcal{L}_{P\left[\cdot \mid E_{\mathrm{B}}(k)\right]}\left(S \circ \Theta^{k}\right)=\mathcal{L}_{P_{B}}(S)$. Since $\xi$ and $S$ are independent with respect to $P_{B}$, and since BigBlock $\in \sigma(\xi)$, we obtain the first claim $\mathcal{L}_{\tilde{P}}(S)=\mathcal{L}_{P}(S)$.

- We condition on fixed values of $\xi\lceil[-l k, l k]$ and $S\lceil[0, k]$ :

We know that $\xi \circ \Theta^{k}$ is a translation of $\xi$ by $S(k)$ steps, which is not more than $k l$; this translation maps $[-l k, l k]$ to a subset of $J_{1}$. Thus $\left(\xi \circ \Theta^{k}\right)\left\lceil\left(\mathbb{Z} \backslash J_{1}\right)\right.$ is obtained by translating a $(S(k)$-dependent) subpiece of $\xi\lceil(\mathbb{Z} \backslash[-l k, l k])$. Thus by our i.i.d. and independence assumptions for $\xi$ and $S$ we get: $\left(\xi \circ \Theta^{k}\right)\left\lceil\left(\mathbb{Z} \backslash J_{1}\right)\right.$ has the distribution $\mathcal{L}_{P}\left(\xi\left\lceil\left(\mathbb{Z} \backslash J_{1}\right)\right)=\nu^{\mathbb{Z} \backslash J_{1}}\right.$ with respect to $P\left[\cdot \mid \xi\left\lceil[-l k, l k], S\lceil[0, k]]\right.\right.$. Furthermore, ( ${ }^{\circ} \circ$ $\left.\Theta^{k}\right)\left\lceil\left(\mathbb{Z} \backslash J_{1}\right)\right.$ and $\left(\xi \circ \Theta^{k}\right)\left\lceil J_{1}\right.$ are independent with respect to $P[\cdot \mid \xi\lceil[-l k, l k], S\lceil[0, k]]$. Since $E_{\mathrm{B}}(k)$ depends only on $\xi\lceil[-l k, l k]$ and $S\lceil[0, k]$, this implies

$$
\mathcal{L}_{P_{\mathrm{B}}}\left(\xi\left\lceil\left(\mathbb{Z} \backslash J_{1}\right)\right)=\mathcal{L}_{P\left[\cdot \mid E_{\mathrm{B}}(k)\right]}\left(\left(\xi \circ \Theta^{k}\right)\left\lceil\left(\mathbb{Z} \backslash J_{1}\right)\right)=\nu^{\mathbb{Z} \backslash J_{1}},\right.\right.
$$

and $\xi\left\lceil\left(\mathbb{Z} \backslash J_{1}\right)\right.$ is independent of $\xi\left\lceil J_{1}\right.$ with respect to $P_{\mathrm{B}}$. Since the event BigBlock depends only on $\xi\left\lceil J_{1}\right.$, this independence implies

$$
\mathcal{L}_{\tilde{P}}\left(\xi\left\lceil\left(\mathbb{Z} \backslash J_{1}\right)\right)=\mathcal{L}_{P_{\mathrm{B}}}\left(\xi\left\lceil\left(\mathbb{Z} \backslash J_{1}\right)\right)=\nu^{\mathbb{Z} \backslash J_{1}}=\mathcal{L}_{P}\left(\xi\left\lceil\left(\mathbb{Z} \backslash J_{1}\right)\right) ;\right.\right.\right.
$$

recall our choice of $\tilde{P}$. This proves our second claim. 
3. We have just seen: BigBlock $\in \sigma\left(\xi\left\lceil J_{1}\right)\right.$, and the random pieces $\xi\left\lceil\left(\mathbb{Z} \backslash J_{1}\right)\right.$ and $\xi\left\lceil J_{1}\right.$ are mutually independent with respect to $P_{\mathrm{B}}$. These two facts imply part 3 .

4. This is an immediate consequence of the definition $\tilde{P}=P_{\mathrm{B}}[\cdot \mid$ BigBlock $]$.

Definition 8.2 For $k, \kappa \in \mathbb{N}$, let $\Xi_{\text {Block }}(k, \kappa)$ be the event of sceneries

$$
\Xi_{\text {Block }}(k, \kappa):=\left\{\xi \in \mathcal{C}^{\mathbb{Z}} \mid \begin{array}{l}
\text { There is an integer interval } J_{0} \subseteq[-l k, l k] \text { with }\left|J_{0}\right| \geq \kappa \text { such } \\
\text { that } \xi\left\lceil J_{0}=(1)_{j \in J_{0}} \text { is a constant piece of scenery with value } 1 .\right.
\end{array}\right\} .
$$

We are mostly interested in the case $k=n_{0}^{20}, \kappa=n_{0}^{4}$.

Lemma 8.3 If $\kappa \in 2 \mathbb{N}$ is large enough, $k \geq \kappa^{2}, k \in \mathbb{N}$, and if $\xi$ is a scenery with $\xi \notin$ $\Xi_{\text {Block }}(k, \kappa)$, then $P\left[E_{\mathrm{B}}(k) \mid \xi\right] \leq e^{-c_{7} k / \kappa^{2}}$ with some constant $c_{7}>0$. As a consequence, $P\left[E_{\mathrm{B}}(k) \mid \xi \notin \Xi_{\mathrm{Block}}(k, \kappa)\right] \leq e^{-c_{7} k / \kappa^{2}}$.

Proof. Let $\xi \in \mathcal{C}^{\mathbb{Z}} \backslash \Xi_{\text {Block }}(k, \kappa)$. The idea of the proof is to split the time interval $[0, k]$ into pieces of size $\kappa^{2}$. Let us examine at first one of these time intervals of size $\kappa^{2}$ : A typical length scale for the distance that the random walks travels in this time interval is $\kappa$; in particular the probability that it travels farther than distance $\kappa$ is bounded away from 0 , at least if $\kappa$ is large enough. If the random walk travels that far, it gets close by a point not colored with "1", assuming that $\xi \notin \Xi_{\mathrm{Block}}(k, \kappa)$. (Note that the random walk does not leave the interval $[-l k, l k]$ up to the time horizon $k$.) But once the random walk is close enough to a point not colored with "1", the probability to hit this point a few steps later is bounded away from 0 , too. Thus in every $\kappa^{2}$-sized interval the random walk has a probability bounded away from 0 to see not only the color 1 . There are roughly $k / \kappa^{2}$ such intervals in $[0, k]$; thus the probability to see only 1 's up to the time horizon $k$ is exponentially small in $k / \kappa^{2}$.

Formally, we proceed as follows: We define stopping times $\left(\tau_{j}\right)_{j=0, \ldots,\left\lfloor\kappa^{-2} k\right\rfloor-1}$ :

$$
\tau_{j}:=\inf \left\{t \in\left[j \kappa^{2},(j+1 / 2) \kappa^{2}\right] \mid \xi\left\lceil\left[S(t), S(t)+l_{\rightarrow}\right] \text { is not constant } 1\right\} .\right.
$$

In other words, $\tau_{j}$ is the smallest time in the interval $\left[j \kappa^{2},(j+1 / 2) \kappa^{2}\right]$ when there is a point sufficiently close to the right of the location of the random walker which is not colored with " 1 ". If no such time exists, $\tau_{j}=\infty$. We claim: For some constant $c_{8}>0$ holds

$$
P\left[\tau_{j}<\infty \mid \xi, S\left\lceil\left[0, j \kappa^{2}\right]\right] \geq c_{8} .\right.
$$

This means: Uniformly in $\xi \in \mathcal{C}^{\mathbb{Z}} \backslash \Xi_{\text {Block }}(k, \kappa)$ and in the history of the random walker up to time $j \kappa^{2}$, the chance that the random walk will get sufficiently close to a point not colored by "1" during the next $\kappa^{2} / 2$ steps is bounded from below by a positive constant.

To prove (8.5), we observe by the Markov property, $\Delta_{j}:=S\left((j+1 / 2) \kappa^{2}\right)-S\left(j \kappa^{2}\right)$ has the distribution $\mu^{* \kappa^{2} / 2}$ with respect to the conditional law $P_{j}^{\prime}:=P\left[\cdot \mid \xi, S\left\lceil\left[0, j \kappa^{2}\right]\right]\right.$; recall that $\kappa$ is even. Since $\mu^{* \kappa^{2} / 2}$ has the standard deviation $c_{9} \kappa$ for some constant $c_{9}$, the Central Limit Theorem implies

$$
P\left[\Delta_{j} \geq \kappa\right] \geq c_{10}
$$


for large enough $\kappa$. Here $c_{10}$ denotes a fixed positive constant less than $P\left[X \geq c_{9}^{-1}\right]$, and $X$ is a standard normal random variable. Observe that whenever $\xi \in \mathcal{C}^{\mathbb{Z}} \backslash \Xi_{\text {Block }}(k, \kappa)$ and $\Delta_{j} \geq \kappa$ hold (i.e. in the interval of interest the random walk $S$ moves at least the distance $\kappa$ to the right), then $\tau_{j}$ is finite (i.e. the random walk passes close to a point which is not colored with 1 ). This is true since $\xi \in \mathcal{C}^{\mathbb{Z}} \backslash \Xi_{\text {Block }}(k, \kappa)$ implies that $\xi\left\lceil\left[S\left((j+1 / 2) \kappa^{2}\right), S\left((j+1 / 2) \kappa^{2}\right)+\kappa\right]\right.$ cannot be a constant piece 1 , and since the random walk does not perform jumps to the right larger than $l_{\rightarrow}$. Since the jump distribution $\mu$ is not supported on a strict sublattice $\gamma \mathbb{Z}$ of $\mathbb{Z}$, there is a fixed $L \in \mathbb{N}$ such that $\left[0, l_{\rightarrow}\right] \subseteq \bigcup_{\ell=0}^{L} \operatorname{supp}\left(\mu^{* \ell}\right)$. If the event $\left\{\tau_{j}<\infty\right\}$ holds, then $\xi$ is not constant 1 on the interval $\left[S\left(\tau_{j}\right), S\left(\tau_{j}\right)+l_{\rightarrow}\right]$. Let $A_{j}$ denote the event that $\chi\left\lceil\left[j \kappa^{2},(j+1) \kappa^{2}\right]\right.$ is constant 1. Then we have for some constant $1>c_{11}>0$ and $\kappa^{2} / 2 \geq L$ :

$$
\begin{aligned}
P_{j}^{\prime}\left[A_{j}^{\mathrm{c}}\right] & \geq P_{j}^{\prime}\left[\tau_{j}<\infty\right] P_{j}^{\prime}\left[\exists \ell \in[0, L]: \chi\left(\tau_{j}+\ell\right) \neq 1 \mid \tau_{j}<\infty\right] \\
& \geq c_{8} P_{j}^{\prime}\left[\exists \ell \in[0, L]: \chi\left(\tau_{j}+\ell\right) \neq 1 \mid \tau_{j}<\infty\right] \geq c_{11} .
\end{aligned}
$$

Hence we obtain by the Markov property:

$$
P\left[E_{\mathrm{B}}(k) \mid \xi\right] \leq P\left[\bigcap_{j=0}^{\left\lfloor\kappa^{-2} k\right\rfloor-1} A_{j} \mid \xi\right]=E\left[\prod_{j=0}^{\left\lfloor\kappa^{-2} k\right\rfloor-1} P_{j}^{\prime}\left[A_{j}\right] \mid \xi\right] \leq\left(1-c_{11}\right)^{\left\lfloor\kappa^{-2} k\right\rfloor} .
$$

This proves Lemma 8.3

Proof of Lemma 5.2. Let $\sigma^{2}:=\operatorname{Var}[S(1)]$ be the variance of the single step distribution $\mu$. Consider the integer interval $I:=\left[-2 \sigma n_{0}^{10}, 2 \sigma n_{0}^{10}\right] \cap \mathbb{Z}$; then

$$
P\left[\xi\left\lceil I=(1)_{j \in I}\right]=|\mathcal{C}|^{-|I|} \geq|\mathcal{C}|^{-4 \sigma n_{0}^{10}-1} .\right.
$$

The first submartingale inequality states $P\left[\max _{0 \leq j \leq t} X_{j}>\lambda\right] \leq E\left[X_{t}\right] / \lambda$, for $\lambda>0$ and nonnegative submartingales $X_{t}$. Recall that $S^{2}$ is a submartingale, since $x \mapsto x^{2}$ is convex. Applying the submartingale inequality yields:

$$
P\left[\exists j \in\left[0, n_{0}^{20}\right]: S(j) \notin I\right]=P\left[\max _{0 \leq j \leq n_{0}^{20}} S(j)^{2}>4 \sigma^{2} n_{0}^{20}\right] \leq\left(4 \sigma^{2} n_{0}^{20}\right)^{-1} E\left[S\left(n_{0}^{20}\right)^{2}\right]=\frac{1}{4} .
$$

If $S(j) \in I$ is valid for all $j \in\left[0, n_{0}^{20}\right]$ and if $\xi\left\lceil I=(1)_{j \in I}\right.$ holds, then $\chi\left\lceil\left[0, n_{0}^{20}\right]=(1)_{j \in\left[0, n_{0}^{20}\right]}\right.$. Thus (8.9) and (8.10) and the independence of $S$ and $\xi$ imply

$$
P\left[E_{\mathrm{B}}\left(n_{0}^{20}\right)\right] \geq \frac{3}{4}|\mathcal{C}|^{-4 \sigma n_{0}^{10}-1} .
$$

Hence we get for some constant $c_{3}>0$, using Lemma 8.3 and the abbreviations $\Xi_{\text {Block }}^{\mathrm{c}}=$ $\mathcal{C}^{\mathbb{Z}} \backslash \Xi_{\text {Block }}\left(n_{0}^{20}, n_{0}^{4}\right)$ and $E_{\mathrm{B}}=E_{\mathrm{B}}\left(n_{0}^{20}\right)$ :

$$
P\left[\xi \in \Xi_{\text {Block }}^{\mathrm{c}} \mid E_{\mathrm{B}}\right] \leq \frac{P\left[E_{\mathrm{B}} \mid \xi \in \Xi_{\mathrm{Block}}^{\mathrm{c}}\right]}{P\left[E_{\mathrm{B}}\right]} \leq \frac{4}{3}|\mathcal{C}|^{4 \sigma n_{0}^{10}+1} e^{-c_{7} n_{0}^{12}} \leq e^{-c_{3} n_{0}^{12}} .
$$

The shift operation $\Theta^{n_{0}^{20}}$ applied to $(\xi, S)$ cannot shift the scenery $\xi$ by more than $\ln _{0}^{20}$ steps, and every shift of the interval $\left[-\ln _{0}^{20}, \ln _{0}^{20}\right]$ by not more than $l n_{0}^{20}$ steps is contained in $J_{1}$. Thus the shifted event $\Theta^{-n_{0}^{20}}$ BigBlock occurs whenever the event $\xi \in \Xi_{\text {Block }}$ holds; thus (8.12) implies $P\left[\Theta^{-n_{0}^{20}} \operatorname{BigBlock}^{\mathrm{c}} \mid E_{\mathrm{B}}\right] \leq e^{-c_{3} n_{0}^{12}}$, which is equivalent to the claim of Lemma 5.2. 
Proof of Lemma 5.3. Define $\Omega^{\prime}:=\mathcal{C}^{\mathbb{Z} \backslash J_{1}} \times \Omega_{2}$ and write $\Omega=\mathcal{C}^{\mathbb{Z}} \times \Omega_{2}=\mathcal{C}^{J_{1}} \times \mathcal{C}^{\mathbb{Z} \backslash J_{1}} \times \Omega_{2}=$ $\mathcal{C}^{J_{1}} \times \Omega^{\prime}$. Then by definition of the measure $P$ and by Lemma 8.1 we have $P=\nu^{J_{1}} \otimes P_{\Omega^{\prime}}$ and $\tilde{P}=\tilde{P}_{J_{1}} \otimes P_{\Omega^{\prime}}$ where $P_{\Omega^{\prime}}$ and $\tilde{P}_{J_{1}}$, respectively, are the marginal distributions of $\tilde{P}$ on $\Omega^{\prime}$ and $\mathcal{C}^{J_{1}}$, respectively. Thus we have for all measurable cylinder-sets of the form $E=\left\{e_{1}\right\} \times E_{2} \subset \Omega$, where $e_{1} \in \mathcal{C}^{J_{1}}$ and $E_{2} \subseteq \Omega^{\prime}$ :

$$
\tilde{P}[E]=\tilde{P}_{J_{1}}\left[\left\{e_{1}\right\}\right] P_{\Omega^{\prime}}\left[E_{2}\right] \leq|\mathcal{C}|^{4 l n_{0}^{20}+1} \nu^{J_{1}}\left[\left\{e_{1}\right\}\right] P_{\Omega^{\prime}}\left[E_{2}\right]=|\mathcal{C}|^{4 l n_{0}^{20}+1} P[E]
$$

where the inequality follows because $\nu$ is the uniform distribution on $\mathcal{C},\left|J_{1}\right|=4 \ln _{0}^{20}+1$, and $\tilde{P}_{J_{1}}$ is bounded from above by one. Since $\mathcal{C}^{J_{1}}$ is finite, every measurable subset of $\Omega$ can be written as a finite disjoint union of sets of the above form $\left\{e_{1}\right\} \times E_{2}$ with $e_{1} \in \mathcal{C}^{J_{1}}$ and $E_{2} \subseteq \Omega^{\prime}$. This proves the result.

\subsection{The stopping times $T^{0}$}

We start with the definition of a sequence $T^{0}=\left(T_{k}^{0}\right)_{k \geq 0}$ of $\mathcal{G}$-adapted stopping times with values in $\left[0,2^{12 \alpha n_{0}}\right]$. Roughly speaking, these times search for long blocks of 1 's in the observation $\chi$. Here is intuitive idea behind this construction: Since we conditioned on a large block of 1's to occur in the true scenery $\xi$ close to the origin, observing a long block of 1's at a later time indicates with high probability that the random walk has returned close to the origin. This is true only up to a certain time horizon, since long blocks of 1's in the true scenery will occur far from the origin, as well. In turns out that the appropriate time horizon is of length $2^{12 \alpha n_{0}}$. Hence we define our first stopping times and the corresponding events as follows.

Definition 8.4 Let the random set $\mathbb{T}^{0}(\chi)$ be defined as follows:

$$
\mathbb{T}^{0}(\chi):=\left\{t \in \left[0,2^{12 \alpha n_{0}}-n_{0}^{7}\left[\mid \chi\left\lceil\left[t, t+n_{0}^{7}\right] \text { is constant } 1\right\} .\right.\right.\right.
$$

We arrange the elements of $\mathbb{T}^{0}(\chi)$ in increasing order: $t_{0}(0)<\ldots<t_{0}\left(\left|\mathbb{T}^{0}(\chi)\right|-1\right)$. We set

$$
T_{k}^{0}(\chi):= \begin{cases}t_{0}\left(2 \cdot 2^{2 n_{0}} k\right)+n_{0}^{7} & \text { if } 2 \cdot 2^{2 n_{0}} k<\left|\mathbb{T}^{0}(\chi)\right|, \\ 2^{12 \alpha n_{0}} & \text { otherwise. }\end{cases}
$$

Finally, we define the following variant of the event $E_{\text {stop }, T^{0}}^{0}$ defined in (3.14):

$$
\tilde{E}_{\text {stop }, T^{0}}^{0}=\bigcap_{k=0}^{2^{\alpha n_{0}}}\left\{T_{k}^{0}(\chi)<2^{12 \alpha n_{0}},\left|S\left(T_{k}^{0}(\chi)\right)\right| \leq 2^{n_{0} / 2}, T_{j}^{0}(\chi)+2 \cdot 2^{2 n_{0}} \leq T_{k}^{0}(\chi) \text { for } j<k\right\} \text {. }
$$

Comparing this Definition (8.16) with the Definition (3.14) of the event $E_{\text {stop }, T^{0}}^{0}$,

$$
E_{\text {stop }, T^{0}}^{0}=\bigcap_{k=0}^{2^{\alpha n_{0}}}\left\{T_{k}^{0}(\chi)<2^{12 \alpha n_{0}},\left|S\left(T_{k}^{0}(\chi)\right)\right| \leq 2^{n_{0}}, T_{j}^{0}(\chi)+2 \cdot 2^{2 n_{0}} \leq T_{k}^{0}(\chi) \text { for } j<k\right\},
$$

one sees the only difference between the two events: The new event $\tilde{E}_{\mathrm{stop}, T^{0}}^{0}$ requires the random walk $S$ to be much closer to the origin at the stopping times $T^{0}$ than the old event $E_{\text {stop }, T^{0}}^{0}$ does, using the new length scale $2^{n_{0} / 2}$ rather than $2^{n_{0}}$. In particular, we have

$$
\tilde{E}_{\text {stop }, T^{0}}^{0} \subseteq E_{\text {stop }, T^{0}}^{0} .
$$


Theorem 8.5 For some positive constants $c_{36}$ and $c_{37}$ it holds

$$
\tilde{P}\left[E_{\text {stop }, T^{0}}^{0}\right] \geq \tilde{P}\left[\tilde{E}_{\text {stop }, T^{0}}^{0}\right] \geq 1-c_{36} e^{-c_{37} n_{0}} .
$$

We prepare the proof of Theorem 8.5 by some definitions and lemmas. We use again the abbreviation $J_{1}=\left[-2 \ln _{0}^{20}, 2 \ln _{0}^{20}\right]$. Analogously to Definition (7.2), we define events $\tilde{E}_{\text {enough back }, I}^{0}$ :

Definition 8.6 Let $I \subseteq J_{1}$ be an integer interval. We define

$\tilde{E}_{\text {enough back }, I}^{0}:=\left\{\mathrm{Up}\right.$ to time $2^{12 \alpha n_{0}} / 4, S$ visits the interval $I$ at least $2^{3 \alpha n_{0}}$ times $\}$.

Next we will see that these "frequent returns" to the interval $I$ in the first time steps have large probability.

Lemma 8.7 For some constants $c_{38}>0$ and $c_{39}>0$, the following holds: If $I \subseteq J_{1},|I| \geq l$, is an integer interval, then

$$
\tilde{P}\left[\tilde{E}_{\text {enough back }, I}^{0}\right] \geq 1-c_{38} e^{-c_{39} n_{0}} .
$$

Proof. Let $T_{I}:=\inf \{t \mid S(t) \in I\}$ be the entrance time of $S$ into $I$. We show first: For some positive constants $c_{40}$ and $c_{41}$ (depending at most on the distribution $\mu$ of $S(1)$ ) we have:

$$
\tilde{P}\left[T_{I} \geq 2^{12 \alpha n_{0}} / 8\right] \leq c_{40} e^{-c_{41} n_{0}} .
$$

If $0 \in I$, this is trivial, since $S$ starts in 0 . Otherwise $I$ contains only positive numbers, or it contains only negative numbers; without loss of generality we assume the first possibility. Let $\left.z=\min I \in] 0,2 \ln _{0}^{20}\right]$. Consider the interval $\left.J:=\right]-2^{n_{0}}, z[\subseteq]-2^{n_{0}}, 2^{n_{0}}$ [, and consider the exit time $H:=\inf \{t \mid S(t) \notin J\}$ of $J$. Note that $H$ is a.s. finite.

On the one hand, we know

$$
\tilde{P}[H \geq t] \leq c_{42} e^{-c_{43} 2^{-2 n_{0}} t}
$$

for some constants $c_{42}, c_{43}>0$ depending at most on the variance of $S(1)$, since in every time interval of size $2^{2 n_{0}}$ the random walk has a positive probability to exit $J$, bounded away from 0 . In particular, for $t=2^{12 \alpha n_{0}} / 8$ the probability in (8.23) is superexponentially small in $n_{0}$, if $\alpha$ is large enough (see Subsection 2.1).

On the other hand, since $S$ is a martingale and since $S$ has jump sizes bounded by $l$, we get $\tilde{P}[S(H)>0] \geq 1-(z+l) 2^{-n_{0}}$. Furthermore, using again that $S$ has jump sizes bounded by $l$, we know the following: If $S(H)>0$, then $S(H) \in I$ and $T_{I}=H$, since $z$ is the leftmost point in $I$ and $|I| \geq l$; the random walk cannot cross $I$ without touching it.

Altogether, we have the following upper bound for the left hand side in (8.22):

$$
\tilde{P}\left[H \geq 2^{12 \alpha n_{0}} / 8 \text { or } S(H)<0\right] \leq c_{40} e^{-c_{41} n_{0}} .
$$

for some positive constants $c_{40}$ and $c_{41}$.

Provided the random walk visits a point $x \in I$, the probability to visit this point again at least $2^{3 \alpha n_{0}}$ times in the subsequent $2^{12 \alpha n_{0}} / 8$ time steps is at least $1-c_{28} 2^{-\alpha n_{0}}$. This follows from Lemma 7.2, using the strong Markov property of the random walk; recall that the law of $S$ with respect to $P$ and with respect to $\tilde{P}$ coincide. Combining this with (8.22) yields claim (8.21) of Lemma 8.7. 
We remark: Lemma 8.7 holds not only for deterministic intervals $I$, but also for random ones, provided that $I$ and $S$ are independent. We use this below for the following specific choice of $I$, which depends on the scenery $\xi$, but not on $S$ :

$\tilde{P}$-a.s. there is a (random) integer interval $J_{0} \subseteq J_{1}=\left[-2 \ln _{0}^{20}, 2 \ln _{0}^{20}\right]$ with $\left|J_{0}\right| \geq n_{0}^{4}$ such that $\xi\left\lceil J_{0}\right.$ is constant 1 ; recall Definition 3.2. Just for definiteness we take the rightmost such $J_{0}$. Let

$$
I=I(\xi):=\left\{z \in J_{0} \mid \operatorname{dist}\left(z, \mathbb{Z} \backslash J_{0}\right)>n_{0}^{4} / 4\right\} ;
$$

then $I$ is $\tilde{P}$-a.s. well defined, and it is an integer interval containing $|I| \geq n_{0}^{4} / 2 \geq l$ points.

The following is a modification of Definition 7.3. We define those times $t$, for which the random walk is in the random set $I(\xi)$ and does not travel further than distance $n_{0}^{4} / 4$ in the next $n_{0}^{7}$ steps. Correspondingly, we define a new version of the event $E_{\text {when back recog given in }}$ Definition 7.3, that specifies that sufficiently many of the visits to $I(\xi)$ fulfill this requirement.

Definition 8.8 Let $w(k), k \geq 0$, denote the $(k+1)$ st visit to the (random) set $I(\xi)$ by the random walk $S$. We introduce a random set $\mathbb{T}^{0 \prime}$ and an event $\tilde{E}_{\text {when back recog }}^{0}$ :

$$
\begin{aligned}
& \mathbb{T}^{0 \prime}:=\left\{t \in \mathbb{N} \mid S(t) \in I(\xi) \text { and }|S(j)-S(t)| \leq n_{0}^{4} / 4 \text { for } 0 \leq j-t \leq n_{0}^{7}\right\} \\
& \tilde{E}_{\text {when back recog }}^{0}:=\left\{\text { For more than } 1 / 4 \text { of the points } k \in \left[0,2^{2 \alpha n_{0}}\left[\text { holds } w\left(k 2^{\alpha n_{0}}\right) \in \mathbb{T}^{0 \prime}\right\}\right.\right.
\end{aligned}
$$

Furthermore, we set

$$
\tilde{E}_{\text {no error }, T^{0}}^{0}:=\left\{\forall k \in \mathbb{N}: \text { If } T_{k}^{0}(\chi)<2^{12 \alpha n_{0}} \text {, then }\left|S\left(T_{k}^{0}(\chi)\right)\right| \leq 2^{n_{0} / 2}\right\} .
$$

Note that (8.28) uses again the length scale $2^{n_{0} / 2}$, in contrast to the length scale $2^{n_{m}}$ in Definition (7.1).

We prove the following modification of Lemma 7.6 , that shows that $\tilde{E}_{\text {when back recog }}^{0}$ is a likely event.

Lemma $8.9 \tilde{P}\left[\left(\tilde{E}_{\text {when back recog }}^{0}\right)^{\mathrm{c}}\right] \leq 0.9^{2^{2 \alpha n_{0}}}$.

Proof. We observe as in (8.10) by the submartingale inequality:

$$
P\left[|S(j)| \leq n_{0}^{4} / 4 \text { for } 0 \leq j \leq n_{0}^{7}\right] \geq 1-4^{2} n_{0}^{-8} E\left[S\left(n_{0}^{7}\right)^{2}\right]=1-\frac{16 \operatorname{Var}[S(1)]}{n_{0}} \geq \frac{1}{2},
$$

since $n_{0}$ is large enough; see Subsection 2.1. Let $Y_{k}$ denote the indicator function of the event $\left\{w\left(k 2^{\alpha n_{0}}\right) \in \mathbb{T}^{0 /}\right\}$; the $Y_{k}$ are $\tilde{P}$-a.s. well defined. As a consequence of the strong Markov property, the $Y_{k}, k \in\left[0,2^{2 \alpha n_{0}}\right.$ [, are i.i.d. Bernoulli random variables; note that the stopping times $w\left(k 2^{\alpha n_{0}}\right), k \in \mathbb{N}$, have at least the spacing $2^{\alpha n_{0}}>n_{0}^{7}$. Furthermore $\tilde{P}\left[Y_{k}=1\right] \geq 1 / 2$, since this probability equals the left hand side in (8.29); recall that the laws of $S$ with respect to $P$ and with respect to $\tilde{P}$ coincide. The claim of the Lemma now follows by the same large deviation argument as in (7.10).

Next, we claim the following analogue of Lemma 7.4:

Lemma 8.10 The inclusion $\mathbb{T}^{0} \supseteq \mathbb{T}^{0 \prime} \cap\left[0,2^{12 \alpha n_{0}}-n_{0}^{7}[\right.$ holds $\tilde{P}$-almost surely. 
Proof. Assume that the event BigBlock holds; this occurs $\tilde{P}$-almost surely. Then $I(\xi)$ is well defined. Let $t \in \mathbb{T}^{0 \prime}, t<2^{12 \alpha n_{0}}-n_{0}^{7}$. Then $S(t) \in I(\xi)$, and during the subsequent $n_{0}^{7}$ steps, the random walk $S$ cannot leave the interval $J_{0}$, since it does not travel farther than $n_{0}^{4} / 4$ (recall definition $(8.26)$ ), and since $\mathbb{Z} \backslash J_{0}$ is more distant than this from $I(\xi)$ (recall the definition of $I(\xi))$. Since $\xi\left\lceil J_{0}\right.$ is constant 1 by definition (3.4) of the event BigBlock, this implies that $S\left\lceil\left[t, t+n_{0}^{7}\right]\right.$ is constant 1 ; i.e. $t \in \mathbb{T}^{0}$.

The following Lemma is a slight modification of Lemma 7.5; except little differences, the proofs are also very similar.

Lemma 8.11 Assume that the events

$$
\tilde{E}_{\text {no error }, T^{0}}^{0} \cap \tilde{E}_{\text {enough back }, I(\xi)}^{0} \cap \tilde{E}_{\text {when back recog }}^{0} \quad \text { and } \quad \mathbb{T}^{0}(\chi) \supseteq \mathbb{T}^{0 \prime} \cap\left[0,2^{12 \alpha n_{0}}-n_{0}^{7}[\right.
$$

hold. Then $\tilde{E}_{\mathrm{stop}, T^{0}}^{0}$ holds, too.

Proof. Using $\tilde{E}_{\text {enough back }, I(\xi)}^{0}$, we know

$$
w\left(k 2^{\alpha n_{0}}\right) \in\left[0,2^{12 \alpha n_{0}} / 4\right] \subseteq\left[0,2^{12 \alpha n_{0}}-n_{0}^{7}[\right.
$$

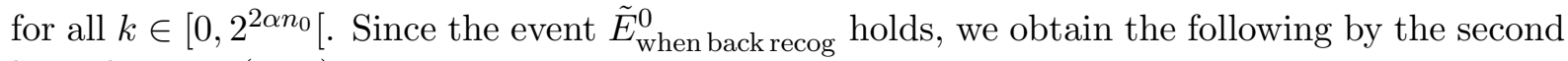
hypothesis in (8.30):

$$
\left|\mathbb{T}^{0}(\chi)\right| \geq \mid \mathbb{T}^{0 \prime} \cap\left[0,2^{12 \alpha n_{0}}-n_{0}^{7}\left[\mid \geq 2^{2 \alpha n_{0}} / 4 .\right.\right.
$$

By Definition (8.15) of the stopping times $T^{0}$, this yields $T_{k}^{0}(\chi)<2^{12 \alpha n_{0}}$ for all $k<\left(2^{2 \alpha n_{0}} / 4\right) /(2$. $\left.2^{2 n_{0}}\right)=2^{2(\alpha-1) n_{0}} / 8$. The event $\tilde{E}_{\text {no error }, T^{0}}^{0}$ holds, and $2^{2(\alpha-1) n_{0}} / 8 \geq 2^{\alpha n_{0}}$; recall that $\alpha$ and $n_{0}$ are large (see Section 2.1). Hence we obtain $\left|S\left(T_{k}^{0}(\chi)\right)\right| \leq 2^{n_{0} / 2}$ for all $k \in\left[0,2^{\alpha n_{0}}[\right.$. Using Definition (8.15) again, we conclude that $T_{j}^{0}(\chi)+2 \cdot 2^{2 n_{0}} \leq T_{k}^{0}(\chi)$ is valid for $j<k$ whenever $T_{k}^{0}(\chi)<2^{12 \alpha n_{0}}$, which is the case at least for $k \in\left[0,2^{\alpha n_{0}}\right.$ [. Summarizing, we have proven that the event $\tilde{E}_{\text {stop }, T^{0}}^{0}$ holds; recall its definition (8.16).

Next we defines those sceneries that do not contain long (i.e. longer than $n_{0}^{2}$ ) blocks of ones around the origin apart from those contained in $J_{1}$.

Definition 8.12 We define the event of sceneries

$$
\Xi_{\text {no blocks }}^{0}:=\left\{\xi \in \mathcal{C}^{\mathbb{Z}} \mid \begin{array}{l}
\text { For every (integer) interval } J \subseteq\left[-2 l 2^{12 \alpha n_{0}}, 2 l 2^{12 \alpha n_{0}}\right] \backslash J_{1} \\
\text { with }|J|=n_{0}^{2} \text { it holds: } \xi\lceil J \text { is not constant } 1 .
\end{array}\right\}
$$

These sceneries turn out to be sufficiently likely.

Lemma 8.13 For some positive constants $c_{44}, c_{45}$ holds $\tilde{P}\left[\xi \in \Xi_{\text {noblocks }}^{0}\right] \geq 1-c_{44} e^{-c_{45} n_{0}}$.

Proof. For every fixed interval $J \subseteq\left[-2 \cdot l 2^{12 \alpha n_{0}}, 2 \cdot l 2^{12 \alpha n_{0}}\right] \backslash J_{1}$ with $|J|=n_{0}^{2}$ we have

$$
\tilde{P}\left[\xi\lceil J \text { is constant } 1]=|\mathcal{C}|^{-n_{0}^{2}},\right.
$$

which is superexponentially small in $n_{0}$. Furthermore, there are less than $4 l 2^{12 \alpha n_{0}}$ such intervals. Thus $\tilde{P}\left[\xi \notin \Xi_{\text {no blocks }}^{0}\right] \leq 4 l 2^{12 \alpha n_{0}}|\mathcal{C}|^{-n_{0}^{2}}$, which is still superexponentially small in $n_{0}$. This implies the lemma. Note that we may choose $c_{44}, c_{45}$ independent of $\alpha$ for $n_{0}$ large enough, even though $4 l 2^{12 \alpha n_{0}}|\mathcal{C}|^{-n_{0}^{2}}$ does depend on $\alpha$ (see Subsection 2.1). 
Lemma 8.14 For some constants $c_{46}, c_{47}>0$ holds

$$
\tilde{P}\left[\left(\tilde{E}_{\text {no error, } T^{0}}^{0}\right)^{\mathrm{c}}\right] \leq c_{46} e^{-c_{47} n_{0}} .
$$

Proof. Let $\mathcal{X}$ be defined by

$$
\mathcal{X}:=\left\{x \in \mathbb{Z} \mid x+\left[-\ln _{0}^{7}, \ln _{0}^{7}\right] \subseteq\left[-2 l 2^{12 \alpha n_{0}}, 2 l 2^{12 \alpha n_{0}}\right] \backslash J_{1}\right\} .
$$

As a consequence of Lemma 8.3 (with the parameters $k=n_{0}^{7}$ and $\kappa=n_{0}^{2}$ ) we know for every $\xi \in \mathcal{C}^{\mathbb{N}}$ such that $\xi\left\lceil\left[-\ln _{0}^{7}, \ln _{0}^{7}\right]\right.$ contains no block of 1 's of length $n_{0}^{2}$ :

$$
P_{\xi}\left[\xi \circ S\left\lceil\left[0, n_{0}^{7}\right] \text { is constant } 1\right] \leq e^{-c_{7} n_{0}^{3}} .\right.
$$

Let $t \in \mathbb{N}$ and let $\xi \in \Xi_{\text {no blocks }}^{0}$. Using the Markov property of the random walk, (8.37) implies the following:

$$
P_{\xi}\left[\xi \circ S\left\lceil\left(t+\left[0, n_{0}^{7}\right]\right) \text { is constant } 1 \mid S(t) \in \mathcal{X}\right] \leq e^{-c_{7} n_{0}^{3}} .\right.
$$

If $t<2^{12 \alpha n_{0}}$ and $|S(t)|>2^{n_{0} / 2}$ holds, then we know $S(t) \in \mathcal{X}$; note that $J_{1}=\left[-2 \ln _{0}^{20}, 2 \ln _{0}^{20}\right]$ has a distance larger than $\ln _{0}^{7}$ from $\mathbb{Z} \backslash\left[-2^{n_{0} / 2}, 2^{n_{0} / 2}\right]$, and recall that $S$ cannot travel faster than with speed $l$, and that $n_{0}$ is large by Subsection 2.1 .

Thus (8.38) implies

$$
\begin{aligned}
& P_{\xi}\left[\left(E_{\text {no error, } T^{0}}^{0}\right)^{\mathrm{c}}\right] \\
& \leq P_{\xi}\left[\text { There is } t<2^{12 \alpha n_{0}} \text { such that }|S(t)|>2^{n_{0} / 2} \text { and } \xi \circ S\left\lceil\left(t+\left[0, n_{0}^{7}\right]\right) \text { is constant } 1\right]\right. \\
& \leq 2^{12 \alpha n_{0}} e^{-c_{7} n_{0}^{3}} \leq e^{-n_{0}}
\end{aligned}
$$

for the last inequality recall that $n_{0}$ was chosen large enough, depending on $\alpha$ (see Subsection 2.1). Combining this with Lemma 8.13 yields for some positive constants $c_{46}, c_{47}$ :

$$
\tilde{P}\left[\left(\tilde{E}_{\text {no error }, T^{0}}^{0}\right)^{\mathrm{c}}\right] \leq \tilde{P}\left[\xi \notin \Xi_{\text {no blocks }}^{0}\right]+\int_{\left\{\xi \in \Xi_{\text {no blocks }}^{0}\right\}} P_{\xi}\left[\left(E_{\text {no error }, T^{0}}^{0}\right)^{\mathrm{c}}\right] d \tilde{P} \leq c_{46} e^{-c_{47} n_{0}} .
$$

Proof of Theorem 8.5. From Lemmas 8.10 and 8.11 we know that

$$
\tilde{P}\left[\left(\tilde{E}_{\text {stop }, T^{0}}^{0}\right)^{\mathrm{c}}\right] \leq \tilde{P}\left[\left(\tilde{E}_{\text {no error }, T^{0}}^{0}\right)^{\mathrm{c}}\right]+\tilde{P}\left[\left(\tilde{E}_{\text {enough back }, I(\xi)}^{0}\right)^{\mathrm{c}}\right]+\tilde{P}\left[\left(\tilde{E}_{\text {when back recog }}^{0}\right)^{\mathrm{c}}\right] .
$$

Hence the claim of Theorem 8.5 is a consequence of Lemmas 8.7, 8.9, and 8.14.

\subsection{The stopping times $T^{1}$}

Unfortunately, the constructed stopping times $T^{0}$ are not good enough as arguments for the first reconstruction Algorithm " $\operatorname{Alg}^{n}$ ": We cannot construct more than roughly $\exp \left(\operatorname{const} n_{0}^{4}\right)$ reliable stopping times based on the way we build the $T^{0}$ stopping times; our actual construction uses only $2^{\alpha n_{0}} \ll \exp \left(\operatorname{const} n_{0}^{4}\right)$ of these stopping times. If we were using too many stopping times of the type $T^{0}$, we could not guarantee that they really stop the random walk with high probability close to the origin. However, the number $\exp \left(\right.$ const $\left.n_{0}^{4}\right)$ is much too small to collect a sufficiently large puzzle for reconstructing at least the modified piece $\xi\left\lceil J_{1}\right.$ in the scenery using our reconstruction algorithm. To illustrate this fact, we remark that we have only roughly an 
upper bound $d \tilde{P} / d P \leq \exp \left(\right.$ const $\left.n_{0}^{20}\right)$; see Lemma 5.3. A modification of the parameters does not solve this problem. The reconstruction algorithm "Alg" needs as input data sufficiently reliable stopping times, which stop the random walk close to the origin; but the stopping times $T^{0}$ are not reliable enough for this purpose. Thus we need an essentially improved series of stopping times $T^{1}$ to get the reconstruction algorithm started.

Our construction of $T^{1}$ is partially parallel to the construction of the partial reconstruction algorithm $\mathrm{Alg}^{n}$, but it is also partially parallel to the construction of the stopping times $T_{f}$ and $T^{0}$ : Roughly speaking, we collect a set of typical signals ("a puzzle") at the very beginning and another one at a candidate time. Instead of matching the pieces together, we just compare the two puzzles: If the puzzles have a sufficiently high overlap, then they were generated with high probability at roughly the same location.

Fortunately, many constructions of the previous sections can be used again, up to small modifications: There are extra complications due to the presence of a modified domain $J_{1}$. We keep the presentation rather close to the previous sections to show the parallelism. Here is the formal definition of the "new" puzzles and of $T^{1}$ :

Definition 8.15 We set, using the abbreviation $\operatorname{Input}_{0}:=\left(T^{0}(\chi), \chi\left\lceil\left[0,2 \cdot 2^{12 \alpha n_{0}}[)\right.\right.\right.$ :

$$
\begin{aligned}
& \operatorname{Puzzle}_{\mathrm{III}}^{n_{0}}(\chi):= \\
& \left\{\left(w_{1}, w_{2}, w_{3}\right) \in \text { Puzzle }_{\mathrm{I}}^{n_{0}}\left(\text { Input }_{0}\right) \mid \exists k \in\left[0,2^{\alpha n_{0}}\left[: w_{1} w_{2} w_{3} \sqsubseteq \chi\left\lceil\left(T_{k}^{0}(\chi)+\left[0,2^{n_{0}} / l\right]\right)\right\},\right.\right.\right. \\
& \text { Puzzle }_{\mathrm{IV}}^{n_{0}}(\chi):=\left\{w_{2} \in \mathcal{C}^{c_{1} n_{0}} \mid \exists w_{1}, w_{3} \in \mathcal{C}^{c_{1} n_{0}}:\left(w_{1}, w_{2}, w_{3}\right) \in \text { Puzzle }_{\mathrm{III}}^{n_{0}}(\chi)\right\}, \\
& \mathbb{T}^{1}(\chi):=\left\{t \in \left[0,2^{12 \alpha n_{1}}-2 \cdot 2^{12 \alpha n_{0}}\left[\begin{array}{l}
\mid P_{\text {uzzle }} \operatorname{IV}_{\mathrm{IV}}(\chi) \cap \text { Puzzle }_{\mathrm{IV}}^{n_{0}}\left(\theta^{t} \chi\right) \mid \geq 2^{n_{0} / 3} \\
\text { and } \mid \text { Puzzle }_{\mathrm{IV}}^{n_{0}}\left(\theta^{t} \chi\right) \mid \leq 50 \cdot 2^{n_{0}}
\end{array}\right\} .\right.\right.
\end{aligned}
$$

Finally we define another sequence $T^{1}=\left(T_{k}^{1}\right)_{k \geq 0}$ of $\mathcal{G}$-adapted stopping times with values in $\left[0,2^{12 \alpha n_{1}}\right]$ : Let $t_{1}(0)<\ldots<t_{1}\left(\left|\mathbb{T}^{1}(\chi)\right|-1\right)$ be the elements of $\mathbb{T}^{1}(\chi)$ arranged in increasing order. For $k \in \mathbb{N}$, we set

$$
T_{k}^{1}(\chi):= \begin{cases}t_{1}\left(2 \cdot 2^{2 n_{1}} k\right)+2 \cdot 2^{12 \alpha n_{0}} & \text { if } 2 \cdot 2^{2 n_{1}} k<\left|\mathbb{T}^{1}(\chi)\right|, \\ 2^{12 \alpha n_{1}} & \text { otherwise }\end{cases}
$$

Note that $T^{0}(\chi)$ only depends on $\chi\left\lceil\left[0,2^{12 \alpha n_{0}}\left[\right.\right.\right.$; thus Puzzle $\mathrm{e}_{\mathrm{IV}}(\chi)$ only depends on $\chi\left\lceil\left[0,2 \cdot 2^{12 \alpha n_{0}}[\right.\right.$, since $2^{n_{0}} / l \leq 2^{12 \alpha n_{0}}$.

The reason why we introduce Puzzle $e_{I I}$ and Puzzle $e_{I V}$, rather than using Puzzle $e_{I}$ and Puzzle again, is explained intuitively before Lemma 8.23, below.

Next we introduce sets to control the position of the words in Puzzle $\operatorname{IV}_{\mathrm{IV}}(\chi)$. The idea behind the following construction is roughly that from words read in $J_{1}$ we obtain information that could be potentially misleading. Moreover, the sets Center $_{I I}$ and Center $_{I I I}$ defined below will serve as potential upper and lower bounds, respectively, for the sets of puzzle words Puzzle $\mathrm{IV}_{\mathrm{IV}}^{n_{0}}(\chi)$. Hence with their help we will control the overlap between the words in Puzzle $e_{\mathrm{IV}}^{n_{0}}(\chi)$ and in Puzzle $\mathrm{IV}_{\mathrm{IV}}^{n_{0}}\left(\theta^{t} \chi\right)$.

Definition 8.16 Using the abbreviation $J_{1}=\left[-2 \ln n_{0}^{20}, 2 \ln n_{0}^{20}\right]$ from Definition 3.2 again, we 
define the following random sets:

CorPaths :=

$\left\{R \in \mathbb{Z}^{\left[0, c_{1} n_{0}[\right.} \mid \begin{array}{l}R \text { is an admissible piece of path, for every admissible piece of path } \\ R^{\prime}:\left[0, c_{1} n_{0}\left[\rightarrow \mathbb{Z} \text { with } R^{\prime}(0)=R(0) \text { and } R^{\prime}\left(c_{1} n_{0}-1\right)=R\left(c_{1} n_{0}-1\right)\right.\right. \\ \text { holds } \xi \circ R^{\prime}=\xi \circ R, \text { and there is such a path } R^{\prime} \text { which takes at } \\ \text { least one value in } J_{1} .\end{array}\right\}$,

Corrupted $:=\left\{\xi \circ R \in \mathcal{C}^{c_{1} n_{0}} \mid R \in\right.$ CorPaths $\}$,

Center $_{I}:=\left\{w \in \mathcal{C}^{c_{1} n_{0}} \mid w\right.$ is a (left or right) ladder word of $\xi\left\lceil\left(\left[-11 \cdot 2^{n_{0}}, 11 \cdot 2^{n_{0}}\right] \backslash J_{1}\right)\right\}$,

Center $_{\text {II }}:=$ Center $_{\mathrm{I}} \cup$ Corrupted,

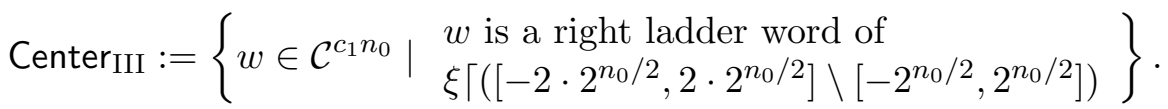

For the following lemma it will be helpful to recall the Definition 6.15 of the event $B_{\text {unique fit }}^{n_{0}}\left(J_{1}\right)$. This events basically says that no two $i$ - resp. $j$ - spaced words $\xi\left\lceil I\right.$, resp. $\xi\left\lceil J\right.$ in $\left[-11 \cdot 2^{n_{0}},-11\right.$. $\left.2^{n_{0}}\right] \backslash J_{1}$ look the same, no matter of they are both read in the same or in different directions.

Lemma 8.17 There exist constants $c_{18}, c_{19}>0$ such that the following holds:

$$
\tilde{P}\left[\left(B_{\text {unique fit }}^{n_{0}}\left(J_{1}\right)\right)^{\mathrm{c}}\right] \leq c_{18} e^{-c_{19} n_{0}} .
$$

Proof. We observe that the event $B_{\text {unique fit }}^{n_{0}}\left(J_{1}\right)$, introduced in Definition 6.15, depends only on the part $\xi\left\lceil\left(\mathbb{Z} \backslash J_{1}\right)\right.$ of the scenery outside the region $J_{1}$. Since the probability measures $P$ and $\tilde{P}$ coincide on the $\sigma$-algebra generated by $\xi\left\lceil\left(\mathbb{Z} \backslash J_{1}\right)\right.$ and $S$ according to part 2 in Lemma 8.1, this implies

$$
\tilde{P}\left[\left(B_{\text {unique fit }}^{n_{0}}\left(J_{1}\right)\right)^{\mathrm{c}}\right]=P\left[\left(B_{\text {unique fit }}^{n_{0}}\left(J_{1}\right)\right)^{\mathrm{c}}\right] .
$$

Thus the claim (8.51) follows from Lemma 6.33.

The next lemma controls the size of our upper and lower bounds Center ${ }_{I I}$ and Center ${ }_{I I I}$, respectively.

Lemma 8.18 $\mid$ Center $_{\mathrm{I}}\left|\leq 46 \cdot 2^{n_{0}},\right|$ Corrupted $\mid \leq n_{0}^{41}$, and thus $\mid$ Center $_{\mathrm{II}} \mid \leq 50 \cdot 2^{n_{0}}$. If the event $B_{\text {unique fit }}^{n_{0}}\left(J_{1}\right)$ holds, then $\mid$ Center $I I I \mid \geq 2^{n_{0} / 3}$.

Proof. The first statement is obvious, since there are at most $23 \cdot 2^{n_{0}}$ choices for the leftmost point of a ladder interval in $\left[-11 \cdot 2^{n_{0}}, 11 \cdot 2^{n_{0}}\right]$, and there is the binary choice "left" or "right".

We show $\mid$ Corrupted $\mid \leq n_{0}^{41}$ next: The number of pairs $\left(R(0), R\left(c_{1} n_{0}-1\right)\right) \in \mathbb{Z}^{2}$ with $R \in$ CorPaths is bounded by $\left(\left|J_{1}\right|+c_{1} n_{0} l\right)^{2} \leq n_{0}^{41}$; recall that $n_{0}$ was chosen to be large (see Subsection 2.1). Furthermore, every such pair gives rise to at most a single element of Corrupted, since different paths $R, R^{\prime} \in$ CorPaths with the same starting point and the same end point generate the same word $\xi \circ R=\xi \circ R^{\prime}$ by Definition (8.46). This shows $\mid$ Corrupted $\mid \leq n_{0}^{41} \leq 4 \cdot 2^{n_{0}}$, since $n_{0}$ is large enough by Subsection 2.1. Using the definition of Center ${ }_{\text {II }}$, we obtain $\mid$ Center $_{\text {II }} \mid \leq 50 \cdot 2^{n_{0}}$.

Finally we show $\mid$ Center $_{\text {III }} \mid \geq 2^{n_{0} / 3}$. We observe that $\left[-2 \cdot 2^{n_{0} / 2}, 2 \cdot 2^{n_{0} / 2}\right] \backslash\left[-2^{n_{0} / 2}, 2^{n_{0} / 2}\right]$ is disjoint from $J_{1}$. Assuming that $B_{\text {unique fit }}^{n_{0}}\left(J_{1}\right)$ holds, this implies that all right ladder intervals $I_{1}, I_{2} \subseteq\left[-2 \cdot 2^{n_{0} / 2}, 2 \cdot 2^{n_{0} / 2}\right] \backslash\left[-2^{n_{0} / 2}, 2^{n_{0} / 2}\right]$, with $I_{1} \neq I_{2}\left|I_{1}\right|=\left|I_{2}\right|=c_{1} n_{0} \geq c_{2} n_{0}$ generate pairwise different ladder words $\left(\xi\left\lceil I_{1}\right) \rightarrow \neq\left(\xi\left\lceil I_{2}\right)_{\rightarrow}\right.\right.$. Since there are at least $2^{n_{0} / 2}-c_{1} n_{0} \geq 2^{n_{0} / 3}$ 
such ladder intervals ( $n_{0}$ is large enough; see Subsection 2.1), there are as least as many ladder words $w \in$ Center III.

The $T^{1}$ stopping times should not erroneously stop the random walk too far from the origin: If the random walk at time $t$ is at a location $x$ far away from the origin, we want the puzzle Puzzle IV $n_{\mathrm{IV}}\left(\theta^{t} \chi\right)$ collected there to have only a small overlap with the puzzle Puzzle $n_{\mathrm{IV}}^{n_{0}}(\chi)$ collected at the starting point, provided Puzzle $e_{\mathrm{IV}}^{n_{0}}\left(\theta^{t} \chi\right)$ is not anyway too large. The next lemma helps us to control this event, using the potential upper bound Center II of Puzzle ${ }_{\mathrm{IV}}^{n_{0}}(\chi)$.

Lemma 8.19 For every $x \in \mathbb{Z}$ with $|x|>2 \cdot 2^{n_{0}}+2 l 2^{12 \alpha n_{0}}$ and for every $t \in\left[0,2^{12 \alpha n_{1}}\right.$ [ it holds: $\tilde{P}\left[S(t)=x, \mid\right.$ Puzzle $_{\mathrm{IV}}^{n_{0}}\left(\theta^{t} \chi\right) \mid \leq 50 \cdot 2^{n_{0}}$, and $\mid$ Center $\left._{\mathrm{II}} \cap \operatorname{Puzzle}_{\mathrm{IV}}^{n_{0}}\left(\theta^{t} \chi\right) \mid \geq 2^{n_{0} / 3}\right] \leq \exp \left\{-2^{n_{0} / 4}\right\}$.

Proof. We set

$$
\text { Outside }_{x, t}:= \begin{cases}\text { Puzzle }_{\mathrm{IV}}^{n_{0}}\left(\theta^{t} \chi\right) & \text { if } S(t)=x \text { and } \mid \text { Puzzle }_{\mathrm{IV}}^{n_{0}}\left(\theta^{t} \chi\right) \mid \leq 50 \cdot 2^{n_{0}} \\ \emptyset & \text { else. }\end{cases}
$$

The random set Puzzle $e_{\mathrm{IV}}^{n_{0}}\left(\theta^{t} \chi\right)$ only depends on $\chi\left\lceil\left[t, t+2 \cdot 2^{12 \alpha n_{0}}[\right.\right.$, and the random walk cannot travel a longer distance than $2 l 2^{12 \alpha n_{0}}$ during the time interval $\left[t, t+2 \cdot 2^{12 \alpha n_{0}}[\right.$. Given $S(t)=x$ and $|x|>2 \cdot 2^{n_{0}}+2 l 2^{12 \alpha n_{0}}$, the random walk $S$ cannot enter the interval $\left[-2 \cdot 2^{n_{0}}, 2 \cdot 2^{n_{0}}\right]$ during the time interval $\left[t, t+2 \cdot 2^{12 \alpha n_{0}}\left[\right.\right.$; thus Outside $x, t$ depends only on $S$ and $\xi\left\lceil\left(\mathbb{Z} \backslash\left[-2 \cdot 2^{n_{0}}, 2 \cdot 2^{n_{0}}\right]\right)\right.$. Hence, using Lemma 8.1 and $J_{1} \subseteq\left[-2 \cdot 2^{n_{0}}, 2 \cdot 2^{n_{0}}\right]$, the random piece of scenery $\xi\left[\left[-2 \cdot 2^{n_{0}}, 2 \cdot 2^{n_{0}}\right]\right.$ and the random set Outside $x, t$ are independent with respect to $\tilde{P}$. Let $\mathcal{I}_{\mathrm{r}}$ denote the set of all right ladder intervals $I \subseteq\left[-2 \cdot 2^{n_{0}}, 2 \cdot 2^{n_{0}}\right] \backslash J_{1}$ with $|I|=c_{1} n_{0}$. We define $\mathcal{I}_{1}$ similarly with "right ladder intervals" replaced by "left ladder intervals". We partition $\mathcal{I}_{\mathrm{r}}$ into $c_{1} n_{0} l_{\rightarrow}$ subsets, $\mathcal{I}_{\mathrm{r}}^{\prime}(1), \ldots, \mathcal{I}_{\mathrm{r}}^{\prime}\left(c_{1} n_{0} l_{\rightarrow}\right)$ :

$$
\mathcal{I}_{\mathrm{r}}^{\prime}(k):=\left\{I \in \mathcal{I}_{\mathrm{r}} \mid \min I \in k+c_{1} n_{0} l_{\rightarrow} \mathbb{Z}\right\}
$$

Let $k \in\left[1, c_{1} n_{0} l_{\rightarrow}\right]$ be fixed. Note that the cardinality $N:=\left|\mathcal{I}_{\mathrm{r}}^{\prime}(k)\right|$ fulfills the bounds

$$
\frac{2^{n_{0}}}{c_{1} n_{0} l_{\rightarrow}} \leq \frac{4 \cdot 2^{n_{0}}}{c_{1} n_{0} l_{\rightarrow}}-\left|J_{1}\right|-2 c_{1} n_{0} \leq N \leq \frac{4 \cdot 2^{n_{0}}}{c_{1} n_{0} l_{\rightarrow}}
$$

Furthermore, the elements of $\mathcal{I}_{\mathrm{r}}^{\prime}(k)$ are pairwise disjoint; thus the family $\left(\xi\lceil I)_{I \in \mathcal{I}_{\mathrm{r}}^{\prime}(k)}\right.$ is i.i.d. and independent of Outside ${ }_{x, t}$ (with respect to $\tilde{P}$ ). For $I \in \mathcal{I}_{\mathrm{r}}$, we set $X_{I}^{\mathrm{r}}:=1$ for $\left(\xi\lceil I)_{\rightarrow} \in\right.$ Outside $_{x, t}$, and $X_{I}^{\mathrm{r}}:=0$ otherwise. Similarly for $J \in \mathcal{I}_{1}$, let $X_{J}^{\mathrm{l}}$ denote the indicator function of the event $\left\{\left(\xi\lceil J)_{\leftarrow} \in\right.\right.$ Outside $\left._{x, t}\right\}$. Then, conditioned on a given value of Outside ${ }_{x, t}$, the Bernoulli random variables $X_{I}^{\mathrm{r}}, I \in \mathcal{I}_{\mathrm{r}}^{\prime}(k)$, are i.i.d. with respect to $\tilde{P}\left[\cdot \mid\right.$ Outside $\left._{x, t}\right]$. Furthermore we have, using $\mid$ Outside $_{x, t} \mid \leq 50 \cdot 2^{n_{0}}$ :

$$
\tilde{P}\left[X_{I}^{\mathrm{r}}=1 \mid \text { Outside }_{x, t}\right] \leq \mid \text { Outside }\left._{x, t}|| \mathcal{C}\right|^{-|I|} \leq 50 e^{\left(\log 2-c_{1} \log |\mathcal{C}|\right) n_{0}}=: p .
$$

We set $Y_{k}^{\mathrm{r}}:=\sum_{I \in \mathcal{I}_{\mathrm{r}}^{\prime}(k)} X_{I}^{\mathrm{r}}$. Consequently this random variable is stochastically dominated by a $\operatorname{Binomial}(N, p)$-distributed random variable; note that $Y_{k}^{\mathrm{r}}$ is binomially distributed with respect to the conditional measure $\tilde{P}\left[\cdot \mid\right.$ Outside $\left._{x, t}\right]$. A rough but simple large deviation estimate suffices for our purposes: Using the exponential Chebyshev inequality, we have for $a>0$ and $\sigma:=\log (a / p)>0$ :

$$
\begin{aligned}
& \tilde{P}\left[Y_{k}^{\mathrm{r}} \geq N a\right] \leq E\left[e^{\sigma Y_{k}^{\mathrm{r}}-N a}\right] \leq\left(p e^{\sigma(1-a)}+(1-p) e^{-\sigma a}\right)^{N}=\left((1+a-p) p^{a} a^{-a}\right)^{N} \\
& \leq\left(e^{a} p^{a} a^{-a}\right)^{N}=\exp \{N a(1-\log (a / p))\}
\end{aligned}
$$


In particular, we obtain for the choice $a=N^{-1} 2^{n_{0} / 3} /\left(4 c_{1} n_{0} l_{\rightarrow}\right) \geq 2^{-2 n_{0} / 3} / 16$ (where we have used (8.56)), using (8.57): $\sigma=\log (a / p) \geq\left(c_{1} \log |\mathcal{C}|-\frac{5}{3} \log 2\right) n_{0}-\log 800 \geq c_{1}(\log |\mathcal{C}|) n_{0} / 2+1$; the last inequality holds by our choice of $c_{1}$ and $n_{0}$ (see Subsection 2.1). Hence we obtain:

$$
\begin{aligned}
& \tilde{P}\left[\sum_{I \in \mathcal{I}_{\mathrm{r}}} X_{I}^{\mathrm{r}} \geq \frac{2^{n_{0} / 3}}{4}\right] \leq \sum_{k=1}^{c_{1} n_{0} l_{\rightarrow}} \tilde{P}\left[Y_{k}^{\mathrm{r}} \geq N a\right] \leq c_{1} n_{0} l_{\rightarrow} \exp \{N a(1-\log (a / p))\} \\
& \leq c_{1} n_{0} l_{\rightarrow} \exp \left\{-\frac{\log |\mathcal{C}|}{8 l_{\rightarrow}} 2^{n_{0} / 3}\right\} \leq \frac{1}{2} \exp \left\{-2^{n_{0} / 4}\right\} .
\end{aligned}
$$

The same argument works for left ladder intervals, too:

$$
\tilde{P}\left[\sum_{J \in \mathcal{I}_{1}} X_{J}^{1} \geq \frac{2^{n_{0} / 3}}{4}\right] \leq \frac{1}{2} \exp \left\{-2^{n_{0} / 4}\right\} .
$$

We know $\mid$ Corrupted $\mid \leq n_{0}^{41} \leq 2^{n_{0} / 3} / 2$ (see Lemma 8.18), and hence

$$
\mid \text { Center }_{\text {II }} \cap \text { Outside }_{x, t}|\leq| \text { Center }_{\mathrm{I}} \cap \text { Outside }_{x, t}|+| \text { Corrupted }|\leq| \text { Center }_{\mathrm{I}} \cap \text { Outside }_{x, t} \mid+\frac{2^{n_{0} / 3}}{2}
$$

by Definition (8.49) of Center II. Combining this with (8.60) and (8.59), we obtain

$$
\begin{aligned}
& \tilde{P}\left[\mid \text { Center }_{\text {II }} \cap \text { Outside }_{x, t} \mid \geq 2^{n_{0} / 3}\right] \leq \tilde{P}\left[\mid \text { Center }_{\mathrm{I}} \cap \text { Outside }_{x, t} \mid \geq \frac{2^{n_{0} / 3}}{2}\right] \\
& \leq \tilde{P}\left[\sum_{I \in \mathcal{I}_{\mathrm{r}}} X_{I}^{\mathrm{r}} \geq \frac{2^{n_{0} / 3}}{4}\right]+\tilde{P}\left[\sum_{J \in \mathcal{I}_{1}} X_{J}^{\mathrm{l}} \geq \frac{2^{n_{0} / 3}}{4}\right] \leq \exp \left\{-2^{n_{0} / 4}\right\} .
\end{aligned}
$$

The claim (8.53) is an immediate consequence of this bound and the Definition (8.54) of Outside $_{x, t}$.

The following is a modified version of Lemma 6.37. To this end it may be useful to recall Definition (6.21) of the event $B_{\text {recogn straight }}^{n_{0}}\left(J_{1}\right)$. Up to a possible perturbation by $J_{1}$, this event describes that ladder paths can be distinguished from non-ladder paths by some uniqueness property of their color record.

Lemma 8.20 There exist positive constants $c_{25}$ and $c_{26}$ not depending on $n_{0}$ such that:

$$
\tilde{P}\left[\left(B_{\text {recogn straight }}^{n_{0}}\left(J_{1}\right)\right)^{\mathrm{c}}\right] \leq c_{25} e^{-c_{26} n_{0}} .
$$

Proof. The main difference between Lemma 6.37 and Lemma 8.20 is the usage of the modified measure $\tilde{P}$ instead of $P$. However, the event $B_{\text {recogn straight }}^{n_{0}}\left(J_{1}\right)$, introduced in definition (6.21), only depends on the part $\xi\left\lceil\left(\mathbb{Z} \backslash J_{1}\right)\right.$ of the scenery outside $J_{1}$. Indeed: Given $R \in \operatorname{AdPaths}(11$. $\left.2^{n_{0}}, c_{1} n_{0}\right)$ with $R\left(c_{1} n_{0}-1\right)-R(0) \notin\left\{\left(c_{1} n_{0}-1\right) l_{\rightarrow},\left(c_{1} n_{0}-1\right) l_{\leftarrow}\right\}$, there are two cases: Either some $\bar{R} \in \operatorname{AdPaths}\left(12 \cdot 2^{n_{0}}, c_{1} n_{0}\right)$ with $\bar{R}(0)=R(0)$ and $\bar{R}\left(c_{1} n_{0}-1\right)=R\left(c_{1} n_{0}-1\right)$ takes at least one value in $J_{1}$ ("case 1 "), or no such $\bar{R}$ touches $J_{1}$ ("case 2 ").

- In case 1 , we do not need to evaluate $\xi \circ R$ of $\xi \circ \bar{R}$ in order to check the defining condition (6.21) of $B_{\text {recogn straight }}^{n_{0}}\left(J_{1}\right)$. 
- In case $2, \xi \circ R$ and $\xi \circ \bar{R}$ depend only on $\xi\left\lceil\left(\mathbb{Z} \backslash J_{1}\right)\right.$.

On the other hand, the distributions of $\xi\left\lceil\left(\mathbb{Z} \backslash J_{1}\right)\right.$ with respect to $P$ and with respect to $\tilde{P}$ are the same by Lemma 8.1. Thus

$$
\tilde{P}\left[\left(B_{\text {recogn straight }}^{n_{0}}\left(J_{1}\right)\right)^{c}\right]=P\left[\left(B_{\text {recogn straight }}^{n_{0}}\left(J_{1}\right)\right)^{c}\right] \leq c_{25} e^{-c_{26} n_{0}}
$$

follows from Lemma 6.37. This proves the claim (8.63).

We need the following modification of Definition 6.18:

\section{Definition 8.21}

$$
\tilde{E}_{\text {only ladder }}^{n_{0}}:=\left\{\begin{array}{l}
\text { For all }\left(w_{1}, w_{2}, w_{3}\right) \in \text { Puzzle }_{\mathrm{I}}^{n_{0}}\left(\text { Input }_{0}\right) \text { and every admissible piece } \\
\text { of path } R:\left[0,3 c_{1} n_{0}\left[\rightarrow\left[-11 \cdot 2^{n_{0}}, 11 \cdot 2^{n_{0}}\right] \text { with } \xi \circ R=w_{1} w_{2} w_{3}\right.\right. \\
\text { holds: } w_{2} \text { is a ladder word of } \xi\left[\left[-11 \cdot 2^{n_{0}}, 11 \cdot 2^{n_{0}}\right], \text { or } w_{2} \in\right. \\
\text { Corrupted. }
\end{array}\right\} .
$$

Here is an analogue of Lemma 6.19; recall the definition (6.9) of the events $B_{\text {all paths, } \tau}^{n}$ :

Lemma 8.22 We have

$$
B_{\text {all paths }, T^{0}}^{n_{0}} \cap B_{\text {recogn straight }}^{n_{0}}\left(J_{1}\right) \subseteq \tilde{E}_{\text {only ladder }}^{n_{0}} .
$$

Proof. The proof is partially similar to the proof of Lemma 6.19. Assume the event $B_{\text {all paths, } T^{0}}^{n_{0}} \cap$ $B_{\text {recogn straight }}^{n_{0}}\left(J_{1}\right)$ holds, and let $\left(w_{1}, w_{2}, w_{3}\right) \in \operatorname{Puzzle}_{\mathrm{I}}^{n_{0}}\left(\right.$ Input $\left._{0}\right), R \in \operatorname{AdPaths}\left(11 \cdot 2^{n_{0}}, 3 c_{1} n_{0}\right)$ with $\xi \circ R=w_{1} w_{2} w_{3}$. We prove by contradiction that $\tilde{E}_{\text {only ladder }}^{n_{0}}$ holds: Assume that $w_{2}$ is not a ladder word of $\xi\left\lceil\left[-11 \cdot 2^{n_{0}}, 11 \cdot 2^{n_{0}}\right]\right.$ and $w_{2} \notin$ Corrupted. We distinguish two cases: Either the middle piece $R\left\lceil\left[c_{1} n_{0}, 2 c_{1} n_{0}\right.\right.$ [ of $R$ belongs to CorPaths when being time-shifted back to the origin ("case 1"), or it does not ("case 2").

- In case $1, w_{2}=\left(\xi \circ R\left\lceil\left[c_{1} n_{0}, 2 c_{1} n_{0}[)_{\rightarrow} \in\right.\right.\right.$ Corrupted by Definition (8.47), which contradicts our assumption.

- In case 2, using Definition (8.46) of CorPaths, there is an admissible piece of path $R^{\prime}$ : $\left[c_{1} n_{0}, 2 c_{1} n_{0}\left[\rightarrow \mathbb{Z}\right.\right.$ with $R^{\prime}\left(c_{1} n_{0}\right)=R\left(c_{1} n_{0}\right)$ and $R^{\prime}\left(2 c_{1} n_{0}-1\right)=R\left(2 c_{1} n_{0}-1\right)$ such that $w_{2}^{\prime}:=\left(\xi \circ R^{\prime}\right) \rightarrow \neq\left(\xi \circ R\left\lceil\left[c_{1} n_{0}, 2 c_{1} n_{0}[) \rightarrow\right.\right.\right.$ ("case 2.1"), or all admissible paths $R^{\prime}:\left[c_{1} n_{0}, 2 c_{1} n_{0}\left[\rightarrow \mathbb{Z}\right.\right.$ with $R^{\prime}\left(c_{1} n_{0}\right)=R\left(c_{1} n_{0}\right)$ and $R^{\prime}\left(2 c_{1} n_{0}-1\right)=R\left(2 c_{1} n_{0}-1\right)$ do not touch $J_{1}$ and fulfill $\xi \circ R^{\prime}=\xi \circ R\left\lceil\left[c_{1} n_{0}, 2 c_{1} n_{0}\right.\right.$ [ ("case 2.2 ").

- In case 2.1, we take a path $R^{\prime}$ with the properties mentioned above. Let $\check{R}$ : $\left[0,3 c_{1} n_{0}\left[\rightarrow\left[-11 \cdot 2^{n_{0}}, 11 \cdot 2^{n_{0}}\right]\right.\right.$ be the admissible piece of path which on $\left[c_{1} n_{0}, 2 c_{1} n_{0}[\right.$ is equal to $R^{\prime}$ and otherwise is equal to $R$. We have $\xi \circ \check{R}=w_{1} w_{2}^{\prime} w_{3}$. Since $B_{\text {all paths }, T^{0}}^{n_{0}}$ holds (recall its definition (6.9)), this implies that the random walk $S$ follows the path of $\check{R}$ within time $2^{2 n_{0}}$ from a stopping time of $T_{k}^{0}, k<2^{\alpha n_{0}}$. The same is valid for $R$, maybe with a different stopping time $T_{k^{\prime}}^{0}$. In other words: $\left(w_{1}, w_{2}^{\prime}, w_{3}\right) \in \operatorname{PrePuzzle}^{n_{0}}\left(\right.$ Input $\left._{0}\right)$ and $\left(w_{1}, w_{2}, w_{3}\right) \in$ PrePuzzle $^{n_{0}}\left(\right.$ Input $\left._{0}\right)$. This implies the contradiction $\left(w_{1}, w_{2}, w_{3}\right) \notin \operatorname{Puzzle}_{\mathrm{I}}^{n_{0}}\left(\operatorname{Input}_{0}\right)$.

- In case 2.2 , we use that $R\left\lceil\left[c_{1} n_{0}, 2 c_{1} n_{0}\left[\right.\right.\right.$ is not a ladder path, since $w_{2}$ is not a ladder word of $\xi\left\lceil\left[-11 \cdot 2^{n_{0}}, 11 \cdot 2^{n_{0}}\right]\right.$. This case contradicts the occurrence of the event $B_{\text {recogn straight }}^{n_{0}}\left(J_{1}\right)$, using the definition (6.21) of this event and the defining condition of case 2.2 . 
Thus all cases lead to a contradiction; this proves the Lemma.

Roughly speaking, next lemma shows that Puzzle IV collects ladder words on a length scale $O\left(2^{n_{0}}\right)$ away from the origin, only perturbed by a few "corrupted" words, provided the "typical" events $\tilde{E}_{\text {only ladder }}^{n_{0}}$ and $E_{\text {stop }, T^{0}}^{0}$ hold. Formally, the statement gets stronger by using $E_{\text {stop }, T^{0}}^{0}$ rather than the smaller event $\tilde{E}_{\text {stop }, T^{0}}^{0}$; but this fact is not essential.

However, it is essential to use Puzzle $n_{I I I}^{n_{0}}$ or Puzzle ${ }_{\text {IV }}^{n_{0}}$ rather than Puzzle $\mathrm{I}_{\mathrm{I}}^{n_{0}}$ or Puzzle $\mathrm{e}_{\mathrm{II}}^{n_{0}}$ : We can be sure that Puzzle $n_{\mathrm{IV}}^{n_{0}}$ collects its words at most in a neighborhood of the origin of size $O\left(2^{n_{0}}\right)$, provided that the stopping times $T^{0}$ fulfill their specification, i.e. provided the event $E_{\text {stop, } T^{0}}^{0}$ holds. On the other hand, Puzzle II may collect words up to the length scale $O\left(2^{2 n_{0}}\right)$ away from the origin, even if the stopping times $T^{0}$ fulfill their specification. Recall that for Puzzle $\mathrm{II}_{\mathrm{I}}^{n_{0}}$, words are collected on a time scale $O\left(2^{2 n_{0}}\right)$ away from the stopping times $T^{0}$. Here, it does not help us that Puzzle $n_{I I}^{n_{0}}$ still typically collects words on a length scale $O\left(2^{n_{0}}\right)$ away from the origin, due to the scaling in the central limit theorem. To see this, recall that we are searching for overlaps $\left|\operatorname{Puzzle}_{\mathrm{IV}}^{n_{0}}(\chi) \cap \operatorname{Puzzle}_{\mathrm{IV}}^{n_{0}}\left(\theta^{t} \chi\right)\right|$ of the small size $2^{n_{0} / 3}$ only. If we were using Puzzle $\mathrm{II}$ rather than Puzzle IV , such an overlap could arise in the tails, and we had to deal with the length scale $O\left(2^{2 n_{0}}\right)$, which is much too large.

Lemma 8.23 If $\tilde{E}_{\text {only ladder }}^{n_{0}} \cap E_{\text {stop }, T^{0}}^{0}$ holds, then Puzzle $\mathrm{IV}_{\mathrm{IV}}(\chi) \subseteq$ Center $_{\mathrm{II}}$.

Proof. Assume that $\tilde{E}_{\text {only ladder }}^{n_{0}} \cap E_{\text {stop } T^{0}}^{0}$ holds, and let $w_{2} \in \operatorname{Puzzle}_{\mathrm{IV}}^{n_{0}}(\chi)$. Take $w_{1}, w_{3} \in \mathcal{C}^{c_{1} n_{0}}$ with $\left(w_{1}, w_{2}, w_{3}\right) \in$ Puzzle III $_{\text {III }}^{n_{0}} \chi$ by $(8.43)$. Then by $(8.42),\left(w_{1}, w_{2}, w_{3}\right) \in$ Puzzle $_{\mathrm{I}}^{n_{0}}\left(\right.$ Input $\left._{0}\right)$, and $w_{1} w_{2} w_{3}$ occurs in the observations $\chi$ at most $2^{n_{0}} / l$ time steps after some stopping time $T_{k}^{0}(\chi), 0 \leq k<2^{\alpha n_{0}}$. Since $E_{\mathrm{stop}, T^{0}}^{0}$ holds, we have $\left|S\left(T_{k}^{0}\right)\right| \leq 2^{n_{0}}$; thus $w_{1} w_{2} w_{3}$ is read in $\chi$ while the random walk follows some admissible piece of path $R$ with values in $\left[-2 \cdot 2^{n_{0}}, 2 \cdot 2^{n_{0}}\right] \subseteq$ $\left[-11 \cdot 2^{n_{0}}, 11 \cdot 2^{n_{0}}\right]$. Since $\tilde{E}_{\text {only ladder }}^{n_{0}}$ holds, this implies: $w_{2}$ is a ladder word of $\xi\left\lceil\left[-11 \cdot 2^{n_{0}}, 11 \cdot 2^{n_{0}}\right]\right.$, or $w_{2} \in$ Corrupted. If $w_{2}$ is a ladder word of $\xi\left\lceil\left[-11 \cdot 2^{n_{0}}, 11 \cdot 2^{n_{0}}\right]\right.$, we distinguish two cases:

- If $w_{2}$ is a ladder word of $\xi\left\lceil\left(\left[-11 \cdot 2^{n_{0}}, 11 \cdot 2^{n_{0}}\right] \backslash J_{1}\right)\right.$, then $w_{2} \in$ Center I.

- If $w_{2}$ is a ladder word of $\xi\left\lceil\left[-11 \cdot 2^{n_{0}}, 11 \cdot 2^{n_{0}}\right]\right.$, but not of $\xi\left\lceil\left(\left[-11 \cdot 2^{n_{0}}, 11 \cdot 2^{n_{0}}\right] \backslash J_{1}\right)\right.$, then also $w_{2} \in$ Corrupted. To see this, we use the Definitions (8.46) and (8.47), and the following fact: If $\pi$ is a ladder path and $\bar{\pi}$ is an admissible piece of path with the same length, starting point, and end point as $\pi$, then $\bar{\pi}=\pi$.

In any case, we have $w_{2} \in$ Center $_{\mathrm{I}} \cup$ Corrupted $=$ Center $_{\mathrm{II}}$.

Next we will show that the events we defined have sufficiently large probability.

Lemma 8.24 There exist constants $c_{20}, c_{21}>0$ such that:

$$
\tilde{P}\left[\left(B_{\text {all paths }, T^{0}}^{n_{0}}\right)^{\mathrm{c}} \cap E_{\text {stop }, T^{0}}^{0}\right] \leq c_{21} e^{-c_{20} n_{0}} .
$$

Proof. This is a special case of Lemma 6.34, applied to $\tau=T^{0}$ and $P^{\prime}=\tilde{P}$. The hypothesis of Lemma 6.34 is fulfilled, since part 2 in Lemma 8.1 implies that the distribution of $S$ with respect to $P$ and with respect to $\tilde{P}$ coincide.

Recall Definition (7.1): $E_{\text {no error, } T^{1}}^{1}=\left\{\forall k \in \mathbb{N}\right.$ : If $T_{k}^{1}(\chi)<2^{12 \alpha n_{1}}$, then $\left.\left|S\left(T_{k}^{1}(\chi)\right)\right| \leq 2^{n_{1}}\right\}$.

Lemma 8.25 For some constants $c_{48}, c_{49}>0$ holds $\tilde{P}\left[E_{\text {no error, } T^{1}}^{1}\right] \geq 1-c_{48} e^{-c_{49} n_{0}}$. 
Proof. Using Definition 8.15 of $T^{1}$, we obtain:

$$
\begin{aligned}
& \tilde{P}\left[E_{\text {no error, } T^{1}}^{1}\right] \\
& \geq \tilde{P}\left[\begin{array}{l}
\text { For all } t \in\left[0,2^{12 \alpha n_{1}}\left[\text { it holds: if } \mid \text { Puzzle } \operatorname{IV}_{0}^{n_{0}}(\chi) \cap \text { Puzzle }_{\mathrm{IV}}^{n_{0}}\left(\theta^{t} \chi\right) \mid \geq 2^{n_{0} / 3}\right. \text { and }\right. \\
\mid \text { Puzzle }_{\mathrm{IV}}^{n_{0}}\left(\theta^{t} \chi\right) \mid \leq 50 \cdot 2^{n_{0}}, \text { then }\left|S\left(t+2^{12 \alpha n_{0}}\right)\right| \leq 2^{n_{1}}
\end{array}\right]
\end{aligned}
$$

For $i=0,1$, the random walk $S$ cannot travel farther than $l 2^{12 \alpha n_{i}}$ within time $2^{12 \alpha n_{i}}$. Since $n_{0}$ is large (recall Subsection 2.1) and $n_{1}=2^{\left\lfloor\sqrt{n_{0}}\right\rfloor}$, we know $2 \cdot 2^{n_{0}}+2 l 2^{12 \alpha n_{0}}<2^{n_{1}}-l 2^{12 \alpha n_{0}}$. Thus for all $t \in\left[0,2^{12 \alpha n_{1}}\left[\right.\right.$, we have $\left|S\left(t+2^{12 \alpha n_{0}}\right)\right| \leq 2^{n_{1}}$ or $2 \cdot 2^{n_{0}}+2 l 2^{12 \alpha n_{0}}<|S(t)| \leq l 2^{12 \alpha n_{1}}$; recall that $S(0)=0$. Thus the right hand side in (8.68) is greater than or equal to

$$
\begin{aligned}
& \tilde{P}\left[\begin{array}{l}
\text { There does not exist } t \in\left[0,2^{12 \alpha n_{1}}\left[\text {, such that } \mid \text { Puzzle }_{\mathrm{IV}}^{n_{0}}(\chi) \cap \text { Puzzle }_{\mathrm{IV}}^{n_{0}}\left(\theta^{t} \chi\right) \mid \geq 2^{n_{0} / 3}\right.\right. \\
\text { and } \mid \text { Puzzle }_{\mathrm{IV}}^{n_{0}}\left(\theta^{t} \chi\right) \mid \leq 50 \cdot 2^{n_{0}} \text { and } 2 \cdot 2^{n_{0}}+2 l 2^{12 \alpha n_{0}}<|S(t)| \leq l 2^{12 \alpha n_{1}}
\end{array}\right] \\
& \geq \tilde{P}\left[\begin{array}{l}
\operatorname{Puzzle}_{\mathrm{IV}}^{n_{0}}(\chi) \subseteq \text { Center }_{\mathrm{II}}, \text { and there does not exist } t \in\left[0,2^{12 \alpha n_{1}}[\text {, such that }\right. \\
\mid \text { Center }_{\mathrm{II}} \cap \text { Puzzle }_{\mathrm{IV}}^{n_{0}}\left(\theta^{t} \chi\right) \mid \geq 2^{n_{0} / 3} \text { and } \mid \text { Puzzle }_{\mathrm{IV}}^{n_{0}}\left(\theta^{t} \chi\right) \mid \leq 50 \cdot 2^{n_{0}} \text { and } \\
2 \cdot 2^{n_{0}}+2 l 2^{12 \alpha n_{0}}<|S(t)| \leq l 2^{12 \alpha n_{1}}
\end{array}\right] \\
& \geq \tilde{P}\left[\text { Puzzle }_{\mathrm{IV}}^{n_{0}}(\chi) \subseteq \text { Center }_{\mathrm{II}}\right]-\sum_{t=0}^{2^{12 \alpha n_{1}}-1} \tilde{P}\left[\begin{array}{l}
\mid \text { Center }_{\mathrm{II}} \cap \text { Puzzle }_{\mathrm{IV}}^{n_{0}}\left(\theta^{t} \chi\right) \mid \geq 2^{n_{0} / 3}, \\
\mid \text { Puzzle }_{\mathrm{IV}}^{n_{0}}\left(\theta^{t} \chi\right) \mid \leq 50 \cdot 2^{n_{0}}, \text { and } \\
2 \cdot 2^{n_{0}}+2 l 2^{12 \alpha n_{0}}<|S(t)| \leq l 2^{12 \alpha n_{1}}
\end{array}\right] .
\end{aligned}
$$

Using Lemma 8.19, each summand in the last sum has the upper bound $2 l 2^{12 \alpha n_{1}} \cdot \exp \left\{-2^{n_{0} / 4}\right\}$; note that there are at most $2 l 2^{12 \alpha n_{1}}$ possible values for $x=S(t)$ with $2 \cdot 2^{n_{0}}+2 l 2^{12 \alpha n_{0}}<|x| \leq$ $l 2^{12 \alpha n_{1}}$. Furthermore, the occurrence of the events $E_{\text {stop }, T^{0}}^{0}, B_{\text {recogn straight }}^{n_{0}}\left(J_{1}\right)$ and $B_{\text {all paths, } T^{0}}^{n_{0}}$ implies Puzzle $e_{\mathrm{IV}}^{n_{0}}(\chi) \subseteq$ Center $_{\mathrm{II}}$ by Lemma 8.22 and Lemma 8.23. Thus the right hand side in (8.69) has the lower bound

$$
\begin{aligned}
& \tilde{P}\left[E_{\text {stop }, T^{0}}^{0} \cap B_{\text {recogn straight }}^{n_{0}}\left(J_{1}\right) \cap B_{\text {all paths }, T^{0}}^{n_{0}}\right]-2^{12 \alpha n_{1}} \cdot 2 l 2^{12 \alpha n_{1}} \cdot \exp \left\{-2^{n_{0} / 4}\right\} \\
& \geq \tilde{P}\left[E_{\text {stop }, T^{0}}^{0}\right]-\tilde{P}\left[\left(B_{\text {recogn straight }}^{n_{0}}\left(J_{1}\right)\right)^{\mathrm{c}}\right] \\
& \quad-\tilde{P}\left[\left(B_{\text {all paths }, T^{0}}^{n_{0}}\right)^{\mathrm{c}} \cap E_{\text {stop }, T^{0}}^{0}\right]-2 l \exp \left\{24(\log 2) \alpha n_{1}-2^{n_{0} / 4}\right\} .
\end{aligned}
$$

We estimate the last expression, using Theorem 8.5, Lemmas 8.20 and 8.24, and the fact $n_{1}=$ $2^{\left\lfloor\sqrt{n_{0}}\right\rfloor}$ (recall Definition 3.4). We obtain the following lower bound for the right hand side in (8.70):

$$
1-c_{36} e^{-c_{37} n_{0}}-c_{25} e^{-c_{26} n_{0}}-c_{21} e^{-c_{20} n_{0}}-2 l \exp \left\{24(\log 2) \alpha 2^{\left\lfloor\sqrt{n_{0}}\right\rfloor}-2^{n_{0} / 4}\right\} \geq 1-c_{48} e^{-c_{49} n_{0}} .
$$

For the last step, note that $\exp \left\{24(\log 2) \alpha 2^{\left\lfloor\sqrt{n_{0}}\right\rfloor}-2^{n_{0} / 4}\right\}$ is superexponentially small in $n_{0}$. The constants $c_{48}$ and $c_{49}$ need not depend on $\alpha$, since $n_{0}$ was chosen large and $\alpha$-dependent (see Subsection 2.1).

The following definition introduces a variant $B_{\text {all paths II }}^{n_{0}}$ of the event $B_{\text {all paths, } T^{0}}^{n_{0}}$. Instead of the time scale $O\left(2^{2 n_{0}}\right)$ after a stopping time, it uses the time scale $O\left(2^{n_{0}}\right)$, and instead of the length scale $O\left(2^{n_{0}}\right)$, it uses the length scale $O\left(2^{n_{0} / 2}\right)$. We need to use the new event $B_{\text {all paths II }}^{n_{0}}$ rather than using exclusively the old event $B_{\text {all paths, } T^{0}}^{n_{0}}$, since we have to deal with Puzzle IV rather than using Puzzle II again, as was explained above: We need to control whether the random walk follows sufficiently many admissible paths within the shorter time horizon $O\left(2^{n_{0}}\right)$ after a $T^{0}$-stopping time. 
Definition 8.26 We define the event

$$
B_{\text {all paths II }}^{n_{0}}:=\left\{\begin{array}{l}
\forall R \in \operatorname{AdPaths}\left(3 \cdot 2^{n_{0} / 2}, 3 c_{1} n_{0}\right) \exists k \in\left[0,2^{\alpha n_{0}}\left[\exists j \in\left[0,2^{n_{0}} / l\right]:\right.\right. \\
\operatorname{TimeShift}_{k}^{T^{0}}(\chi)+j \\
\operatorname{mim}(R) \subseteq S
\end{array}\right\} .
$$

The next lemma provides yet another modification of Lemma 6.34. Up to a change of parameters, its proof is very similar to the proof of Lemma 6.34 .

Lemma 8.27 There exist constants $c_{50}, c_{51}>0$ not depending on $n_{0}$ such that:

$$
\tilde{P}\left[\left(B_{\text {all paths II }}^{n_{0}}\right)^{\mathrm{c}} \cap \tilde{E}_{\text {stop }, T^{0}}^{0}\right] \leq c_{50} e^{-c_{51} n_{0}} .
$$

Here, it is important to use the stricter specification $\tilde{E}_{\text {stop }, T^{0}}^{0}$ of the stopping times $T^{0}$ rather than $E_{\mathrm{stop}, T^{0}}^{0}$ : Knowing the location of the random walker only on a scale $O\left(2^{n_{0}}\right)$ rather than $O\left(2^{n_{0} / 2}\right)$ will not suffice: Within $O\left(2^{n_{0}}\right)$ after a stopping time, the random walker will typically move only a distance $O\left(2^{n_{0} / 2}\right)$ and thus might be still too far from the origin, unless we assume the stricter bound $\left|S\left(T_{k}^{0}\right)\right|=O\left(2^{n_{0} / 2}\right)$.

Proof of Lemma 8.27. Let $k<2^{\alpha n_{0}}$ and $R \in \operatorname{AdPaths}\left(3 \cdot 2^{n_{0} / 2}, 3 c_{1} n_{0}\right)$. We set

$$
\begin{aligned}
& B_{R}^{n_{0}, k}:=\left\{\exists j \in\left[0,2^{n_{0}} / l\right]: \text { TimeShift } T_{k}^{0}+j(R) \subseteq S\right\} \\
& \tilde{E}_{\text {stop }, T^{0}, k}^{0}:=\left\{\begin{array}{l}
T_{k}^{0}(\chi)<2^{12 \alpha n_{0}},\left|S\left(T_{k}^{0}(\chi)\right)\right| \leq 2^{n_{0} / 2}, \\
T_{j}^{0}(\chi)+2 \cdot 2^{2 n_{0}} \leq T_{k}^{0}(\chi) \text { for } j<k
\end{array}\right\}, \\
& A_{R}^{n_{0}, k}:=\tilde{E}_{\mathrm{stop}, T^{0}, k}^{0} \backslash B_{R}^{n_{0}, k} .
\end{aligned}
$$

In the following calculations, $R$ runs over the set $\operatorname{AdPaths}\left(3 \cdot 2^{n_{0} / 2}, 3 c_{1} n_{0}\right)$ :

$$
\begin{aligned}
& \tilde{E}_{\text {stop }, T^{0}}^{0} \backslash B_{\text {all paths II }, T^{0}}^{n_{0}}=\left(\bigcap_{k=0}^{2^{\alpha n_{0}}} \tilde{E}_{\text {stop }, T^{0}, k}^{0}\right) \backslash\left(\bigcap_{R} \bigcup_{k=0}^{2^{\alpha n_{0}}-1} B_{R}^{n_{0}, k}\right) \\
& =\bigcup_{R} \bigcap_{k=0}^{2^{\alpha n_{0}-1}}\left[\left(\bigcap_{k^{\prime}=0}^{2^{\alpha n_{0}}} \tilde{E}_{\mathrm{stop}, T^{0}, k^{\prime}}^{0}\right) \backslash B_{R}^{n_{0}, k}\right] \subseteq \bigcup_{R} \bigcap_{k=0}^{2^{\alpha n_{0}-1}} A_{R}^{n_{0}, k} .
\end{aligned}
$$

Taking the probability of these events, we estimate:

$$
\begin{aligned}
& \tilde{P}\left[\left(B_{\text {all paths II }, T^{0}}^{n_{0}}\right)^{\mathrm{c}} \cap \tilde{E}_{\text {stop }, T^{0}}^{0}\right] \leq\left|\operatorname{AdPaths}\left(3 \cdot 2^{n_{0} / 2}, 3 c_{1} n_{0}\right)\right| \max _{R} \tilde{P}\left[\bigcap_{k=0}^{2^{\alpha n_{0}}-1} A_{R}^{n_{0}, k}\right], \\
& \left|\operatorname{AdPaths}\left(3 \cdot 2^{n_{0} / 2}, 3 c_{1} n_{0}\right)\right| \leq 7 \cdot 2^{n_{0} / 2}|\mathcal{M}|^{3 c_{1} n_{0}}, \\
& \tilde{P}\left[\bigcap_{k=0}^{2^{\alpha n_{0}}-1} A_{R}^{n_{0}, k}\right]=\prod_{k=0}^{2^{\alpha n_{0}-1}} \tilde{P}\left[A_{R}^{n_{0}, k} \mid \bigcap_{j<k} A_{R}^{n_{0}, j}\right], \\
& \tilde{P}\left[A_{R}^{n_{0}, k} \mid \bigcap_{j<k} A_{R}^{n_{0}, j}\right] \leq \tilde{P}\left[\left(B_{R}^{n_{0}, k}\right)^{\mathrm{c}} \mid \tilde{E}_{\mathrm{stop}, T^{0}, k}^{0} \cap \bigcap_{j<k} A_{R}^{n_{0}, j}\right] ;
\end{aligned}
$$

the last statement follows from the elementary fact $\tilde{P}[A \cap B \mid C] \leq \tilde{P}[A \mid B \cap C]$. We have $C_{R}^{n_{0}, k}:=\tilde{E}_{\text {stop }, T^{0}, k}^{0} \cap \bigcap_{j<k} A_{R}^{n_{0}, j} \in \mathcal{F}_{T_{k}^{0}}$, i.e. one can decide whether the event $C_{R}^{n_{0}, k}$ holds by 
observing $\xi$ and $S(0), \ldots, S\left(T_{k}^{0}\right)$. Furthermore, if $C_{R}^{n_{0}, k}$ holds, then $\left|S\left(T_{k}^{0}(\chi)\right)\right| \leq 2^{n_{0} / 2}$. As consequence of the local Central Limit Theorem we get: there is a constant $c_{22}>0$ such that for all $x, y$ with $|x| \leq 3 \cdot 2^{n_{0} / 2}$ and $|y| \leq 2^{n_{0} / 2}$ one has

$$
\tilde{P}\left[y+S(j)=x \text { for some } j \in\left[0,2^{n_{0}} / l-3 c_{1} n_{0}\right]\right] \geq c_{22} 2^{-n_{0} / 2} ;
$$

note that $2^{n_{0}} / l-3 c_{1} n_{0}$ has the order of magnitude $2^{n_{0}}$. Thus by the strong Markov property:

$$
\inf _{|x| \leq 3 \cdot 2^{n_{0} / 2}} \tilde{P}\left[S\left(T_{k}^{0}+j\right)=x \text { for some } j \in\left[0,2^{n_{0}} / l\right] \mid C_{R}^{n_{0}, k}\right] \geq c_{52} 2^{-n_{0} / 2}
$$

for some constant $c_{52}>0$. Once it is in the starting point $x$, the probability that $S$ follows an admissible path $R \in \operatorname{AdPaths}\left(3 \cdot 2^{n_{0} / 2}, 3 c_{1} n_{0}\right)$ for the next $3 c_{1} n_{0}-1$ steps is bounded from below by $\mu_{\min }^{3 c_{1} n}$. Here $\mu_{\min }:=\min \{\mu(\{x\}) \mid x \in \mathcal{M}\}$ is the smallest positive probability for a jump. Therefore, using the strong Markov property again:

$$
\tilde{P}\left[B_{R}^{n_{0}, k} \mid C_{R}^{n_{0}, k}\right] \geq c_{52} 2^{-n_{0} / 2} \mu_{\min }^{3 c_{1} n_{0}} .
$$

We combine (8.78)-(8.81) and (8.84) to obtain

$$
\begin{aligned}
& \tilde{P}\left[\tilde{E}_{\text {stop }, T^{0}}^{0} \backslash B_{\text {all paths II }}^{n_{0}}\right] \leq 7 \cdot 2^{n_{0} / 2}|\mathcal{M}|^{3 c_{1} n_{0}}\left(1-c_{52} 2^{-n_{0} / 2} \mu_{\min }^{3 c_{1} n_{0}}\right)^{2^{\alpha n_{0}}} \\
& \leq 7 \exp \left\{n_{0}\left(\frac{\log 2}{2}+3 c_{1} \log |\mathcal{M}|\right)-c_{52} e^{n_{0}\left(\alpha \log 2+3 c_{1} \log \mu_{\min }-(\log 2) / 2\right)}\right\}
\end{aligned}
$$

where we used $1-x \leq e^{-x}$ for $x=c_{52} 2^{-n_{0} / 2} \mu_{\text {min }}^{3 c_{1} n_{0}} \leq 1$. Now $\alpha \log 2+3 c_{1} \log \mu_{\text {min }}-(\log 2) / 2>0$ by our choice of $\alpha$ in Subsection 2.1; thus the right hand side of the last inequality converges to 0 superexponentially fast as $n_{0} \rightarrow \infty$. Note that we may choose an upper bound $c_{50} e^{-c_{51} n_{0}}$ for the right hand side in 8.85, where neither $c_{50}$ nor $c_{51}$ depend on $\alpha$ or $c_{1}$. This is true since $n_{0}$ was chosen large enough, depending on $c_{1}$ and $\alpha$; recall Subsection 2.1. This proves the lemma.

Next we modify Definition 6.4 to define two new events. Roughly speaking, these events measure whether within a certain space horizon but outside the "corrupted" region $J_{1}$, any ladder word can be read along admissible paths only roughly at one single location, thus making ladder words very characteristic for their location.

\section{Definition 8.28}

$$
\begin{aligned}
& \tilde{B}_{\text {signals }}^{n_{0}}:=\left\{\begin{array}{l}
\text { For every right ladder path } \pi \in\left(\left[-2 l 2^{2 n_{0}}, 2 l 2^{2 n_{0}}\right] \backslash J_{1}\right]^{\left[0, c_{1} n_{0} / 2[\right.} \text { and } \\
\text { for every admissible piece of path } \pi^{\prime} \in \operatorname{AdPath}\left(2 l 2^{2 n_{0}}, c_{1} n_{0} / 2\right): \\
\text { If } \xi \circ \pi=\xi \circ \pi^{\prime}, \text { then } \pi(0) \leq \pi^{\prime}(0) \text { and } \pi\left(c_{1} n_{0} / 2-1\right) \geq \pi^{\prime}\left(c_{1} n_{0} / 2-1\right) .
\end{array}\right\}, \\
& \tilde{E}_{\text {signals II }}^{n_{0}}:=\left\{\begin{array}{l}
\text { For every right ladder path } \pi \in\left(\left[-2 l 2^{2 n_{0}}, 2 l 2^{2 n_{0}}\right] \backslash J_{1}\right)^{\left[0, c_{1} n_{0}[\right.} \text { and } \\
\text { for every admissible piece of path } \pi^{\prime} \in \operatorname{AdPath}\left(2 l 2^{2 n_{0}}, c_{1} n_{0}\right): \\
\text { If } \xi \circ \pi=\xi \circ \pi^{\prime}, \text { then } \pi\left(c_{1} n_{0} / 2\right)=\pi^{\prime}\left(c_{1} n_{0} / 2\right) .
\end{array}\right\} .
\end{aligned}
$$

Note that $\pi^{\prime}$ in the last two definitions may well have some of its values in $J_{1}$. We prove the following modification of Lemma 6.38: 
Lemma 8.29 There exist constants $c_{28}>0, c_{29}>0$ not depending on $n_{0}$ such that:

$$
\tilde{P}\left[\left(\tilde{B}_{\text {signals }}^{n_{0}}\right)^{\mathrm{c}}\right] \leq c_{29} e^{-c_{28} n_{0}} .
$$

Proof. Take a right ladder path

$$
\pi \in\left(\left[-2 l 2^{2 n_{0}}, 2 l 2^{2 n_{0}}\right] \backslash J_{1}\right)^{\left[0, c_{1} n_{0}[\right.}
$$

and an admissible piece of path $\pi^{\prime} \in \operatorname{AdPath}\left(2 \cdot l 2^{2 n}, c_{1} n / 2\right)$ with $\pi(0)>\pi^{\prime}(0)$. We show by induction over $j \in\left[0, c_{1} n / 2\left[\right.\right.$ with the abbreviation $I=\left[0, j+1\left[\right.\right.$ and $I^{\prime}=[0, j[$ :

$$
\tilde{P}\left[\xi \circ \pi \left\lceilI^{\prime}=\xi \circ \pi^{\prime}\left\lceil I^{\prime}\right]=|\mathcal{C}|^{-j} .\right.\right.
$$

Indeed, (8.90) holds for $j=0$. For the induction step $j \mapsto j+1$, we note that $\pi(j)$ is right of all $\pi(i)$ and $\pi^{\prime}(i), i<j$, since $\pi$ is a right ladder path and $\pi(0)>\pi^{\prime}(0)$. Thus $\xi \circ \pi(j)$ is independent of the family $\left(\xi \circ \pi\left\lceil I^{\prime}, \xi \circ \pi^{\prime}\left\lceil I^{\prime}\right)\right.\right.$ with respect to $\tilde{P}$, even if $\pi^{\prime}$ touches the "corrupted" domain $J_{1}$. This is true because $\pi$ does not touch $J_{1}$, and $\xi\left\lceil J_{1}\right.$ is independent of $\xi\left\lceil\left(\mathbb{Z} \backslash J_{1}\right)\right.$ by Lemma 8.1. Therefore, using our induction hypothesis,

$$
\begin{aligned}
& \tilde{P}\left[\xi \circ \pi \left\lceilI=\xi \circ \pi^{\prime}\lceil I]\right.\right. \\
& \quad=\tilde{P}\left[\xi \circ \pi \left\lceilI^{\prime}=\xi \circ \pi^{\prime}\left\lceil I^{\prime}\right] \cdot \tilde{P}\left[\xi \circ \pi(j)=\xi \circ \pi^{\prime}(j)\right]=|\mathcal{C}|^{-j-1} .\right.\right.
\end{aligned}
$$

For $j=c_{1} n_{0} / 2$ we obtain that

$$
\tilde{P}\left[\xi \circ \pi \left\lceil\left[0, c_{1} n_{0} / 2\left[=\xi \circ \pi^{\prime}\left\lceil\left[ 0, c_{1} n_{0} / 2[] \leq|\mathcal{C}|^{-c_{1} n_{0} / 2} .\right.\right.\right.\right.\right.\right.
$$

A similar inductive argument, processing the path $\pi$ in reverse direction, i.e. "from the right to the left", shows that the bound (8.92) also holds when the above condition $\pi(0)>\pi^{\prime}(0)$ is replaced by $\pi\left(c_{1} n_{0} / 2-1\right)<\pi^{\prime}\left(c_{1} n_{0} / 2-1\right)$. There are not more than $4 \cdot l 2^{2 n_{0}}+1 \leq 5 \cdot l 2^{2 n_{0}}$ possible choices for the right ladder path $\pi$, and not more than $5 \cdot l 2^{2 n_{0}}|\mathcal{M}|^{c_{1} n_{0} / 2}$ choices for the admissible path $\pi^{\prime}$. We conclude:

$$
\tilde{P}\left[\left(\tilde{B}_{\text {signals }}^{n_{0}}\right)^{\mathrm{c}}\right] \leq 5 \cdot l 2^{2 n_{0}} \cdot 5 \cdot l 2^{2 n_{0}}|\mathcal{M}|^{c_{1} n_{0} / 2} \cdot|\mathcal{C}|^{-c_{1} n_{0} / 2} .
$$

This implies the claim (8.88), since $|\mathcal{C}|>|\mathcal{M}|$ and $c_{1}$ was chosen large enough; recall Subsection 2.1 .

The following lemma is a variant of Lemma 6.5, which takes care of the "corrupted" domain $J_{1}$.

Lemma 8.30 $\tilde{B}_{\text {signals }}^{n_{0}} \subseteq \tilde{E}_{\text {signals II }}^{n_{0}}$.

Proof of Lemma 8.30. Assume that the event $\tilde{B}_{\text {signals }}^{n_{0}}$ occurs. Let $\pi \in\left(\left[-2 \cdot l 2^{2 n_{0}}, 2 \cdot l 2^{2 n_{0}}\right] \backslash\right.$

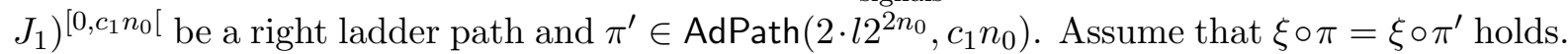
Looking at the first half of $\pi$ and $\pi^{\prime}$ only (with the first points $(0, \pi(0)),\left(0, \pi^{\prime}(0)\right)$ dropped), we see $\pi\left(c_{1} n_{0} / 2\right) \geq \pi^{\prime}\left(c_{1} n_{0} / 2\right)$, since $\tilde{B}_{\text {signals }}^{n_{0}}$ holds. By the same argument, looking at the second half of $\pi$ and $\pi^{\prime}$ only, we infer $\pi\left(c_{1} n_{0} / 2\right) \leq \pi^{\prime}\left(c_{1} n_{0} / 2\right)$. Therefore $\pi\left(c_{1} n_{0} / 2\right)$ and $\pi^{\prime}\left(c_{1} n_{0} / 2\right)$ coincide. This shows that $\tilde{E}_{\text {signals II }}^{n_{0}}$ holds. 


\section{Lemma 8.31}

Assume that the event $B_{\text {all paths, } T^{0}}^{n_{0}} \cap \tilde{B}_{\text {signals }}^{n_{0}} \cap E_{\text {stop }, T^{0}}^{0}$ holds. Let $I \subseteq\left[-6 \cdot 2^{n_{0}}, 6 \cdot 2^{n_{0}}\right] \backslash J_{1}$ be a right ladder interval with $|I|=3 c_{1} n_{0}$, and let $w_{1}, w_{2}, w_{3} \in \mathcal{C}^{c_{1} n_{0}}$ with $\left(\xi\lceil I)_{\rightarrow}=w_{1} w_{2} w_{3}\right.$. Then $\left(w_{1}, w_{2}, w_{3}\right) \in$ Puzzle $_{\mathrm{I}}^{n_{0}}\left(\right.$ Input $\left._{0}\right)$.

This lemma is only a small modification of Lemma 6.7; except of the extra care needed for handling the corrupted domain $J_{1}$, its proof is almost the same.

Proof of Lemma 8.31. Given a right ladder interval $I$ as in the assumption of the lemma, we decompose it into three pieces: Let $I=I_{1} \cup I_{2} \cup I_{3}$, where $I_{1}, I_{2}$, and $I_{3}$ denote the left, middle, and right third of $I$, respectively; thus $\left(\xi\left\lceil I_{i}\right)_{\rightarrow}=w_{i}, i=1,2,3\right.$. Since the event $B_{\text {all paths, } T^{0}}^{n_{0}}$ holds, the straight path which steps through the elements of $I$ from the left to the right in $3 c_{1} n_{0}$ steps is realized at least once by the random walk $(S(t))_{t \geq 0}$ within time $2^{2 n_{0}}$ of a stopping time $T_{k}^{0}, k<2^{\alpha n_{0}}$. Observing $\xi$ along such a straight path generates the word $w_{1} w_{2} w_{3}$. Thus

$$
\left(w_{1}, w_{2}, w_{3}\right) \in \text { PrePuzzle }^{n_{0}} \text { (Input). }
$$

Let $w_{2}^{\prime}$ be such that $\left(w_{1}, w_{2}^{\prime}, w_{3}\right) \in$ PrePuzzle ${ }^{n_{0}}$ (Input). In order to prove the claim $\left(w_{1}, w_{2}, w_{3}\right) \in$ Puzzle $\mathrm{I}_{\mathrm{I}}^{n_{0}}$ (Input) it remains to show: $w_{2}=w_{2}^{\prime}$. When the event $E_{\text {stop }, T^{0}}^{0}$ holds, the stopping times of $T_{k}^{0}, k<2^{\alpha n_{0}}$, all stop the random walk $(S(t))_{t \geq 0}$ somewhere in the interval $\left[-2^{n_{0}}, 2^{n_{0}}\right]$. Within time $2^{2 n_{0}}$ the random walk moves at most a distance $l 2^{2 n_{0}}$. Because of $w_{1} w_{2}^{\prime} w_{3} \in$ PrePuzzle ${ }^{n_{0}}$ (Input), the word $w_{1} w_{2}^{\prime} w_{3}$ occurs somewhere in the observations at most $2^{2 n_{0}}$ time steps after a stopping time $T_{k}^{0}, k<2^{\alpha n_{0}}$. Within time $2^{2 n_{0}}$ after a stopping time, the random walk cannot be further away from the origin than $l 2^{2 n_{0}}+2^{n_{0}} \leq 2 \cdot l 2^{2 n_{0}}$, since the event $E_{\text {stop, } T^{0}}^{0}$ holds. Thus there exists an admissible piece of path $R^{\prime}:\left[0,3 c_{1} n_{0}\left[\rightarrow\left[-2 \cdot l 2^{2 n_{0}}, 2 \cdot l 2^{2 n_{0}}\right]\right.\right.$ such that $\xi \circ R^{\prime}=w_{1} w_{2}^{\prime} w_{3}$. Let $R:\left[0,3 c_{1} n_{0}\left[\rightarrow I \subseteq\left[-2 \cdot l 2^{2 n_{0}}, 2 \cdot l 2^{2 n_{0}}\right]\right.\right.$ denote the right ladder path which passes through $I$ from the left to the right. Note that $R$ does not meet the "corrupted" domain $J_{1}$, although we need not assume that $R^{\prime}$ does not meet $J_{1}$. We know $\xi \circ R^{\prime}\left\lceil\left[0, c_{1} n_{0}\right.\right.$ [= $\xi \circ R\left\lceil\left[0, c_{1} n_{0}\left[=w_{1}\right.\right.\right.$ and $\left(\xi \circ R^{\prime}\left\lceil\left[2 c_{1} n_{0}, 3 c_{1} n_{0}[)_{\rightarrow}=\left(\xi \circ R\left\lceil\left[2 c_{1} n_{0}, 3 c_{1} n_{0}[) \rightarrow=w_{3}\right.\right.\right.\right.\right.\right.$. Furthermore, the event $\tilde{E}_{\text {signals II }}^{n_{0}} \supseteq \tilde{B}_{\text {signals }}^{n_{0}}$ holds; see Lemma 8.30. Abbreviating $x:=c_{1} n_{0} / 2$ and $y:=5 c_{1} n_{0} / 2$, this implies $R^{\prime}(x)=R(x)$ and $R^{\prime}(y)=R(y)$. But $R\left\lceil[x, y]\right.$ is a right ladder path; thus $R^{\prime}\lceil[x, y]$ must be the same right ladder path, since only right ladder paths can travel equally fast to the right as $R$ does. Hence $w_{2}=\left(\xi \circ R\left\lceil\left[c_{1} n_{0}, 2 c_{1} n_{0}[) \rightarrow=\left(\xi \circ R^{\prime}\left\lceil\left[c_{1} n_{0}, 2 c_{1} n_{0}[) \rightarrow=w_{2}^{\prime}\right.\right.\right.\right.\right.\right.$. This finishes the proof of Lemma 8.31.

Next we show that under appropriate conditions Center ${ }_{I I I}$ is indeed a lower bound for Puzzle $\mathrm{IV}_{\mathrm{IV}}(\chi)$.

Lemma 8.32 If the event $B_{\text {all paths, } T^{0}}^{n_{0}} \cap B_{\text {all paths II }}^{n_{0}} \cap \tilde{B}_{\text {signals }}^{n_{0}} \cap E_{\text {stop }, T^{0}}^{0}$ holds, then Center ${ }_{\text {III }} \subseteq$ Puzzle $n_{\text {IV }}^{n_{0}}(\chi)$.

Proof. Assume that $B_{\text {all paths }, T^{0}}^{n_{0}} \cap B_{\text {all paths II }}^{n_{0}} \cap \tilde{B}_{\text {signals }}^{n_{0}} \cap E_{\text {stop }, T^{0}}^{0}$ holds, and let $w_{2} \in$ Center III $_{\text {. }}$ Then $w_{2}=\left(\xi\lceil I)_{\rightarrow}\right.$ for some right ladder interval $I \subseteq\left[-2 \cdot 2^{n_{0} / 2}, 2 \cdot 2^{n_{0} / 2}\right] \backslash\left[-2^{n_{0} / 2}, 2^{n_{0} / 2}\right]$, $|I|=c_{1} n_{0}$. We take the larger right ladder interval $I^{\prime} \supseteq I,\left|I^{\prime}\right|=3 c_{1} n_{0}$, with $c_{1} n_{0}$ extra points to the left of $I$ and another $c_{1} n_{0}$ extra points to the right of $I$; then $I^{\prime} \subseteq\left[-3 \cdot 2^{n_{0} / 2}, 3 \cdot 2^{n_{0} / 2}\right] \backslash J_{1} \subseteq$ $\left[-6 \cdot 2^{n_{0}}, 6 \cdot 2^{n_{0}}\right] \backslash J_{1} ;$ note that $\operatorname{dist}\left(J_{1}, \mathbb{Z} \backslash\left[-2^{n_{0} / 2}, 2^{n_{0} / 2}\right]\right)>c_{1} n_{0} l$ and $\operatorname{dist}\left(\left[-2 \cdot 2^{n_{0} / 2}, 2 \cdot 2^{n_{0} / 2}\right], \mathbb{Z} \backslash\right.$ $\left.\left[-3 \cdot 2^{n_{0} / 2}, 3 \cdot 2^{n_{0} / 2}\right]\right)>c_{1} n_{0} l$; recall that $n_{0}$ is chosen large enough (Subsection 2.1). Then $\left(\xi\left\lceil I^{\prime}\right)_{\rightarrow}=w_{1} w_{2} w_{3}\right.$ for some $w_{1}, w_{3} \in \mathcal{C}^{c_{1} n_{0}}$. Using that the events $B_{\text {all paths, } T^{0}}^{n_{0}}, \tilde{B}_{\text {signals }}^{n_{0}}$, and $E_{\text {stop }, T^{0}}^{0}$ hold, Lemma 8.31 implies $\left(w_{1}, w_{2}, w_{3}\right) \in$ Puzzle $_{\mathrm{I}}^{n_{0}}\left(\right.$ Input $\left._{0}\right)$. Let $R$ denote the (unique) right ladder path $R:\left[0,3 c_{1} n_{0}\left[\rightarrow I^{\prime}\right.\right.$. Since $B_{\text {all paths II }}^{n_{0}}$ holds, the random walk $S$ follows $R$ 
(time-shifted) at most $2^{n_{0}} / l$ time steps after some stopping time $T_{k}^{0}(\chi), k \in\left[0,2^{\alpha n_{0}}[\right.$. Then $\xi \circ R=w_{1} w_{2} w_{3}$; thus $\left(w_{1}, w_{2}, w_{3}\right) \in$ Puzzle $_{\mathrm{III}}^{n_{0}}(\chi)$ by Definition $(8.42)$; hence $w_{2} \in$ Puzzle $\mathrm{I}_{\mathrm{IV}}^{n_{0}}(\chi)$ by Definition (8.43). This proves the lemma.

Definition 8.33 We set

$$
\begin{aligned}
& E_{\text {center }}^{1}:=\left\{\mid \text { Center }_{\text {III }} \cap \text { Puzzle }_{\mathrm{IV}}^{n_{0}}(\chi) \mid \geq 2^{n_{0} / 3} \text { and } \mid \text { Puzzle }_{\mathrm{IV}}^{n_{0}}(\chi) \mid \leq 50 \cdot 2^{n_{0}}\right\} \\
& \Xi_{\text {center }}^{1}:=\left\{\xi \in \mathcal{C}^{\mathbb{Z}} \mid P\left[E_{\text {center }}^{1} \mid \xi\right] \geq \frac{1}{2}\right\} .
\end{aligned}
$$

The sets $E_{\text {center }}^{1}$ and $\Xi_{\text {center }}^{1}$ play an analogous role for the stopping times $T^{1}$ as $E_{\text {reconst, } f}^{m}$ and $\Xi_{\text {reconst,f }}^{m}$ play for the "higher level" stopping times in Section 7.

Lemma 8.34 For some positive constants $c_{53}$ and $c_{54}$ holds $\tilde{P}\left[\xi \in \Xi_{\text {center }}^{1}\right] \geq 1-c_{53} e^{-c_{54} n_{0}}$.

Proof. We claim first that

$$
B_{\text {all paths, } T^{0}}^{n_{0}} \cap B_{\text {all paths II }}^{n_{0}} \cap \tilde{B}_{\text {signals }}^{n_{0}} \cap E_{\text {stop }, T^{0}}^{0} \cap B_{\text {recogn straight }}^{n_{0}}\left(J_{1}\right) \cap B_{\text {unique fit }}^{n_{0}}\left(J_{1}\right) \subseteq E_{\text {center }}^{1}
$$

holds. To prove this, assume that the intersection of events on the left hand side in (8.97) occurs. Since the $B_{\text {all paths, } T^{0}}^{n_{0}}, B_{\text {all paths II }}^{n_{0}} \tilde{B}_{\text {signals }}^{n_{0}}$, and $E_{\text {stop, } T^{0}}^{0}$ occur, Lemma 8.32 implies |Center $\mathrm{III} \cap$ Puzzle $e_{\text {IV }}^{n_{0}}(\chi)|=|$ Center $_{I I I} \mid$. Using that the event $B_{\text {unique fit }}^{n_{0}}\left(J_{1}\right)$, Lemma 8.18 yields $\mid$ Center III $\mid \geq$

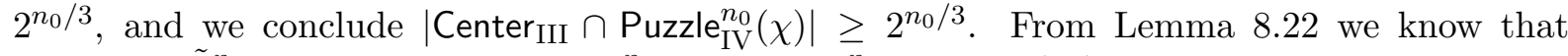
the event $\tilde{E}_{\text {only ladder }}^{n_{0}}$ occurs, since $B_{\text {all paths, } T^{0}}^{n_{0}} \cap B_{\text {recogn straight }}^{n_{0}}\left(J_{1}\right)$ occurs. Using this and the occurrence of the event $E_{\text {stop }, T^{0}}^{0}$, Lemma 8.23 implies $\left|\operatorname{Puzzle}_{\mathrm{IV}}^{n_{0}}(\chi)\right| \leq \mid$ Center $_{\mathrm{II}} \mid$, and finally Lemma 8.18 yields $\mid$ Center $_{\text {II }} \mid \leq 50 \cdot 2^{n_{0}}$. Summarizing, we know $\left|\operatorname{Puzzle}_{\mathrm{IV}}^{n_{0}}(\chi)\right| \leq 50 \cdot 2^{n_{0}}$. Thus we have shown that the event $E_{\text {center }}^{1}$ occurs; this proves the claim (8.97). Taking probabilities and using $\tilde{E}_{\text {stop }, T^{0}}^{0} \subseteq E_{\text {stop }, T^{0}}^{0}$, we get

$$
\begin{aligned}
\tilde{P} & {\left[E_{\text {center }}^{1}\right] } \\
\geq & \tilde{P}\left[B_{\text {all paths, } T^{0}}^{n_{0}} \cap B_{\text {all paths II }}^{n_{0}} \cap \tilde{B}_{\text {signals }}^{n_{0}} \cap E_{\text {stop, } T^{0}}^{0} \cap B_{\text {recogn straight }}^{n_{0}}\left(J_{1}\right) \cap B_{\text {unique fit }}^{n_{0}}\left(J_{1}\right)\right] \\
\geq & \tilde{P}\left[\tilde{E}_{\text {stop }, T^{0}}^{0}\right]-\tilde{P}\left[\left(B_{\text {all paths }, T^{0}}^{n_{0}}\right)^{\mathrm{c}} \cap E_{\text {stop }, T^{0}}^{0}\right]-\tilde{P}\left[\left(B_{\text {all paths II }}^{n_{0}} \cap \tilde{E}_{\text {stop }, T^{0}}^{0}\right]\right. \\
& -\tilde{P}\left[\left(\tilde{B}_{\text {recogn straight }}^{n_{0}}\left(J_{1}\right)\right)^{\mathrm{c}}\right]-\tilde{P}\left[\left(\tilde{B}_{\text {signals }}^{n_{0}}\right)^{\mathrm{c}}\right]-\tilde{P}\left[\left(B_{\text {unique fit }}^{n_{0}}\left(J_{1}\right)\right)^{\mathrm{c}}\right] \\
\geq & 1-c_{55} e^{-c_{54} n_{0}}
\end{aligned}
$$

for some positive constants $c_{55}$ and $c_{54}$ by Theorem 8.5 and Lemmas 8.24, 8.27, 8.20, 8.29, and 8.17. Hence we obtain the following:

$$
\frac{1}{2} \tilde{P}\left[\xi \notin \Xi_{\text {center }}^{1}\right]=\frac{1}{2} \tilde{P}\left[P\left[\left(E_{\text {center }}^{1}\right)^{\mathrm{c}} \mid \xi\right]>\frac{1}{2}\right] \leq \tilde{P}\left[\left(E_{\text {center }}^{1}\right)^{\mathrm{c}}\right] \leq c_{55} e^{-c_{54} n_{0}}
$$

recall that $\tilde{P}[\cdot \mid \xi]$ and $P[\cdot \mid \xi]$ coincide.

The following definition is yet another modification Definition 7.3. 
Definition 8.35 Let $v(k)$ denote again the $(k+1)$ st visit of $S$ to the origin. We define

$$
\begin{aligned}
& \mathbb{T}^{1 \prime}(\xi, \chi):=\left\{t \in \mathbb{N} \mid \begin{array}{l}
\mid \text { Center III } \cap \text { Puzzle }_{\text {IV }}^{n_{0}}\left(\theta^{t} \chi\right) \mid \geq 2^{n_{0} / 3} \\
\text { and } \mid \text { Puzzle } n_{\mathrm{IV}}^{n_{0}}\left(\theta^{t} \chi\right) \mid \leq 50 \cdot 2^{n_{0}}
\end{array}\right\}, \\
& E_{\text {when back recog }}^{1}:=\left\{\begin{array}{l}
\text { For more than } 1 / 4 \text { of the points } k \in\left[0,2^{2 \alpha n_{1}}[\right. \\
\text { holds } v\left(k 2^{\alpha n_{1}}\right) \in \mathbb{T}^{1 \prime}(\xi, \chi)
\end{array}\right\} .
\end{aligned}
$$

Here is yet another analogue to Lemma 7.4:

Lemma 8.36 If the event $B_{\text {all paths }, T^{0}}^{n_{0}} \cap B_{\text {all paths II }}^{n_{0}} \cap \tilde{B}_{\text {signals }}^{n_{0}} \cap E_{\text {stop }, T^{0}}^{0}$ holds, then $\mathbb{T}^{1}(\chi) \supseteq$ $\mathbb{T}^{1 \prime}(\xi, \chi) \cap\left[0,2^{12 \alpha n_{1}}-2 \cdot 2^{12 \alpha n_{0}}[\right.$.

Proof. Assuming that the event $B_{\text {all paths }, T^{0}}^{n_{0}} \cap B_{\text {all paths II }}^{n_{0}} \cap \tilde{B}_{\text {signals }}^{n_{0}} \cap E_{\text {stop, } T^{0}}^{0}$ holds, we know Center $_{\text {III }} \subseteq$ Puzzle $_{\text {IV }}^{n_{0}}(\chi)$ by Lemma 8.32; thus $\mid$ Puzzle $_{\text {IV }}^{n_{0}}(\chi) \cap$ Puzzle $_{\text {IV }}^{n_{0}}\left(\theta^{t} \chi\right)|\geq|$ Center $_{\text {III }} \cap$ Puzzle $\mathrm{IV}_{\mathrm{IV}}^{n_{0}}\left(\theta^{t} \chi\right) \mid$ for all $t$. This implies the claim $\mathbb{T}^{1}(\chi) \supseteq \mathbb{T}^{1 \prime}(\xi, \chi) \cap\left[0,2^{12 \alpha n_{1}}-2 \cdot 2^{12 \alpha n_{0}}\right.$ [ of the lemma; recall Definition (8.44) of $\mathbb{T}^{1}(\chi)$.

The following lemma is another variant of Lemma 7.5 ; only the stopping times $\mathbb{T}_{f}$ and $\mathbb{T}_{f}^{\prime}$ are replaced by $\mathbb{T}^{1}$ and $\mathbb{T}^{1 \prime}$, respectively, and $T_{f}$ is replaced by $T^{1}$.

Lemma 8.37 Assume that the events

$E_{\text {no error, } T^{1}}^{1} \cap E_{\text {enough back }}^{1} \cap E_{\text {when back recog }}^{1}$ and $\mathbb{T}^{1}(\chi) \supseteq \mathbb{T}^{1 \prime}(\xi, \chi) \cap\left[0,2^{12 \alpha n_{1}}-2 \cdot 2^{12 \alpha n_{0}}[\right.$ hold. Then $E_{\text {stop }, T^{1}}^{1}$ holds, too.

Proof. Using that the event $E_{\text {enough back }}^{1}$ holds, we know

$$
v\left(k 2^{\alpha n_{1}}\right) \in\left[0,2^{12 \alpha n_{1}} / 8\right] \subseteq\left[0,2^{12 \alpha n_{1}}-2 \cdot 2^{12 \alpha n_{0}}[\right.
$$

for all $k \in\left[0,2^{2 \alpha n_{1}}\left[\right.\right.$; recall the definition (7.2) of the event $E_{\text {enough back }}^{1}$, and recall that $v\left(k 2^{\alpha n_{1}}\right)$ denotes the $k 2^{\alpha n_{1}}$-st return time of the random walk $S$ to the origin.

Since the event $E_{\text {when back recog }}^{1}$ holds, we obtain $\left|\mathbb{T}^{1}(\chi)\right| \geq \mid \mathbb{T}^{1 \prime}(\xi, \chi) \cap\left[0,2^{12 \alpha n_{1}}-2 \cdot 2^{12 \alpha n_{0}}[\mid \geq\right.$ $2^{2 \alpha n_{1}} / 4$. By Definition (8.45) of the stopping times $T^{1}$, this yields $T_{k}^{1}(\chi)<2^{12 \alpha n_{1}}$ for all $k<\left(2^{2 \alpha n_{1}} / 4\right) /\left(2 \cdot 2^{2 n_{1}}\right)=2^{2(\alpha-1) n_{1}} / 8$. The event $E_{\text {no error }, T^{1}}^{1}$ holds, and $2^{2(\alpha-1) n_{1}} / 8 \geq 2^{\alpha n_{1}}$; recall that $\alpha$ and $n_{1} \geq n_{0}$ are large (see Section 2.1). Hence we obtain $\left|S\left(T_{k}^{1}(\chi)\right)\right| \leq 2^{n_{1}}$ for all $k \in\left[0,2^{\alpha n_{1}}\left[\right.\right.$; recall the definition (7.1) of the event $E_{\text {no error, } T^{1}}^{1}$. Using Definition (8.45) again, we see that $T_{j}^{1}(\chi)+2 \cdot 2^{2 n_{1}} \leq T_{k}^{1}(\chi)$ is automatically fulfilled for $j<k$ whenever $T_{k}^{1}(\chi)<2^{12 \alpha n_{1}}$, which is the case at least for $k \in\left[0,2^{\alpha n_{1}}\right.$ [. Summarizing, we have proven that the event $E_{\text {stop, } T^{1}}^{1}$ holds; recall its definition (3.14).

The following Lemma is another modification of Lemma 7.6.

Lemma 8.38 We have the bound

$$
\tilde{P}\left[\left(E_{\text {when back recog }}^{1}\right)^{\mathrm{c}} \cap\left\{\xi \in \Xi_{\text {center }}^{1}\right\}\right] \leq 0.9^{2^{2 \alpha n_{1}}} .
$$

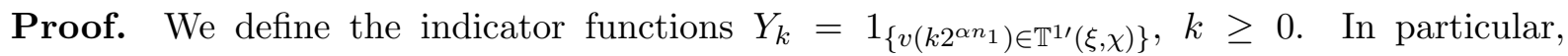
$E_{\text {when back recog }}^{1}=\left\{2^{-2 \alpha n_{1}} \sum_{k=0}^{2^{2 \alpha n_{1}}-1} Y_{k}>1 / 4\right\}$ holds. Note that $v\left((k+1) 2^{\alpha n_{1}}\right)-v\left(k 2^{\alpha n_{1}}\right) \geq$ $2^{\alpha n_{1}}>2 \cdot 2^{12 \alpha n_{0}}$. Recall that $P$ and $\tilde{P}$ differ only in the distribution of the scenery $\xi$, but not in the law of the random walk $S$. In particular, $P[\cdot \mid \xi]=\tilde{P}[\cdot \mid \xi]$ holds. By the strong Markov 
property of the random walk $(S(k))_{k \geq 0}$, conditioned under $\xi$, the variables $\left(Y_{k}\right)_{k \geq 0}$ are i.i.d. Bernoulli variables. Furthermore, we have $E\left[Y_{k} \mid \xi\right]=P\left[E_{\text {center }}^{1} \mid \xi\right] \geq 1 / 2$ whenever $\xi \in \Xi_{\text {center }}^{1}$. Just as in (7.10), the exponential Chebyshev inequality implies for these sceneries $\xi$ :

$$
\tilde{P}\left[\left(E_{\text {when back recog }}^{1}\right)^{\mathrm{c}} \mid \xi\right] \leq\left(\frac{e^{1 / 4}+e^{-3 / 4}}{2}\right)^{2^{2 \alpha n_{1}}} \leq 0.9^{2^{2 \alpha n_{1}}} .
$$

Taking the expectation with respect to $\tilde{P}$, this implies the claim (8.103), just as in (7.11).

Proof of Theorem 3.8. Recall that $\tilde{E}_{\mathrm{stop}, T^{0}}^{0} \subseteq E_{\mathrm{stop}, T^{0}}^{0}$. Using this and Lemmas 8.36 and 8.37, we get

$E_{\text {no error }, T^{1}}^{1} \cap E_{\text {enough back }}^{1} \cap E_{\text {when back recog }}^{1} \cap B_{\text {all paths }, T^{0}}^{n_{0}} \cap B_{\text {all paths II }}^{n_{0}} \cap \tilde{B}_{\text {signals }}^{n_{0}} \cap \tilde{E}_{\text {stop }, T^{0}}^{0} \subseteq E_{\text {stop }, T^{1}}^{1}$.

Since $E_{\text {enough back }}^{1}$ depends only on $S$ but not on $\xi$, we have $\tilde{P}\left[\left(E_{\text {enough back }}^{1}\right)^{\mathrm{c}}\right]=P\left[\left(E_{\text {enough back }}^{1}\right)^{\mathrm{c}}\right]$. Thus, using Lemmas 8.25, 7.2, 8.38, 8.34, 8.24, 8.27, 8.29, and Theorem 8.5, we know

$$
\begin{aligned}
\tilde{P} & {\left[\left(E_{\text {stop }, T^{1}}^{1}\right)^{\mathrm{c}}\right] } \\
\leq & \tilde{P}\left[\left(E_{\text {no error }, T^{1}}^{1}\right)^{\mathrm{c}}\right]+\tilde{P}\left[\left(E_{\text {enough back }}^{1}\right)^{\mathrm{c}}\right]+\tilde{P}\left[\left(E_{\text {when back recog }}^{1}\right)^{\mathrm{c}} \cap\left\{\xi \in \Xi_{\text {center }}^{1}\right\}\right] \\
& +\tilde{P}\left[\xi \notin \Xi_{\text {center }}^{1}\right]+\tilde{P}\left[\left(B_{\text {all paths }, T^{0}}^{n_{0}}\right)^{\mathrm{c}} \cap E_{\text {stop }, T^{0}}^{0}\right]+\tilde{P}\left[\left(B_{\text {all paths II }}^{n_{0}} \cap \tilde{E}_{\text {stop }, T^{0}}^{0}\right]\right. \\
& +\tilde{P}\left[\left(\tilde{B}_{\text {signals }}^{n_{0}}\right)^{\mathrm{c}}\right]+\tilde{P}\left[\left(\tilde{E}_{\text {stop }}^{0}\right)^{\mathrm{c}}\right] \\
\leq & c_{48} e^{-c_{49} n_{0}}+c_{35} 2^{-\alpha n_{1}}+0.9^{2^{2 \alpha n_{1}}}+c_{53} e^{-c_{54} n_{0}}+c_{21} e^{-c_{20} n_{0}} \\
& +c_{50} e^{-c_{51} n_{0}}+c_{29} e^{-c_{28} n_{0}}+c_{36} e^{-c_{37} n_{0}} \\
\leq & e^{-c_{4} n_{0}}
\end{aligned}
$$

since $n_{0}$ is chosen large enough (see Subsection 2.1)

Acknowledgment. We thank an anonymous referee for a very careful reading of an earlier version of this manuscript.

\section{References}

[1] Itai Benjamini and Harry Kesten. Distinguishing sceneries by observing the scenery along a random walk path. J. Anal. Math., 69:97-135, 1996.

[2] Krzysztof Burdzy. Some path properties of iterated Brownian motion. In Seminar on Stochastic Processes, 1992 (Seattle, WA, 1992), pages 67-87. Birkhäuser Boston, Boston, MA, 1993.

[3] Frank den Hollander and Jeffrey E. Steif. Mixing properties of the generalized $T, T^{-1}$ process. J. Anal. Math., 72:165-202, 1997.

[4] W. Th. F. den Hollander. Mixing properties for random walk in random scenery. Ann. Probab., 16(4):1788-1802, 1988. 
[5] Richard Durrett. Probability: theory and examples. Duxbury Press, Belmont, CA, second edition, 1996.

[6] C. Douglas Howard. Detecting defects in periodic scenery by random walks on $\mathbb{Z}$. Random Structures Algorithms, 8(1):59-74, 1996.

[7] C. Douglas Howard. Orthogonality of measures induced by random walks with scenery. Combin. Probab. Comput., 5(3):247-256, 1996.

[8] C. Douglas Howard. Distinguishing certain random sceneries on $\mathbb{Z}$ via random walks. Statist. Probab. Lett., 34(2):123-132, 1997.

[9] Steven Arthur Kalikow. T, $T^{-1}$ transformation is not loosely Bernoulli. Ann. of Math. (2), 115(2):393-409, 1982.

[10] M. Keane and W. Th. F. den Hollander. Ergodic properties of color records. Phys. A, 138(1-2):183-193, 1986.

[11] Harry Kesten. Detecting a single defect in a scenery by observing the scenery along a random walk path. In Itô's stochastic calculus and probability theory, pages 171-183. Springer, Tokyo, 1996.

[12] Harry Kesten. Distinguishing and reconstructing sceneries from observations along random walk paths. In Microsurveys in discrete probability (Princeton, NJ, 1997), pages 75-83. Amer. Math. Soc., Providence, RI, 1998.

[13] A. Lenstra and H. Matzinger. Reconstructing a 4-color scenery by observing it along a recurrent random walk path with unbounded jumps. In preparation, 2004.

[14] Elon Lindenstrauss. Indistinguishable sceneries. Random Structures Algorithms, 14(1):7186, 1999 .

[15] Matthias Löwe and Heinrich Matzinger, III. Scenery reconstruction in two dimensions with many colors. Ann. Appl. Probab., 12(4):1322-1347, 2002.

[16] Matthias Löwe and Heinrich Matzinger, III. Reconstruction of sceneries with correlated colors. Stochastic Process. Appl., 105(2):175-210, 2003.

[17] Heinrich Matzinger. Reconstructing a 2-color scenery by observing it along a simple random walk path. To appear in The Annals of Applied Probability.

[18] Heinrich Matzinger. Reconstructing a 2-color scenery by observing it along a simple random walk path with holding. PhD-thesis, Cornell University, 1999.

[19] Heinrich Matzinger. Reconstructing a three-color scenery by observing it along a simple random walk path. Random Structures Algorithms, 15(2):196-207, 1999.

[20] Heinrich Matzinger and Silke W. W. Rolles. Finding blocks and other patterns in a random coloring of $\mathbb{Z}$. Preprint, 2003.

[21] Heinrich Matzinger and Silke W. W. Rolles. Reconstructing a piece of scenery with polynomially many observations. Stochastic Process. Appl., 107(2):289-300, 2003. 
[22] Heinrich Matzinger and Silke W. W. Rolles. Reconstructing a random scenery observed with random errors along a random walk path. Probab. Theory Related Fields, 125(4):539-577, 2003.

[23] Heinrich Matzinger and Silke W. W. Rolles. Retrieving random media. Preprint, 2003.

[24] Frank Spitzer. Principles of random walks. Springer-Verlag, New York, second edition, 1976. Graduate Texts in Mathematics, Vol. 34. 